EBERHARD WILLE

MANFRED ALBRING (Hrsg.)

\title{
REFORMOPTIONEN IM GESUNDHEITSWESEN
}


EBERHARD WILLE

MANFRED ALBRING (Hrsg.)

\section{REFORMOPTIONEN IM GESUNDHEITSWESEN}

Der Sammelband enthält Referate, Berichte aus Arbeitsgruppen und Diskussionsbeiträge eines interdisziplinären Workshops über Reformoptionen im Gesundheitswesen, insbesondere im Bereich der gesetzlichen Krankenversicherung (GKV). Das Themenspektrum umfaßt alternative Finanzierungsmöglichkeiten, Strukturverträge und Modellvorhaben und den Leistungskatalog der GKV im Lichte des Solidaritätsprinzips. Der Teilnehmerkreis setzte sich aus Vertretern der Ärzteschaft, der Krankenkassen und -versicherungen, der pharmazeutischen Industrie, der Wissenschaft, der Ministerialbürokratie und der Politik zusammen.

Eberhard Wille wurde 1942 in Berlin geboren. Nach dem Dipl.-Examen 1966 an der Universität Bonn, der Promotion 1969 und der Habilitation 1973 an der Universität Mainz, ist er seit 1975 Professor für Volkswirtschaftslehre und Finanzwissenschaft an der Universität Mannheim. Er ist u.a. Mitglied des Wissenschaftlichen Beirats beim Bundesministerium für Wirtschaft und des Sachverständigenrates für die Konzertierte Aktion im Gesundheitswesen.

Manfred Albring wurde 1943 in Bochum geboren. Studium der Humanmedizin an der Universität Marburg. Bei der Schering AG ist er Leiter Medizin und Gesundheitswesen des Geschäftsbereichs Deutschland. Er ist u.a. Mitglied der Arbeitsgemeinschaft für dermatologische Forschung, der Deutschen Pharmakologischen Gesellschaft und des Kuratoriums der Deutschen Herzstiftung. 
Reformoptionen im Gesundheitswesen

Eberhard Wille and Manfred Albring - 978-3-631-75598-3

Downloaded from PubFactory at 01/11/2019 03:14:58AM

via free access 


\section{ALLOKATION IM \\ MARKTWIRTSCHAFTLICHEN SYSTEM}

Herausgegeben von

Heinz König, Hans-Heinrich Nachtkamp,

Ulrich Schlieper, Eberhard Wille

Band 41

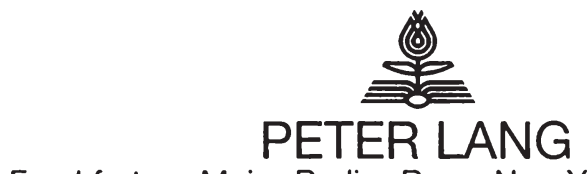

Frankfurt am Main - Berlin - Bern - New York - Paris - Wien 


\section{EBERHARD WILLE MANFRED ALBRING \\ (Hrsg.)}

\section{REFORMOPTIONEN IM GESUNDHEITSWESEN}

Bad Orber Gespräche über kontroverse Themen im Gesundheitswesen 7.-8.11.1997

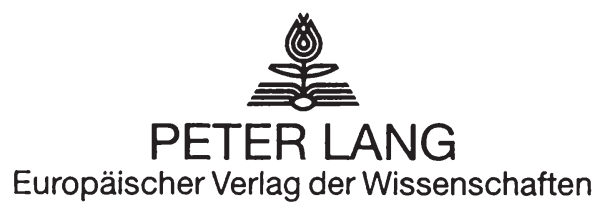


Die Deutsche Bibliothek - CIP-Einheitsaufnahme

Reformoptionen im Gesundheitswesen / Bad Orber Gespräche über kontroverse Themen im Gesundheitswesen 7.-8.11.1997 / Eberhard Wille / Manfred Albring (Hrsg.). - Frankfurt am Main ; Berlin ; Bern ; New York ; Paris ; Wien : Lang, 1998 (Allokation im marktwirtschaftlichen System ; Bd. 41) ISBN 3-631-33563-6

Open Access: The online version of this publication is published on www.peterlang.com and www.econstor.eu under the international Creative Commons License CC-BY 4.0. Learn more on how you can use and share this work: http://creativecommons. org/licenses/by/4.0.

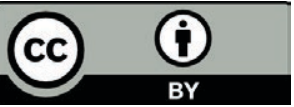

This book is available Open Access thanks to the kind support of ZBW - Leibniz-Informationszentrum Wirtschaft.

Gedruckt auf alterungsbeständigem, säurefreiem Papier.

ISBN 3-631-33563-6

ISBN 978-3-631-75385-9 (eBook)

(C) Peter Lang $\mathrm{GmbH}$

Europäischer Verlag der Wissenschaften

Frankfurt am Main 1998

Alle Rechte vorbehalten.

Das Werk einschließlich aller seiner Teile ist urheberrechtlich geschützt. Jede Verwertung außerhalb der engen Grenzen des Urheberrechtsgesetzes ist ohne Zustimmung des Verlages unzulässig und strafbar. Das gilt insbesondere für Vervielfältigungen, Übersetzungen, Mikroverfilmungen und die Einspeicherung und Verarbeitung in elektronischen Systemen.

Printed in Germany 124567 


\section{Danksagung}

Die Planung, Vorbereitung und Durchführung der Bad Orber Gespräche ist mit einem erheblichen Arbeitsaufwand verbunden. Bis zur Fertigstellung des Buches haben alle Beteiligten außerordentlich viel Engagement und Zähigkeit bewiesen. Stellvertretend für die vielen Beteiligten sei hier noch einmal ausdrücklich Ingrid Czemper, Martina Kuchenbecker, Hans-Michael Kreht und Markus Schröder gedankt. 
Eberhard Wille and Manfred Albring - 978-3-631-75598-3

Downloaded from PubFactory at 01/11/2019 03:14:58AM

via free access 


\section{Inhaltsverzeichnis}

Helmut Laschet

Manfred Albring

Eberhard Wille

Michael Arnold

Herbert Rebscher

Eva Walzik

Anita B. Pfaff

Frank E. Münnich

Gerd Glaeske

Lothar Krimmel

Wolfgang Schmeinck

Alexander P. F. Ehlers

Herwig F. Schirmer

Dominik Graf von Stillfried

Dieter Cassel

Gerhard Schulte

Hans-Jürgen Firnkorn

Eberhard Wille

Verzeichnis der Teilnehmer
Vorwort

Zwischen Endzeitstimmung und Aufbruch

- der Wettbewerb als Suchprozeß

Begrüßung

8

12

Aktuelle Anlässe und Ansatzpunkte der

sen

Entwicklungsperspektiven der medizinischen Versorgung

Finanzierungsoptionen aus Sicht der Ersatzkassen

Finanzierungsoptionen der gesetzlichen Krankenversicherung

Einnahmenentwicklung der GKV und Finanzierungsoptionen

Strukturverträge und Modellvorhaben neue Tätigkeitsfelder für die GKV

Strukturverträge und Modellvorhaben aus kassenärztlicher Sicht

Chancen und Risiken von Strukturverträgen und Modellvorhaben

Der Leistungskatalog der Krankenkassen im Lichte des Solidaritätsprinzips

Anmerkungen zum Solidaritätsprinzip

Der Leistungskatalog der GKV im Lichte des Solidarprinzips

Neuordnung des Leistungskataloges der GKV als Reformoption?

Einnahmenentwicklung der GKV und Finanzierungsoptionen: Zusammenfassung Arbeitsgruppe 1

Chancen und Risiken von Strukturverträgen und Modellvorhaben: Zusammenfassung Arbeitsgruppe 2 daritätsprinzips: Zusammenfassung Arbeitsgruppe 3

Resümee: Stabilisierung der gesetzlichen Krankenversicherung mit Hilfe adaptiver Reformen 40

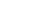
2 9 5 4 


\section{Vorwort \\ Zwischen Endzeitstimmung und Aufbruch - der Wett- bewerb als Suchprozeß}

Helmut Laschet

Die letzten Jahre eines Jahrhunderts sind erfahrungsgemäß durch dunkle Endzeitstimmungen geprägt. Das war kennzeichnend für das ausgehende 19. Jahrhundert, und dies scheint sich ähnlich auch in unserer Zeit zu wiederholen.

Nach 50 Jahren fast linearen Wohlstandswachstums wächst in den reifen Industrie- und Dienstleistungsgesellschaften des Westens die Ahnung, daß sich die vergangene Entwicklung nicht ins nächste Jahrhundert extrapolieren läßt. Scheinbar unumstößlich geltende Wertesysteme geraten ins Wanken, und der Abschied davon ist wohl vor allem deshalb schmerzhaft, weil die bislang geltende Werteordnung zugleich auch das Gefühl von Sicherheit und Wohlstand vermittelt hat, die nun in Frage steht.

Der vorliegende Dokumentationsband eines zweitägigen Symposions am 7. und 8. November 1997 zeigt in mehreren Dimensionen die Herausforderungen, vor denen die Medizin und ihre Einbettung in das System der sozialen Sicherung steht.

Quasi als Advocatus Diaboli haben der Mediziner Professor Michael Arnold und der Ökonom Professor Frank Münnich in die Leistungsfähigkeit der Medizin für die gesundheitliche Versorgung und in die Belastungsfähigkeit der Volkswirtschaft für die solidarische Finanzierung des Medizinbetriebs geleuchtet - wobei die Erleuchtung, die aus ihren Beiträgen erwachsen kann, auch daraus resultiert, daß sie jenseits aller Interessensgebundenheit nach bestem (vorläufigen) Wissen den Stand der Dinge und ihre zukünftige Entwicklung darstellen können.

So ist die Entprivatisierung der Krankheit und die Kollektivierung des Krankheitsrisikos in der Menschheitsgeschichte mit der Einführung der gesetzlichen Krankenversicherung vor 110 Jahren sehr jungen Datums und überdies keineswegs sakrosankt. Der Versuch, Gesundheit zu objektivieren und einheitlich zu definieren, ist bislang nicht gelungen und wird aus Arnolds Sicht auch künftig am Freiheitswillen des Menschen scheitern. Dies gilt vor 
allem dann, wenn biopsychosoziale Komponenten in die Definition der Gesundheit einbezogen werden. So, wie vor 110 Jahren die sehr rudimentäre kollektive Absicherung des Gesundheitsrisikos (nur bei industriell beschäftigten Arbeitnehmern) primär aus Zweckmäßigkeitsgesichtspunkten geschaffen wurde, sei nun nachdem die Zweckmäßigkeits- von der Gerechtigkeitsethik überlagert worden ist - zu überlegen, ob unter den veränderten Bedingungen künftig die Reprivatisierung des Gesundheitsrisikos im Gemeinschaftsinteresse sei. Denn gemessen am harten Indikator Lebenserwartung gibt es nach Arnolds Analyse offenbar keine gesetzmäßige Abhängigkeit zwischen der Menge medizinischer Leistungen und dem Maß an öffentlicher Gesundheit. Das "Gewohnte" muß also geändert werden -, und ohne merkliche soziale und individuelle Kosten wird dies nicht möglich sein, schon gar nicht in harmonisch verlaufenden Prozessen.

Nun mag sich die nationalstaatliche Politik dem entgegenstemmen, was ihr aber nach der Analyse von Frank Münnich nicht viel helfen wird. Die Globalisierung der Wirtschaft und die Flüchtigkeit des Kapitals entzieht einen erheblichen Teil wirtschaftlicher Entscheidungen der Beeinflußbarkeit durch den Nationalstaat.

Die Volkswirtschaften der westlichen Staaten stehen dabei alle vor den gleichen Problemen: Die Revolutionen in der Kommunikationsindustrie schaffen in ihr selbst zwar neue Arbeitsplätze, führen aber in vielen anderen Branchen zu drei Effekten:

- zur Rationalisierung durch Arbeitsersparnis,

- zur Entstehung neuer Arbeitsstrukturen, die nicht mehr standortgebunden sind,

- und zu neuen Lebensarbeitszeitkarrieren, die künftig weniger an feste Arbeitsplätze und unbefristete Arbeitsverträge gebunden sind und von werkvertragsähnlichen Verhältnissen sowie frei gewählter Freiberuflichkeit geprägt sind.

Die Machtverschiebung von Arbeit zu Kapital, von nationalen zu internationalen Organisationen sowie die brüchig werdenden konventionellen Arbeitsverhältnisse reißen offene Flanken in die Finanzierung der Sozialversicherung, die an konventionelle Beschäftigungstypen geknüpft ist. 
Diese für die Sozialversicherung fundamental bedrohlichen Entwicklungen haben freilich nicht ausschließlich zu düsterer Endzeitstimmung, sondern auch zur Ideensuche für einen neuen Aufbruch geführt. Die Erfahrungen mit inzwischen 25 Jahre dauernder interventionistischer Kostendämpfungspolitik mit etlichen Verschiebungen zwischen den Sozialversicherungssystemen und den Staatshaushalten haben Lerneffekte ausgelöst. So ist die vorliegende Dokumentation des Symposions der Beleg dafür, daß Diskussionen um Reformoptionen auch unter den Praktikern und damit unter den Betroffenen mit einer neuen Offenheit stattfinden - sicherlich eine neue Dimension für Reformen im Gesundheitswesen:

- Die Vertretung des eigenen Interessensstandpunktes steht bei den Betroffenen nicht mehr allein im Vordergrund der Argumentation; man hat begriffen, daß reine Abwehrhaltungen längst nicht mehr den Status quo sichern können, geschweige denn Fortschritte bringen.

- Regelrecht auffällig ist der Verzicht darauf, konkrete Reformendpunkte zu setzen. Das ist natürlich einerseits bedauerlich, weil niemand mehr so genau weiß, wohin am Ende die Reformreise gehen wird. Auf der anderen Seite ist dies jedoch das realistische Eingeständnis, daß sogenannte Reformendpunkte, also der Zustand eines dauerhaften Optimums, eigentlich nur IIlusion sind.

- Statt dessen zeichnet sich eine neue Kultur des Reformierens ab, die durch das Gesundheitsstrukturgesetz 1993 sowie durch das 1. und 2. GKV-Neuordnungsgesetz 1997 entstanden ist: in Wettbewerbsprozessen nach alternativen Lösungswegen zu suchen; Versuch und Irrtum mit der Möglichkeit zur Korrektur, zum Lernen und zum Kopieren des Bewährten könnten wohl in Zukunft den Reformpfad des Gesundheitswesens charakterisieren. Beunruhigend daran mag sein, daß es hierbei keine Gewißheiten gibt; beruhigend daran ist jedoch, daß die Chancen für Dogmatiker und die von innen suggerierten ScheinGewißheiten sinken.

So hat das Symposion denn auch die Schwergewichte auf die pragmatische Seite künftiger Reformoptionen gelegt und dies für drei Elemente der gesundheitlichen Versorgung diskutiert: die Finanzierungsbasis des Gesundheitswesens, den Prozeß der Lei- 
stungserstellung und die Neuordnung von Solidarität und Subsidiarität.

Wer sich freilich bei der Lektüre dieses Buches ein schlichtes $\mathrm{Pa}$ tentrezept erhofft, dürfte enttäuscht werden: Isolierte Veränderungen bringen weder auf der Finanzierungsseite noch in der Organisation der Leistungserstellung noch durch Ausgrenzung von Leistungen dauerhaft "durchschlagende" Wirkungen. Sehr eingehend haben sich die Symposionsteilnehmer - natürlich auch unter dem Blickwinkel der jeweils von innen zu vertretenden Interessen - mit den Neben- und Fernwirkungen von Systemveränderungen befaßt. So würde beispielsweise die Anhebung der Versicherungspflichtgrenze und der Beitragsbemessungsgrenze der gesetzlichen Krankenversicherung auf das 100-Prozent-Niveau der Rentenversicherung den Beitragssatz der Krankenkassen im Schnitt um knapp 0,7 Prozentpunkte entlasten können. Gemindert wird dieser Effekt jedoch um Übergangsprobleme: etwa der der schon jetzt Privatversicherten, in deren Versicherungsschutz nicht eingegriffen werden könnte. Weitere Nebeneffekte: Der Entlastung durch einen gesunkenen Beitragssatz steht die stark steigende Grenzbelastung bei mittleren und höheren Einkommen gegenüber. Weil schließlich die private Krankenversicherung Mitglieder verliert, die in der ambulanten ärztlichen Versorgung und bei der Klinikbehandlung höhere Honorare und höhere Erstattungen als die gesetzliche Krankenversicherung leisten, könnte der Nettofinanzierungseffekt für das Gesundheitswesen insgesamt aufgrund verminderter Subventionsleistungen aus der PKV gegen Null gehen oder gar negativ werden.

Euphorie, aber auch energischen Widerstand haben die Modellversuche und Strukturverträge, wie sie nach dem 2. GKV-Neuordnungsgesetz möglich geworden sind, ausgelöst. Gesetzgeber und Krankenkassen sehen darin die Chance, die Sektorierung im deutschen Gesundheitswesen aufzubrechen und Rationalisierungspotentiale auszuschöpfen. Für die Vertragsärzte und deren Organisationen wird damit ein Tabu gebrochen, wonach es bislang als unärztlich oder sogar unethisch galt, an eingesparten veranlaßten Leistungen zu „verdienen“. Die Materie der Modellversuche und Strukturverträge, wie sie in den Beiträgen von Gerd Glaeske und Wolfgang Schmeinck dargestellt wird, ist allerdings vielfältiger als die ersten recht grobschlächtigen Bonusverträge glauben machen: Sie sollen primär den stationären Sektor entlasten (Glaeske) und dort Ausgaben sparen, wogegen allerdings 
der gegenwärtige Mangel an Leistungs- und Abrechnungstransparenz sowie die Machtverteilung zwischen den Vertragspartnern und den Leistungssektoren spricht. Einer Stärkung der ambulanten Versorgung müßten die Arzneimittelhersteller im Grunde positiv gegenüberstehen, die freilich angesichts konkreter Vertragsgestaltungen befürchten, als erste Opfer neuer Kostendämpfungsstrategien zu sein. Asymmetrische Transparenz könnte sich damit durchaus gegen diejenigen auswirken, deren Leistungen und Kosten am ehesten berechenbar sind. Grundsätzliche Konflikte sind aber auch zwischen Ärzten und Kassen denkbar: Modellversuche und Strukturverträge zielen darauf $a b$, daß Ärzte stärker als bisher gesundheitliche Leistungen integrativ organisieren. Nichts läuft aber ohne die Organisations- und Vertragskompetenz der Kassenärztlichen Vereinigungen, und diese wiederum neigen zu möglichst einheitlichen und kassenübergreifenden Lösungen. Das läuft freilich dem Bestreben der Krankenkassen nach Kassenindividualität zuwider und konterkariert wettbewerbliche Suchprozesse. Und am Endpunkt aller Bemühungen steht der Versicherte und Patient, der einstweilen die Vorteilhaftigkeit neuer Versorgungsformen am wenigsten erkennen kann - zumindest dann nicht, wenn inm nicht ein gehöriger Beitragsnachlaß versprochen wird.

Noch weitaus schwieriger scheint es wohl, Solidarität und Subsidiarität in ein neues Verhältnis zueinander zu bringen. Zwar lassen sich zahllose Belege dafür finden, daß Menschen sehenden Auges gesundheitliche Risiken in Kauf zu nehmen bereit sind und für diese Vergnügungen - wie Hochrisikosport oder Urlaub in den Tropen - auch kräftig bezahlen; ein Verschuldensprinzip für selbstverursachte Erkrankungen, dies war ein wichtiger Konsens des Symposions, wird durchweg abgelehnt. Auch die Ausgrenzung von Leistungen unter medizinischen Aspekten wird als ausgesprochen problematisch angesehen. Denn bislang sind alle Versuche gescheitert, objektiv und ex ante allgemein die NichtNotwendigkeit einer medizinischen Leistung zu bestimmen. Mit diesem Unvermögen wird dann allerdings auch eingestanden, daß jede Ausgrenzung von Leistungen Willkürelemente und letztlich ein politisches Werturteil enthält, das auf einem breiten Konsens der Gesellschaft beruhen muß, jedoch wissenschaftlich nicht ableitbar ist. Die Vorstellung nämlich, die Medizin oder die Ärzteschaft könne aus ihrem Sachverstand definieren, welche Leistungen allgemein verzichtbar seien, scheitert zum einen am 
Sachverstand (oder seinem Mangel), zum anderen aber sicher an der interessenbedingten Subjektivität der Medizin.

So bleibt am Ende des Symposions und auch am Ende der Lektüre dieser Dokumentation sicher ein Stück Ratlosigkeit - ein Bündel offener Fragen, die zur Fortsetzung der Reformdiskussion genügend Grund geben. 


\section{Begrüßung}

Manfred Albring

Lieber Herr Professor Wille, meine sehr geehrten Damen und Herren,

zu unserem „2. Bad Orber Gespräch über kontroverse Themen im Gesundheitswesen" darf ich Sie im Namen der Schering Aktiengesellschaft sehr herzlich begrüßen.

Ich hoffe, daß Sie eine angenehme Anreise hatten, mit Ihrer Unterkunft zufrieden sind und hier in Bad Orb eine Arbeitsatmosphäre vorfinden, die eine aufgeschlossene, sachbezogene und zielorientierte Diskussion erleichtern wird.

Mit diesem 2. Workshop setzen wir den Versuch fort, gesundheitspolitische Probleme zu analysieren und Lösungsansätze zu erarbeiten. Im vergangenen Jahr haben wir uns mit dem schillernden und interpretationsfähigen Begriff der Innovation und seinen spezifischen Nuancierungen in der Arzneimitteltherapie auseinandergesetzt. Die Dokumentation dieses Workshops hat bei Partnern im Gesundheitswesen Interesse und Resonanz gefunden: Relevant könnte dies bei Richtgrößenvereinbarungen werden, bei denen Innovationen einen besonderen Stellenwert haben.

Im Herbst dieses Jahres stehen wir vor neuen Herausforderungen, die wir auch zum Thema dieses Workshops machen wollen. Nicht primär die Ausgaben der gesetzlichen Krankenversicherung, sondern die wegbrechende Einnahmenbasis verursacht den Hauptteil der Probleme. Das 1. Halbjahr 1997 hat in der gesetzlichen Krankenversicherung mit einem Defizit von $4 \mathrm{Mrd}$. DM abgeschlossen, obwohl die Ausgaben nahezu konstant geblieben sind. Im 2. Halbjahr könnte der Ausgleich allein dadurch geschaffen werden, weil ab der Jahresmitte die Zuzahlungen so stark wie nie gestiegen sind. Für 1998 prognostizieren die wirtschaftswissenschaftlichen Forschungsinstitute ein Wirtschaftswachstum von real 2,8\%. Das reicht aber bei weitem nicht aus, bei stark steigender Produktivität Beschäftigungswirkung zu erzielen. Die Zahl der Arbeitslosen könnte also aller Voraussicht nach weiter steigen, und in Kombination damit werden sehr maßvolle Tarifab- 
schlüsse die Einnahmenbasis aller Sozialversicherungen weiter schmälern - oder zumindest nicht verbreitern.

Meine Damen und Herren, die Gewitterfront im Gesundheitswesen ist damit von folgenden Spannungen bestimmt:

- durch den Fortschritt der diagnostischen und therapeutischen Möglichkeiten. Meistens wird dadurch zwar nicht eine kausale Heilung von Krankheiten erreicht, jedoch ein lebenswertes Leben mit behandelbar gewordenen Krankheiten,

- durch die zunächst allmähliche, ab dem Jahr 2005 stärker steigende Überalterung der Gesellschaft,

- durch die langfristige Gewöhnung der Bevölkerung an eine fast Vollkaskoversicherung, die meistens auch kleinere und kleinste Lebensrisiken abdeckt, und schließlich

- durch die vorhin skizzierten Finanzierungsprobleme der gesetzlichen Krankenversicherung, die durch Einnahmenrückgänge verursacht werden und damit nicht einen konjunkturellen und vorübergehenden Schwächeanfall des Systems darstellen.

Bei dieser Ausgangssituation versucht das 2. NOG die Weichen neu zu stellen, indem es die Eigenverantwortung der Bürger in zunehmendem Maß in die Gesundheitsversorgung mit einbezieht. Auch der Versicherte selbst möchte als mündiger und kritischer Patient zunehmend selbst über seinen Umgang mit Gesundheit und mit Informationen über seinen Gesundheitszustand mitentscheiden.

Angesichts dieser Situation nimmt es nicht wunder, wenn sich beispielsweise die Kassenärztliche Bundesvereinigung anläßlich ihres Symposions am 20. und 21. Oktober in Königswinter mit der Neuordnung des Leistungskataloges in der gesetzlichen Krankenversicherung beschäftigt hat und zu dem Schluß kommt, daß die Leitidee der solidarischen Krankenversicherung zukünftig „aus ökonomischen Gründen eine notwendige Straffung des Leistungskataloges und Begrenzung auf das vom Gesetzgeber vorgegebene notwendige Maß" sein sollte.

Das ebenfalls kürzlich verabschiedete Berliner Thesenpapier, das in Zusammenarbeit mit Vertretern der Krankenkassen, der Kassenärztlichen Vereinigung Berlin, der Gewerkschaften und Fach- 
beamten der Senatsverwaltung unter dem Vorsitz von Frau Hübner, Senatorin für Gesundheit und Soziales, erarbeitet wurde, kommt zu noch deutlich weitergehenden Empfehlungen: Man beabsichtigt nämlich, nach neuen Finanzierungsalternativen für die gesetzliche Krankenversicherung zu suchen:

- eine stärkere solidarische Finanzierung, die alle Einkommen unter Wegfall der Beitragsbemessungsgrenzen mit einbeziehen soll,

- einen Umbau der Mittelaufbringung zur Senkung der Lohnnebenkosten für Arbeitgeber und Arbeitnehmer und

- gegebenenfalls Überlegungen, wie eine Finanzierung neuer, als medizinisch notwendig legitimierter Leistungen (ohne Erhöhung der Lohnnebenkosten) auch zu Lasten von Leistungen in der GKV, die als verzichtbar erachtet werden, erreicht werden kann.

Insofern befindet sich das 2. Bad Orber Gespräch über Reformoptionen im Gesundheitswesen auch zeitlich in einem passenden Rahmen. Darüber hinaus, so hoffe ich, werden wir durch die Bearbeitung der Themenschwerpunkte

- „Einnahmenentwicklung der GKV und Finanzierungsoptionen"

- "Chancen und Risiken von Modellvorhaben und Strukturverträgen"

- "Der Leistungskatalog im Lichte des Solidaritätsprinzips"

versuchen, das Gesamtthema in einem größeren Kontext zu bearbeiten. Sicherlich werden wir es nicht schaffen, in der uns zur Verfügung stehenden Zeit die Quadratur des Kreises zu erreichen und endgültige politisch verwertbare Lösungen anzubieten. Aber die versammelte Kompetenz der anwesenden Experten läßt erwarten, daß dieser Schering-Workshop eine qualitativ hochwertige Auseinandersetzung mit den anstehenden Themen leisten wird. Ich bin sicher, daß unsere Arbeit eine publikationswürdige Diskussionsgrundlage bilden wird, die bei vielen Entscheidungsträgern im Gesundheitswesen Denkanstöße auslösen kann.

Persönlich freue ich mich ganz besonders, daß es uns gelungen ist, für die diesjährige Veranstaltung Herrn Professor Arnold als Festredner gewonnen zu haben. Innen Herrn Professor Arnold 
vorstellen zu wollen, hieße Eulen nach Athen tragen. - Deshalb kann ich nur für mich sprechen. - In den $2 \frac{1}{2}$ Jahren, in denen ich mich mit dem Gesundheitswesen beschäftige, habe ich Professor Arnold als messerscharfen Analytiker und visionären Wissenschaftler erlebt, der darüber hinaus auch die Größe besitzt, mit eigenen früheren Aussagen kritisch umzugehen. Deshalb darf ich schon jetzt die Überzeugung äußern, daß sein Vortrag mit dem wertneutralen Titel „Entwicklungsperspektiven der medizinischen Versorgung" eine optimale Einstimmung für den Workshop schaffen wird.

Damit möchte ich jetzt der Veranstaltung trotz unterschiedlichster Interessenslagen nicht nur einen kontroversen, sondern auch zielorientierten und fairen Verlauf wünschen und darf das Wort an unseren Chairman weitergeben. 


\section{Aktuelle Anlässe und Ansatzpunkte der Reformdiskussionen im Gesundheitswesen}

Eberhard Wille

\section{Zum Prozedere der Bad Orber Gespräche}

Vielen Dank Herr Dr. Albring. Meine sehr geehrten Damen und Herren,

zunächst möchte auch ich Sie sehr herzlich begrüßen und Ihnen danken, daß Sie gut $11 / 2$ Tage opfern, um an den diesjährigen Bad Orber Gesprächen teilzunehmen. Ich weiß dies um so mehr zu schätzen, als Sie mit Ihrer Teilnahme hohe Opportunitätskosten in Form von entgangenen alternativen Verwendungen Ihrer knappen zeitlichen Ressourcen in Kauf nehmen. Wie Herr Dr. Albring schon erwähnte, handelt es sich um die zweite Veranstaltung dieser Art, und die Resonanz auf die Publikation der letztjährigen Bad Orber Gespräche über „Innovationen in der Arzneimitteltherapie ${ }^{\text {" }}$ hat uns ermutigt, das damalige Prozedere im Prinzip beizubehalten.

Im Anschluß an den Festvortrag von Herrn Professor Arnold über "Entwicklungsperspektiven der medizinischen Versorgung" folgen jeweils drei Kurzvorträge zu unseren drei zentralen Themenkomplexen:

- „Finanzierungsoptionen“ (Frau Walzik, Frau Prof. Pfaff, Prof. Münnich)

- „Strukturverträge und Modellvorhaben“ (Dr. Glaeske, Dr. Krimmel, Herr Schmeinck)

- „Das Solidaritätsprinzip“ (Dr. Dr. Ehlers, Herr Schirmer, Dr. von Stillfried)

Diese Statements dienen als thematischer Einstieg in die Diskussionen der drei entsprechenden Arbeitsgruppen, die unter der Leitung von Herrn Professor Cassel, Herrn Schulte und Herrn Firnkorn stehen. Den Leitern der Arbeitsgruppen obliegt auch die

1 vgl. Manfred Albring und Eberhard Wille (Hrsg.), Innovationen in der Arzneimitteltherapie. Definition, medizinische Umsetzung und Finanzierung. Verlag Peter Lang, Frankfurt et al. 1997 
Aufgabe, die Argumentationslinien und Ergebnisse dieser Diskussionen im Plenum nachzuzeichnen. Auf dieser Informationsgrundlage findet dann eine übergreifende Diskussion der gesamten Thematik im Plenum statt. Zum Abschluß dürfen Sie mich dann bei dem ambitiösen Unterfangen „bewundern“, den Veranstaltungsverlauf in seinen Grundzügen zusammenzufassen.

Die Bad Orber Gespräche intendieren in mehrfacher Hinsicht eine ganzheitliche Betrachtungsweise von kontroversen Themen im Gesundheitswesen. Um einer isolierten und interessenbezogenen Argumentation vorzubeugen, diskutieren z. B. im Rahmen dieser Veranstaltung Vertreter von Kassenärztlichen Vereinigungen, verschiedenen Krankenkassen, der privaten Krankenversicherung, der pharmazeutischen Industrie, der Wissenschaft, den Medien, der Ministerialbürokratie und der Politik miteinander. Sodann erlaubt in den meisten Fällen nur ein interdisziplinärer Ansatz, der medizinische, pharmakologische, juristische und ökonomische Aspekte berücksichtigt, eine adäquate Behandlung von Problemen bzw. strittigen Fragen im Gesundheitswesen. Sachliche Diskussionen unter Experten tendieren insofern zu inhaltlichen Konvergenzen, als sie Verständnis für andere Ansätze und Meinungen wecken, die eigenen Standpunkte relativieren und in diesem Zusammenhang nicht selten extreme Positionen „abschleifen“. Unter diesem Aspekt zielt die Veranstaltung darauf ab, im Hinblick auf allfällige gesundheitspolitische Reformoptionen Übereinstimmungen, unterschiedliche, aber konvergierende Ansichten und weiterhin divergierende Meinungen herauszuarbeiten.

\section{Nur kurzfristiger oder struktureller Einbruch der Finanzierungsbasis?}

Ohne die fiskalischen Effekte der jüngsten Neuordnungs- bzw. Reformgesetze würden der gesetzlichen Krankenversicherung (GKV) schon im Jahre 1997 wieder Budgetdefizite und damit steigende Beitragssätze drohen. Dabei scheint die GKV momentan weniger an einem exzessiven Ausgabengebaren als an einer notleidenden Finanzierungsbasis zu kranken. Der nur mäßige Anstieg der beitragspflichtigen Einnahmen vermag nicht einmal mit einer vergleichsweise moderaten Ausgabenentwicklung Schritt zu halten. Diese Finanzierungsprobleme wurzeln einmal in den häufig beklagten „Verschiebebahnhöfen“, die innerhalb der Teilsysteme der sozialen Sicherung in den letzten Jahren fast immer zu Lasten der GKV-Einnahmen gingen. Für die abbröckelnde Finan- 
zierungsgrundlage der GKV zeichnen aber auch das schwache Wirtschaftswachstum, die hohe Arbeitslosigkeit und die gewandelten Arbeitsverhältnisse verantwortlich. Diese Einflußfaktoren verhindern eine hinreichende Zunahme der Arbeitsentgelte, die als Grundlohnsumme den zentralen Teil der Beitragsbemessungsgrundlage bilden.

Die wirtschaftspolitische Problematik steigender Beitragssätze in der GKV veranschaulicht aus der Perspektive der öffentlichen Abgabenentwicklung Abbildung 1. Während die sog. gesamtwirtschaftliche Steuerquote, d. h. der Anteil des Steueraufkommens am Bruttoinlandsprodukt, in den letzten 40 Jahren praktisch konstant blieb, nahm die sog. Sozialabgabenquote im Beobachtungszeitraum signifikant zu. Die Sozialabgaben stiegen vor allem von 1965 bis 1976 und von 1990 bis 1995 stärker an als das Bruttoinlandsprodukt. Setzt sich dieser Trend der letzten 30 Jahre künftig fort, dann dominieren in absehbarer Zeit innerhalb der öffentlichen Abgaben die Sozialabgaben in quantitativer Hinsicht die Steuern. Bei allen Einwänden, die sich unter allokativen Aspekten gegen das Ziel der Beitragssatzstabilität vorbringen lassen, gewinnt dieses Postulat im Licht der öffentlichen Abgabenentwicklung schon eine gewisse Berechtigung.

Abb. 1: Steuer- und Sozialabgabenquote in der BRD 1950-1995

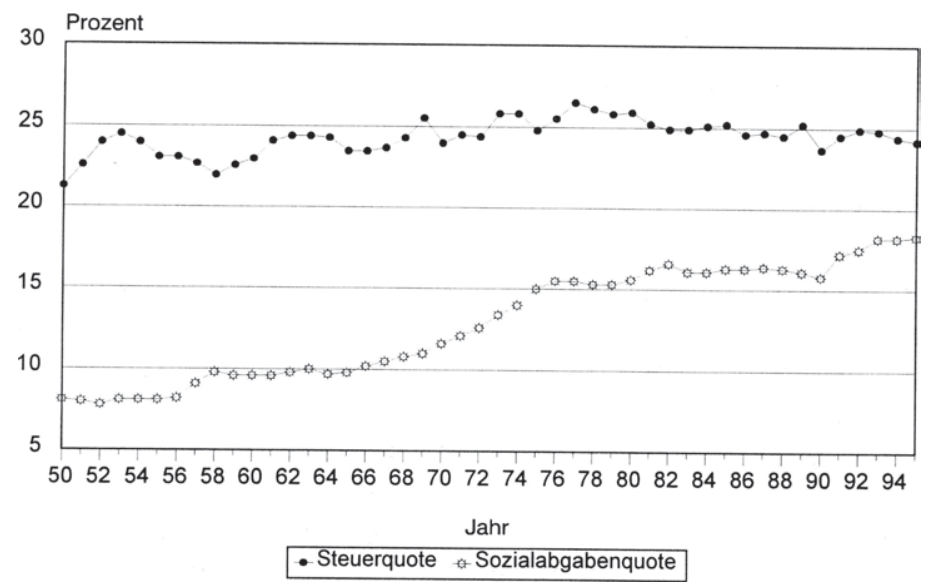

Quelle: Zusammengestellt und errechnet aus Statistisches Bundesamt [1989, 1991a, 1991b, 1995, 1996]. 
Die Wachstumsschwäche der Einnahmenbasis von GKV und von gesetzlicher Rentenversicherung (GRV) deutet Abbildung 2 an. Danach stagnierte der Anteil der GKV-Beiträge am Bruttoinlandsprodukt (BIP) von 1976 bis 1991, was für den entsprechenden Ausgabenanteil der GRV sogar für den Zeitraum von 1974 bis 1993 gilt. In dieser Zeitspanne nahmen jedoch die Beitragssätze von GKV und GRV zu, denn die beitragspflichtigen Einnahmen stiegen schwächer als das BIP. Die im Vergleich zum BIP unterdurchschnittliche Wachstumsrate der Beitragsbemessungsgrundlage bzw. der Lohnsumme geht nicht zuletzt auf die zunehmende Arbeitslosigkeit zurück, die in Abbildung 2 in dem steigenden Anteil der Beiträge zur Arbeitslosenversicherung zum Ausdruck kommt. Die GKV erhält zwar nicht nur von den unselbständig Beschäftigten, sondern auch von Selbständigen und sonstig freiwillig Versicherten sowie von Rentnern, Arbeitslosen und anderen Transferempfängern bzw. deren Versicherungsträgern Einnahmen. Die Substitution des Arbeitsentgeltes durch eine andere Bemessungsgrundlage führt jedoch bei - mit Ausnahme des Krankengeldes - unverändertem Leistungsanspruch zumeist zu deutlichen Mindereinnahmen.

Abb. 2: Die Entwicklung der Sozialabgaben in Relation zum BIP nach Sozialversicherungsträgern

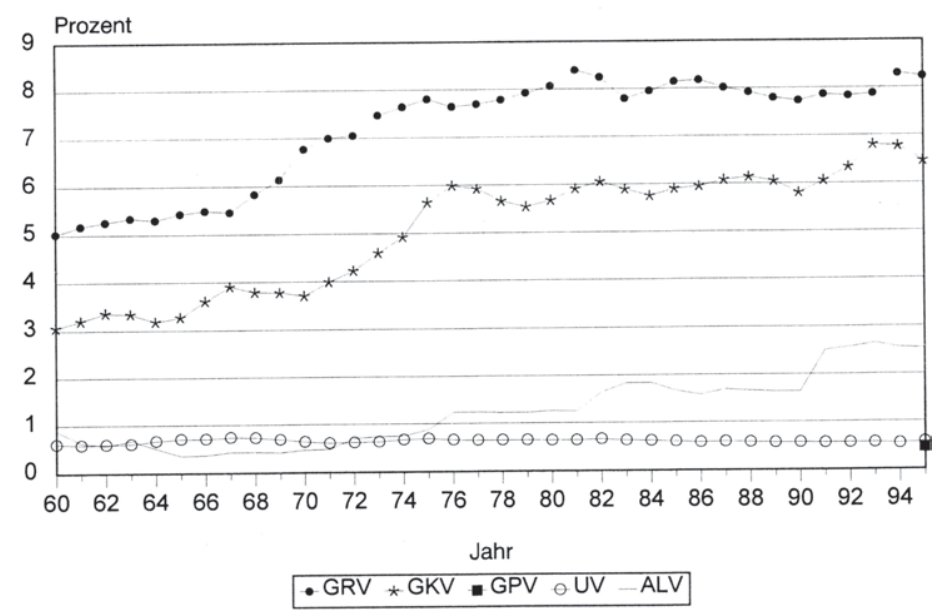

Quelle: Zusammengestellt und errechnet aus Statistisches Bundesamt [1989, 1991a, 1991b, 1995, 1996]. 
Die erwähnte temporäre Konstanz des Anteils der GKVAusgaben am BIP stellte sich nicht automatisch im Zuge einer endogenen Entwicklung ein, sondern bildet das Resultat zahlreicher diskretionärer staatlicher Eingriffe. Mit Hilfe von sog. Kostendämpfungsmaßnahmen bzw. Gesundheitsreformen gelang es - wenn auch jeweils nur für eine kurze Frist -, die Wachstumsrate der GKV-Ausgaben unter die Steigerungsrate der beitragspflichtigen Einnahmen zu drücken und so die Beitragssätze zeitweilig zu stabilisieren. Abbildung 3 veranschaulicht diesen Sachverhalt und deutet zugleich auf die zentralen diskretionären staatlichen Eingriffe hin:

- das Krankenversicherungs-Kostendämpfungsgesetz vom 27.06.1977

- das Kostendämpfungs-Ergänzungsgesetz vom 22.12.1981

- das Gesundheitsreformgesetz vom 20.12.1988 und

- das Gesundheitsstrukturgesetz vom 21.12.1992.

Abb. 3: Jährliche Veränderungsraten der Ausgaben der GKV für Gesundheit ${ }^{1)}$ und der beitragspflichtigen Einnahmen (Grundlohnsumme) ${ }^{2)}$

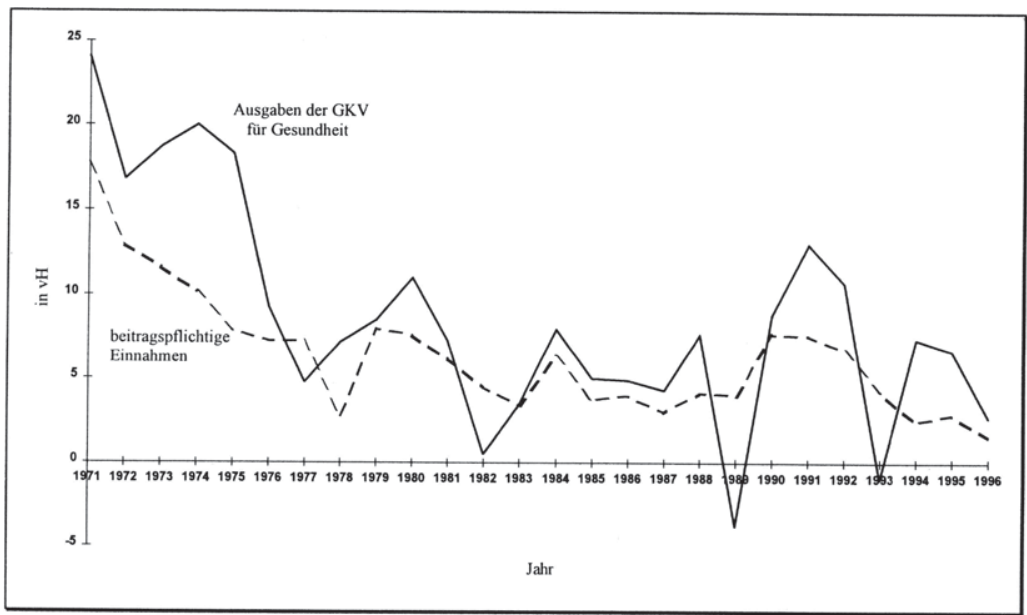

1) Ab 1995 wird Berlin-Ost den alten Bundesländern zugeordnet.

2) Ab 1978 geänderte Berechnungsmethode, schränkt Vergleichbarkeit mit vorangehenden Jahren ein.

Quelle: Zusammengestellt und errechnet aus: Sachverständigenrat für die Konzertierte Aktion im Gesundheitswesen [1994], Bundesministerium für Arbeit und Sozialordnung [1997] und Bundesministerium für Gesundheit [1997]. 
Diese sog. Kostendämpfungsmaßnahmen konnten für einen beachtlichen Zeitraum den Anteil der GKV-Ausgaben am BIP konstant halten, aber wegen der schwachen Einnahmenentwicklung einen Anstieg der Beitragssätze nicht verhindern. Setzt sich dieser Trend künftig fort, daß die Beitragsbemessungsgrundlage im Wachstum nicht mit dem BIP Schritt zu halten vermag, steht die GKV vor folgendem Dilemma: Entweder orientieren sich ihre Ausgaben am Wachstum des BIP bei dann zwangsläufig steigenden Beitragssätzen, oder die Beitragssätze bleiben stabil. Dies setzt aber voraus, daß die GKV-Ausgaben schwächer zunehmen als das BIP. Die erste Alternative führt offensichtlich bei den Arbeitgebern zu einer Erhöhung der Lohnnebenkosten und bei den Arbeitnehmern zu einer Verminderung ihres verfügbaren Einkommens. Die zweite Alternative engt vor allem bei einer wenig prosperierenden Wirtschaftsentwicklung, die schon mit einem bescheidenen Wachstum des realen BIP einhergeht, den Finanzierungsspielraum der GKV stark ein. Dieser dürfte dann rebus sic stantibus, $d$. $h$. ohne neue Reformmaßnahmen, kaum ausreichen, um künftig die zentralen ausgabenseitigen Herausforderungen, wie $\mathbf{z}$. B. den medizinischen Fortschritt und den demographischen Wandel, finanziell zu bewältigen.

Angesichts der skizzierten Entwicklungstrends steht zu befürchten, daß die Wachstumsschwäche der GKV-Einnahmenbasis nicht nur kurzfristiger Natur ist, sondern strukturell bedingt zumindest auf mittlere Frist anhält. Die Globalisierung der Wirtschaft und die Öffnung der osteuropäischen Staaten, die über relativ wenig Kapital, aber reichlich Arbeitskräfte verfügen, haben die Knappheitsverhältnisse zwischen den Produktionsfaktoren Arbeit und Kapital nachhaltig zuungunsten der Arbeitskraft verschoben. Die daraus resultierenden Preis- bzw. Lohn- und Mengenwirkungen beeinträchtigen umlagefinanzierte Versicherungssysteme, die sich primär auf die Arbeitsentgelte stützen, weitaus stärker als kapitalgedeckte Versicherungen. Zudem erodiert die Beitragsbemessungsgrundlage von GKV und GRV durch die zunehmende Auflösung der bisher üblichen Arbeitsverhältnisse, denn an die Stelle von dauerhafter Vollzeitarbeit treten befristete und/oder geringfügige Beschäftigungsverhältnisse, Teilzeitarbeit und (Schein) Selbständigkeit. Insofern erscheint es unwahrscheinlich, daß die GKV auf mittlere Frist das in $\S 71$ SGBV verankerte Postulat der Beitragssatzstabilität ohne weitere Reformschritte auf der Einnahmen- und/oder Ausgabenseite zu verwirklichen vermag. 


\section{Globale Reformoptionen}

Sofern im Rahmen der GKV Budgetdefizite drohen, existieren grundsätzlich folgende Finanzierungsmöglichkeiten bzw. globale Reformoptionen:

- Ausschöpfung von Wirtschaftlichkeitsreserven,

- Beitragssatzerhöhungen,

- zusätzliche Mittelaufbringung durch Änderung der Beitragsgestaltung,

- Eingrenzung des Leistungskataloges und

- Verlagerung der Finanzierung auf andere Ausgabenträger, wie z. B. öffentliche Haushalte, andere Teilsysteme der sozialen Sicherung oder private Haushalte.

Diese globalen Finanzierungsmöglichkeiten können unabhängig von Budgetdefiziten auch dazu dienen, die Beitragssätze aufkommensneutral zu senken. Im Mittelpunkt der Arbeitsgruppe 1, die sich mit den "Finanzierungsoptionen" beschäftigt, stehen in erster Linie die zusätzliche Mittelaufbringung durch Änderung der Beitragsgestaltung und daneben die Verlagerung der Finanzierung auf andere Ausgabenträger. Die Thematik der Arbeitsgruppe 2 "Strukturverträge und Modellvorhaben" ließe sich als ein Instrument zur Ausschöpfung von Wirtschaftlichkeitsreserven interpretieren. Die Tatsache, daß diese Arbeitsgruppe den größten Zuspruch der Teilnehmer fand, zeugt von einer gewissen Zuversicht, das Rationalisierungspotential innerhalb der GKV in einem relevanten Umfang ausschöpfen zu können. Bei der Frage einer Eingrenzung des Leistungskataloges bildet das Solidaritätsprinzip, über das die dritte Arbeitsgruppe diskutiert, ein wesentliches Beurteilungskriterium. Schließlich stellen Beitragssatzerhöhungen, die im geltenden System bei Budgetdefiziten stattfinden, die Bezugs- bzw. Referenzbasis für eine Beurteilung der diversen Reformoptionen dar.

Von diesen globalen Reformoptionen kommt der Ausschöpfung von Wirtschaftlichkeitsreserven in normativer Hinsicht allererste Priorität zu. Unter diesem Aspekt läßt sich die Forderung „Rationalisierung statt Rationierung" um den Grundsatz „Ausschöpfung von Wirtschaftlichkeitsreserven vor Inanspruchnahme neuer $\mathrm{Fi}$ nanzierungsquellen" ergänzen. Obgleich das deutsche Gesundheitswesen unbeschadet seines recht guten internationalen Rufes noch beachtliche Wirtschaftlichkeitsreserven aufweist, hilft der 
Appell, zuerst einmal das vorhandene Rationalisierungspotential zu erschließen, bei der kurzfristigen Finanzierung von Budgetdefiziten in der Realität kaum weiter. Die Ausschöpfung des Rationalisierungspotentials setzt in vielen Fällen strukturelle Reformen voraus, die zumeist erst mit einer zeitlichen Verzögerung zu Ressourceneinsparungen und/oder Outcome-Erhöhungen führen.

Die Effekte von Reformansätzen können auch deshalb hinter den Erwartungen zurückbleiben, weil die Beteiligten Einkommenseinbußen befürchten und zu Ausweich- bzw. Gegenreaktionen greifen. In diesem Kontext setzen auch die methodischen Probleme, bei dienstleistungsintensiven Tätigkeiten die Outputs bzw. die medizinischen Outcomes zu messen und verursachungsgerecht zuzurechnen, Planungsverfahren und kontrollorientierten Methoden gewisse Grenzen. Unbeschadet aller Bemühungen um eine Ausschöpfung von Wirtschaftlichkeitsreserven verbleibt daher im Dienstleistungsbereich beinahe zwangsläufig ein Bodensatz an Ineffizienzen und Ineffektivitäten. Schließlich bewirken erfolgreiche Maßnahmen zur Verbesserung von Effizienz und Effektivität der Gesundheitsversorgung, die z. B. auf Maßnahmen zur Qualitätssicherung, wie z. B. Leitlinien bzw. Guidelines, zurückgehen, nicht nur Ausgabensenkungen, sondern auch OutcomeErhöhungen. Letztere besitzen unter normativen Aspekten keineswegs einen geringeren Wert als Kosteneinsparungen, vermögen aber keinen Beitrag zur Finanzierung von Budgetdefiziten zu leisten.

Die Ausschöpfung von Wirtschaftlichkeitsreserven im Gesundheitswesen stellt somit eine gesundheitspolitische Daueraufgabe dar, die aber - vor allem für den Fall einer weniger günstigen Wirtschaftsentwicklung - vorsorgliche Überlegungen im Hinblick auf andere globale Finanzierungsoptionen nicht ersetzen kann. Neben den aufgelisteten globalen Finanzierungsoptionen, die bei erwarteten Budgetdefiziten in erster Linie auf das zukünftige $\mathrm{Ni}$ veau von Einnahmen und Ausgaben der GKV abzielen, steht bei gegebenem Ausgabenvolumen, d. h. unter der Annahme der Aufkommensneutralität, auch die derzeitige Finanzierungsstruktur zur Diskussion. Dabei kann es u. a. um

- die paritätische Finanzierung der Beiträge zwischen Arbeitgebern und Arbeitnehmern,

- eine Differenzierung der Beitragssätze zugunsten niedriger Einkommensklassen sowie 
gehen. Bei weiterer Interpretation fallen hierunter im Sinne der "inneren Finanzierungsstruktur" auch die Vergütungs- und Preissysteme der Leistungserbringer. Da letztere neben der Preisauch die Mengenentwicklung der erbrachten Leistungen beeinflussen, bestehen innerhalb der GKV vielfältige Wechselwirkungen zwischen Umfang und Struktur von Einnahmen und Ausgaben.

Angesichts drohender Budgetdefizite infolge ungünstiger gesamtwirtschaftlicher Konstellationen betonen die derzeitigen $\mathrm{Re}$ formdiskussionen relativ stark den fiskalischen Aspekt von Finanzierungsoptionen. Unbeschadet der grundsätzlichen Berechtigung fiskalischer Erwägungen und der Bedeutung der Beitragssatzstabilität bilden in normativer Hinsicht allokative und distributive Aspekte bzw. Ziele die entscheidenden Beurteilungskriterien für die Analyse von Pro und Contra der einzelnen Finanzierungsoptionen. $\mathrm{Da}$ die Patienten die Gesundheitsleistungen weitgehend unabhängig vom Konjunkturverlauf benötigen, spielt die Stabilisierungsaufgabe bei der Einnahmen- und Ausgabengestaltung gegenüber allokativen und distributiven Aspekten als Zieldimension eher eine untergeordnete Rolle. Allerdings wurzeln die Finanzierungsprobleme der GKV derzeit primär in der allgemeinen Wirtschaftsentwicklung, so daß Maßnahmen, die z. B. zu einem Abbau der Arbeitslosigkeit beitragen, ihrerseits das Wachstum der Grundlohnsumme stärken und damit die Finanzierungsprobleme der GKV mildern. Die Einnahmen und Ausgaben der GKV hängen somit in starkem Maße von der allgemeinen Wirtschaftlichkeitsentwicklung ab, können diese aber umgekehrt auch in gewissen Grenzen beeinflussen.

Bei einer gesamtwirtschaftlichen Analyse der Vor- und Nachteile diverser Reformoptionen gilt es allerdings schon vorab in Rechnung zu stellen, daß alle Alternativen, insoweit sie der Abwendung eines drohenden Defizits dienen, an irgendeiner Stelle zu einer Minderung der Verfügungsgewalt über knappe Ressourcen und daher bei den Betroffenen zu Wohlfahrtsverlusten führen. Eine isolierte Betrachtung von Pro und Contra könnte daher zu einer Verwerfung einer jeden dieser Finanzierungsoptionen gelangen. Da aber ein drohendes Budgetdefizit zwangsläufig eine (zusätzliche) Finanzierung erfordert, erscheint nur eine vergleichende Bewertung dieser Optionen im Sinne einer Differential- 
analyse problemadäquat. Es geht also unter der Annahme von drohenden Budgetdefiziten nicht um das isolierte Pro und Contra einer Erhöhung der Beitragsbemessungsgrenze oder um eine einseitige Auflistung von Vor- und Nachteilen einer eventuellen Einschränkung der beitragsfreien Mitversicherung, sondern um einen differentiellen Vergleich dieser Optionen mit der geltenden Regelung steigender Beitragssätze. Die Diskussionen in den Arbeitsgruppen könnten dazu beitragen, einen breiten und sachlich fundierten Diskurs über diese zugegebenermaßen etwas dornige Thematik auszulösen. 


\section{Entwicklungsperspektiven der medizinischen Versorgung}

Michael Arnold

Ideen bewegen die Welt in die Ferne, aber Vorteile lenken die nächsten Schritte der Menschen, und der "heilige Hunger nach Gold", welcher in der Menschheit noch wirksamer ist als der idealste Trieb der Religion, wird uns immer daran erinnern, daß die Hălfte der Geschichte der gemeinen Materie angehört.

F. Gregorovious: Die Geschichte der Stadt Rom im Mittelalter, siebentes Buch

\section{Ausgangslage}

Der Entwurf eines Problemlösungsvorschlages erfolgt ebenso wie die Analyse irgendeines Sachverhaltes von gewissen Grundpositionen aus. Eine vorurteilslose Erfassung der Wirklichkeit bringt nichts, weil sie sich in der reinen Beschreibung erschöpfte und z. B. keine Abhängigkeiten, Gesetzmäßigkeiten oder Zusammenhänge der Elemente eines Gegenstandsbereiches zu erkennen erlaubte.

Die Grundpositionen können - sofern der Problemlösungsansatz und die Sachverhaltanalyse von einem anderen verstanden werden sollen - nicht voraussetzungslos eingenommen, sondern sie müssen ihrerseits begründet werden. Man kommt so leicht zu einem unendlichen Regreß, bis am Ende irgendwelche a priori-Annahmen stehen, die ihrerseits nicht weiter abgeleitet werden können. Über sie kann es keine sinnvolle Diskussion mehr geben, denn mit innen beginnen auf Ideologien beruhende Standpunkte, die nicht als Ergebnis eines rationalen Diskurses eingenommen, sondern im Laufe der Sozialisation übernommen werden.

Bei der Auseinandersetzung mit den im Vortragsthema enthaltenen Problemen bin ich von einigen Grundpositionen ausgegangen, die durch den Blick in die Vergangenheit und durch Erfahrungen in der Gegenwart gerechtfertigt werden können:

- Die zivilisatorische und soziale Entwicklung hat nicht zielgerichtet zum Status quo geführt, und der Status quo ist auch nicht der Endpunkt dieser Entwicklung. So wie vor 100 Jahren niemand das Ausmaß des politischen, ökonomischen, technologi- 
schen und sozialen Wandels voraussehen konnte, der in diesem Zeitraum stattgefunden hat, so ist niemand in der Lage, den der nächsten 100 Jahre zu antizipieren. So viel nur steht fest: Die Welt wird dann völlig anders sein als die uns vertraute, und zwar nicht notwendigerweise im Sinne eines Noch-mehr, Noch-besser, Noch-sicherer. Auch ganz andere Szenarien sind denkbar, es gibt kein Naturgesetz, daß das Leben unserem Verständnis entsprechend ständig angenehmer werden müßte, daß es nicht in eine andere Richtung gehen könnte, die zu gemessen an unseren heutigen Maßstäben - höchst unbefriedigenden Zuständen führt. Ein Weltuntergang wäre das deshalb noch nicht, sondern nichts anderes als die Folge der notwendigen Anpassung an veränderte Umstände.

- Speziell die Entprivatisierung von Krankheit und die dazu korrespondierende Kollektivierung des Krankheitsrisikos sind relativ jungen Datums. Wenn die den heutigen Versorgungssystemen zugrunde liegenden Prinzipien als sakrosankt hingestellt werden, so wird verkannt, daß beim Entstehen der auf diesen Prinzipien beruhenden Versorgungssysteme ganz andere Ziele verfolgt wurden, als jedem Patienten alle Leistungen verfügbar zu machen, für die nach Expertenmeinung ein Bedürfnis besteht. Die damalige GKV unterschied sich etwa bezüglich des Verhältnisses von Arbeitnehmer- und Arbeitgeberanteil am Beitrag, des anspruchsberechtigten Personenkreises, der gewährten Leistungen und der verfolgten Ziele in praktisch jeder Hinsicht von der uns vertrauten gesetzlichen Krankenversicherung.

- Der Ausbau des medizinischen Versorgungssystems zu seiner aktuellen Größe, Differenziertheit und Zweckbestimmung erfolgte unter dem Einfluß zahlreicher Gestaltungsfaktoren und keineswegs einfach zur Verwirklichung irgendwelcher abstrakten Normen. So wie in anderen Sozialsystemen auch und selbst im Alltagsleben läßt sich vieles sekundär mit ethischen Argumenten rechtfertigen, was in Wirklichkeit sehr profanen Zwecken dient. Dagegen ist grundsätzlich nichts zu sagen, denn am Ende kommt es auf das Ergebnis des Handelns und nicht auf die Motive an, die inm zugrunde liegen. Nur relativiert es manche Aussage, mit der ein erreichter Zustand vor Veränderungen geschützt werden soll.

- Das Bemühen, die medizinische Versorgung jedem ohne Rücksicht auf die individuelle Kaufkraft oder auch ein Selbstverschulden am Eintreten des Behandlungsanlasses in kollektiver 
Anstrengung zu gewährleisten, findet als das uns Gewohnte unreflektiert Zustimmung. Beim genaueren Nachdenken wird aber keineswegs offenbar, warum in diesem einen und einzigen Wirtschaftsbereich materielle Gleichheit bei der Bedürfnisbefriedigung angestrebt wird. Gerechtfertigt werden könnte die Verwirklichung materieller Gleichheit bei der medizinischen Versorgung, wenn Gesundheit unter allen Umständen und bei allen Menschen den höchsten Rang in der Hierarchie von Lebensgütern und Lebenswerten einnehmen würde und sie in gesetzmäßiger Weise von der medizinischen Versorgung abhinge. Diese Annahmen treffen indes nicht zu:

a) Gesundheit ist trotz gegenteiliger Behauptungen nicht das höchste aller Güter, sondern wird oft bereitwillig aufs Spiel gesetzt, wenn nur der damit verbundene Nutzen groß genug ist. Dabei wird ein unmittelbar erzielter Nutzen aufgrund einer Art psychologischer Diskontierung höher eingeschätzt als die langfristigen Kosten.

b) Es gibt nicht eine einheitlich definierte Gesundheit, die für alle Menschen gleich ist und etwa irgendeiner Art Norm entspräche, d. h. ein statistisches Konstrukt wäre.

So gibt es körperlich schwer behinderte Kranke, die sich als gesund empfinden, und Kranke können Höchstleistungen erbringen. Ein Mensch, der stets und ständig um seine Gesundheit besorgt ist, erscheint mit Recht als Psychopath. Konsequenterweise geht dann aber auch eine medizinische Versorgung an der Vielschichtigkeit der Wirklichkeit vorbei, die weit überwiegend nur einer von den Naturwissenschaften geprägten Vorstellung von Gesundheit folgt und einen somatischen Normzustand herbeizuführen bemüht ist.

- Als Reaktion auf das Übermächtigwerden der stark naturwissenschaftlich ausgerichteten Medizin, d. h. einer Medizin, die sich vornehmlich somatische Sachverhalte zum Gegenstand ihres Interesses machte, ist die Forderung nach einer ganzheitlichen Behandlung aufgekommen, ein biopsychosoziales Krankheitskonzept entstanden. Bei der starken Abhängigkeit von Gesundheit von den genetischen, materiellen, individuellen und sozialen Lebensbedingungen ist das sachgerecht. Die Aufzählung der qualitativ verschiedenen Ebenen, auf denen krank machende Bedingungen liegen können und eine Gesundung erfolgen kann, führt aber noch nicht zu einer Theorie vom Rang 
der Humoral- oder Solidarpathologie. So gibt es denn auch bis heute keine Techniken, die sich auf das biopsychosoziale Konzept stützen würden. Die Praxis wird vielmehr beherrscht von der somatischen, ganz überwiegend kurativen Medizin. Auch soweit diese präventive Ziele verfolgt, ist sie - schon wegen der Verfügbarkeit einschlägiger Ansätze - somatisch ausgerichtet, wie das Konzept der Risikofaktoren zeigt. Aus ihnen folgen Handlungsanweisungen, die angesichts der hohen Komplexität der Ätiologie und Pathogenese vor allem der sog. Zivilisationskrankheiten nachgerade primitiv erscheinen.

- Je höher die Komplexität eines Sachverhaltes, um so schwieriger ist es, ihn in die gewünschte, im voraus festgelegte Richtung zu lenken. Es ist, als würde man ein einziges Element eines vielgliedrigen Mobiles anstoßen, um dieses als Ganzes in einen bestimmten Endzustand $\mathrm{zu}$ bringen. Genauso ausgeschlossen ist es, durch die bewußte Gestaltung einiger Lebensumstände und durch eine auf einige Teilziele ausgerichtete Lebensführung die denkbar höchste Gesundheit zu erreichen. Macht man aber mit dem biopsychosozialen Gesundheits- bzw. Krankheitskonzept ernst, dann müßte für alle Lebensbedingungen und die ganze Lebensführung genauso eine materielle Gleichheit realisiert werden wie bei der medizinischen Versorgung.

- Eine materielle Gleichheit bei allen Lebensbedingungen und der gesamten Lebensführung stünde im prinzipiellen Widerspruch zur persönlichen Freiheit, zur Individualität, zum Recht, das Leben entsprechend eigenen Plänen und Präferenzen zu gestalten. Um diese Gestaltung bewerkstelligen zu können, muß nicht nur eine ausreichende Gesundheit als Voraussetzung von Chancengleichheit vorhanden sein, sondern es müssen auch Ressourcen verschiedenster Art eingesetzt werden. Dafür, daß dies richtig geschieht, haftet der einzelne. Eine Ausnahme jedoch macht die medizinische Versorgung. Aufgrund der Kollektivierung des Krankheitsrisikos gibt es die in anderen Wirtschaftsbereichen übliche Einheit von Handlung und Haftung nicht. Sie wurde - zum erstenmal sichtbar zum Ausdruck gebracht durch die Gründung der GKV im Jahre 1883 - aus Zweckmäßigkeitsgründen, nämlich zur Sicherung des sozialen Friedens, der Wirtschaftskraft, der Verteidigungsbereitschaft und des Bevölkerungswachstums, aufgehoben und durch eine Haftung des Kollektivs ersetzt. 
- Diese ursprünglich verfolgten, gleichsam sozialen Ziele verloren im Laufe der Zeit an Bedeutung. Die GKV wurde zu einer auf Umverteilung beruhenden Institution, der die Aufgabe zukam, dem einzelnen immer kompliziertere und aufwendigere medizinische Leistungen ohne expliziten Rückgriff auf begrenzte soziale Ziele zu finanzieren. Die Beibehaltung der Finanzierungs- und Güterverteilungsmodalitäten wurde zum einen durch den Hinweis auf die Kostenträchtigkeit mancher Behandlungen gerechtfertigt, die es den wirtschaftlich Schwachen unmöglich machen würde, Leistungen in Anspruch zu nehmen. Das würde den sozialen Frieden gefährden, weil so die Ungerechtigkeit auf diesem der Kreatürlichkeit des Menschen so nahen Bereich in einer Weise deutlich würde, die uns mehr berühren würde als Ungerechtigkeiten auf anderen Gebieten. Zum anderen wurde die mangelnde Konsumentensouveränität ins Feld geführt und die bei ernster Erkrankung bestehende Notlage des Patienten, die ihn zum wehrlosen Opfer eines Leistungserbringers machen könnte. Auf dieser Vorstellung eines unmündigen Bürgers in erster Linie beruht die aktuelle Zwangskrankenfürsorge: Dem einzelnen soll die Einsicht und moralische Kraft fehlen, für die eigene Zukunft und die Wechselfälle des Lebens zu sorgen. Dabei wird die Wohlfahrt des Staates selbst paradoxerweise der Entscheidung von Wählern überlassen, die der gleiche Staat für unfähig hält, ihre eigenen Angelegenheiten zu regeln.

- Unter völlig veränderten Ausgangsbedingungen ökonomischer, medizinischer, sozialer, demographischer Art sind die genannten Prinzipien der Zwangsfürsorge und der Aufhebung der Einheit von Handlung und Haftung in Frage zu stellen. Völlig verändert haben sich die Finanzierungsvoraussetzungen und die Bedingungen der Leistungsinanspruchnahme, aber auch die ethischen Zielsetzungen und die damit zusammenhängenden sozialen Absichten. An die Stelle einer Zweckmäßigkeits- ist eine Gerechtigkeitsethik, an die Stelle der Gewährleistung einer hohen Bevölkerungsgesundheit ist die Befriedigung individueller Bedürfnisse getreten. Unverändert kann man in der auch damit verbundenen und zur Rechtfertigung des Status quo beschworenen Sicherung des sozialen Friedens ein wichtiges Anliegen der Kollektivierung des Krankheitsrisikos sehen - aber der Nutzen, der für das Gemeinwesen darin liegt, wird zunehmend durch die hohen Kosten aufgehoben, die sich daraus ergeben, daß die Finanzierung der medizinischen Versorgung die 
Erfüllung anderer öffentlicher Aufgaben und den Wirtschaftsstandort Bundesrepublik Deutschland gefährdet. So wie vor mehr als 100 Jahren die Kollektivierung des Krankheitsrisikos im gesellschaftlichen Interesse lag, so ist zu prüfen, ob nicht unter veränderten Bedingungen nun die Reprivatisierung im Interesse der Gemeinschaft liegt.

Es ist dies nicht von heute auf morgen zu bewerkstelligen. Daher kann es im folgenden beim Aufzeigen von Entwicklungsperspektiven des Versorgungssystems auch nicht darum gehen, ein übergangslos zu realisierendes neues Konzept vorzustellen, sondern die Schritte aufzuzeigen, die, von einer Konsolidierung und Verbesserung des Status quo ausgehend, allmählich zu seiner Überwindung führen können.

\section{Die Versorgung im Rahmen der GKV}

\section{Einnahmenseite}

Vor allem im Zusammenhang mit dem GRG von 1989, aber auch noch danach wurde ungeachtet zahlreicher teilweise radikaler Reformvorschläge, die vor allem zum Ziel hatten, eine höhere Effizienz durch Beseitigung von Fehlsteuerungen zu erreichen, weit überwiegend von der "Bewährtheit“ der GKV gesprochen. Angesichts des Funktionswandels, den die GKV seit ihrer Gründung erfahren hat und der auch scheinbar tragende Prinzipien wie Solidarität betrifft, ist eine solche Aussage schon vom Begrifflichen her problematisch: Das Urteil bezieht sich heute im letzten auf etwas in qualitativer und quantitativer Hinsicht anderes als das, was vor 100 oder 50 Jahren bestand. Problematisch ist es aber auch vom Sachlichen her, wenn man die ständigen Interventionen bedenkt, die zum Erhalt des Systems erforderlich waren und dann noch die Schwierigkeiten berücksichtigt, denen sich das System derzeit gegenüber sieht.

Richtig ist hingegen, daß mit Hilfe des GKV-Systems am Anfang gewisse soziale Ziele verfolgt werden konnten und daß in den Zeiten starken wirtschaftlichen Wachstums nach dem Weltkrieg mit Hilfe der GKV eine Wertschöpfung und eine Umverteilung gelang, die es erlaubten, ein im Hinblick auf die Breite der Ansprüche und die realisierte Versorgungsgerechtigkeit beispielhaftes Versorgungssystem aufzubauen und zu finanzieren. 
In diesem Erfolg liegen aber auch einige Gründe der heutigen Probleme, wie z. B. Überkapazitäten und überbordende Ansprüche. Mit der Veränderung der ökonomischen Rahmenbedingungen - verlangsamtes Wirtschaftswachstum und Abnahme der für die Beitragsschöpfung entscheidenden Lohnquote - mußte zwangsläufig das System dekompensieren.

Dieses System war auch in Zeiten seiner optimalen Funktionsfähigkeit nicht rational zu begründen, denn man benötigte keine ca. 1200 Kassen - die es vor 10 Jahren noch gegeben hat -, um zu unterschiedlichen Beitragssätzen ein im großen und ganzen einheitliches Leistungspaket zu finanzieren. Das System ist schon lange nur historisch zu verstehen, was ein durchaus respektabler Legitimationsgrund sein kann. Es ist aber die Frage, was man es sich kosten lassen will, ein solches System auf Biegen und Brechen zu erhalten, z. B. mit einem aufwendigen Risikostrukturausgleich, der es in Frage stellt, ob man noch von gegeneinander abgrenzbaren Solidargemeinschaften sprechen kann, die doch sinnhaft nur durch eine beschränkte regionale oder aber durch Beruf oder Beschäftigungsstatus bestimmte Reichweite gegeben sein können. Diese Abgrenzungen sind mit dem Einräumen von Wahlfreiheit aufgehoben worden. Da stellt sich die Frage, ob man nicht, statt weiterhin mühsame Korrekturen vorzunehmen, gleich zu einer Steuerfinanzierung übergehen sollte. Dies um so mehr, als damit eine Reihe weiterer Mängel beseitigt werden könnte, deren Abstellung immer dringender gefordert wird:

- Die Abhängigkeit der Höhe des Beitragsaufkommens vom Faktor Arbeit und die Belastung der Lohnnebenkosten

- Die Problematik versicherungsfremder Leistungen

- Die Problematik wachsender Defizite in den neuen Bundesländern durch die dortige Langzeitarbeitslosigkeit

- Die Problematik unterschiedlicher Risiken (auf der Ausgabenseite)

- Den kontraproduktiven Wettbewerb zwischen den Kassen

- Das Bestehen von Beitragsbemessungsgrenzen.

Eine Reihe von aktuell diskutierten Reformvorschlägen zielt auf eine Aufhebung der Beitragsbemessungsgrenzen und die Erweiterung der Bemessungsgrundlage. Damit nähert sich das System tendenziell bereits einem steuerfinanzierten, und es bedarf dann mit Sicherheit weiterer komplizierter Regeln, um an der Fiktion eines bewährten beitragsfinanzierten Systems festzuhalten. 
Ein steuerfinanziertes System gibt dem Staat größere Gestaltungsmöglichkeiten bei den leistungsbestimmenden Kapazitäten, Strukturen und Anreizen. Bei einer 100\%igen Steuerfinanzierung fehlt andererseits der ja nicht nur kontraproduktive, sondern durchaus auch leistungssteigernde Wettbewerb zwischen selbständigen Kassen. Dabei ist aber kein steuerfinanziertes System denkbar, das unbeschränkt Leistungen bereitstellen könnte. Die Nachfrage nach Gütern, die kostenlos abgegeben werden, wird immer größer sein als das Angebot, so daß Engpässe in Form von Wartelisten und Wartezeiten unvermeidbar sind. Ebenso unvermeidlich wird sich unter diesen Umständen ein privat finanzierter Nebenmarkt auftun, um die Engpässe zu überwinden und eine größere Freiheit zu haben, die im offiziellen System im Interesse einer sparsamen Mittelverwendung eingeschränkt werden muß.

Das dann gegebene Nebeneinander eines steuer- und eines prämienfinanzierten Systems fördert den Wettbewerb, aber auch den Aufbau von Strukturen, um im Sinne einer Reprivatisierung von Krankheit im Laufe der Zeit und über die Definition einer Grundsicherung von der Kollektivierung des Krankheitsrisikos wegzukommen.

\section{Ausgabenseite}

Es ist von den Konsequenzen her gesehen bedeutungslos, ob die Schere zwischen dem Finanzierbaren und dem Wünsch- bzw. Machbaren sich durch Entwicklungen auf der Einnahmen- oder auf der Ausgabenseite öffnet. Die seit Jahren von vielen Sachkennern festgestellten Einsparpotentiale aber bestehen nur auf der Ausgabenseite, und folgerichtig muß dort mit Maßnahmen zur Effizienzsteigerung angesetzt werden.

Die Feststellung, daß es im medizinischen Versorgungssystem Rationalisierungsreserven gibt, ist trivial. Es gibt sie in allen Wirtschaftsbereichen, und sie sind völlig unvermeidlich in einem System, in dem die Güter und Leistungen zum Nulltarif nach Bedürftigkeit verteilt werden und diejenigen, die über das Vorliegen eines Bedürfnisses entscheiden, durch seine Befriedigung ihr Einkommen erhöhen können. Bei diesem Bemühen sind ihnen durch die Definition der Leistungsanrechte grundsätzlich Grenzen gesetzt, die, würden sie strikt eingehalten, es vermutlich erlauben würden, den vornehmlich durch die demographische Entwicklung und den medizinischen Fortschritt bedingten Ausgabenanstieg im 
Rahmen des Anstiegs der beitragspflichtigen Einnahmen der Versicherung zu halten. Die Grenzen sind mit dem Gebot der Wirtschaftlichkeit in $\S 12$ SGB V gezogen. Nach Satz 1 hat der Versicherte Anrecht auf eine ausreichende, zweckmäßige, wirtschaftliche und das Maß des Notwendigen nicht überschreitende Behandlung.

So klar und überzeugend es auf Anhieb erscheint, derart einsichtige Kriterien für die Leistungen vorzugeben, die für eine Behandlung im o. g. Sinne erforderlich sind: Es ist mit ihrer Hilfe nicht abzuleiten, was dies inhaltlich und für den Einzelfall bedeutet. Das Leistungsgeschehen als Ganzes ergibt sich nicht aus den bei einer abstrakten Sicht der Dinge bestimmenden Faktoren "Krankheitslast" und "Stand der medizinischen Wissenschaft", und es hängt im Einzelfall, vor allem wenn verschiedene Behandlungsoptionen vorliegen, vom dispositionellen Wissen des Leistungserbringers $a b$, aber auch von den Erwartungen und Wünschen des Patienten. Eine Rolle spielen sowohl auf der Systemebene als auch auf der von Arzt und Patient weiterhin die Strukturen, Kapazitäten und Anreize eines Versorgungssystems.

Besonders schwer wiegt in diesem Zusammenhang, daß für viele Verfahren und Leistungen gar nicht in einer intersubjektiv nachprüfbaren Weise bekannt ist, ob sie zweckmäßig, d. h. effektiv und weitergehend, ob sie wirtschaftlich, d. h. kosteneffektiv, sind und welche Leistungsmenge ausreichend ist. Könnte dies gewährleistet werden, wäre die höchst denkbare Effizienz erreicht. Die Frage ist, mit welchen Ansätzen dieses Ziel wenigstens annäherungsweise verwirklicht werden könnte.

\section{Ansätze zur Einlösung des Wirtschaftlichkeitsgebotes als kurzfristiger Perspektive}

\section{a) Zweckmäßigkeit}

Der Nachweis der Effektivität ist speziell bei Arzneimitteln und einer Reihe von Medizinalprodukten als Voraussetzung für die $\mathrm{Zu}$ lassung und im großen und ganzen auch für die Kostenerstattung im Rahmen der gesetzlichen Krankenversicherung üblich. Bei allen anderen diagnostischen und therapeutischen Verfahren ist das nur ausnahmsweise der Fall. Für die Zulassung bei Geräten reicht im allgemeinen die technische Sicherheit aus, bei Behandlungsstrategien, z. B. bestimmten Schemata der Chemotherapie 
von bösartigen Tumoren oder für chirurgische Techniken, gibt es keine formalisierte Zulassung.

Die strikte Übertragung der bei Arzneimitteln verwendeten Prüfverfahren auf andere Behandlungen scheitert am finanziellen, zeitlichen, methodischen und personellen Aufwand, ganz abgesehen von den Schwierigkeiten, für bestimmte Indikationen eine ausreichende Patientenzahl zusammenzubringen.

Besonders bei kapital- und personalaufwendigen Großgeräten, aber wegen ihrer Kostenrelevanz zunehmend auch bei sog. small-ticket-Technologien, wird eine Technikfolgeabschätzung und Nutzenevaluation gefordert. Deutschland ist Entwicklungsland auf diesem Gebiet, doch wäre zu prüfen, ob andere Länder mit teilweise hochspezialisierten Institutionen zur Durchführung entsprechender Untersuchungen und großem Personalbestand, denen diese Aufgabe obliegt, besser dastehen, d. h. ein rationelleres Leistungsgeschehen durch die Konzentration auf effektive Verfahren aufweisen als die Bundesrepublik Deutschland. Was vielleicht kontrollierter verläuft, ist die Diffusion neuer Verfahren in den ärztlichen Alltag. Dies wird bei uns durch gewisse strukturelle Besonderheiten des Systems - insbesondere die scharfe Grenze zwischen dem vertragsärztlich ambulanten und dem stationären Bereich sowie das Nebeneinander von sozialer und privater Krankenversicherung - erleichtert.

Über die Größenordnung der mit dem Einsatz ineffizienter, d. $h$. nicht zweckmäßiger Verfahren verbundenen Probleme und die von ihrer Elimination erhofften Einsparungen sind kaum valide Aussagen möglich. Die auffallende Stereotypie, mit der in der gesundheitspolitischen und gesundheitsökonomischen Diskussion als Beispiel für eine weitgehend überflüssige Methode die Osteodensitometrie angeführt wird, läßt vermuten, daß auf Anhieb jedenfalls nicht so viele Verfahren genannt werden können, um durch ihren Ausschluß aus dem Leistungskatalog die wie auch immer, d. h. auf der Einnahmen- oder Ausgabenseite begründeten Defizite der GKV zu vermeiden oder sie auch nur nennenswert zu verringern. Der SVR geht in seinem Sondergutachten bei der Osteodensitometrie von Einsparungen in Höhe von ca. 100 Mio. DM aus. Um das Defizit der GKV des letzten Jahres in Höhe von 6,3 Mrd. DM aufzufangen, müßten 63 Verfahren mit vergleichbarer Kostenrelevanz identifiziert und eliminiert werden. 
Vor dieser Modellüberlegung ist die vom Bundesausschuß der Ärzte und Krankenkassen mit dem 2. NOG in § 135 SGB V neugefaßte Aufgabe zu sehen, den diagnostischen und therapeutischen Nutzen neuer, vor allem aber auch bereits angewandter Methoden sowie deren medizinische Notwendigkeit und Wirtschaftlichkeit zu überprüfen. Es setzt dies nicht nur einen ausreichend großen Apparat von Fachleuten voraus, die die in ihren Ergebnissen ja keineswegs übereinstimmenden Untersuchungen aus aller Herren Länder auswerten müssen, sondern auch einen langen Atem, um die rechtlichen und berufspolitischen Probleme zu lösen, die bei dem Ausschluß eines alten Verfahrens oder der Nichtzulassung eines nur marginal verbesserten neuen zu erwarten sind. Auf keinen Fall ist dies in der Zeit zu leisten, die uns verbleibt, um angesichts einer zunehmenden Öffnung der Schere zwischen dem technisch Machbaren und dem nach Expertenmeinung Wünschbaren auf der einen Seite und dem Finanzierbaren auf der anderen Seite Einsparungen zu erzielen.

\section{b) Notwendigkeit}

Die Probleme im Versorgungssystem rühren weniger von dem Einsatz nichteffektiver Verfahren her, sie hängen vielmehr entscheidend mit der Anwendung grundsätzlich effektiver Verfahren bei nicht gegebener Indikation zusammen. Selbst auf die Osteodensitometrie möchte man ja nicht völlig verzichten, sowenig wie auf das Langzeit-EKG, die Langzeitblutdruckmessung oder das EEG. Die entsprechenden Untersuchungen sollen nur nicht so oft durchgeführt werden, wie es der Fall ist.

Daß sie so oft durchgeführt werden, hat eine Reihe von Gründen, z. B. hängt es mit dem Bemühen des Arztes zusammen, den im Laufe der Berufsausübung internalisierten Standard eines Systems zu erreichen. Weiterhin gibt es forensische Rücksichten, Auswirkungen des Kassenwettbewerbs, Patientenwünsche und schließlich das einzelwirtschaftlich rationale Bemühen, durch eine Mengenausweitung das Einkommen zu maximieren. Das ist ein besonders leicht verständlicher kontraproduktiver Effekt, der sich unter den Bedingungen der gedeckelten Gesamtvergütung einstellt.

Auf die Bedeutung der nichtmedizinischen Bestimmungsfaktoren für das Leistungsgeschehen wurde schon hingewiesen, nachzutragen ist, daß es keine Theorie gibt, mit deren Hilfe die nach Art 
und Menge angemessenen Leistungen zu berechnen wären. Da nun den nichtmedizinischen Bestimmungsfaktoren ein so großes Gewicht zukommt, ist es vernünftig (und allein erfolgversprechend), dort zur Änderung der Verhältnisse anzusetzen. Strukturen, Kapazitäten und Anreize sind also so zu gestalten, daß das Wünschenswerte erreicht wird. Im einzelnen können dazu zählen:

- Die Einrichtung eines Primärarztsystems. Der Haus- oder Primärarzt kanalisiert den Zugang zu aufwendigeren Versorgungsstufen und wirkt überhaupt als "Fallmanager".

- Die Beschränkung der Kapazitäten von Spezialisten, Betten und Großgeräten bis zur Knappheitsgrenze, die dann erreicht ist, wenn Wartezeiten bestehen und merkliche Engpässe auftreten.

- Die Beseitigung von Anreizen, die nach aller Erfahrung einer Mengenausweitung förderlich sind, also die Einzelleistungsvergütung, die durch eine weitgehende Pauschalvergütung zu ersetzen ist.

- Die Anwendung von Richtlinien bzw. Behandlungsprotokollen, mit deren Hilfe die Indikation zu aufwendigen Behandlungen im Einzelfall zu überprüfen ist.

Richtlinien und Behandlungsprotokolle können nur dann zu einer Leistungsreduktion führen, wenn ihre Einhaltung kontrolliert wird und ihre Nichteinhaltung Sanktionen nach sich zieht. Im Rahmen von Managed Care sind in den USA eine Reihe von Instrumenten entwickelt worden, um das zu erreichen, worauf hier nicht weiter eingegangen werden kann.

\section{c) Wirtschaftlichkeit}

Die Änderung von Kapazitäten, Strukturen und Anreizen erfolgt mit der Absicht, die Leistungen auf das Notwendige zurückzuführen und so Einsparungen zu erzielen. Das ist - sofern an dem sozialpolitischen Ziel festgehalten wird, in kollektiver Anstrengung Gesundheit als Voraussetzung zur Herstellung von Chancengleichheit bei der Verwirklichung von Lebensplänen zu gewährleisten - nur zu rechtfertigen, wenn damit keine Nachteile bei der Verfolgung der Zwecke der Medizin verbunden sind. Der internationale Vergleich zeigt, daß diese Befürchtung in weitem Umfang grundlos ist und die von Ärzten gerne geglaubte Beziehung, mehr 
Geld $=$ mehr Leistungen $=$ mehr Gesundheit, nicht besteht. Die Konzentration des Leistungsgeschehens auf das Zweckmäßige und Notwendige erhöht aber per se die Wirtschaftlichkeit der Versorgung, die durch eine Bevorzugung von kosteneffektiven Verfahren noch weiter erhöht werden kann.

Managed-Care-Organisationen setzen sich neben einer Verbesserung der Qualität explizit zum Ziel, Einsparungen zu erreichen. Über eine kostengünstige Gestaltung der nichtmedizinischen Bestimmungsfaktoren hinaus kann dies durch die Bevorzugung preiswerter Leistungen gelingen. Ein überzeugendes Beispiel ist die Verwendung billiger Generika anstelle teurer Originalpräparate. Bei anderen Behandlungen liegen die Verhältnisse nicht ganz so einfach, weil es oft keine gleichwertigen preisgünstigeren Alternativen gibt. Es ist aber oft möglich, im Rahmen einer Regelversorgung auf die Vergütung teurer, in ihrer Wirkung umstrittener Behandlungen zu verzichten, ohne daß damit meßbare Nachteile für den Patienten verbunden wären. So ist im allgemeinen bei einem Herzinfarkt kein zusätzlicher Nutzen durch eine PTCA nach einer Lyse zu erwarten, und die Streßechokardiographie bringt keine therapierelevanten Mehrinformationen gegenüber einer normalen Echokardiographie.

In vergleichbarer Weise können Verfahren mit geringem Grenznutzen im Hinblick auf die mit ihrer Anwendung verbundenen hohen sozialen Opportunitätskosten aus dem Leistungskatalog ausgeschlossen werden. Ein Beispiel ist die durchgängige Anwendung von t-PA anstelle von Streptokinase. Von 100 Patienten profitiert nur einer davon, und auf ihn sind die dadurch verursachten Mehrkosten zu beziehen. Die Akzeptanz solcher Strategien zur Kostenbegrenzung wird verbessert, wenn dem Arzt grundsätzlich die Möglichkeit eingeräumt wird, in begründeten Fällen von der Regel abzuweichen. Aber dies ist wiederum nur vertretbar, wenn durch Kontroll- und Sanktionsmechanismen ein Mißbrauch verhindert wird. Hier zeigt sich aber auch die Kehrseite von mehr Regulation und Kontrolle: Es ist damit ein Anstieg der Verwaltungskosten verbunden, der leicht die Höhe der erzielten Einsparungen übertreffen kann.

\section{d) Fazit}

Auf Anhieb überzeugt die Bestimmung des $\S 12$ (1) SGB V, daß der Versicherte nur ein Anrecht auf ausreichende, zweckmäßige und wirtschaftliche Leistungen hat. Eine genauere Betrachtung 
zeigt jedoch, daß sich aus diesen Kriterien nicht das dem Einzelfall Angemessene ableiten läßt. Und weiterhin, daß es große Schwierigkeiten bereitet, die entsprechenden Verfahren auszumachen und das Leistungsgeschehen auf das zu beschränken, was diesen Kriterien genügt.

Auch die mit dem zweiten NOG vorgesehene Überprüfung der Behandlungsverfahren durch den Bundesausschuß der Ärzte und Krankenkassen ( $\S 135$ SGB V) wird kaum dazu beitragen, die Finanzierungsprobleme im Versorgungssystem zu lösen. Angesichts solcher Perspektiven ist die Frage, ob nicht an den tragenden Prinzipien der medizinischen Versorgung in den Wohlfahrtsstaaten angesetzt werden muß, um eine wirkliche Problemlösung zu erreichen. 


\section{Stärkung von Eigenverantwortung als langfristige Perspektive}

\section{a) Allgemeines}

Die tragenden Prinzipien des heutigen Versorgungssystems sind die Entprivatisierung von Krankheit, die Kollektivierung des Krankheitsrisikos und die Gewährleistung von materieller Gleichheit bei der Bedürfnisbefriedigung. In die Richtung einer Ablösung dieser Prinzipien zielt die in letzter Zeit immer nachdrücklicher erhobene Forderung nach einer Stärkung von Eigenverantwortung.

Unter Rückgriff auf das Risikofaktorenkonzept zur Erklärung des Entstehens der heute in der Mortalität führenden Krankheiten wird von manchen Protagonisten von einer Eigenverantwortung im spezifisch-medizinischen Sinne ausgegangen.

Der einzelne wäre demnach für Folgen eines Übergewichts, von Bewegungsmangel und Genußmittelmißbrauch haftbar zu machen. Angesichts der Lücken-, ja Zweifelhaftigkeit der Erkenntnisse über die Ätiologie und Pathogenese der sog. Zivilisationskrankheiten ist das nicht zu vertreten.

Wird die Stärkung von Eigenverantwortung als tragender Ansatz zur Verbesserung der Effizienz der Versorgung und einer Ausgabendämpfung gesehen, so heißt das unter Berücksichtigung der Fragwürdigkeit, sie in spezifisch-medizinischer Hinsicht zu definieren, daß damit in allgemeiner Form - also nicht auf bestimmte Krankheitsarten oder Behandlungsindikationen beschränkt - im medizinischen Versorgungssystem ansatzweise jene Einheit von Handlung und Haftung hergestellt wird, wie sie in allen anderen Wirtschaftsbereichen besteht. Nicht für eine bestimmte Krankheit als Folge einer der Gesundheit abträglichen Lebensführung würde der Versicherte haften, sondern für die Inanspruchnahme von Dienstleistungen und -gütern als solche in die ökonomische Verantwortung gezogen. Genau dies wird durch die seit 01.01.1997 gültigen Zuzahlungsregeln erreicht. Über Härtefallregeln sollen zugleich Überforderungen verhindert und soll die Sozialverträglichkeit gewährleistet werden.

Für einen möglicherweise sogar großen Teil der Versicherten mögen die Härtefallregeln irgendwann greifen, doch dürfte von den neuen Bedingungen dennoch eine Steuerungswirkung aus- 
gehen: Die Ansprüche der Bevölkerung sind im Zuge und als Folge der zivilisatorischen und sozialen Entwicklung immer differenzierter geworden und richten sich jetzt auch auf Leistungen und Güter, die ohne Aufgabe des eigentlichen Ziels der solidarisch finanzierten medizinischen Versorgung aufgrund ihrer Natur oder der Höhe ihrer Kosten dem Privatkonsum zugerechnet werden können.

\section{b) Subsidiarität und Solidarität}

Ein weiterer und auch beabsichtigter Effekt der neuen Zuzahlungsregeln wird sein, daß das für die medizinische Versorgung verfügbare Finanzvolumen ohne expliziten äußeren Zwang vergrößert und ohne Auswirkungen auf die Lohnnebenkosten bleiben wird. Es hat jedoch Folgen für den Privatkonsum der Versicherten: Gesundheitsgüter konkurrieren stärker als bisher mit anderen Verwendungszwecken und werden von den Versicherten eine Nutzenabwägung erzwingen. Im Hinblick auf die Wahrscheinlichkeit, zu irgendeinem Zeitpunkt durch eine als medizinisch notwendig eingeschätzte Inanspruchnahme stärker finanziell belastet zu werden, kann es sinnvoll sein, sich zusätzlich abzusichern oder für diesen Zweck Reserven zu bilden. Damit würde die Verantwortung für die Absicherung von Lebensrisiken auf den einzelnen zurückverwiesen, und die Solidargemeinschaft müßte nur in den Fällen in Anspruch genommen werden, in denen die Grenze der eigenen Leistungsfähigkeit überschritten wird. Dies wäre der Einstieg in eine wünschenswerte Neuordnung des Verhältnisses von Subsidiarität und Solidarität, nicht hingegen, um einige wenige "Luxusleistungen" wie 1.-Klasse-Zimmer im Krankenhaus und Kuren, d. h. ganz offensichtliche Präferenzleistungen gesondert abzusichern.

Die Grenze zwischen Subsidiarität und Solidarität würde sich am Kriterium der wirtschaftlichen Leistungsfähigkeit des einzeinen orientieren und nicht an dem, was nach dem Urteil der Leistungserbringer als medizinisch notwendig eingeschätzt wird. Das letztere ist für den weitaus größten Teil des Leistungsgeschehens nicht mit der wünschenswerten Sicherheit zu definieren, wie vor allem die teilweise dramatischen Unterschiede bei den Mengen bestimmter Leistungen zwischen verschiedenen Systemen, aber auch innerhalb eines Systems zwischen verschiedenen Regionen zeigen. Was angemessen, $d . h$. was medizinisch notwendig ist, kann im Grunde niemand sagen, weil es nicht möglich ist, einen 
Bedarf zu objektivieren oder inn aus der (unbekannten) Krankheitslast unter Berücksichtigung des Standes der medizinischen Wissenschaft zu berechnen. Das gilt $u$. a. für die Zahl der Ärzte, von Großgeräten, Krankenhausbetten, Pflegekräften, Arzneimitteln. Eine gesetzmäßige Abhängigkeit zwischen der Menge medizinischer Leistungen und der öffentlichen Gesundheit fehlt, gemessen am harten Indikator Lebenserwartung.

\section{c) Fazit}

Der Verzicht auf die Stärkung der Eigenverantwortung durch eine Neuordnung der Verhältnisse von Subsidiarität und Solidarität im vorgenannten Sinne wird meist durch Rückgriff auf gleichsam emotionale Argumente, z. B. durch den Hinweis auf die Hilflosigkeit des Kranken, gerechtfertigt. Rational betrachtet, fällt es aber schwer, sich einer so grundsätzlichen Lösung zu entziehen, denn mit den genannten Ansätzen soll ja gerade erreicht werden, daß auch in Zukunft für die Versorgung der ernsthaft kranken und unstrittig bedürftigen Patienten ausreichend Mittel zur Verfügung stehen.

Wer dies nicht so sieht, vielmehr am Status quo, und zwar unter der Bedingung politisch verordneter Ausgabenbeschränkung festhalten will, muß erklären, wie die dann unvermeidliche Rationierung von medizinischen Leistungen erfolgen soll. Es ist ein Irrtum zu glauben, daß man den Widerspruch zwischen dem medizinisch Machbaren und dem kollektiv Finanzierbaren in einer harmonischen Weise, d. h. ohne merkliche soziale und individuelle Kosten lösen könnte. Wenn wir wollen, daß auch künftig eine Chance besteht, alles wirklich Wünschbare in der Medizin zu gewährleisten, dann - so wäre frei nach Lampedusa zu sagen müssen wir das Gewohnte ändern. Dies aber könnte durch die aktuelle "Begriffsoffensive" ein Stück weit gefördert werden.

\section{Ausblick}

Der Absicht des 2. Bad Orber Gespräches entsprechend, kontroverse Themen aufzugreifen sowie mit diesem Vortrag Ausgangspunkte für die Diskussion in den Arbeitsgruppen zu liefern, wurden vorstehend Entwicklungsmöglichkeiten der medizinischen Versorgung sowohl auf der Einnahmen- als auch der Ausgabenseite beschrieben. Sie reichen von relativ einfachen, gleichsam technischen Maßnahmen wie der Reform der Vergütungsmodali- 
täten bis zur radikalen Neuordnung der ökonomischen Verantwortung des einzelnen für Krankheit. Wäre der Status quo fehlerfrei und für alle zufriedenstellend, dann käme niemand auf die Idee, das System teilweise grundlegend zu ändern. Aber das ist nicht der Fall, vielmehr gibt es, wie bekannt und hier noch einmal angedeutet, Probleme auf der Einnahmenseite und einen offenbar auf keine Weise dauerhaft zu beherrschenden Ausgabenanstieg. Wir sind gefordert, hierfür Lösungen zu finden.

In der letzten Sitzung des Bundesausschusses der Ärzte und Krankenkassen am 01.10.1997 hat Minister Seehofer einige Feststellungen getroffen, die in weitgehender Übereinstimmung mit den vorstehend abgeleiteten Entwicklungsperspektiven stehen:

- Die Beitragsschöpfung muß im gesamtwirtschaftlichen Interesse von der Arbeit abgekoppelt werden.

- Es gibt große Rationalisierungsreserven im System, die mit allen Mitteln mobilisiert werden sollten.

- Das Leistungspaket ist um ineffiziente Verfahren zu bereinigen.

- Auch bei Mobilisierung von Wirtschaftlichkeitsreserven und einer Bereinigung des Leistungspaketes wird es erforderlich sein, die Mittel für die medizinische Versorgung zu erhöhen.

- Es ist zu prüfen, ob nicht an die Stelle einer Pflichtversicherung eine Versicherungspflicht treten sollte.

Gegen die Realisierung dieser Forderungen gibt es vielfältige, schon aus der durch die Größe des Versorgungssystems bedingten Trägheit folgende Widerstände. Sie hängen maßgeblich mit dem Bemühen der verschiedenen Wirtschaftssubjekte zusammen, die erworbenen Besitzstände zu wahren. Diesem Vortrag ist als Motto eine Passage aus dem 7. Buch der "Geschichte der Stadt Rom im Mittelalter" von Gregorovius vorangestellt, das diesen Sachverhalt in allgemeiner Form auf den Punkt bringt. Wir müssen uns damit abfinden, daß die darin erwähnten Kräfte es sind, die die soziale Wirklichkeit gestalten. Aber auch der wissenschaftliche Diskurs, dem die Bad Orber Gespräche dienen, ist eine solche Kraft, selbst wenn er nur langfristig und in Bruchstükken zum Tragen kommen kann. 
Herbert Rebscher, Eva Walzik

\section{Aktuelle Finanzlage der GKV}

Die gesetzlichen Krankenkassen verzeichneten im ersten Halbjahr 1997 ein Defizit von ca. 4 Mrd. DM. Während die Entwicklung der Leistungsausgaben moderat verlief, stagnierten bzw. sanken die beitragspflichtigen Einnahmen der Versicherten (Übersicht 1). Die deutlichen Einsparungen durch die Anstrengungen der Selbstverwaltung sowie die problematischen Auswirkungen des Beitragsentlastungsgesetzes mit Leistungskürzungen und Zuzahlungserhöhungen zeigen sich vor allem bei den Ausgabenrückgängen in den Bereichen Heil- und Hilfsmittel $(-3,5 \%)$, Krankengeld $(-20,2 \%)$, Kuren $(-19,6 \%)$ und Soziale Dienste/Gesundheitsförderung $(-35,1 \%)$.

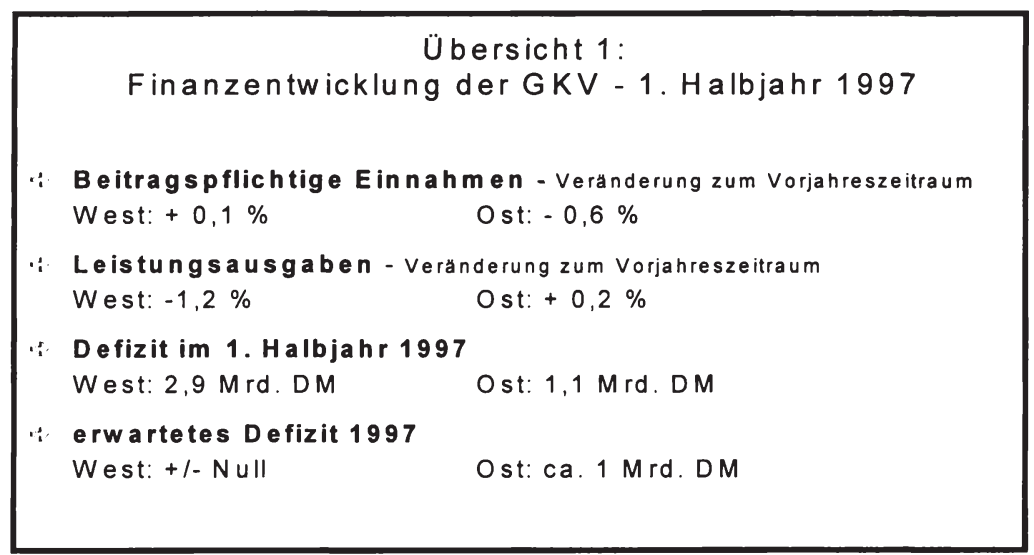

Hohe Arbeitslosigkeit, Abbau von Überstunden, Kürzungen beim Urlaubsgeld im Rahmen der Sicherung der Lohnfortzahlung im Krankheitsfall, zunehmende untertarifliche Bezahlung, Zunahme der nicht versicherungspflichtigen 610/520-Mark-Jobs sowie geringe Rentensteigerungen entziehen der gesetzlichen Krankenversicherung (GKV), insbesondere in den neuen Bundesländern, zunehmend die Einnahmenbasis.

Einschließlich des Defizits des vergangenen Jahres summiert sich der Finanzierungssaldo der GKV-Ost inzwischen auf 
2,3 Mrd. DM. Eine Deckung dieses Defizits würde eine Beitragssatzerhöhung von 0,77 Beitragssatzpunkten erfordern, was bei einem durchschnittlichen Beitragssatz der GKV-Ost von derzeit $13,95 \%$ (August 1997) als nicht mehr akzeptabel erscheint. Um Beitragssatzanhebungen zu vermeiden, finanziert sich inzwischen eine Reihe von Krankenkassen in den neuen Bundesländern über Kredite. Sparmaßnahmen der Krankenkassen allein werden nicht mehr ausreichen, um eine finanzielle Konsolidierung der GKV-Ost zu erreichen.

Im Oktober 1997 wurde deshalb auf Initiative des Bundesgesundheitsministers mit den zuständigen Ost-Länderministern sowie Vertretern der Spitzenverbände der gesetzlichen Krankenkassen ein Lösungsvorschlag zur Rechtsangleichung in der GKV erarbeitet, der schnelle und zielgerichtete Hilfe für die Krankenkassen in den neuen Bundesländern verspricht. Der Lösungsvorschlag beinhaltet drei Grundelemente:

- sofort einsetzende strikte Sparmaßnahmen,

- gezielte kassen- bzw. kassenarteninterne Selbsthilfe und

- Einführung eines zunächst auf den Grundlohnausgleich beschränkten gesamtdeutschen Risikostrukturausgleichs ab 1999.

Falls es gelingt, für diesen Vorschlag einen partei- und länderübergreifenden Konsens zu finden, könnte noch in diesem Jahr das entsprechende Gesetzgebungsverfahren eingeleitet, zum Abschluß gebracht und damit ein entscheidender Beitrag zur Angleichung der Lebensverhältnisse von Ost und West geleistet werden.

\section{Ursachen der Finanzprobleme}

Aufgrund der wirtschaftlichen Entwicklung ist zu erwarten, daß die Einnahmenschwäche der Krankenkassen zumindest mittelfristig bestehenbleiben wird. Dies gilt, wenn auch in unterschiedlichem Ausmaß, für die neuen wie die alten Bundesländer. Damit rücken zunehmend Reformvorschläge in den Vordergrund, die auf der Einnahmenseite ansetzen.

Die Diskussion von mittelfristigen Strategien zur Stabilisierung der Einnahmenseite darf allerdings nicht den Blick dafür verstellen, daß dieses System nach wie vor ausgabenseitige Probleme in 
enormem Ausmaß hat. So schlummern Rationalisierungspotentiale in der Struktur des medizinischen Angebots, und eine Problemlösungsstrategie muß deshalb zuvorderst eine Rationalisierungsstrategie im Rahmen einer solidarischen Wettbewerbsordnung sein.

Gerade die aktuelle Finanzsituation der Ost-Krankenkassen zeigt nämlich die Fragwürdigkeit einseitiger Begründungen. Die Ursachen liegen eben nicht nur auf der Einnahmenseite. Die hohen Steigerungsraten bei Zahnersatz sowie das hohe Pro-KopfNiveau bei Arzneimitteln, das bereits über dem in den alten Bundesländern liegt, oder das immer noch ungelöste Krankenhausproblem zeigen den Handlungsbedarf auch auf der Ausgabenseite. Mit wechselnder Gewichtung gab es in der GKV bereits in der Vergangenheit immer einnahmen- und ausgabenseitig bedingte Probleme (Übersicht 2 und 3). Aus diesem Grund werden die Diskussionen über die Finanzierungsprobleme der GKV in den Ersatzkassen nicht unabhängig von Strukturveränderungen zur Ausgabenbegrenzung geführt.

\section{Übersicht 2: \\ Ursachen der GKV-Finanzprobleme}

\section{Einnahmenseite}

- hohe Arbeitslosigkeit

- Abbau von Überstunden

- Kürzungen beim Urlaubsgeld

- zunehmende untertarifliche

Bezahlung

- geringe Rentenerhöhungen

- Zunahme der 610-Mark-Jobs

\section{Ausgabenseite}

- Veränderung der Morbidität

- demographische Entwicklung

- medizinisch-technischer Fortsch

- Überkapazitäten

- verändertes Nachfrageverhalter

- strukturelle Probleme, wie z.B. fehlende Verzahnung

- "Verschiebebahnhöfe" der Politik etc. 
Übersicht 3:

GKV - Ausgaben und Beitragseinnahmen

je Mitglied in DM und Veränderung zum Vorjahr in Prozent - 1987 bis 1996

Alte Bundesländer

$\begin{array}{lrrrrrr}\text { Jahr } & \text { Ausgaben } & \begin{array}{c}\text { Veränd. } \\ \text { in } \%\end{array} & \begin{array}{c}\text { Beitragspflichtige } \\ \text { Einnahmen }\end{array} & \begin{array}{c}\text { Veränd. } \\ \text { in } \%\end{array} & \begin{array}{c}\text { Beitrags- } \\ \text { einnahmen }\end{array} & \begin{array}{c}\text { Veränd. } \\ \text { in } \%\end{array} \\ 1987 & 3.404 & 3,5 & 31.759 & 2,2 & 3.292 & 5,3 \\ 1988 & 3.632 & 6,7 & 32.827 & 3,4 & 3.464 & 5,2 \\ 1989 & 3.490 & -3,9 & 33.979 & 3,5 & 3.623 & 4,6 \\ 1990 & 3.734 & 7,0 & 35.704 & 5,1 & 3.739 & 3,2 \\ 1991 & 4.106 & 10,0 & 37.506 & 5,0 & 3.807 & 1,8 \\ 1992 & 4.507 & 9,8 & 39.392 & 5,0 & 4.111 & 8,0 \\ 1993 & 4.444 & -1,4 & 40.957 & 4,0 & 4.511 & 9,7 \\ 1994 & 4.847 & 9,3 & 41.810 & 2,4 & 4.657 & 3,4 \\ 1995 & 5.368 & 10,7 & 42.101 & 0,7 & 4.666 & 0,2 \\ 1996 & 5.463 & 3,1 & 42.554 & 1,1 & 4.798 & 3,1\end{array}$

Neue Bundesländer

$\begin{array}{cccccc}\text { Jahr Ausgaben } & \begin{array}{c}\text { Veränd. } \\ \text { in } \%\end{array} & \begin{array}{ccc}\text { Beitragspflichtige } \\ \text { Einnahmen }\end{array} & \begin{array}{c}\text { Veränd. } \\ \text { in } \%\end{array} & \begin{array}{c}\text { Beitrags- } \\ \text { einnahmen }\end{array} & \begin{array}{c}\text { Veränd. } \\ \text { in } \%\end{array}\end{array}$

\begin{tabular}{|rrrrrr|}
\hline 1.975 & - & 19.848 & - & 2.158 & - \\
\hline 2.900 & 46,8 & 25.441 & 28,2 & 2.806 & 30,0 \\
\hline 3.222 & 11,1 & 29.642 & 16,5 & 3.253 & 15,9 \\
\hline 3.776 & 18,3 & 32.090 & 8,9 & 3.581 & 11,5 \\
\hline 4.453 & 17,9 & 32.178 & 0,3 & 3.687 & 3,0 \\
\hline 4.696 & 6,2 & 32.898 & 2,1 & 3.891 & 6,5 \\
\hline
\end{tabular}

Quelle: Eigene Darstellung nach: BMG, KJ1; für 1996: KV45 1. - 4. Quartal.

\section{Ziel der Ersatzkassen}

Erklärtes Ziel der Ersatzkassen ist es, ihren Versicherten auch zukünftig einen umfassenden Krankenversicherungsschutz auf qualitativ hohem Niveau zu möglichst günstigen Beitragssätzen zu bieten. Um dies vor dem Hintergrund der immer schmaler werdenden Finanzierungsbasis der GKV zu erreichen, sind neben den geschilderten Maßnahmen zur Konsolidierung der GKV-Ost langfristig angelegte Strategien zur Weiterentwicklung des Finan- 
zierungssystems, zur Gestaltung der Leistungserbringung und zur Stärkung wettbewerblicher Strukturen in der GKV notwendig.

Dabei geht es nicht darum, per se mehr Geld in das System zu bringen, denn es kann nicht die Lösung sein, die bestehenden unwirtschaftlichen Strukturen im Gesundheitswesen mit zusätzlichen finanziellen Mitteln am Leben zu erhalten. Strategie der Ersatzkassen ist es vielmehr, durch die Einführung geeigneter Anreizsysteme die umfangreichen Wirtschaftlichkeitsreserven im Gesundheitswesen zu mobilisieren.

Experten schätzen, daß sich die Wirtschaftlichkeitsreserven im Gesundheitswesen in zweistelliger Milliardenhöhe bewegen. So führen die in den einzelnen Versorgungsbereichen bestehenden Überkapazitäten häufig zur Überversorgung. Medizinisch nicht indizierte Eingriffe sowie unnötige Mehrfachdiagnostik und -therapie sind nicht nur unwirtschaftlich: Jede medizinisch nicht notwendige Maßnahme belastet auch die Patienten. Wirtschaftlichkeitsreserven stecken darüber hinaus auch in den unwirtschaftlichen Strukturen, wie z. B. der fehlenden Verzahnung der Leistungsbereiche und der mangelhaften Kooperation der Leistungserbringer.

In ihrem Konzept einer solidarischen Wettbewerbsordnung haben die Ersatzkassen gezeigt, wie durch eine verstärkte wettbewerbliche Ausrichtung Effizienz und Qualität der Versorgung erhöht werden können. Die Rahmenbedingungen für eine verstärkte wettbewerbliche Orientierung sind zwar nach wie vor unzureichend. Mit den GKV-Neuordnungsgesetzen hat der Gesetzgeber weiterhin verhindert, daß sich in den Vertragsverhandlungen gleichgewichtige Partner gegenüberstehen. Statt dessen wurde durch verschiedene Zugeständnisse, wie z. B. feste Punktwerte und Abschaffung der Arznei- und Heilmittelbudgets, die Seite der Leistungserbringer noch zusätzlich gestärkt.

Eine erste praktische Umsetzung ihrer Idee einer verstärkten Wettbewerbsorientierung gelang den Ersatzkassen in den Modellversuchen "Medizinische Qualitätsgemeinschaften" in Rendsburg und im Ried. Ziel dieser Projekte mit niedergelassenen Ärzten ist es, die Transparenz und die Arbeitsteilung im Gesundheitswesen zu verbessern. Bei Erhalt der freien Arztwahl werden durch ein abgestimmtes Verhalten aller Fachgruppen die Versicherten kompetent, schnell, umfassend und auf der richtigen Ver- 
sorgungsebene betreut. Die Wirtschaftlichkeit der Versorgung wird erhöht, indem z. B. Krankenhauseinweisungen vermieden werden können. Die Ärzte werden honoriert, wenn sie durch die Qualifizierung der Arzneimittelverordnungen oder durch die Vermeidung von unnötigen Untersuchungen, Behandlungen und Krankenhauseinweisungen medizinische und ökonomische Erfolge erzielen. Durch diesen Anreiz wird die Flucht in die medizinisch unsinnige und für alle Seiten unbefriedigende Leistungsmenge beendet.

\section{Keine weitere Privatisierung}

Elementare Voraussetzung für den Erhalt der sozialen Krankenversicherung ist, daß es nicht zu einer Entsolidarisierung der Versichertengemeinschaft kommt. Das Solidarprinzip garantiert allen Versicherten einen einheitlichen Leistungsanspruch und schützt sie vor finanzieller Überforderung im Krankheitsfall. Die Leistungsgewährung erfolgt unabhängig von der Höhe der gezahlten Beiträge allein nach der medizinischen Notwendigkeit. Die Höhe der Beiträge richtet sich ausschließlich nach der finanziellen Leistungsfähigkeit der Versicherten. Alter, Geschlecht und gesundheitliche Risiken bleiben im Gegensatz zu den risikoäquivalenten Prämien der PKV bei der Beitragsbemessung unberücksichtigt. So treten in der sozialen Krankenversicherung die finanziell Stärkeren für die finanziell Schwächeren, die Alleinstehenden für die Familien und die Jüngeren für die Älteren ein.

Mit Einführung der typischen PKV-Elemente, wie z. B. Beitragsrückerstattung oder Selbstbehalte und der deutlichen Ausweitung von Zuzahlungen, wurden in den Spargesetzen die Weichen jedoch in Richtung Entsolidarisierung gestellt. Die Hoffnung, über diese systemfremden Instrumente mehr Wirtschaftlichkeit oder Transparenz in die GKV zu bringen, ist trügerisch. Zwar werden durch den Ausbau von Zuzahlungen neue Finanzierungsquellen erschlossen, doch dies einseitig zu Lasten der erkrankten Versicherten. Sinnvolle Steuerungswirkungen können damit nicht erzielt werden. In einem derartigen Vorgehen steckt sozialer Sprengstoff, denn die Akzeptanz des Systems wird zunehmend gefährdet: Freiwillig Versicherte zahlen bereits heute sehr hohe Krankenversicherungsbeiträge und müssen nun bei Leistungsbedarf nicht unerhebliche Teile ihrer Behandlungskosten noch zusätzlich selbst aufbringen. Es fragt sich, ob ihre Bereitschaft zur 
Solidarität, die für das Gesamtsystem von elementarer Bedeutung ist, damit nicht überstrapaziert wird.

Zudem ist zu bedenken, daß derartige Instrumente der Privatversicherung in einem solidarisch finanzierten System nicht funktionieren. In der GKV gibt es weder alters- oder morbiditätsabhängige Tarife noch Leistungsausschlüsse. Eine Risikoentscheidung in der sozialen Krankenversicherung ist weder gewollt noch aufgrund des Kontrahierungszwangs und des Bedarfsprinzips möglich. Die PKV-Elemente in der GKV kollidieren allerdings nicht nur mit dem Prinzip der solidarischen Finanzierung. Sie führen auch zu einer zusätzlichen Belastung derjenigen Versicherten, die aus finanziellen oder gesundheitlichen Gründen diese Tarife nicht wählen können.

\section{Ja zur paritätischen Finanzierung}

Die Politik nutzt den Weg der Privatisierung von Gesundheitsleistungen, um Finanzquellen zu erschließen, die nicht die Arbeitgeberbeiträge zur Krankenversicherung tangieren. An den Gesundheitsleistungen, die der Versicherte aus eigener Tasche zu finanzieren hat, ist der Arbeitgeber nicht mehr beteiligt. Bevor auf diesem Weg das Prinzip der paritätischen Finanzierung in der GKV schleichend verlassen wird, sollte eine offene Diskussion über die Bedeutung der Parität in einem solidarisch finanzierten System geführt werden.

Eine völlige Abschaffung des Arbeitgeberbeitrags im Sinne einer einmaligen Umwandlung in Einkommensbestandteile wäre aus ökonomisch-theoretischer Sicht konsequent: Diese Forderung schließt an die alte ökonomische Erkenntnis an, daß es im strengen theoretischen Sinne einen Arbeitgeberbeitrag nicht gibt, sondern diese Belastung als Kalkulationsgrundlage den Lohnkosten zuzuordnen ist. Dies wird auch durch die Systematik der volkswirtschaftlichen Gesamtrechnung belegt, in der die Arbeitgeberbeiträge der Lohnquote zugeordnet werden. Diese ökonomisch richtige Argumentation kann jedoch den sozialpolitischen Sprengsatz dieses Vorschlags nicht entkräften. Dieser Vorschlag würde nämlich ein Zurückziehen der Arbeitgeber aus der Gesamtverantwortung für die Stabilität der sozialen Krankenversicherung bedeuten und die finanziellen Zukunftsrisiken allein auf die Versicherten übertragen. Die Ausgabenentwicklung im $\mathrm{Ge}-$ sundheitswesen wäre damit auch für die Wirtschaftspolitik nicht 
mehr von Interesse. Zudem wäre die Aufteilung der Beitragslast zukünftig extrem abhängig von der ökonomischen Situation und der Machtverteilung zwischen den Tarifpartnern. Die Tarifverhandlungen würden zusätzlich erheblich belastet.

Ein rundum systemadäquater Vorschlag, der die paritätische $\mathrm{Fi}$ nanzierung sichert und unter den Bedingungen einer verstärkt wettbewerblich ausgerichteten Krankenversicherung ordnungspolitisch konsequent wäre, ist die Orientierung des Arbeitgeberanteils am jeweils hälftigen durchschnittlichen GKV-Beitrag. Dieser Vorschlag setzt am Problem des kassenindividuellen Arbeitgeberbeitrags an, der in einer wettbewerblich orientierten GKV die Be- bzw. Entlastung der Unternehmer von der Wahlentscheidung des Mitarbeiters abhängig macht. Daraus resultiert ein betriebswirtschaftliches Interesse der Arbeitgeber an der Beeinflussung der Kassenwahl. Ziel muß es deshalb sein, den Arbeitgeberbeitrag zu neutralisieren, um die Wahlfreiheit der Versicherten sicherzustellen. Die Orientierung des Arbeitgeberbeitrags am jeweils hälftigen durchschnittlichen GKV-Beitragssatz garantiert diese Neutralität. Sie ist wettbewerbsneutral zwischen den Krankenkassen, zwischen den Unternehmen sowie zwischen den Regionen. Dieses Konzept stabilisiert die Lohnnebenkosten auf einer verläßlichen und kalkulierbaren Grundlage und schafft durch die Notwendigkeit einer Stichtagsentscheidung (z. B. 1. Januar) für eine definierte Periode kalkulatorische Stabilität.

Die Stabilität des Gesamtsystems spielt bei den Überlegungen der Ersatzkassen jedoch nicht nur bei der Frage der Gestaltung des Arbeitgeberbeitrags eine wichtige Rolle. Sie ist bei jeder Veränderung des Finanzierungssystems (versicherter Personenkreis, Beitragsbemessungs- und Versicherungspflichtgrenze sowie Beitragsbemessungsgrundlage) $\mathrm{zu}$ beachten. Insgesamt betrachtet, ist das Finanzierungssystem in der GKV so weiterzuentwickeln, daß es auch den zukünftigen Herausforderungen eines expandierenden Gesundheitswesens bei begrenzten finanziellen Ressourcen gewachsen ist. Zentrale Kriterien sind dabei aus Sicht der Ersatzkassen, neben dem Aspekt der Mittelschöpfung, vor allem Gerechtigkeit sowie Effizienz der getroffenen Maßnahme. So ist z. B. eine Beitragsgestaltung abzulehnen, wenn sie im Vergleich zu den erzielten Einnahmen zu hohe Erhebungskosten verursacht oder dem Gerechtigkeitspostulat widerspricht. 


\section{GKV auch für Selbständige und Beamte?}

Zur Stärkung der Solidargemeinschaft der GKV könnte der versicherte Personenkreis auf weitere Bevölkerungsgruppen, $\mathbf{z}$. B. auf Selbständige und/oder Beamte ausgeweitet werden. Unter dem Aspekt der Erschließung neuer Finanzierungsquellen ist zu prüfen, ob es sich bei den potentiellen neuen Mitgliedern um Nettozahler oder um Nettoempfänger handelt; daneben ist immer die sozial- und gesellschaftspolitische Akzeptanz zu prüfen.

Problematisch bei Einbeziehung der Gruppe der Selbständigen ist der Tatbestand, daß es eine dem Bruttoeinkommen bei den Nichtselbständigen vergleichbare Größe bei den Selbständigen nicht gibt. Derzeit erfolgt ggf. die Regeleinstufung für Selbständige auf der Höhe der Beitragsbemessungsgrenze. Die Untergrenze liegt bei $75 \%$ der Bezugsgröße, d. h. 1997 bei $3.203 \mathrm{DM}$ im Westen bzw. 2.730 DM im Osten. Da Selbständige weit größere Möglichkeiten haben, ihr steuerpflichtiges Einkommen zu reduzieren, würde es damit zu einer nicht vertretbaren Ungleichbehandlung im Vergleich zu den Nichtselbständigen kommen. Die Einbeziehung von Selbständigen ist daher weder unter Gerechtigkeitsaspekten noch unter fiskalischen Gesichtspunkten anzustreben.

Seit Inkrafttreten des Gesundheitsreformgesetzes haben Beamte kein gesondertes Beitrittsrecht zur GKV. Sie können sich lediglich im Anschluß an eine vorangegangene Mitgliedschaft oder Familienversicherung gesetzlich weiterversichern. Da sich das Klientel der GKV in erster Linie aus dem Kreis der unselbständig Beschäftigten und deren Angehörigen zusammensetzt, wäre aus systematischen Erwägungen ihre Einbeziehung in die GKV durchaus sachgerecht. Statt Beihilfe müßten die Beamten von ihrem Dienstherrn einen dem Arbeitgeberanteil äquivalenten $\mathrm{Zu}$ schuß erhalten. Auch aus Gründen der Gerechtigkeit wäre die Einbeziehung der Beamten in die GKV zu befürworten. Schließlich ist nicht einzusehen, daß gerade Beamte als unmittelbare Staatsdiener sich der im Sozialstaat verankerten Solidarität entziehen und sich in der PKV absichern können.

Die Einbeziehung der Beamten in die Versicherungspflicht birgt eine Reihe verfassungsrechtlicher Probleme (Artikel 33 [5] GG Grundsätze des Berufsbeamtentums, Artikel 20 [1] GG Vertrauensschutz der [bisher] privat Versicherten, Artikel 12 [1], 14 GG 
Berufs- und Gewerbefreiheit der PKV-Unternehmen). Selbst wenn diese Probleme durch entsprechende Übergangsregelungen gelöst werden könnten, ist jedoch zumindest in naher Zukunft nicht damit zu rechnen, die notwendigen politischen Mehrheiten für eine derartige Neugestaltung der Versorgung der Beamten zu finden. Auch unter rein fiskalischen Gesichtspunkten ist aus Sicht der GKV die Einbeziehung der Beamten eher zurückhaltend zu bewerten. Die Gruppe der Beamten verursacht nämlich aufgrund der überdurchschnittlichen, krankheitsbedingten Arbeitsunfähigkeit auch vermutlich überdurchschnittlich hohe Kosten. Da zudem die Bruttobezüge der Beamten im Vergleich zu denen der Angestellten geringer ausfallen, ist zu vermuten, daß durch deren Einbeziehung voraussichtlich eher Nettoempfänger in die GKV kommen.

Die aktuell diskutierte Aufhebung der Versicherungsfreiheit für geringfügig Beschäftigte würde ebenfalls den Personenkreis der GKV erweitern und für die GKV neue Finanzierungspotentiale erschließen. Derzeit besteht bis zu einem Verdienst von $610 \mathrm{DM}$ im Monat (bzw. $520 \mathrm{DM}$ im Osten) für die Beschäftigten Versicherungsfreiheit ( $\$ 7$ SGB V). Die Einbeziehung dieser Arbeitsverhältnisse wird zur Zeit vor allem deshalb diskutiert, weil sie zunehmend ihren Charakter als individuelle Zuverdienstmöglichkeiten verlieren und zu einer strategischen Möglichkeit für Arbeitgeber zur Umgehung der Sozialversicherungspflicht werden.

Die Zahl der geringfügig Beschäftigten wird mittlerweile auf 5 Mio. geschätzt. Bei einem angenommenen durchschnittlichen Beitragssatz von $13,7 \%$ und unter der Annahme, daß alle Versicherten den West-Höchstsatz von $610 \mathrm{DM}$ verdienen, entspricht dies einem Einnahmenvolumen von 4,2 Mrd. DM. Die tatsächlich zusätzlich zu realisierenden Einnahmen der GKV werden jedoch deutlich geringer ausfallen, da zum einen die Bemessungsgrenze in den neuen Bundesländern niedriger liegt und zum anderen ein großer Teil der geringfügig Beschäftigten schon heute GKVBeiträge zahlt (im Rahmen ihrer Haupttätigkeit oder als Arbeitslose bzw. Rentner). Der weitaus größte Teil ist jedoch zur Zeit bereits beitragsfrei über eine Familienversicherung abgesichert. Auch vor diesem Hintergrund ist unter fiskalischen Gesichtspunkten eine Neuregelung der Versicherungsfreiheit geringfügig Beschäftigter zu begrüßen. 

GKV

Durch Veränderung von Versicherungspflicht- und/oder Beitragsbemessungsgrenze läßt sich ebenfalls der Umfang der solidarischen Finanzierung in der GKV verändern. Die Beitragsbemessungsgrenze, d. h. die Grenze, bis zu der die Beiträge als prozentualer Anteil des Einkommens entrichtet werden, ist zugleich Versicherungspflichtgrenze, ab der die Versicherten wählen können, ob sie als freiwillig Versicherte weiter in der GKV bleiben, sich in der PKV oder gar nicht versichern wollen. Die Festlegung dieser Grenzen ist wissenschaftlich nicht ableitbar, sondern das Ergebnis politischer Konventionen.

Nach der heutigen Rechtslage ist die Höhe der Beitragsbemessungsgrenze in der GKV an die in der gesetzlichen Rentenversicherung gekoppelt. In der Rentenversicherung wird die Beitragsbemessungsgrenze jährlich auf das Doppelte der allgemeinen Bemessungsgrundlage, die dem durchschnittlichen Bruttoarbeitsverdienst aller Versicherten entspricht, festgesetzt. Derzeit liegt sie bei 8.200 DM im Westen und 7.100 DM im Osten (Übersicht 4). In der GKV beträgt die Beitragsbemessungsgrenze $75 \%$ dieser Größe, liegt also zur Zeit (1997) bei 6.150 DM in den alten Bundesländern und 5.325 DM in den neuen Bundesländern. 


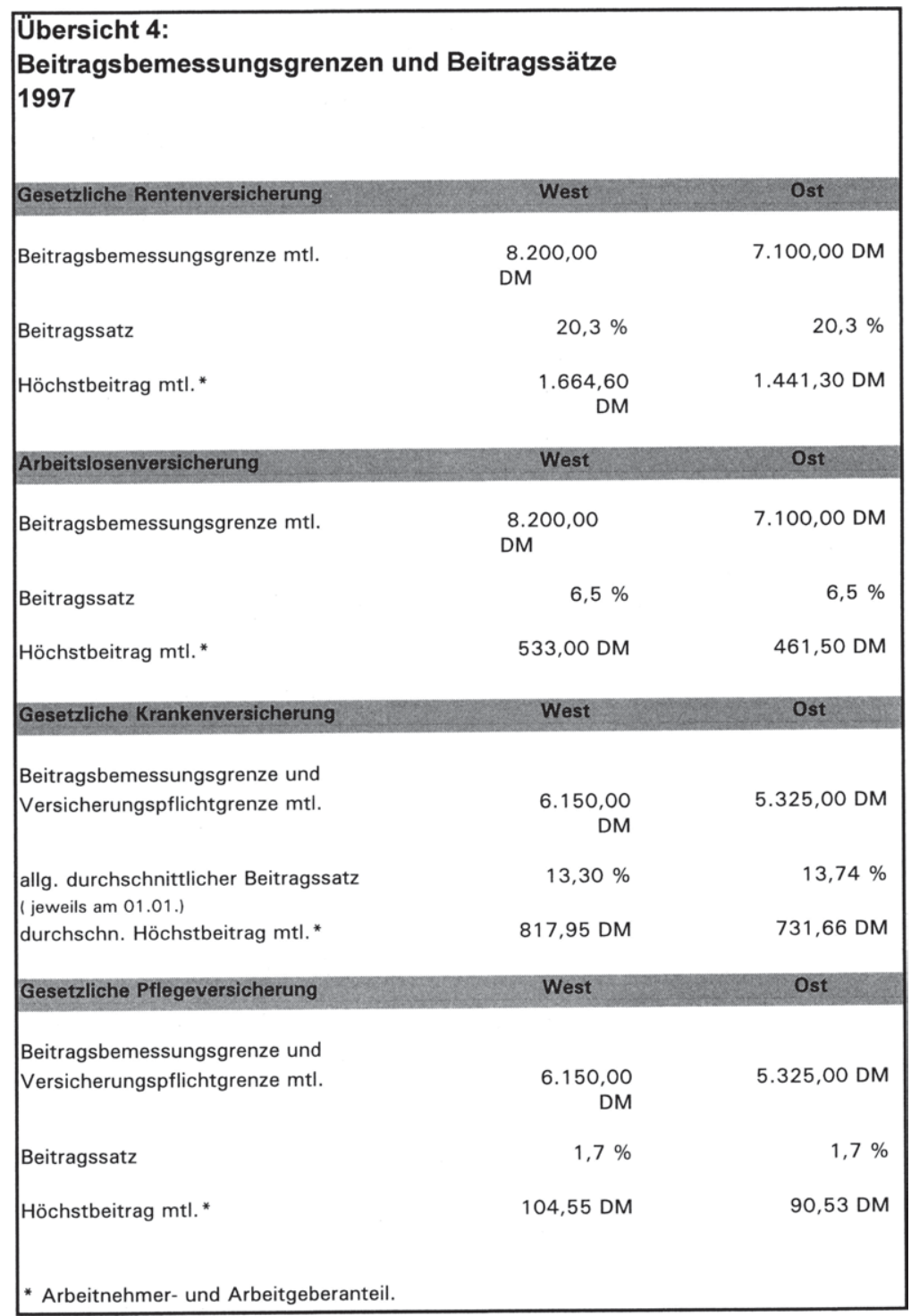

\section{Quelle: Eigene Darstellung}


Bei einer Erhöhung der Beitragsbemessungsgrenze ist $\mathrm{zu}$ berücksichtigen, daß dem dadurch betroffenen Personenkreis trotz der höheren Beitragszahlung keine äquivalente Erweiterung oder Verbesserung des Versicherungsschutzes gegenübersteht. Die Finanzierung der GKV erfolgt schließlich in erster Linie nach dem Grundsatz der finanziellen Leistungsfähigkeit und nicht wie in der PKV nach Kriterien der individuellen Risikoäquivalenz. Zwar enthält die GKV auch Elemente einer gewissen Äquivalenz von Beiträgen und Leistungen. So bestand zur Zeit ihrer Gründung die wichtigste Leistung in der Gewährung von Krankengeld, dessen Höhe sich nach der Höhe der Beiträge richtet. Auch heute noch beruhen die Einkommensersatzleistungen der Krankenkassen auf dem Äquivalenzprinzip.

Auch die Existenz einer Beitragsbemessungsgrenze in der GKV entspricht diesem Prinzip, da der einkommensbezogene Solidarausgleich nur bis zu dieser Grenze erfolgt. Oberhalb der Beitragsbemessungsgrenze werden keine weiteren Beiträge mehr erhoben, d. h. die Leistungsfähigkeit des Versicherten (entspricht der Höhe seines Einkommens) bleibt unberücksichtigt. Ab dieser Grenze geht man davon aus, daß die Höhe des Beitrags in keinem annehmbaren Verhältnis mehr zum zu erwartenden Gegenwert, d. h. der Leistung im Krankheitsfall steht. Eine Erhöhung der Beitragsbemessungsgrenze sollte deshalb nur erfolgen, wenn plausible Gründe dafür vorliegen.

An der immer wieder erhobenen Forderung, die Beitragsbemessungsgrenze auf die in der Rentenversicherung anzuheben, lassen sich die konkreten problematischen Folgen einer derartigen Änderung verdeutlichen: Versicherte in den alten Bundesländern mit einem Einkommen oberhalb der Beitragsbemessungsgrenze (6.150 DM) zahlen zur Zeit bei einem Beitragssatz von 13,7\%, einschließlich des Arbeitgeberanteils, monatlich 843 DM an Krankenversicherungsbeitrag. Bei einer Erhöhung der Grenze auf 8.200 DM (Niveau in der gesetzlichen Rentenversicherung) müßten diese Versicherten, falls sie aufgrund ihrer Einkommenshöhe nicht zu Pflichtversicherten würden, bei konstantem Beitragssatz 1.123 DM einschließlich des Arbeitgeberanteils, d. $h$. monatlich fast $300 \mathrm{DM}$ mehr an Krankenversicherungsbeitrag entrichten. Diesen höheren Zahlungen stünden jedoch keinerlei Gegenleistungen gegenüber. 
Bei einer Erhöhung der Beitragsbemessungsgrenze, wie bei allen anderen Veränderungen des Finanzierungssystems, sind die Auswirkungen der zwingend notwendigen Übergangsregelungen (Bestandsschutz) zu berücksichtigen. So wird in diesem Fall die Zahl der Abwanderungen in die PKV entsprechend hoch sein, wobei zu vermuten ist, daß tendenziell jüngere und gesunde Versicherte die GKV verlassen werden. Auf der anderen Seite ist zu erwarten, daß insbesondere ältere und kranke PKVVersicherte die Chance nutzen werden, um in die GKV aufgenommen zu werden. Damit kommt es zu einer Entmischung der Risiken zu Lasten der GKV, deren finanzielle Folgen zumindest mittelfristig deutlich spürbar sein werden.

Plausible Gründe für eine Anhebung der Beitragsbemessungsgrenze in der GKV gibt es zur Zeit nicht. Zwar würde eine derartige Anhebung zu Mehreinnahmen führen, die aber unter den günstigsten Annahmen (ohne Übergangsregelungen und bei tendenzieller Überschätzung der Einkommen) deutlich weniger als einen Beitragssatzpunkt ausmachen werden. Unter der Annahme konstanter Ausgaben könnten damit die Beitragssätze zwar möglicherweise reduziert und so die unteren Einkommensschichten entlastet werden, eine Anhebung der Beitragsbemessungsgrenze würde jedoch prinzipiell nichts an der ungleichmäßigen und daher vermeintlich ungerechten Belastung der GKV-Versicherten ändern, deren Einkünfte nahe der Beitragsbemessungsgrenze liegen. Der betroffene Personenkreis hätte sich lediglich verschoben. Um die Akzeptanz des Gesamtsystems, das auf die Solidarität gerade der höher Verdienenden angewiesen ist, nicht zu gefährden, sollte aus den genannten Gründen bei der Weiterentwicklung des Finanzierungssystems die Beitragsbemessungsgrenze nicht verändert werden.

\section{Aufhebung der Versicherungspflichtgrenze ... bei Beibehaltung der Beitragsbemessungsgrenze}

Aus Gründen der Gerechtigkeit stellt sich die Frage, warum es einer Gruppe, nämlich den freiwillig Versicherten, gestattet wird, sich je nach Belieben der umfassenden Solidarität in der GKV zu entziehen. Dies ist um so problematischer, als tendenziell insbesondere die guten Risiken, also junge, alleinstehende, gesunde Versicherte, zur PKV wechseln. Eine Aufhebung der Versicherungspflichtgrenze in der GKV würde dies verhindern. Unab- 
hängig von ihrem Einkommen wären alle Personen, außer Selbständigen und Beamten, in der GKV versicherungspflichtig.

Eine umfassende Versicherungspflicht für alle abhängig Beschäftigten (außer den Beamten) würde zudem zu einer Stärkung der Finanzkraft der GKV führen, da das Finanzierungsvolumen sich unter diesen Bedingungen auf einen größeren Personenkreis verteilen würde. Da hierdurch der durchschnittliche Beitragssatz sinken könnte, würde der Arbeitgeberanteil insgesamt betrachtet in etwa konstant gehalten werden können. Voraussetzung dafür ist, daß die Beitragsbemessungsgrenze nicht gleichzeitig angehoben wird, denn nur bei Beibehaltung der derzeitigen Beitragsbemessungsgrenze werden sich die während der Übergangsregelung zu erwartenden Abwanderungen in Grenzen halten.

\section{Keine Erweiterung der Beitragsbemessungsgrundlage}

Insbesondere vor dem Hintergrund der aktuell wegbrechenden Einnahmen in der GKV muß schließlich die Frage gestellt werden, ob die bisherige ausschließliche Beitragsschöpfung aus Arbeitseinkommen und Lohnersatzleistungen auch für die Zukunft tragbar ist. So könnte durch eine entsprechende Erweiterung der Beitragsbemessungsgrundlage erreicht werden, daß die Finanzierungsgrundlage der GKV weniger konjunkturabhängig ist.

Unter dem Aspekt der möglichst vollständigen Ausschöpfung zusätzlicher Finanzierungspotentiale wird deshalb vorgeschlagen, die Beitragsbemessung in der GKV auf weitere Einkunftsarten auszuweiten. So könnten z. B. neben dem Einkommen aus unselbständiger Tätigkeit im Prinzip alle weiteren steuerpflichtigen Einkünfte zur Beitragspflicht herangezogen werden (7 Einkunftsarten nach § 2 EStG). Dazu zählen neben dem Arbeitsentgelt Einkünfte aus Land- und Forstwirtschaft, aus Gewerbebetrieb, aus selbständiger Arbeit, aus Kapitalvermögen, aus Vermietung und Verpachtung sowie sonstige Einkünfte.

Aus Gerechtigkeitsgründen wäre eine derartige Erweiterung der Bemessungsgrundlage aus zwei Gründen möglicherweise sinnvoll:

Die horizontale Ungleichbehandlung im heutigen System würde gemildert. Wird nur das Arbeitsentgelt der Beitragsbemessung zugrunde gelegt, kann es dazu kommen, daß Versicherte mit 
gleichem Gesamteinkommen unterschiedlich hohe Krankenversicherungsbeiträge zu leisten haben, weil die einen in erster Linie (beitragspflichtige) Erwerbseinkommen und die anderen vor allem Einkünfte aus anderen (nicht beitragspflichtigen) Einkommensarten beziehen.

Die Ungleichbehandlung von freiwillig und Pflichtversicherten bei der Beitragsbemessung würde beseitigt: Bei freiwillig Versicherten wird de jure bereits heute bei der Beitragsbemessung nicht nur das Arbeitsentgelt zugrunde gelegt, sondern ihre "gesamte wirtschaftliche Leistungsfähigkeit" (§ 240 [1] SGB V). Die Erwerbseinkommen dieses Personenkreises liegen zwar in der Regel über der Beitragsbemessungsgrenze, so daß de facto nur in Ausnahmefällen weitere Einkunftsarten einbezogen werden. Anders wirkt dies bei freiwillig versicherten Rentnern, für die ebenfalls bereits heute bei der Beitragsbemessung alle sonstigen Einnahmen berücksichtigt werden ( $\$ 238$ a SGB V). Sie zahlen Krankenversicherungsbeiträge aus allen erzielten Einkünften, also auch aus Einnahmen, die aus privater Eigenvorsorge resultieren, wie $z$. B. Zins- und Mieteinnahmen.

Die Nachteile einer Erweiterung der Beitragsbemessungsgrundlage überwiegen jedoch: So ist unter Finanzierungsaspekten zu berücksichtigen, daß die Vermögensverteilung in der Bundesrepublik sehr ungleich ist. Tendenziell werden Versicherte mit hohem Vermögen bereits mit ihren Arbeitseinkommen über der Beitragsbemessungsgrenze liegen. Zumindest bei unveränderter Beitragsbemessungsgrenze wird damit das Volumen des zusätzlichen Finanzierungspotentials eher gering ausfallen. Da zudem ein großer Teil des in den unteren und mittleren Einkommensschichten vorhandenen Vermögens aus selbstgenutzten Eigenheimen besteht, aus denen keine Vermögenseinkünfte fließen, ist auch hieraus kein zusätzliches Potential zur Beitragsschöpfung zu erwarten.

Die Erfassung sämtlicher Einkunftsarten bei der Beitragsbemessung ist aber auch aus systemimmanenten Gründen höchst problematisch. Die Krankenkassen werden nicht in der Lage sein, eigenständig bei den Versicherten die Höhe sämtlicher beitragspflichtiger Einkünfte zu ermitteln. Nach geltendem Recht haben ca. 1,5 Mio. Arbeitgeber in der Bundesrepublik die Gesamtsozialversicherungsbeiträge ihrer Beschäftigten zu ermitteln und an die zuständigen Einzugsstellen abzuführen. Die Krankenkassen er- 
halten die entsprechenden Beiträge als Gesamtsumme, d. h., ein Einzelnachweis je Mitglied erfolgt nicht.

Dieses verwaltungsfreundliche Verfahren ist bei einer Erweiterung der Beitragsbemessungsgrundlage nicht mehr möglich: Zukünftig müßten ca. 27 Mio. Arbeitnehmer ihre Beiträge selbst zahlen. Um die eingehenden Buchungen zu kontrollieren, müßten die Krankenkassen sich jeden Monat nicht nur die Lohn- und Gehaltsabrechnungen vorlegen lassen, sondern auch Nachweise über die übrigen Einkünfte der Versicherten. Die Erfassungsintensität wird dabei nicht unabhängig von der Art der Einkünfte sein. Problematisch wird dies insbesondere für Einnahmen, die nicht regelmäßig fließen, wie z. B. Zinseinkünfte, die häufig erst am Ende des Jahres fällig werden. Der Verwaltungsaufwand der Kassen wäre äußerst hoch: Neben der inhaltlichen Kontrolle der eingegangenen Zahlungen müßten nicht nur Mahnbescheide verschickt und Säumniszuschläge erhoben werden, sondern z. B. auch Maßnahmen eingeleitet werden, wenn Versicherte sich weigern, Beiträge zu entrichten. Ein Ausschluß aus der Versicherung ist bei privat Versicherten bzw. freiwillig Versicherten möglich, bei Pflichtversicherten wohl kaum.

Auch die versicherungsrechtliche Einstufung der Mitglieder („pflichtversichert" oder „freiwillig versichert"), die zur Zeit von den Arbeitgebern vorgenommen wird, wäre nun Aufgabe der Krankenkassen. Mit den starken, oft kurzfristigen Schwankungen einer erweiterten Beitragsbemessungsgrundlage wären für bestimmte Versichertengruppen weit häufigere Statuswechsel mit entsprechenden Folgen (z. B. Wechselmöglichkeiten zur PKV) verbunden.

Im Sinne von Erhebungsbilligkeit und Beitragsgerechtigkeit wird vermutlich nur eine nachträgliche Beitragsbemessung anhand von Einkommensteuerbescheiden in Frage kommen, was gegenüber heute, wo die Beitragserhebung direkt bei der Lohn- bzw. Gehaltszahlung erfolgt, eine zeitliche Verzögerung von mindestens einem Jahr bedeuten würde. Der faktisch vorhandene "gläserne Geldbeutel" durch den Quellenabzug bei den Einkünften aus nichtselbständiger Arbeit sowie die zu erwartende Kapitalflucht lassen zudem erhebliche unerwünschte Verteilungswirkungen, insbesondere zu Lasten der Bezieher von Einkünften aus nichtselbständiger Tätigkeit, befürchten. Außerdem würde diese Lösung eine (weitere) Abkehr von der paritätischen Finanzierung 
bedeuten, da der Arbeitgeberbeitrag sich lediglich auf die Erwerbseinkommen bezieht.

Die Erweiterung der Beitragsbemessungsgrundlage durch die Einbeziehung sämtlicher Einkunftsarten ist jedoch auch unter dem Gesichtspunkt der Belastungsgerechtigkeit fragwürdig. Durch eine derartige Neuregelung werden lediglich Versicherte betroffen, deren Arbeitseinkommen derzeit unterhalb der Beitragsbemessungsgrenze liegen. Ihre zusätzlichen Einkünfte würden beitragspflichtig. Versicherte mit Einkommen oberhalb der Beitragsbemessungsgrenze würden keine zusätzlichen Belastungen erfahren; im Gegenteil, sie würden durch eine mögliche Beitragssenkung aufgrund der erzielten Mehreinnahmen sogar noch entlastet. Das heißt, die Einbeziehung weiterer Einkunftsarten bei gleichzeitiger Beibehaltung der Beitragsbemessungsgrenze würde zu einer sozialpolitisch nicht vertretbaren Umverteilung von den geringer Verdienenden zu den höher Verdienenden führen.

Aus den soeben genannten Gründen ist es sinnvoll, die Beitragsbemessungsgrundlage in der GKV, wie sie derzeit der Rechtslage für Pflichtversicherte entspricht und auch bei Aufhebung der Versicherungspflichtgrenze für alle abhängig Beschäftigten gelten könnte, nicht zu erweitern. Die primär lohnbezogene Beitragsbemessungsgrundlage schließt eng an die Definition der GKV als Arbeitnehmerversicherung an. Sie stellt den Schutz abhängig Erwerbstätiger in den Mittelpunkt sozialstaatlich organisierter Sicherung. Das Arbeitsentgelt als Bemessungsgrundlage bietet zudem den Vorteil einer verläßlichen Kalkulationsbasis, da zum einen die Arbeitnehmerentgelte kaum manipulierbar und zum anderen Arbeitnehmer- und Arbeitgeber-Beitragsanteile klar zuzuordnen sind. Darüber hinaus ist der Erhebungsaufwand im Vergleich zu anderen Bemessungsgrundlagen gering.

\section{Fazit}

Die dargestellten Ungerechtigkeiten bei der Beitragsbemessung sowie die Probleme einer primär lohnbezogenen Beitragsbemessungsgrundlage vor dem Hintergrund der steigenden Arbeitslosigkeit machen deutlich, daß das derzeitige Finanzierungssystem der GKV nur eine "second best"-Lösung darstellt. Veränderungen sind an vielen Stellen denkbar, sollten jedoch mit Behutsamkeit angegangen werden, da alle zur Zeit diskutierten Lösungsvorschläge mit Folgeproblemen bzw. neuen Ungerechtigkeiten ver- 
bunden sind. Eine optimale Lösung ist noch nicht gefunden. Es bleibt auch deshalb die Verantwortung bestehen, das Rationalisierungspotential völlig auszuschöpfen, bevor schwierige und politisch sensible finanztechnische Veränderungen geplant werden. 


\section{Finanzierungsoptionen der gesetzlichen Krankenversicherung}

Anita B. Pfaff

\section{Einführung: Ansatzpunkte der Reform der Finanzierung}

Zur Beurteilung von Finanzierungsoptionen der gesetzlichen Krankenversicherung bietet sich eine isolierte Betrachtung der Finanzierungsseite an, auch wenn dadurch mindestens indirekt immer auch die private Krankenversicherung beeinflußt wird bzw. eine Querverbindung zu ihr besteht. In der Praxis werden Finanzierungsalternativen allerdings meist im Zusammenhang mit Veränderungen auf der Leistungsseite diskutiert. Es bieten sich drei verschiedene Gruppen von Finanzierungsoptionen an, die allerdings nicht ohne Beziehung zueinander sind (vgl. auch SVRKAiG 1997):

- Welche Veränderungen im Versichertenkreis sind ins Auge zu fassen?

- Wie ist die Bemessungsgrenze und die Bemessungsgrundlage zu wählen?

- Wie werden die Beiträge kalkuliert?

Das Referat befaßt sich mit einer Diskussion verschiedener Alternativen der Finanzierung der gesetzlichen Krankenversicherung. Dabei wird versucht, auf der Basis von Modellrechnungen für das Jahr 1994 das Ausmaß der möglichen Effekte abzuschätzen.

\section{Versicherten- und Beitragsstruktur der gesetzlichen Krankenversicherung}

Durch die Ausweitung des Versichertenkreises der gesetzlichen Krankenversicherung in der Bundesrepublik Deutschland wird praktisch immer auch der Versichertenkreis der privaten Krankenversicherung mit beeinflußt. $\mathrm{Da}$ in der Bundesrepublik Deutschland fast die gesamte Bevölkerung über einen umfassenden Versicherungsschutz verfügt, kann eine nennenswerte Ausweitung des GKV-Versichertenkreises faktisch nur zu Lasten der privaten Krankenversicherung gehen; nur ein relativ kleiner Kreis wird über „Sondersysteme“ (z. B. Sozialhilfeempfänger, Polizei etc.) abgesichert (vgl. Abbildungen 1a und $1 b$ ). 
Abb. 1a: Bevölkerung nach dem Krankenversicherungsstatus, alte Länder 1995 , in Mio. und in \%

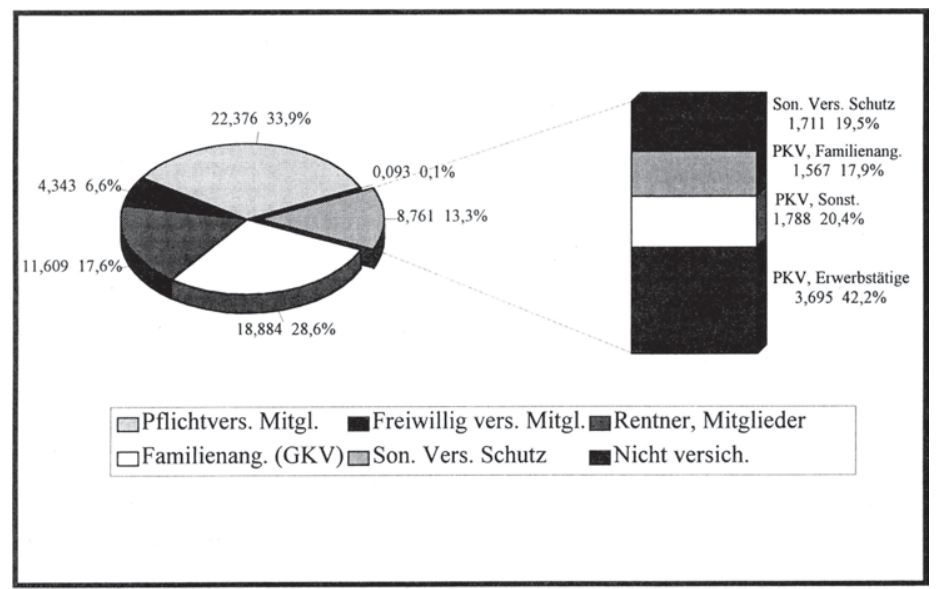

Abb. 1b: Bevölkerung nach dem Krankenversicherungsstatus, neue Länder 1995 , in Mio. und in \%

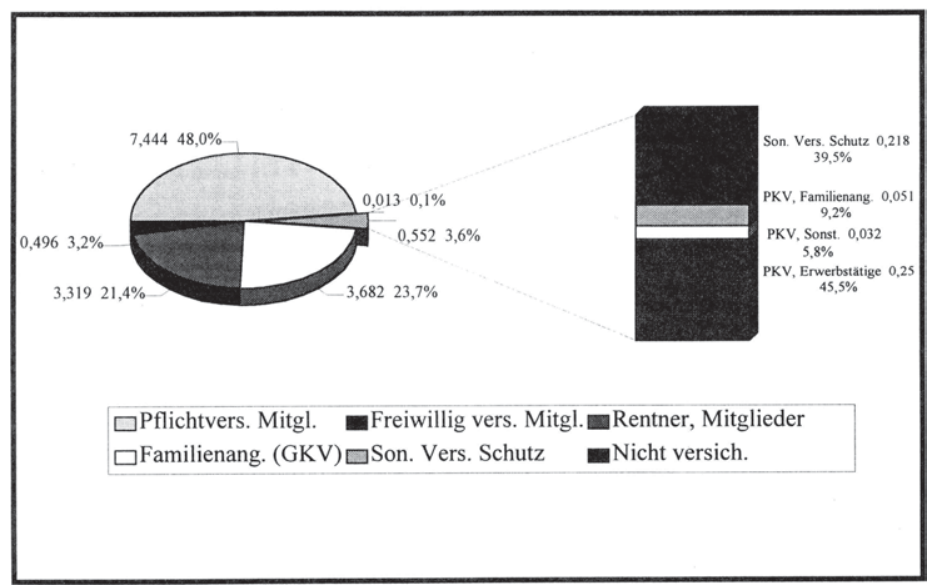

Quelle: Eigene Darstellung nach Statistisches Bundesamt (Hrsg.), Versicherte in der Kranken- und Rentenversicherung 1995, Wiesbaden 1996 
Betrachtet man die Verteilung der Bevölkerung der alten Bundesländer nach dem Versicherungsstatus in Abbildung 1a und die der neuen Länder in Abbildung 1b, so wird auch ersichtlich, welcher Anteil der Bevölkerung heute nicht bereits in der gesetzlichen Krankenversicherung versichert ist. Es folgt, für welchen Personenkreis der GKV-Versicherten eine Wahlmöglichkeit zwischen gesetzlicher und privater Krankenversicherung besteht.

Während mit etwa $90 \%$ der überwiegende Teil der Bevölkerung GKV-versichert ist, besteht doch für die verschiedenen Versichertengruppen ein sehr unterschiedliches Maß, ihren Versicherungsschutz zu wählen.

Sieht man davon ab, daß ein - dauerhafter - Verzicht auf die GKV-Versicherung auch für Pflichtversicherte möglich ist, so steht freiwillig Versicherten der gesetzlichen Krankenversicherung und Teilen der privat krankenversicherten Personen die Möglichkeit offen, zwischen der Versicherungszugehörigkeit in der gesetzlichen und der privaten Krankenversicherung zu wählen. In den alten Ländern waren - nach Erfassung im Mikrozensus - im Jahr 1995 4,3 Mio. Personen oder 6,6 \% der Bevölkerung als Mitglieder freiwillig versichert, in den neuen Ländern 0,5 Mio. oder $3,2 \%$. Dem Versichertenkreis der freiwillig GKV-Versicherten sind allerdings auch die mitversicherten Familienangehörigen dieser Mitglieder zuzurechnen. Bezieht man sich auf die GKV-Daten zur Versichertenstruktur (Bundesministerium für Arbeit und Sozialordnung 1996, S. 204 und 209 ff.), so zeigt sich, daß etwa $25 \%$ der mitversicherten Familienangehörigen in der gesetzlichen Krankenversicherung in den alten und $11 \%$ in den neuen Ländern auf freiwillig versicherte Mitglieder entfallen. Bei einem Mitgliederanteil von $12 \%$ freiwillig versicherter GKV-Mitglieder in den alten und $6 \%$ in den neuen Ländern ist dieser Anteil überproportional hoch. Er entspricht einem Verhältnis von 0,91 mitversicherten Familienangehörigen je freiwillig versichertem Mitglied in den alten und 0,5 in den neuen Ländern, im Vergleich zu nur 0,5 bei den pflichtversicherten Mitgliedern.

Laut Angaben des Mikrozensus sind etwa 7 Mio. Personen in den alten und 0,33 Mio. Personen in den neuen Ländern privat krankenversichert (vgl. Tabelle 1). Mehr als die Hälfte davon, nämlich insgesamt 3,9 Mio., sind Erwerbstätige - 1,8 Mio. Beamte, 0,9 Mio. Angestellte und 1,2 Mio. Selbständige. Unter den mitversicherten Familienangehörigen werden 1,6 Mio. als privat Versi- 
cherte ausgewiesen2; dies entspricht 0,28 mitversicherten Familienangehörigen je „freiwillig" privat Versicherten (= Erwerbspersonen und Nichterwerbspersonen außer Familienangehörigen) (vgl. Statistisches Bundesamt 1996, S. 23 f.). In den alten Ländern liegt die Zahl der mitversicherten Familienangehörigen je Mitglied höher als in den neuen (vgl. Tabelle 1).

Tab. 1: Mitglieder, Versicherte (in 1000) und mitversicherte Familienangehörige je Mitglied nach Versichertenstatus 1995

\begin{tabular}{|c|c|c|c|c|c|}
\hline & $\begin{array}{l}\text { GKV-Pflicht- } \\
\text { mitglieder }\end{array}$ & $\begin{array}{l}\text { GKV Freiw. } \\
\text { Mitglieder }\end{array}$ & $\begin{array}{l}\text { KVdR- } \\
\text { Mitglieder }\end{array}$ & $\begin{array}{l}\text { Privat Versi- } \\
\text { cherte }^{*}\end{array}$ & Insgesamt* \\
\hline \multicolumn{6}{|l|}{ Alte Länder } \\
\hline Mitglieder* & 23.903 & 5.065 & 11.735 & 5.482 & 46.185 \\
\hline $\begin{array}{l}\text { Familien- } \\
\text { angehörige }\end{array}$ & 11.983 & 4.596 & 1.467 & 1.567 & 19.613 \\
\hline Versicherte & 35.886 & 9.661 & 13.202 & 7.049 & 65.798 \\
\hline $\begin{array}{l}\text { Familienan- } \\
\text { gehörige je } \\
\text { Mitglied }\end{array}$ & 0,50 & 0,91 & 0,13 & 0,29 & 0,42 \\
\hline \multicolumn{6}{|l|}{ Neue Länder } \\
\hline Mitglieder* & 6.315 & 643 & 3.225 & 283 & 10.466 \\
\hline $\begin{array}{l}\text { Familien- } \\
\text { angehörige }\end{array}$ & 2.573 & 321 & 59 & 51 & 3.004 \\
\hline Versicherte & 8.888 & 964 & 3.284 & 334 & 13.470 \\
\hline $\begin{array}{l}\text { Familienan- } \\
\text { gehörige je } \\
\text { Mitglied }\end{array}$ & 0,41 & 0,50 & 0,02 & 0,18 & 0,29 \\
\hline
\end{tabular}

* Privatversicherte Mitglieder: Versicherte exkl. „mitversicherte“ Familienangehörige

** Summe aus GKV-Mitgliedern und PKV-Versicherte ohne Familienangehörige

Quelle: Bundesministerium für Arbeit und Sozialordnung (Hrsg.), Arbeitsund Sozialstatistik - Hauptergebnisse 1996, Bonn 1996, S. 204 und 209; Statistisches Bundesamt (Hrsg.), Versicherte in der Kranken- und Rentenversicherung 1995, Wiesbaden 1996

2 Streng genommen kann man unter den privat Versicherten nicht in der Form von "mitversicherten" Familienangehörigen wie in der gesetzlichen Krankenversicherung sprechen, da in der privaten Krankenversicherung für jede Person gesonderte Beiträge zu bezahlen sind, während die mitversicherten Familienangehörigen in der gesetzlichen Krankenversicherung beitragsfrei versichert sind. 
Seit Inkrafttreten des GRG könnten auch Arbeiter mit Einkommen über der Beitragsbemessungsgrenze die private Versicherung wählen. Allerdings ist der Anteil dieser Gruppe noch verschwindend klein, da sich der Wechsel nur für jüngere Leute lohnt und diese häufig nicht ausreichend hohe Einkommen beziehen.

Mit Blick auf eine Ausweitung oder Einschränkung des GKVVersichertenkreises ist die Zahl der freiwillig versicherten GKVMitglieder sowie der privat Versicherten (ohne mitversicherte Familienangehörige) von Interesse. Diese Gruppe umfaßt etwa 10,5 Mio. Personen in den alten und etwa 0,9 Mio. Personen in den neuen Ländern. Etwa die Hälfte dieser Personen sind $z$. Z. in der gesetzlichen Krankenversicherung, die andere Hälfte in der privaten Krankenversicherung versichert. Eine de facto Wahlmöglichkeit steht im Hinblick auf schlechte Risiken nicht allen GKV-Mitgliedern offen, und für alle PKV-Mitglieder besteht auch keine aktuelle Möglichkeit zum Wechsel.

Mindestens was die mitversicherten Familienangehörigen betrifft, wird aus den dargestellten Zahlen deutlich, daß eine Risikoselektion dieses Kreises zuungunsten der gesetzlichen Krankenversicherung erfolgt: Die Anzahl der mitversicherten Familienangehörigen je "Mitglied" ist in der privaten wesentlich niedriger als in der gesetzlichen Krankenversicherung. Die im Vergleich hohe Zahl der mitversicherten Familienangehörigen der freiwillig versicherten GKV-Mitglieder deutet darauf hin, daß gerade die unentgeltliche Mitversicherung einen Anreiz zur Wahl der gesetzlichen Krankenversicherung darstellt. Allerdings ist auch zu berücksichtigen, daß für Beamte, die im Durchschnitt mehr Kinder haben, ganz abgesehen von der Kinderzahl, die Beihilfe-Regelung die private Krankenversicherung kostengünstiger macht.

Die günstigere Versichertenselektion der privaten Krankenversicherung scheint $z$. Z. auch im Hinblick auf die Altersstruktur zu bestehen (vgl. Tabelle 2). Daraus wird vor allem ersichtlich, daß die privat Krankenversicherten zum überwiegenden Teil männlichen Geschlechts im Alter zwischen 30 und 60 Jahren und Bewohner der alten Bundesländer sind. Weibliche privat Versicherte sind weniger zahlreich, finden sich jedoch ebenfalls überwiegend in diesen Altersstufen. 
Tab. 2: Verteilung der Versicherten der gesetzlichen Krankenversicherung und privaten Krankenversicherung 1995 nach Alter und Geschlecht in 1000 für:

\begin{tabular}{|c|c|c|c|c|c|c|c|c|c|c|c|c|c|}
\hline \multicolumn{7}{|c|}{ Alte Bundesländer } & \multicolumn{7}{|c|}{\begin{tabular}{||c|} 
Neue Bundesländer \\
\end{tabular}} \\
\hline Mănner & $\begin{array}{l}\text { Pflicht- } \\
\text { mitgl. }\end{array}$ & \begin{tabular}{|l} 
Freiw. \\
Mitgl.
\end{tabular} & $\begin{array}{l}\text { KVdR } \\
\text {-Mitgl. }\end{array}$ & \begin{tabular}{|c|} 
GKV- \\
Fam.an \\
\end{tabular} & $\begin{array}{l}\text { PKV- } \\
\text { Vers. }\end{array}$ & \begin{tabular}{|c|} 
PKV- \\
Fam.an
\end{tabular} & Männer & $\begin{array}{l}\text { Pflicht- } \\
\text { mitgl. }\end{array}$ & $\begin{array}{l}\text { Freiw. } \\
\text { Mitgl. }\end{array}$ & $\begin{array}{l}\text { KVdR } \\
\text {-Mitgl. }\end{array}$ & $\begin{array}{c}\text { GKV- } \\
\text { Fam.an }\end{array}$ & $\begin{array}{l}\text { PKV- } \\
\text { Vers. }\end{array}$ & $\begin{array}{l}\text { PKV- } \\
\text { Fam.a }\end{array}$ \\
\hline$>\mathrm{r} 65$ & 25 & 150 & 3.318 & 108 & 380 & 1 & $>65$ & 5 & 1 & 777 & 26 & 1 & 1 \\
\hline $60-64$ & 313 & 199 & 999 & 59 & 174 & 1 & $60-64$ & 113 & 18 & 288 & 25 & 1 & 7 \\
\hline $50-59$ & 2.549 & 853 & 449 & 129 & 736 & I & $50-59$ & 877 & 91 & 92 & 35 & 23 & 1 \\
\hline $40-49$ & 2.453 & 764 & 63 & 66 & 869 & 1 & $40-49$ & 813 & 93 & 24 & 11 & 53 & 1 \\
\hline $30-39$ & 3.484 & 770 & 48 & 100 & 757 & 5 & $30-39$ & 1.060 & 84 & 27 & 10 & 76 & 1 \\
\hline $20-29$ & 3.123 & 284 & 1 & 573 & 282 & 76 & $20-29$ & 760 & 30 & 1 & 85 & 30 & 1 \\
\hline $15-19$ & 515 & 15 & 1 & 960 & 67 & 110 & $15-20$ & 186 & 1 & 1 & 301 & 1 & 6 \\
\hline$<15$ & 1 & 37 & 1 & 4.612 & 191 & 395 & $<15$ & 1 & 3 & 1 & 1.285 & 7 & 18 \\
\hline Alle & $\begin{array}{r}12.46 \\
0\end{array}$ & 3072 & 4.878 & 6.601 & 3456 & 593 & Alle & 3.815 & 323 & 1208 & 1.776 & 194 & 25 \\
\hline Frauen & $\begin{array}{l}\text { Pflicht- } \\
\text { mitgl. }\end{array}$ & $\begin{array}{l}\text { Freiw. } \\
\text { Mitgl. }\end{array}$ & $\begin{array}{l}\text { KVdR } \\
\text {-Mitgl. }\end{array}$ & \begin{tabular}{|c|} 
GKV- \\
Fam.an
\end{tabular} & $\begin{array}{l}\text { PKV- } \\
\text { Vers. }\end{array}$ & $\begin{array}{l}\text { PKV- } \\
\text { Fam.an }\end{array}$ & Frauen & $\begin{array}{l}\text { Pflicht- } \\
\text { mitgl. }\end{array}$ & $\begin{array}{l}\text { Freiw. } \\
\text { Mitgl. }\end{array}$ & \begin{tabular}{|c|} 
KVdR \\
-Mitgl.
\end{tabular} & $\begin{array}{l}\text { GKV- } \\
\text { Fam.an }\end{array}$ & \begin{tabular}{|l|} 
PKV- \\
Vers.
\end{tabular} & $\begin{array}{l}\text { PKV- } \\
\text { Fam.a }\end{array}$ \\
\hline$>65$ & 21 & 111 & 5.353 & 841 & 323 & 64 & $>65$ & 1 & 1 & 1.459 & 55 & 1 & 1 \\
\hline $60-64$ & 117 & 58 & 881 & 654 & 71 & 30 & $60-64$ & 15 & 1 & 458 & 21 & 1 & 1 \\
\hline 50-59 & 1.897 & 254 & 378 & 1.646 & 320 & 112 & 50-59 & 869 & 38 & 142 & 112 & 8 & 1 \\
\hline $40-49$ & 2.192 & 249 & 70 & 1.165 & 434 & 105 & $40-49$ & 825 & 56 & 27 & 48 & 23 & 1 \\
\hline $30-39$ & 2.546 & 331 & 50 & 1.572 & 396 & 101 & $30-39$ & 1.044 & 48 & 25 & 61 & 28 & 1 \\
\hline $20-29$ & 2.754 & 217 & 1 & 1.090 & 230 & 75 & $20-29$ & 750 & 24 & 1 & 96 & 18 & 1 \\
\hline $15-19$ & 387 & 17 & 1 & 996 & 60 & 103 & $15-19$ & 127 & 6 & 1 & 319 & 1 & 1 \\
\hline$<15$ & 1 & 32 & 1 & 4.313 & 183 & 385 & $<15$ & 1 & 3 & 1 & 1.204 & 6 & 16 \\
\hline Alle & 9.915 & 1.271 & 6.732 & 12.283 & 2.026 & 974 & Alle & 3.629 & 174 & 2.111 & 1.907 & 88 & 25 \\
\hline
\end{tabular}

/ nicht nachgewiesen bzw. nicht besetzt

Quelle: Eigene Darstellung nach Statistisches Bundesamt (Hrsg.), Versicherte in der Kranken- und Rentenversicherung 1995, Wiesbaden 1996, S. $52 \mathrm{ff}$. und $88 \mathrm{ff}$.

Die geringe Zahl älterer privat Versicherter ist mindestens noch zum Teil direkte Konsequenz der früheren Pflichtversicherung der Rentner in der KVdR. Mit dem Gesundheitsreformgesetz (GRG) wurde allerdings die KVdR-Mitgliedschaft an sehr weitgehende Vorversicherungszeiten in der GKV geknüpft. Mit dem Gesundheitsstrukturgesetz (GSG) wurden diese Regelungen noch einmal verschärft, so daß nur langjährig Pflichtversicherte in Zukunft KVdR-Mitglieder sein werden ${ }^{3}$. Diese Veränderung in der KVdR-

3 Durch das GRG wurde den privat Versicherten der Weg in die KVdR in den meisten Fällen praktisch versperrt. Nach § 5 [1] Nr. 11 SGB V (in der derzeit geltenden Fassung des GSG) ist Voraussetzung für die KVdRMitgliedschaft, daß neun Zehntel der zweiten Hälfte der Periode zwischen Aufnahme der ersten Erwerbstätigkeit und Rentenantrag Pflichtmitgliedschaft in der GKV bestanden hat, wobei der Status als mitversicherte(r) Familienangehörige( $r$ ) eines Pflichtmitglieds gleich berücksichtigt wird. Die 
Versicherungspflicht wird längerfristig zu einer veränderten Altersstruktur der Versicherten der privaten Krankenversicherung führen (vgl. Abbildung 2).

Abb. 2

Geschătzte Entwicklung der Verteilung der GKV-Versicherten in Deutschland auf Basis der 8. koordinierten BVS (mittlere Variante) 1994 -

2040Vergleich der Entwicklung dynamisches Modell und statisches Modell

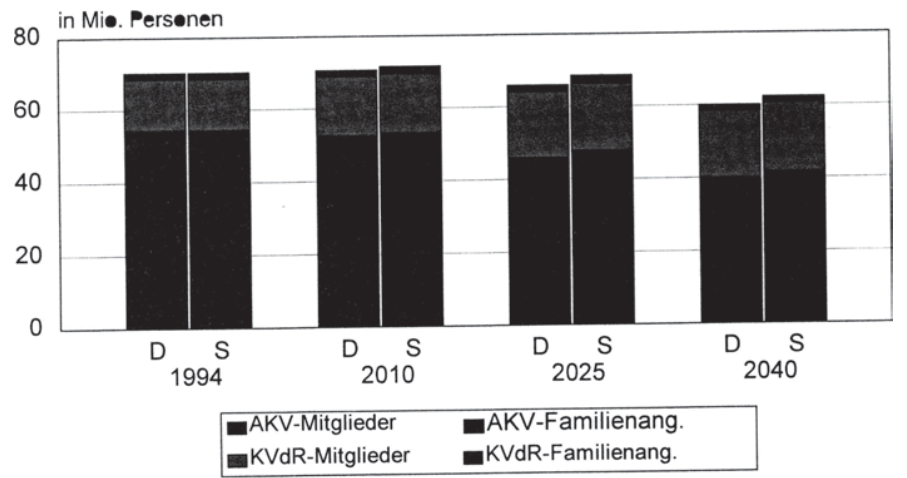

D: dynamisches Modell, keine Rückkehr alterer Versicherter von der PKV in die GKV

S: statisches Modell, altersspezifisch konstante GKV-Anteile an der Bevölkerung

Quelle: INIFES 1996

Die Rentner mit langjähriger privater Krankenversicherung werden in zunehmendem Maß in der privaten Krankenversicherung verbleiben, was zu einer Altersverschiebung in dieser Gruppe führen wird und $\mathrm{zu}$ einem geringeren Zuwachs der KVdRMitglieder in der gesetzlichen Krankenversicherung. In Abbildung 2 wird die (auf der Basis der 8. koordinierten Bevölkerungsvorausberechnung) voraussichtlich zu erwartende Entwicklung der GKV-Versichertenzahlen auf der Basis zweier verschiedener Annahmen dargestellt.

Die Variante $\mathrm{S}$ geht davon aus, daß in den einzelnen Alters- und Geschlechtsgruppen der Anteil der GKV-Versichertengruppen konstant bleibt. In der Variante $D$ wird unterstellt, daß der Anteil der KVdR-Mitglieder (und KVdR-Familienangehörigen) abnimmt.

Regelung wurde durch das GSG weiter verschärft, indem entsprechende Vorversicherungszeiten als freiwillig GKV-Versicherte nicht zur Versicherungspflicht in der KVdR führen, sondern nur zur weiteren freiwilligen Versicherung berechtigen. Wegen der für freiwillig Versicherte möglicherweise größeren Beitragsbemessungsgrundlage kann dies zu einer höheren Beitragsbelastung dieses Personenkreises führen. 
Diese Annahmen führen entsprechend zu geringeren Versichertenzahlen in der gesetzlichen Krankenversicherung bei unterstellt gleicher Bevölkerungsentwicklung.

Die Versichertenstruktur der gesetzlichen Krankenversicherung beeinflußt in nicht unerheblichem Maß ihre Einnahmen. Aus Abbildung 3 wird ersichtlich, wie sich in den alten und den neuen Ländern im Jahr 1995 das Beitragsaufkommen verteilt hat.

Abb. 3: Verteilung des Beitragsaufkommens der gesetzlichen Krankenversicherung auf Mitgliedergruppen, Deutschland 1995
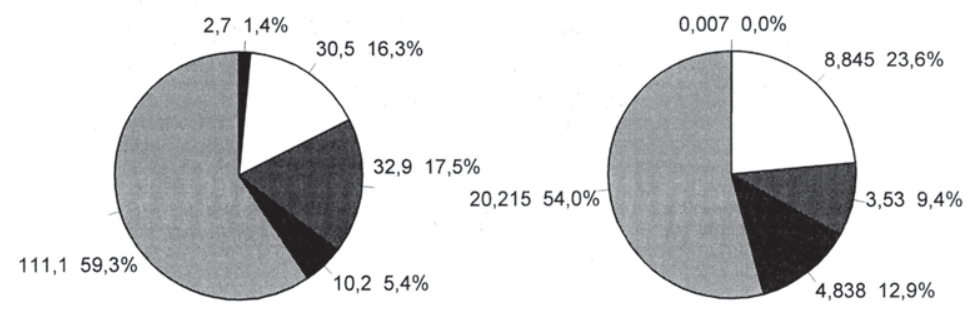

$\square$ AKV-Pflichtmitg

AKV-Arbeitslose $\square$ AKV-Freiw. Mitg.

$\square$ KVdR-Renten (GRV)

KVdR-Sonstige

Quelle: Bundesministerium für Arbeit und Sozialordnung (1997), Arbeitsund Sozialstatistik - Hauptergebnisse 1997, Bonn, S. 163 und 171

Der überwiegende Teil der GKV-Beitragseinnahmen wird von Pflichtmitgliedern aufgebracht. In zunehmendem Maß wird jedoch die Arbeitslosigkeit für die angespannte Finanzlage der Sozialversicherungen allgemein und der gesetzlichen Krankenversicherung im besonderen verantwortlich gemacht. Dabei wirkt sich die hohe Arbeitslosigkeit in mehrfacher Hinsicht aus. In den letzten Jahren ist zum einen der Anteil der Leistungsempfänger der Bundesanstalt für Arbeit an der steigenden Zahl der Arbeitslosen stagnierend bis rückläufig. Da nur für Leistungsempfänger der Bundesanstalt für Arbeit Beiträge an die anderen Sozialversicherungszweige gezahlt werden, beeinträchtigt dies auch die Finanzlage der gesetzlichen Krankenversicherung negativ. Zum anderen wird neuerdings nicht das Einkommen vor Eintritt der Arbeitslosigkeit, sondern nur die Lohnersatzleistung als Bemessungsgrundlage der Krankenversicherungsbeiträge herangezogen. 
Auch das reduziert künftig die Einnahmen der Krankenkassen für Arbeitslose.

Um die quantitative Bedeutung der Arbeitslosenbeiträge zu verdeutlichen, werden sie in Abbildung 3 gesondert von anderen Pflichtbeiträgen der allgemeinen Krankenversicherung (AKV) ausgewiesen. In den alten Bundesländern machten sie im Jahr 1995 bereits $5,4 \%$, in den neuen Bundesländern sogar 12,9\% aus. Die durchschnittlichen Beiträge der freiwillig versicherten Mitglieder sind zwar sicher nicht alle sehr hoch - die Verteilung der Beiträge der Selbständigen dürfte eher breit streuen -, dennoch sind mindestens die Angestellten mit Einkommen über der Bemessungsgrenze Spitzenbeitragszahler. Der Beitrag der freiwillig Versicherten ist deshalb auch überproportional: Die 12,4\% freiwillig versicherten Mitglieder bringen $17,5 \%$ des Beitragsvolumens der gesetzlichen Krankenversicherung in den alten Bundesländern auf. Ein Verlust solcher Mitglieder wäre deshalb für die gesetzliche Krankenversicherung sicher von Nachteil, da sie mindestens hinsichtlich ihrer Beitragseinnahmen "gute Risiken" darstellen. Andererseits ist davon auszugehen, daß ein Zugewinn an weiteren Angestellten mit Einkommen über der Beitragsbemessungsgrenze nicht nur einen überproportionalen Beitragszuwachs, sondern auch vermutlich eher unterproportionale Ausgabenzuwächse bringen könnte.

\section{Auswirkungen von Finanzierungsalternativen}

Im folgenden werden Modellrechnungen für einige Finanzierungsalternativen der gesetzlichen Rentenversicherung vorgestellt. Dabei wurden die Angaben für das Jahr 1994 berechnet ${ }^{4}$. Da für die neuen Bundesländer teils Daten fehlen oder die Alternativen von nur geringer Bedeutung sind, beschränkt sich die Darstellung auf die alten Länder. Im wesentlichen werden drei Gruppen unterschieden:

- Ausweitung des Versichertenkreises (mit teilweiser Anhebung der Beitragsbemessungsgrenze),

- Ausweitung des beitragspflichtigen Einkommens (Bemessungsgrundlage) bei Beitragspflicht für die Gesamtbevölkerung und Reform der Familienhilfe,

4 Die Rechnungsergebnisse beruhen auf einem von der Hans-BöcklerStiftung geförderten Projekt (vgl. Busch/Pfaff/Rindsfüßer 1996). 
- Ausweitung des Versichertenkreises.

Drei Alternativen der Ausweitung des Versichertenkreises werden untersucht:

Modell V1: Anhebung der Beitragsbemessungs- und der Versicherungspflichtgrenze für Arbeitnehmer auf die der Beitragsbemessungsgrenze der Rentenversicherung der Arbeiter und Angestellten.

Model V2: Totaler Wegfall der Versicherungspflichtgrenze mit Anhebung der Beitragsbemessungsgrenze auf die der Beitragsbemessungsgrenze der Rentenversicherung der Arbeiter und Angestellten.

Modell V3: Ausweitung der Versicherungspflicht in die gesetzliche Krankenversicherung auf alle privat Versicherten (Beibehaltung der Sondersysteme).

Wie diese Alternativen sich auf die Versichertenzahl, die Ausgaben und die Einnahmen der gesetzlichen Krankenversicherung in den alten Ländern auswirken würden, ist in Tabelle 3 zusammengefaßt: 
Tab. 3: Auswirkung einer Ausweitung der Versicherungspflicht auf Versichertenzahl, Ausgaben, Einnahmen und Beitragssätze der gesetzlichen Krankenversicherung in den alten Ländern, Rechnungsbasis 1994

\begin{tabular}{|c|c|c|c|}
\hline $\begin{array}{l}\text { Modelle } \\
\text { Auswirkung }\end{array}$ & $\begin{array}{c}\text { Modell V1 } \\
\text { Anhebung der VPG und } \\
\text { der BBG auf die der GRV } \\
\text { für Arbeiter und Angestell- } \\
\text { te }\end{array}$ & $\begin{array}{l}\text { Modell V2 } \\
\text { Wegfall der VPG und } \\
\text { Anhebung der BBG auf } \\
\text { die der GRV für Arbeiter } \\
\text { und Angestellte }\end{array}$ & $\begin{array}{c}\text { Modell V3 } \\
\text { Beitragspflicht aller } \\
\text { privat Versicherten in der } \\
\text { GKV }\end{array}$ \\
\hline $\begin{array}{l}\text { Versicherte: } \\
\text { in Mio. } \\
\text { (in \%) }\end{array}$ & $\begin{array}{r}56,81 \\
(+1,6 \%) \\
\end{array}$ & $\begin{array}{r}57,16 \\
(+2,2 \%) \\
\end{array}$ & $\begin{array}{r}62,98 \\
(+12,6 \%) \\
\end{array}$ \\
\hline $\begin{array}{l}\text { Ausgaben: } \\
\text { in Mrd. DM } \\
\text { (in \%) }\end{array}$ & $\begin{array}{r}180,25 \\
(+1,7 \%)\end{array}$ & $\begin{array}{r}181,33 \\
(+2,3 \%)\end{array}$ & $\begin{array}{r}197,59 \\
(+11,5 \%) \\
\end{array}$ \\
\hline $\begin{array}{l}\text { Einnahmen*: } \\
\text { in Mrd. DM } \\
\text { (in \%) }\end{array}$ & $\begin{array}{r}190,12 \\
(+7,3 \%) \\
\end{array}$ & $\begin{array}{r}193,19 \\
(+9,0 \%) \\
\end{array}$ & $\begin{array}{r}206,00 \\
(+16,4 \%) \\
\end{array}$ \\
\hline $\begin{array}{l}\text { Beitrags- } \\
\text { satz }{ }^{\star *} \text { : in } \% \\
\text { (in } \% \text {-Pkt.) }\end{array}$ & $\begin{array}{r}12,22 \% \\
(-0,67 \%-P k t .)\end{array}$ & $\begin{array}{r}12,10 \% \\
(-0,79 \% \text {-Pkt. })\end{array}$ & $\begin{array}{r}12,35 \% \\
(-0,54 \% \text {-Pkt. })\end{array}$ \\
\hline
\end{tabular}

BBG - Beitragsbemessungsgrenze; VPG - Versicherungspflichtgrenze; GRV - gesetzliche Rentenversicherung

${ }^{*}$ Einnahmen bei unverändertem Beitragssatz

** rechnerisch kostendeckender Beitragssatz

Quelle: Busch/Pfaff/Rindfüßer 1996, S. 50, 53, 57

Im Modell V1 steigen die Versichertenzahlen, da bislang in der privaten Krankenversicherung oder gar nicht versicherte Angestellte und Arbeiter mit Einkommen über der Versicherungspflichtgrenze unter die Versicherungspflicht fallen würden. Selbstverständlich kommen für diese Mitglieder sowie ihre mitversicherten Familienangehörigen zusätzliche Ausgaben hinzu. Diese werden jedoch von den durch sie hinzukommenden Beitragseinnahmen überwogen, die in Tabelle 3 zunächst mit dem bisher kostendeckenden rechnerischen Beitragssatz ${ }^{5}$ berechnet wurden. Die Einnahmen steigen jedoch außerdem, weil bisher freiwillig versicherte Angestellte und Arbeiter nunmehr in aller Regel höhere Beiträge bezahlen müßten. Diesen Beitragsmehreinnahmen

5 Als Referenzbeitragssatz wurde jener Satz herangezogen, der die Leistungsausgaben voll gedeckt hätte, ohne Überschüsse oder Defizite zu verursachen. Der tatsächliche Beitragssatz weicht in der Regel etwas davon ab, da er auch die Verwaltungsausgaben abdecken muß und eine genaue Vorausberechnung oft schwierig ist. 
stehen jedoch nur beim Krankengeld etwas höhere Ausgaben gegenüber.

Per Saldo hätte diese Maßnahme im Durchschnitt der Krankenkassen eine Beitragssatzsenkung um 0,67 Beitragssatzpunkte gestattet.

Würde man gemäß Modell V2 die Beitragsbemessungsgrenze der Rentenversicherung der Arbeiter und Angestellten beibehalten, für Angestellte und Arbeiter - analog zur gesetzlichen Rentenversicherung - jedoch die Versicherungspflichtgrenze gänzlich aufheben, so wäre bei einer Entwicklung, wie in Spalte 2 der Tabelle 1 dargestellt, eine Beitragssatzminderung von durchschnittlich 0,79 Beitragssatzpunkten zu realisieren.

Die in Modell V3 simulierte Eingliederung aller PKV-Versicherten würde zwar einen deutlich größeren Zuwachs an Versicherten bringen als die beiden anderen Modelle, es muß jedoch unterstellt werden, daß die zusätzlich Versicherten nicht nur "gute Risiken" hinsichtlich ihrer Relation der beitragspflichtigen Einkommen und ihrer Ausgabenstruktur sind. In der Rechnung wurde unterstellt, daß die beitragspflichtigen Einkommen alters- und geschlechtsspezifisch gleich verteilt wären wie bei den bisherigen GKV-Mitgliedern. Dies scheint für Beamte nicht unrealistisch zu sein. Auch bei den Selbständigen ist nicht anzunehmen, daß nur Höchstverdiener hinzukommen würden.

Unter diesen - zugegebenerweise konservativen - Annahmen würde eine nur um 0,54 Beitragssatzpunkte geringere Beitragssatzentlastung zustande kommen.

\section{Wahl der Bemessungsgrundlage}

Um die Auswirkung einer unterschiedlichen Abgrenzung der Bemessungsgrundlage, $d . h$. der beitragspflichtigen Einnahmen zu umreißen, wurde einheitlich von einer die gesamte Wohnbevölkerung umfassenden Beitragspflicht ausgegangen. Diese Annahme führt zu einem in allen Modellen im Vergleich zum Status quo um $18,7 \%$ größeren Versichertenkreis.

Unterstellt man - wie im Modell B1 - eine identische Struktur der beitragspflichtigen Einkommen und der Leistungsausgaben wie bei den bisher in der gesetzlichen Krankenversicherung Versi- 
cherten, so bringt eine Ausweitung der Versicherungspflicht auf die Wohnbevölkerung in etwa ausgewogene Zuwächse von Einnahmen und Ausgaben und ermöglicht deshalb unter diesen Annahmen eine nur geringe Beitragsentlastung von 0,18 Beitragssatzpunkten (vgl. Tabelle 4).

Tab. 4: Auswirkung einer allgemeinen Versicherungspflicht bei verschiedener Beitragsbemessungsgrundlage auf Versichertenzahl, Ausgaben, Einnahmen und Beitragssätze der gesetzlichen Krankenversicherung in den alten Ländern, Rechnungsbasis 1994

\begin{tabular}{|c|c|c|c|}
\hline $\begin{array}{l}\text { Modelle } \\
\text { Auswirkung }\end{array}$ & $\begin{array}{l}\text { Modell B1 } \\
\text { Ausgaben und Ein- } \\
\text { nahmenstruktur wie bis- } \\
\text { herige GKV-Versicherte }\end{array}$ & $\begin{array}{c}\text { Modell B2 } \\
\text { Beitragsbelastung aller } \\
\text { Arbeitseinkommen, } \\
\text { Wegfall der BBG }\end{array}$ & $\begin{array}{c}\text { Modell B3 } \\
\text { Beitragsbelastung des } \\
\text { gesamten Volksein- } \\
\text { kommens, der Renten } \\
\text { und der BA-Leistungen, } \\
\text { Wegfall der BBG }\end{array}$ \\
\hline \multirow{4}{*}{$\begin{array}{l}\text { Versicherte: } \\
\text { in Mio. } \\
\text { (in \%) } \\
\text { Ausgaben: } \\
\text { in Mrd. DM } \\
\text { (in \%) } \\
\text { Einnahmen*: } \\
\text { in Mrd. DM } \\
\text { (in \%) } \\
\text { Beitrags- } \\
\text { satz*: in \% } \\
\text { (in \%-Pkt.) }\end{array}$} & $\begin{array}{c}66,41 \\
(+18,7 \%) \\
\end{array}$ & $\begin{array}{c}66,41 \\
(+18,7 \%) \\
\end{array}$ & $\begin{array}{c}66,41 \\
(+18,7 \%) \\
\end{array}$ \\
\hline & $\begin{array}{c}207,58 \\
(+17,1 \%)\end{array}$ & $\begin{array}{c}209,35 \\
(+18,1 \%) \\
\end{array}$ & $\begin{array}{c}209,35 \\
(+18,1 \%) \\
\end{array}$ \\
\hline & $\begin{array}{l}210,44 \\
(+18,7 \%)\end{array}$ & $\begin{array}{c}234,34 \\
(+32,2 \%)\end{array}$ & $\begin{array}{c}328,73 \\
(+85,5 \%)\end{array}$ \\
\hline & $\begin{array}{l}12,71 \% \\
(-0,18 \% \text {-Pkt.) }\end{array}$ & $\begin{array}{l}11,52 \% \\
(-1,37 \% \text {-Pkt.) }\end{array}$ & $\begin{array}{c}8,21 \% \\
\text { (-4,68 \%-Pkt.) }\end{array}$ \\
\hline
\end{tabular}

BBG - Beitragsbemessungsgrenze; BA-Leistungen - Leistungen der Bundesanstalt für Arbeit

* Einnahmen bei unverändertem Beitragssatz

** rechnerisch kostendeckender Beitragssatz

Quelle: Busch/Pfaff/Rindfüßer 1996, S. 59, 60, 64

Modell B2 geht von einer deutlichen Ausweitung der beitragspflichtigen Einkommen auf alle Arbeitseinkommen inkl. rechnerischer Arbeitseinkommen der Selbständigen aus. Die Bemessungsgrenze wird in dieser Variante aufgehoben. Dies würde zu geringfügigen Ausgabensteigerungen beim Krankengeld führen. Die Finanzkraft würde dagegen deutlich gesteigert, so daß dies eine Minderung des Beitragssatzes um 1,37 Beitragssatzpunkte ermöglichen würde.

Modell B3 ist zwar sicher unrealistisch, es wurde jedoch mit aufgenommen, um den rechnerisch maximalen Beitragssatzeffekt zu 
ermitteln. Dabei wird davon ausgegangen, daß alle primären Einkommen ohne Beitragsbemessungsgrenze beitragspflichtig würden. Daneben wird - wie im jetzigen System - unterstellt, daß auch die aus der staatlichen Umverteilung finanzierten Renten und Leistungen der Bundesanstalt für Arbeit (wie auch z. Z. quasi noch einmal nach der Umverteilung) beitragsbelastet werden. Rechnerisch würde dies eine Beitragsentlastung von 4,68 Beitragssatzpunkten ermöglichen.

\section{Reform der Familienhilfe}

In Tabelle 5 werden Reformalternativen der Familienhilfe untersucht. Dabei setzt Modell $K$ an der Finanzierung der Leistungsausgaben für Kinder an, die Modelle F1 und F2 bei der Erhebung eigener Beiträge für bislang beitragsfrei mitversicherter Ehepartner(innen).

Tab. 5: Auswirkung einer Reform der Familienhilfe auf Versichertenzahl, Ausgaben, Einnahmen und Beitragssätze der gesetzlichen Krankenversicherung in den alten Ländern, Rechnungsbasis 1994

\begin{tabular}{|c|c|c|c|}
\hline $\begin{array}{l}\text { Modelle } \\
\text { Auswirkung }\end{array}$ & $\begin{array}{l}\text { Modell K } \\
\text { Ausgliederung (und Steuer- } \\
\text { finanzierung) der Kosten } \\
\text { von bislang beitragsfrei } \\
\text { versicherten Kindern }\end{array}$ & $\begin{array}{c}\text { Modell F1 } \\
\text { Beitragsleistung für } \\
\text { Ehepartner, die keine } \\
\text { Kinder unter } 7 \text { Jahren } \\
\text { erziehen ( } 50 \% \text { des } \\
\text { Mitgliedsbeitrags) }\end{array}$ & $\begin{array}{l}\text { Modell F2 } \\
\text { Beitragsleistung für alle } \\
\text { (bislang beitragsfrei } \\
\text { mitversicherten) Ehe- } \\
\text { partner ( } 50 \% \text { des Mit- } \\
\text { gliedsbeitrags) } \\
\end{array}$ \\
\hline \multirow{4}{*}{$\begin{array}{l}\text { Versicherte: in Mio. } \\
\text { (in \%) } \\
\text { Ausgaben: in Mrd. DM } \\
\text { (in \%) } \\
\text { Einnahmen*: } \\
\text { in Mrd. DM } \\
\text { (in \%) } \\
\text { Beitrags-satz**: in \% } \\
\text { (in \%-Pkt.) }\end{array}$} & $\begin{array}{r}45,14 \\
(-19,3 \%) \\
\end{array}$ & $\begin{array}{r}55,93 \\
( \pm 0 \%) \\
\end{array}$ & $\begin{array}{r}55,93 \\
( \pm 0 \%) \\
\end{array}$ \\
\hline & $\begin{array}{l}165,205 \\
(-6,8 \% \\
\end{array}$ & $\begin{array}{l}177,24 \\
(+0 \%) \\
\end{array}$ & $\begin{array}{l}177,24 \\
(+0 \%) \\
\end{array}$ \\
\hline & $\begin{array}{r}176,51 \\
(-0,4 \%) \\
\end{array}$ & $\begin{array}{r}190,26 \\
(+7,3 \%) \\
\end{array}$ & $\begin{array}{r}195,40 \\
(+10,2 \%) \\
\end{array}$ \\
\hline & $\begin{array}{r}12,06 \% \\
(-0,83 \%-P k t .) \\
\end{array}$ & $\begin{array}{r}12,01 \% \\
(-0,88 \%-\text { Pkt. }) \\
\end{array}$ & $\begin{array}{r}11,69 \% \\
(-1,20 \%-P k t .) \\
\end{array}$ \\
\hline
\end{tabular}

* Einnahmen bei unverändertem Beitragssatz

** rechnerisch kostendeckender Beitragssatz

Quelle: Busch/Pfaff/Rindfüßer 1996, S. 72, 73, 75

In Modell $K$ wird untersucht, in welchem Maß eine Steuerfinanzierung von Leistungsausgaben für Kinder und Jugendliche bis 18 Jahre die Versichertenzahl und die Leistungsausgaben reduzieren würde. Nachdem auch für bislang bereits pflichtversicherte Jugendliche eine beitragsfreie Versicherung vorgesehen würde, wäre auch eine - allerdings sehr geringe - Reduzierung der Beitragseinnahmen impliziert. Die zwar deutliche Reduzierung der Versichertenzahlen um fast ein Fünftel wäre angesichts der im 
Durchschnitt niedrigen Leistungsausgaben für Kinder und Jugendliche allerdings nur von einem im Vergleich geringen Rückgang der Ausgaben von etwa 6,8 \% begleitet. Insgesamt wäre es möglich, die Beiträge dadurch immerhin um 0,86 Beitragssatzpunkte abzusenken. In diesem Zusammenhang soll allerdings ganz deutlich gemacht werden, daß es keinesfalls darum gehen sollte, die Leistungen für Kinder und Jugendliche zu streichen. Vielmehr sollten sie als gesamtgesellschaftliche Aufgabe aus Steuermitteln finanziert werden. Wäre dies der Fall, müßte die Regelung logischerweise auch auf bislang privat versicherte Kinder und Jugendliche ausgedehnt werden.

Sieht man wie in den Modellen F1 und F2 eine eigene Beitragsleistung für bisher beitragsfrei mitversicherte Ehepartner(innen) vor, so muß entschieden werden, ob dies für alle oder nur für nicht (kleine) Kinder Erziehende gelten soll. In Modell F1 wurde unterstellt, die Ehepartner(innen), die Kinder bis zu sechs Jahren erziehen und nicht mehr als geringfügig beschäftigt sind, wären wie bisher beitragsfrei mitversichert. Modell F2 geht von einer generellen Beitragspflicht für alle Ehepartner(innen) aus. Des weiteren muß eine Norm für die (unterstellten) beitragspflichtigen Einkommen gewählt werden. Als Bandbreite hierfür ist als Untergrenze an ein Einkommen an der unteren Versicherungspflichtgrenze (1997: $610 \mathrm{DM}$ in den alten Ländern) zu denken. Als realistische Obergrenze könnte das halbe Einkommen des Mitglieds unterstellt werden. Letztere Norm wurde in den vorliegenden Rechnungen zugrunde gelegt.

Die Annahmen der Modelle F1 und F2 führen zu keiner Ausweitung des Versichertenkreises und der Leistungsausgaben, wohl aber zu einer Verbreiterung der Beitragsbemessung und somit zu einer möglichen Reduzierung des Beitragssatzes um 0,88 bzw. um 1,20 (bei genereller Beitragspflicht) Beitragssatzpunkte. Neben dem negativen Effekt der Belastung auch von Familien mit Kindern hätten die Modelle F1 und F2 den Anreiz zur Erwerbstätigkeit, bzw. sie würden Schwarzarbeit weniger attraktiv machen.

\section{Zum Abschluß}

Die untersuchten Finanzierungsoptionen weisen eine nicht unerhebliche Bandbreite von Effekten auf. In den vorliegenden Ergebnissen wurden gleichsam „Endeffekte" dargestellt. Es wurde nicht darauf eingegangen, wie sich der Übergang gestalten 
könnte. Insbesondere wurde darauf verzichtet zu untersuchen, ob aufgrund eines "Vertrauensschutzes", etwa bei der Ausweitung von Versicherungspflicht, bestimmten Gruppen ein "Bleiberecht" in der alten Versicherung eingeräumt werden müßte und somit die aufgeführten Effekte erst längerfristig auftreten könnten.

Schließlich muß im Kontext einer tatsächlichen Reform sicher auch die Leistungsseite betrachtet werden. Da die simultane Veränderung auf Finanzierungs- und Leistungsseite eventuell gegenläufige Effekte enthalten kann, bietet sich zur Beurteilung einzelner Maßnahmen dennoch eine isolierte Betrachtung an.

\section{Literatur}

Bundesministerium für Arbeit und Sozialordnung (Hrsg.), Arbeits- und Sozialstatistik Hauptergebnisse 1996, Bonn 1996

Bundesministerium für Arbeit und Sozialordnung (Hrsg.), Arbeits- und Sozialstatistik Hauptergebnisse 1997, Bonn 1997

Busch, Susanne, Anita B. Pfaff, und Christian Rindsfüßer: Die Finanzierung der gesetzlichen Krankenversicherung - Möglichkeiten zur Umgestaltung und Ergebnisse ausgewählter Modellrechnungen, Graue Reihe der Hans Böckler Stiftung, Bd. 110, Düsseldorf 1996

SVRKAiG (Sachverständigenrat für die Konzertierte Aktion im Gesundheitswesen), Gesundheitswesen in Deutschland: Kostenfaktor und Zukunftsbranche, Band II Fortschritt und Wachstumsmärkte, Finanzierung und Vergütung, Sondergutachten 1997 Kurzfassung, Bonn 1997

Statistisches Bundesamt (Hrsg.), Versicherte in der Kranken- und Rentenversicherung 1995, Fachserie 13, Reihe 1, Wiesbaden 1996 


\section{Einnahmenentwicklung der GKV und Finanzierungsoptionen}

Frank E. Münnichs

Was ich zu sagen habe, werden Sie zum großen Teil schon gehört haben. Wir sind hier ja alle Experten. Wir kennen die Probleme. Wir haben die ausführlichen Berichte des Sachverständigenrats und die Untersuchung von Frau Kollegin Pfaff gelesen. Wir haben hervorragende Referate gehört. Also: erwarten Sie jetzt bitte nichts wesentlich Neues.

Im Jahre 1992 hat die angesehene angelsächsische Wirtschaftsberatungsgesellschaft National Economic Research Associates ${ }^{7}$ die voraussichtlichen Finanzierungsdefizite der Systeme der sozialen Sicherheit auf dem Gesundheitssektor für 16 Staaten auf der Basis 1990/1991 geschätzt. Das Schaubild 1 zeigt die Ergebnisse dieser Berechnungen, extrapoliert auf das Jahr 2000.

Abb. 1

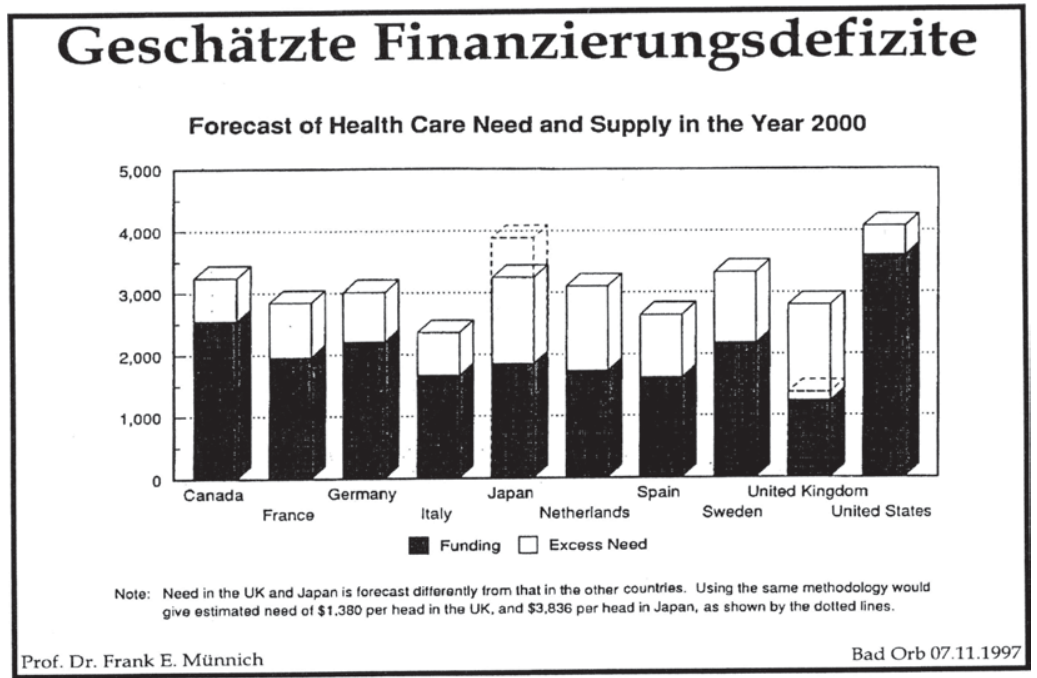

6 Überarbeitete Fassung eines Impulsreferats, gehalten auf den 2. Bad Orber Gesprächen über kontroverse Themen im Gesundheitswesen. Bad Orb. 7. - 8. November 1997.

7 National Economic Research Associates (n/e/r/a), Gutachten für die Pharmaceutical Partners for Better Health Care. $1992 \mathrm{ff}$. 
Der generelle Eindruck ist, daß, unabhängig von der Art oder Struktur der Sozialversicherungssysteme, die in den einzelnen Ländern existieren, unabhängig von den Regierungen, die dort an der Macht sind, und unabhängig von den jeweiligen medizinischen Schulen, ein Finanzierungsdefizit zu erwarten ist. Diese Finanzierungsdefizite wurden mit recht brutalen ökonometrischen Verfahren ermittelt. Sie dürften aber dennoch einen guten Eindruck von dem vermitteln, was diese Länder zu erwarten haben. Der Gesundheitssektor ist eben aus einer Reihe von Gründen ein Wachstumssektor per se.

Die Entwicklungen im Gesundheitswesen, die gegenwärtig zu diesen Finanzierungsdefiziten führen, sind nach meiner Überzeugung aber nur zum Teil endogen bedingt. Die deutsche Volkswirtschaft, die alle diese Leistungen trägt und aus deren Produktivität diese Leistungen erbracht werden, steht, wie ihre westeuropäischen Schwestern, in einem weltweiten wirtschaftlichen, technischen und kulturellen Wettbewerb, der über die innere Dynamik des Gesundheitswesens hinaus weitere mächtige Triebkräfte auslöst, die sich auf die Finanzierung der Gesundheitssysteme auswirken und die eine weitere Öffnung der Schere zwischen Anspruch und Möglichkeit erwarten lassen.

Für die wirtschaftliche und gesellschaftliche Entwicklung der meisten Länder werden in den nächsten Jahrzehnten vor allem folgende Triebkräfte von ausschlaggebender Bedeutung sein:

- die moderne Informationstechnologie,

- die Globalisierung der Wirtschaft,

- die fortschreitende Europäisierung von Wirtschaft und Politik,

- der weltweite Bewußtseins- und Einstellungswandel,

- die ökologischen Grenzen und

- Bevölkerungswachstum, -wanderungen und -strukturwandel.

Gemeinsam werden sie, darin sind sich die meisten Beobachter und Analytiker der Entwicklung des industriellen Kapitalismus einig, zu weltweiten Verteilungskämpfen ungeahnten Ausmaßes führen, die nicht nur auf wirtschaftlicher, sondern auch auf kultureller Ebene ${ }^{8}$ ausgetragen werden. Sie werden sich in den ent-

\footnotetext{
8 Huntington, Samuel P.: The Clash of Civilizations. Deutsche Ausgabe. Europaverlag, München und Wien, 1996.
} 
wickelten Volkswirtschaften in sozialen Kämpfen niederschlagen, von denen wir schon heute erste Anzeichen verspüren.

Von den Triebkräften sind die ersten drei für unser Thema von direkter Bedeutung, worauf ich gleich detaillierter eingehen werde. Darüber dürfen aber die mehr indirekten Auswirkungen der anderen Triebkräfte nicht vergessen werden. Denken Sie beispielsweise daran, daß ökologische Probleme zunehmend auch unseren Konkurrenten in der Dritten Welt Wachstumsschranken auferlegen. Dies mag zwar die Kostenrelationen etwas zu unseren Gunsten mildern, stellt aber für diese Länder zweifellos eine herbe Beeinträchtigung ihrer Wachstumschancen dar. Oder denken Sie an die grundlegenden Probleme des Bewußtseins und Einstellungswandels. Alte gewachsene, stabile Kulturen werden innerhalb weniger Jahre von Grund auf umgepflügt, was - Stichwort Fundamentalismus - in vielen Teilen der Welt zu erheblichen Reaktionen führt. Und die Völker, die sich an die neuen Verhältnisse, etwa in Südostasien, angepaßt haben und die auf die wirtschaftliche Entwicklung, die dort angestoßen worden ist, vertrauen, müssen nun erleben, daß Reif auf ihre Blütenträume fällt. Auch dies gibt in den nächsten Jahrzehnten zu massiven internationalen Verteilungskämpfen Anlaß, denen wir notgedrungen, jedenfalls zum Teil, nachgeben werden müssen?.

Und denken Sie bitte schließlich auch daran, daß weltweit, mit Ausnahme Westeuropas, die Bevölkerung weiterhin erheblich wächst. Sie wächst vor allem in den Ländern mit niedriger wirtschaftlicher Leistungsfähigkeit und niedrigem wirtschaftlichen Potential. In den entwickelten Volkswirtschaften wächst sie wesentlich langsamer. Auch hiervon gehen erhebliche globale Rückwirkungen aus. Wir erleben in Deutschland die Auswirkungen solcher Entwicklungen durch die direkten Bevölkerungszuwanderungen. Ganz anders als vor 30 Jahren, wo sie erwünscht waren, um das Arbeitskräftepotential aufzufüllen, sind sie heute, von den Rentenfachleuten einmal abgesehen, durchaus unerwünscht, weil sie zu erheblichen sozialen Spannungen und wirtschaftlichen Problemen führen.

9 In schöner Offenheit formuliert das Sprachrohr der Londoner Hochfinanz, der "Economist", daß natürlich die "taxpayer" und natürlich die der entwickelten Volkswirtschaften die in Not geratenen Notenbanken der „kleinen Tiger“ inklusive Südkorea, aus ihrer Notlage auslösen müssen. Vergl. zum Beispiel „Reality hits Japan“ , „The Economist" vom 19. November 1997. 
Nun komme ich zu den direkten Wirkungen von Informationstechnologie und Globalisierung der Wirtschaft. Ihre weitreichenden Konsequenzen beginnen wir im Grunde erst in den letzten 5 bis maximal 15 Jahren zu begreifen. Basis dieser Entwicklungen ist der säkulare Trend der neuen Informationstechnologie. Darunter verstehe ich einmal die Entwicklung der Mikroelektronik, die vor etwa 50 Jahren mit der Erfindung des Transistors einsetzte und die in der jüngeren Zeit eine progressive Beschleunigung erfahren hat. Ich meine aber auch die Entwicklung der "Software", die daran anknüpft. Das sind nur zum geringeren Teil die neu entwickelten Strukturen, Algorithmen und Programme, die erst die Funktion der "Hardware" ermöglichen. Ich meine vor allem auch die daraus folgende Adaption der gesellschaftlichen Organisationsformen.

Eine erste wichtige Konsequenz dieser Entwicklung ist: sie steigert die Produktivität in allen Wirtschaftszweigen in einem historisch einmaligen Ausmaß. Es ist außerordentlich wichtig, daß die Produktivitätssteigerung so gut wie alle Bereiche erfaßt. Die technischen Fortschritte, die wir bisher in der Wirtschaftsgeschichte des industriellen Kapitalismus erlebt haben, haben zunächst immer nur die Produktivität spezieller Bereiche erhöht, was dann zu positiven "spill-overs" in andere Wirtschaftsbereiche geführt hat. Ein gutes Beispiel ist die Entwicklung der Eisenbahn. Sie hat die ganze Pferdefuhrwerks-Technologie ruiniert. Der Aufbau des Eisenbahnnetzes hat aber auch im Lauf der Zeit zur direkten Beschäftigung einer großen Zahl von Arbeitskräften im Eisenbahnwesen geführt und über die Stahlnachfrage die ganze Stahltechnologie eigentlich erst wirklich zum Blühen gebracht. Auch ihr Produkt, Transportleistungen, hat in anderen Wirtschaftszweigen zu erheblichen Wachstumssteigerungen und damit verbunden zu zusätzlicher Beschäftigung geführt.

Die Entwicklung der Mikroelektronik verläuft nach einem anderen Muster. Zwar hat auch sie erheblich zur direkten Beschäftigung einer immer noch steigenden Zahl von Arbeitskräften beigetragen. Ich habe eine Schätzung für die USA im Kopf, die in der Größenordnung von drei Millionen liegt. Für die spill-overs, die sie auslöst, gilt dies freilich nicht. Ihre Produkte erhöhen die Produktivität in allen Wirtschaftsbereichen dadurch, daß sie Arbeitskräfte ersetzen. Sie ermöglichen komplexe Steuerungs- und Regelungsprozesse und setzen dadurch in der klassischen industriellen Produktion auch höher qualifizierte Arbeitskräfte frei. Selbst in 
Dienstleistungsbereichen finden heute, von Friseuren und ähnlichen unmittelbar personenbezogenen Dienstleistungen einmal abgesehen, massive Produktivitätsfortschritte durch die Freisetzung von Arbeitskräften statt. Symptomatische Beispiele hierfür sind das "electronic ticket" der Fluggesellschaften und das "electronic banking" unserer Banken. Ironie der Geschichte: Mikroprozessoren werden sogar zum Entwurf komplexerer Mikroprozessoren eingesetzt und ersetzen damit einige der Programmierer, von denen sie ursprünglich entwickelt worden waren. Die Revolution frißt auch hier ihre eigenen Kinder.

Die neue Informationstechnologie beeinflußt aber nicht nur das Ausmaß benötigter menschlicher Arbeitskraft, sie schafft auch neue Arbeitsstrukturen: Direkt durch die Entwicklung neuer Berufsbilder, wie Programmierer, Systemanalytiker, Operateur etc., aus denen sich eine neue Elite bildet, und indirekt über die Veränderung der bestehenden Arbeitsorganisation.

Das erste einschlägige Stichwort hierfür lautet „neue Selbständigkeit", häufig auch abqualifizierend als "Scheinselbständigkeit" bezeichnet. Nur ist das so eine Frage. Ich kenne aus meiner früheren Universitätslaufbahn Assistenten, die ganz bewußt kein unselbständiges Arbeitsverhältnis mehr eingehen, sondern sich im Werkvertrag verdingen. Immer mehr Menschen wählen freiwillig die neue Selbständigkeit, unter anderem auch, um Sozialversicherungsbeiträge zu sparen und eine eigene Form der Altersvorsorge vorzunehmen. Es ist also keineswegs nur so, daß Unternehmen Mitarbeiter freisetzen oder auf dem Wege des Outsourcing in solche neuen "Arbeitsverträge" drängen, denen es an der sozialen Sicherheit des unbestimmten Arbeitsverhältnisses für unselbständige Arbeitnehmer gebricht. Natürlich gibt es das in großem Stil. Es gibt aber auch eine gesellschaftliche Nachfrage nach solchen Arbeitsmöglichkeiten. Angebot und Nachfrage werden dazu führen, daß ihre Bedeutung in der Zukunft weiter wächst.

Das zweite Stichwort lautet „Telearbeitsplatz". Vielleicht sollte man lieber sagen „virtuelle Arbeitsorganisation“. Diese Formulierung macht besser deutlich, worum es eigentlich geht. Der direkte räumliche Konnex verliert unter den technischen Möglichkeiten, die die neue Informationstechnologie bietet, an Bedeutung. Räumlich weit voneinander getrennt residierende Mitarbeiter können simultan interaktiv gemeinsam an der Lösung von Problemen 
arbeiten. Die Vernetzung macht es möglich. Das gilt nicht nur für die Finanzwelt oder die Welt des Programmierens, für die wir dies wissen und erwarten würden. Es gilt beispielsweise auch für die Konstruktionsbüros großer Automobilkonzerne, die rund um die Welt Spezialisten mit der Entwicklung neuer Modelle beschäftigen. Und es gilt für die in Telekonferenzen kommunizierenden Boards, Vorstände und Managementgremien, deren Mitglieder früher einmal in demselben Gebäude hausen mußten, um ihre Zusammenarbeit zu garantieren, die heute aber zu echten virtuellen Veranstaltungen geworden sind.

Das dritte Stichwort heißt "neue Lebensarbeitszeitkarriere". Die inzwischen klassische Arbeitskarriere begann damit, daß man mit 14 eine Lehre begann oder mit 18 ein Studium, an deren bzw. dessen Ende man in den Betrieb eintrat. In diesem verblieb man, bis man mit 65 pensioniert wurde. Das ging zum Teil über Generationen so. Es gab an der Ruhr Familien, bei denen schon der Urgroßvater „beim Hoesch" war, dann der Großvater, der Vater und nun auch der Sohn. Die Zeiten sind vorbei - und dies nicht nur, weil es "den" Hoesch so nicht mehr gibt. Das Muster „erst Ausbildung - dann produktive Arbeit mit intermittierendem Jahreserholungsurlaub - dann Ruhestand" wird für eine steigende Zahl von Menschen der Vergangenheit angehören.

In zunehmendem Maß werden die im globalen Wettbewerb stehenden Unternehmen Vorreiter bei den Bemühungen sein, das Modell der Beschaffung materieller Produktionsmittel "just in time" auf die Arbeitsleistungen zu übertragen, um Arbeitskosten zu senken und die Produktion flexibler an die in ständigem Wandel befindlichen Märkte anzupassen. Arbeitsleistungen werden von den Arbeitskräften nur noch in der Form definierter Zeitscheiben abgerufen werden. Eine solche Entwicklung würde auch damit korrespondieren, daß angesichts der Dynamik der technischen und der wirtschaftsorganisatorischen Entwicklung die Arbeitsqualitäten in mehr oder weniger regelmäßigen Abständen angepaßt werden müssen. Kürzere (aber immer noch mehrjährige) Perioden der Arbeit würden mit Perioden einer Fortbildung und/oder Umschulung abwechseln. Es ist abzusehen, daß das Rechtsinstitut des auf unbestimmte Zeit abgeschlossenen Arbeitsvertrags einmal als Charakteristikum der vergangenen Periode des kapitalistischen Hochindustrialismus im Abendland angesehen werden wird. 
Mit dem Rückgang der Bedeutung dieses Rechtsinstituts geraten aber alle anderen gesellschaftlichen Institute, die daran anknüpfen, in Bedrängnis. Es entsteht dann doch das Problem, daß alle diese intermittierend an wechselnden virtuellen Standorten Tätigen nicht mehr nach dem für die Steuerbehörden so billigen und bequemen Quellenabzugsverfahren besteuert werden können. Für die deutsche Sozialversicherung werden die Probleme noch viel gravierender sein, weil sie begrifflich direkt an diesem Arbeitsverhältnis ansetzt - anderenfalls wäre ein solches begriffliches Monstrum wie "Lohnersatzfunktion" gar nicht denkbar.

Aber auch die rein technischen Probleme werden sich vermehren. Wie kommt denn die Behörde oder die Sozialversicherung überhaupt an ihre Schäfchen ran? Würden wir zum Beispiel eine Sozialversicherungspflicht für diese Leute einführen? Was wird aus dem Territorialprinzip, wenn diese Werktätigen neuer Art ihre Arbeitsleistungen von überall, wo sie sich gerade befinden, in das Inter- oder ein sonstiges Net einschleusen?

Mit all diesen Problemen muß man rechnen. Und ich sage eins dazu, wir stehen erst am Vorabend dieser Entwicklungen. So wäre es ganz naiv anzunehmen, daß wir etwa durch ein Gesetz gegen die 610/520-Mark-Regelung die ganze Entwicklung stoppen könnten. Das wäre allenfalls ein Schlag ins Wasser; neue Dauerarbeitsplätze im traditionellen Sinn würden dadurch nicht geschaffen. Es führte vielleicht zu einer kurzen Zwischenepisode, während der man möglicherweise auf der zweiten Stelle hinter dem Komma etwas für die Beitragssätze 'rausholt. Aber ganz sicher hat dieses Vorhaben nichts Ernsthaftes damit zu tun, neue Quellen für die Finanzierung der gesetzlichen Krankenversicherung zu erschließen, und noch weniger ist es geeignet, sich gegen den Strom der Zeit zu stemmen.

Der zweite säkulare Trend ist die Globalisierung. Die Globalisierung verschiebt die politischen Machtverhältnisse und die wirtschaftlichen Machtverhältnisse säkular. Die nationalen Organisationen verlieren an Macht, während die regionalen Organisationen und globalen Organisationen an Macht gewinnen. Ich sage bewußt "Organisationen", weil es politische wie wirtschaftliche wie gesellschaftliche Einrichtungen sein können. Auslösend für diese Verschiebungen ist der Machtgewinn der globalen Organisationen gegenüber den nationalen. Dieser beruht darauf, daß sie sich jederzeit in ihrem globalen Netzwerk dem Zugriff eines einzelnen 
Nationalstaats entziehen ${ }^{10}$ können. Sie zwingen damit die Nationalstaaten in einen Wettbewerb untereinander, sofern die Nationalstaaten auf die Präsenz der globalen Organisationen angewiesen sind - und das sind sie, weil sie deren Wertschöpfung als Basis für Beschäftigung und Steuereinnahmen brauchen. Dies alles läuft unter dem Schlagwort des Wettbewerbs der immobilen um die mobilen Faktoren. Eine Ausnahme hiervon können sich wegen ihrer schieren Größe allenfalls die Vereinigten Staaten und - in zunehmendem Maß - auch China leisten.

Die regionalen Organisationen gewinnen deshalb, weil die nationalen an Macht verlieren. Symptomatisch hierfür ist die weltweit zu beobachtende Regionalisierung, die sich mit so konträren Erscheinungen wie Euregiones und Subsidiarität einerseits und mit "ETA" oder „Legio Nord" andererseits verbindet. Diese Entwicklung ist sicherlich zu begrüßen, soweit es sich darum handelt, rein regionale Phänomene in regionaler Selbstbestimmung und Autonomie zu regeln. Nach aller menschlichen Erfahrung darf man aber davon ausgehen, daß sich der Ehrgeiz regionaler Autoritäten ${ }^{11}$ nicht hierauf beschränken lassen wird.

Hauptproblem in unserem Zusammenhang ist, daß die Globalisierung es den Nationalstaaten unmöglich macht, eine effektive nationale Arbeitsmarktpolitik zu verfolgen. Globale Unternehmen sind in der Lage, die Produktionsstätten frei zu wählen und damit Arbeitsplätze dort zu schaffen, wo sie es für sie am günstigsten erachten. In einer globalisierten Welt ist jeder Arbeitsplatz eine „verlängerte Werkbank". Da gibt es den Stammbetrieb nicht mehr, auf den sich ein Unternehmen durch Abstoßen der verlängerten Werkbänke zurückzieht, wenn es Anpassungsprobleme hat. Das war das strukturpolitische Problem der 60er Jahre. Heute ist das alles vorbei. Heute sind alle Arbeitsplätze verlängerte Werkbänke, sofern sie nicht einen rein örtlichen oder rein regionalen Bedarf befriedigen. Weder die Putzfrau, die mal eben putzt, noch der Friseur, der Ihre Haare schneidet, noch der Masseur oder die Pediküre werden je vom Trend zur Globalisierung erfaßt werden.

10 Um deutlich zu machen, daß dieses Phänomen keineswegs nur auf die viel gescholtenen „Multis" beschränkt ist, bezeichne ich es gerne ohne jede moralische Wertung als das "Schreinemakers-Syndrom".

11 Ebenfalls ohne jede moralische Wertung könnte man dies als das "Stoiber-Syndrom" bezeichnen. 
Bereits vor der eigentlichen Globalisierung hat die Internationalisierung der Wirtschaft durch internationalen Handel eine weitgehende Verkettung der Arbeitsplätze bewirkt. Die Auswirkungen dieser Entwicklung auf die Löhne'12 wurden erstmals von den skandinavischen Nationalökonomen Heckscher und Ohlin beschrieben. Bildlich gesprochen, bekommen wir durch den Weltwirtschaftshandel einen Weltarbeitsmarkt. Die Vorstellung von vor 20 Jahren, daß wir in Deutschland im Rahmen einer internationalen Arbeitsteilung die Blaupausen machen und die anderen die Dreckarbeit, liegt leider völlig daneben. Programmiert wird nicht nur bei uns, sondern hauptsächlich in Indien, so daß der Flop bei der Programmierung, den die GKV kürzlich hat hinnehmen müssen, wahrscheinlich nicht hier in Deutschland produziert worden ist, auch wenn das Unternehmen, das den Auftrag übernommen hatte, noch einen deutschen Namen trägt. Wie bereits gesagt, werden neue Kraftfahrzeuge in einem Computernetz weltweit konstruiert. Das sind ganz andere Strukturen als die, die aus der Vorstellung von einer weitgehenden internationalen Arbeitsteilung resultieren.

Also, wir haben heute einen sich homogenisierenden Weltarbeitsmarkt. Und zwar nicht nur für die Spitzenqualitäten, wie im Management oder bei der darstellenden Kunst, sondern für alle Arbeitsqualitäten. Ein deutscher Arbeiter bei Volkswagen konkurriert mit seinen Kollegen in Japan und Korea nicht direkt, sondern weil ihre Produkte in der Vorstellung ihrer Käufer Substitute sind. Je ähnlicher diese Produkte werden, je intensiver der Wettbewerb zwischen innen wird, je mehr sich, technisch gesprochen, die Produktmärkte homogenisieren, um so stärker wird auch der Druck auf eine Angleichung der Preise nicht nur der Produkte, sondern auch der Löhne der Arbeiter, die sie produzieren.

Internationalisierung und Globalisierung der Weltwirtschaft führen dazu, daß in allen entwickelten Volkswirtschaften der Druck auf die Einkommen aller Arbeitsqualitäten immer stärker werden wird. Diese Entwicklung ist unausweichlich. Auch die Gewerkschaften können dem nicht gegensteuern. Sie werden an Macht und Bedeutung verlieren. Wir leben im Augenblick in einer historischen Phase, in der nach nahezu 150 Jahren gegenläufiger Entwicklung, wenn ich es einmal in den Begriffen von vor 150 Jahren

12 Allgemeiner : die Preise aller Produktionsfaktoren. 
ausdrücke, Kapital wieder mächtiger und Arbeit wieder ohnmächtiger wird.

Das hat unmittelbare Rückwirkungen auf eine Wirtschaft wie die unsere, die zu den höchst entwickelten Volkswirtschaften mit dem höchsten Wohlstand und dem am besten ausgebauten System der sozialen Sicherheit und der sozialen Gerechtigkeit durch Umverteilung gehört. Wir laufen in eine Periode sich verschärfender Verteilungskämpfe. Wir müssen mit jahrelanger hoher Arbeitslosigkeit rechnen. Der Druck auf Einkommen und Sozialsysteme wird anhalten, ja, sich eher erhöhen.

Bei jährlichen Steigerungsraten der Arbeitsproduktivität um 3-4, $5 \%$ wären rein rechnerisch reale Wachstumsraten der Gesamtnachfrage in gleicher Höhe erforderlich, um die Beschäftigung zu halten. Sowohl die Inlandsnachfrage als auch der Exportüberschuß müßten jährlich real mit derselben Rate wachsen. Ich frage Sie, was sollte da produziert werden, und wer sollte diese Produkte abnehmen, um sie zu investieren oder zu konsumieren? Die Inlandsmärkte dürften solche Wachstumsraten kaum bringen. Wir haben in vielen Bereichen weitgehend gesättigte Märkte. Selbst der Bierkonsum stagniert sogar in Bayern. Und wo wären im Inland die Nachfragebereiche, in welchen wegen dauerhaft hoher Wachstumsraten des Absatzes in Kapazitätserweiterung investiert werden würde?

Es kommt noch eines hinzu. Vor der Tür stehen 1,2 Milliarden Chinesen, die zu einer stürmischen industriellen Entwicklung gestartet sind. Sie haben schon heute sehr zum Schaden der jungen Tiger in den internationalen Wettbewerb eingriffen. Es ist abzusehen, daß sie eines Tages auch uns erhebliche Probleme bereiten werden.

Dies gilt um so mehr, als wir zur Anpassung an den globalen Wettbewerb im Gegensatz zu den USA ein Modell exerzieren, das zwar verteilungsgerecht, aber beschäftigungsschädlich ist. Unsere globalen Wettbewerber produzieren u. a. wegen ihrer niedrigeren Löhne billiger. Unsere Unternehmen reagieren darauf durch Rationalisierung. Das Ausmaß dieser Rationalisierung hängt von der Höhe der Reallöhne ab. Je höher die Reallöhne sind, um so größer ist der Anreiz zu arbeitsplatzsparenden Rationalisierungsinvestitionen. Diese führen zu zusätzlichen Arbeitslosen. Dennoch fordern unsere Gewerkschaften Lohnerhöhungen 
im Ausmaß des Produktiviätsfortschritts, weil sie immer noch an die alte Formel von der produktivitätsorientierten Lohnpolitik glauben. Früher verhieß dies eine Sicherung des Wachstums „auf des Messers Schneide". Heute verschärft eine solche Politik das Beschäftigungsproblem, weil solche Lohnerhöhungen die angestrebte Verbesserung der Reallohnposition gegenüber den Drittländern wieder zunichte machen.

Früher glaubte man, diese Rückwirkung vernachlässigen zu können. Es war die allgemeine Überzeugung der auf der neoklassischen Wirtschaftstheorie fußenden Wirtschaftspolitik, daß Entwicklungsländer angesichts des Arbeitsüberschusses und der Kapitalknappheit immer zu arbeitsintensiveren Produktionstechniken griffen als wir. Noch in den 60er Jahren hat die Bundesrepublik Entwicklungshilfe $u$. a. dadurch betrieben, daß sie von der Industrie ausrangierte Maschinen gekauft und an Entwicklungsländer verschenkt hat. Man hat geglaubt, diese zögen entsprechend ihres relativen Reichtums an Arbeit und ihrer relativen Knappheit an Kapital die arbeitsintensiven Techniken vor. Dies hat sich als völliger Irrtum herausgestellt. Aus metaökonomischen Erwägungen heraus investieren Schwellenländer, ja sogar startups wie China, in die modernste für sie erreichbare Technologie. Sie investieren praktisch genauso modern, kapitalintensiv, produktiv wie wir, aber sie haben weiterhin ihre niedrigeren Löhne. Doch selbst, wenn sie ihre Löhne nach der Produktivitätsformel anpassen würden, lägen sie immer noch niedriger als bei uns.

Im Ergebnis halten wir unsere internationale Wettbewerbsfähigkeit, wie man unschwer an der Entwicklung unserer Handelsbilanz erkennt. Das geschieht aber zum Preis einer steigenden Zahl von Arbeitslosen. Daß es auch anders geht, belegen die USA. Diagramm 2 zeigt die Entwicklung der Arbeitslosenraten in den USA, Deutschland und Frankreich. Die dünn gestrichelte Linie stellt die USA dar. Man sieht, daß die Arbeitslosenquote in den USA bei allen Vorbehalten, die man gegenüber deren Methode der Messung haben muß, im wesentlichen konstant verläuft, während ihre Entwicklung in Deutschland und Frankreich praktisch seit den 70er Jahren einen steigenden Trend aufweist. 


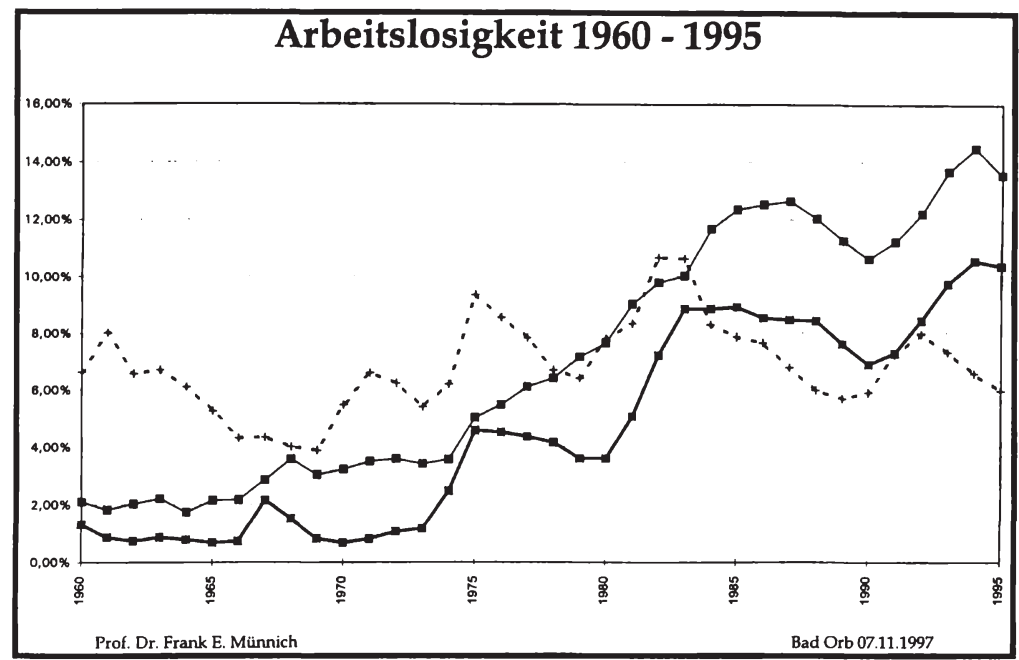

Natürlich zahlen die USA hierfür einen Preis, denn nichts im Leben ist umsonst. Der Preis liegt in der Entwicklung der Einkommensverteilung ${ }^{13}$. Die ist schlicht katastrophal. Sie ist katastrophal, weil der Erfolg bei der Bekämpfung der Arbeitslosigkeit, das „amerikanische Beschäftigungswunder", wie es neoliberale Kreise bei uns gerne nennen, von den Ärmsten der Armen bezahlt worden ist. Die beiden untersten Fünftel der Einkommensverteilung der Vollzeit arbeitenden männlichen Arbeitskräfte, von denen der Hauptbeitrag zum Familieneinkommen erwartet wird, haben von 1973 bis 1992 einen realen Einkommensverlust von mehr als $20 \%$ hinnehmen müssen. Nur das oberste Fünftel der Einkommensbezieher konnte sein Einkommen steigern, und das um $10 \%$. Die USA zeigen damit in den beiden letzten Jahrzehnten eine Spreizung der Einkommensverteilung, wie sie sonst nur von Feudalstaaten bekannt ist. Bei uns konnte trotz sinkender Lohnquote die reale Einkommenssituation auch der Bezieher niedriger Einkommen in etwa aufrechterhalten werden.

Bei den geschilderten Trends handelt es sich um langfristige, säkulare Prozesse, die durch keine nationale Politik beseitigt werden können. Nationale Politik kann lediglich ihre negativen Kon-

13 Die Zahlen finden sich in Lester Thurow : Die Zukunft des Kapitalismus. Metropolitan Verlag, Düsseldorf und München 1996. S.41. 
sequenzen abmildern und die Gesellschaft auf den unvermeidlichen Strukturwandel vorbereiten. Die Schlußfolgerung aus alledem lautet daher, daß auf mittlere und längere Frist an der Beschäftigungsbezogenheit unserer sozialen Sicherungssysteme und damit an der Lohnbezogenheit ihrer Finanzierung nicht festgehalten werden kann. Tiefgreifende Strukturänderungen müssen angepackt werden.

Von den durchschnittlichen Tendenzen kann die reale Entwicklung natürlich von Periode zu Periode durchaus abweichen. Es sind diese kurzfristigen Aberrationen, auf die die Politik in der Regel reagiert. Man kann aber den langfristigen Entwicklungen nicht mit kurzfristig konzipierten Ad-hoc-Maßnahmen begegnen. Man kann sie nur durch strukturelle Maßnahmen, durch grundlegende Rahmenbedingungen in die gewünschten Bahnen lenken. Die Politik der Bundesregierung, welcher Couleur auch immer, wird um solche Maßnahmen nicht herumkommen. Auch die Beteiligten werden ihre Einstellungen und Strategien anpassen müssen.

Eine Kette von aufeinanderfolgenden Notfallmaßnahmen, wie wir sie seit zwei Jahrzehnten unter der Überschrift Gesundheitspolitik erleben, läßt zwar das System von Mal zu Mal gerade eben überleben. Offensichtlich ist die Schmerzgrenze für eine durchgreifende Reform aus einem Guß noch nicht erreicht. Daher ist derzeit wohl auch keine andere Politik möglich und sollte daher auch nicht angestrebt werden. Das entbindet freilich nicht von der Notwendigkeit, eine solche Politik intellektuell vorzubereiten - frei nach dem Motto: denke in der Zeit, dann weißt Du in der Not, was zu tun ist - und sie in den von Fall zu Fall aktuell erforderlichen kleinen Schritten vorbereitend anzulegen.

Ich will einige für Finanzierung relevante strategische Felder anreißen, für die solche strategischen Konzepte entwickelt werden müßten und die Gegenstand der folgenden Beratungen sein könnten.

\section{Beschäftigten- oder Volksversicherung}

Als das "Gesetz, betreffend die Krankenversicherung der Arbeiter" 1883 geschaffen wurde, war die Industriearbeiterschaft im sozialen Spektrum eine Minorität. Es gab mehr Menschen, die als Hausgehilfen oder in der Landwirtschaft beschäftigt waren. Erst im Laufe der Zeit wurde der Kreis der versicherten Personen langsam ausgeweitet. Auch die Reichsversicherungsordnung von 
1911 brachte keineswegs den allgemeinen sozialen Krankenversicherungsschutz. Erst in der Zeit der Bundesrepublik wurde durch Urteil und Gesetz der Anteil der sozial Krankenversicherten auf den heutigen Satz von etwa 92 Prozent hochgeschraubt.

Die Ausdehnung der Versicherungspflicht folgte allzeit vor allem politischen oder fiskalischen Motiven; auch die Justiz hat daran aus vermeintlicher Rechtssystematik mitgewirkt. Es ist daher dringend an der Zeit, den Kreis der Versicherungspflichtigen nach dem Kriterium der Schutzbedürftigkeit systematisch neu zu bestimmen, zumal, wie ausgeführt, das Kriterium der (unselbständigen) Beschäftigung seine Sinnhaftigkeit in Zukunft völlig verliert. Es gibt gute, sachliche Gründe sowohl für eine Reduktion der Versicherungspflicht auf einen sehr enggefaßten Kreis sehr bedürftiger Menschen als auch für die Ausdehnung auf alle zu einer echten Volksversicherung. Als ersten Schritt auf dem Wege zu einer solchen Volksversicherung kann man eine Anhebung der Versicherungspflichtgrenze interpretieren.

\section{Finanzierung über Beiträge oder Steuern}

Traditionell wird die deutsche gesetzliche Krankenversicherung durch Beiträge finanziert. Aus der Sicht der Sozialpolitiker und der Sozialversicherungsträger hat das den Vorteil, daß das Aufkommen, sieht man einmal von den mehr oder weniger raffiniert kaschierten Schleichwegen der Verschiebebahnhöfe ab, den Begehrlichkeiten all der anderen an den öffentlichen Töpfen Nagenden entzogen ist. Genau dies läßt sich aber auch kritisch sehen. Man kann sich durchaus auf den Standpunkt stellen, daß es ein Unding sei, daß ein quantitativ derartig bedeutender Zweig der öffentlichen Finanzgebarung den allgemeinen politischen Zielinterdependenzen so vollständig entzogen bleibt.

Weniger ein prinzipielles Problem, sondern eines der konkreten Ausgestaltung sehe ich in der ausgesprochenen Regressivität unseres Beitragssystems. Man könnte ihm mühelos dadurch Rechnung tragen, daß man vom Lineartarif abrückt. Konsequenterweise müßte man dann allerdings auch die Beitragsbemessungsgrenze und die Versicherungspflichtgrenze aufheben.

Auf dem Weg zur idealtypischen Alternative, der Steuerfinanzierung, gibt es eine Fülle von Zwischenstufen und Detailvorschlägen, denn nirgends fühlt sich die menschliche Kreativität so herausgefordert wie beim Erfinden oder Vermeiden von Steuern. 
Systematisch am saubersten im Hinblick auf das bestehende System wäre wohl noch die Ausdehnung der Beitragsbemessung auf weitere Einkommensarten. Dies gibt es bereits bei den freiwillig versicherten Rentnern. Ich teile die Zuversicht nicht, daß die technischen Probleme einer Ausdehnung auf alle Versicherten negligeabel seien. Die gemeinsame Selbstverwaltung hat noch nie versagt, wenn es darum ging, aus vermuteten technischen Problemen echte zu machen.

Es gibt dabei auch offene systematische Fragen, wie etwa die nach den einzubeziehenden Einkommensarten. Warum nicht gleich alle? Auch dies wäre noch lange keine Steuerfinanzierung im eigentlichen Sinne. Es würden immer noch von den Sozialversicherungsträgern Beiträge erhoben. Nur deren Bemessung würde sich nach dem gesamten steuerbaren Einkommen des Versicherten berechnen. Brutto, versteht sich; dann aber nur bis zu einem Höchstbetrag.

Diskutiert werden auch Staatszuschüsse. Hierfür stehen zwei Muster zur Verfügung: einmal die deutsche Rentenversicherung, die Zuschüsse für die sogenannten versicherungsfremden Leistungen erhält, und zum anderen die Schweiz, die in einem recht diskussionswürdigen, im wesentlichen marktorientierten System Zuschüsse zur Umsetzung des Solidarausgleichs verteilt.

Last, but not least könnte die Finanzierung der gesetzlichen Krankenversicherung direkt aus dem öffentlichen Budget erfolgen. Dann allerdings hätten wir ein ganz anderes System. Es würde vielleicht mehr dem entsprechen, was Bismarck ursprünglich im Auge hatte, als das, das seinen Namen trägt.

\section{Hochrisiko- oder Vollversicherung}

Kollektive Vollversicherung aller ist angesichts des heutigen Leistungspotentials der Medizin und ihrer Zulieferindustrien einerseits und der strukturellen Grenze für Umverteilung andererseits nicht mehr möglich. Damit wird der Leistungsumfang zum Problem systematischer Analyse und Entscheidung. Es geht dabei, dies muß immer wieder betont werden, nicht darum, was das Medizinsystem leistet, sondern ausschließlich darum, welche dieser Leistungen aus Zwangsbeiträgen kollektiv finanziert werden. Alle anderen Leistungen muß der Patient privat versichern oder aus eigener Tasche zahlen. 
Die Idee der sozialen Solidarität fordert, daß alle "großen" Risiken von der Solidargemeinschaft zu übernehmen sind. Subsidiarität rechnet dem einzelnen die Dinge zu, die er aus eigenem tragen kann, und delegiert die anderen an die umfassende Gemeinschaft. Beide Definitionen sind nicht scharf genug. Allein die generelle Existenz eines ökonomischen Problems belegt, daß es für jeden von uns Dinge gibt, die er nicht aus eigenem zu tragen vermag. Es bedarf also in jedem Fall eines politischen Konsenses darüber, was dem einzelnen zu tragen zugemutet wird.

Ich liebe es, die individuelle Zumutbarkeit anhand des Anteils des eigenen Einkommens zu definieren, den man für die in Anspruch zu nehmenden Leistungen aufwenden muß. Dieses Konzept entspricht der Behandlung außergewöhnlicher Belastungen bei der Einkommenssteuer. Sein großer Vorteil ist seine Einfachheit, sein Nachteil in unserem Zusammenhang die Anfälligkeit gegenüber einkommenssteigernden Strategien der medizinischen und perimedizinischen Leistungserbringer.

Wie die Existenz von Hochrisikoversicherungen beweist, können die Probleme aber pragmatisch gelöst werden. Man kann sie finanzwirtschaftlich durch ein gestaffeltes System von Zuzahlungen angehen. Die realwirtschaftliche Alternative liegt darin, den Leistungskatalog zu beschränken. Das von mir entwickelte Vierbzw. Dreistufenmodell für Zuzahlungen bei Arzneimitteln ist der Versuch, beide Aspekte miteinander zu verbinden.

\section{Sozialausgleich}

Traditionell ist die deutsche gesetzliche Krankenversicherung ein ehrwürdiges Beispiel für die Funktionstüchtigkeit der alten Marxschen Vision: „Jedem nach seinen Bedürfnissen, jeder nach seiner Fähigkeit". Die GKV beinhaltet einen umfassenden Sozialausgleich, der allerdings einige allgemein bekannte Unerträglichkeiten aufweist, etwa die Diskriminierung der berufstätigen Ehefrauen und die Privilegierung der Ehefrauen, die ohne Belastung durch Erziehung von Kindern das traurige Dasein eines "tortenfressenden Pelztieres" fristen. Ein verteilungspolitisches Hauptärgernis ließe sich durch Aufhebung der Beitragsbemessungsgrenze beseitigen, ein anderes durch die Einbeziehung weiterer Einkommensarten in die Beitragsbasis und die Abschaffung des linearen Tarifs, ein drittes schließlich durch die Abschaffung der 
kostenfreien Mitversicherung von Familienangehörigen, sofern sie keiner zumutbaren Tätigkeit nachgehen.

Die systematische Lösung aller Verteilungsfragen liegt auch hier wieder in einer grundlegenden Systemumkehr. Die richtige Stelle für eine konsistente Lösung aller Verteilungsprobleme ist das Budget, über das alle Transfers fließen sollten. Natürlich müßten dann die Träger der gesetzlichen Krankenversicherung entsprechende Zahlungen aus den allgemeinen Budgets erhalten. Hierfür könnte auch ein eigener Parafiskus geschaffen werden, der zwischen dem Budget und den Versicherern stünde. Die bereits zitierten National Economic Research Associates stellen sich vor, daß die Versicherten ihre einkommensabhängigen Beiträge direkt - also ohne den "Umweg“ über das öffentliche Budget - in einen solchen Fonds einzahlen. Meine eigene Lieblingsvorstellung ist die Übertragung des trotz aller Mängel im Prinzip bewährten Wohngeldmodells auf die GKV. Der besondere allokative Charme aller dieser Ansätze liegt darin, daß die Versicherer mit risikoäquivalenten Beiträgen bedient werden könnten. Spätestens dann stünde allerdings auch die künstliche Trennung zwischen GKV und PKV zur Disposition.

\section{Familien- oder Individualansprüche}

Ein weiteres charakteristisches Strukturelement unserer ganzen sozialen Sicherung ist nur aus der Sozialstruktur des vergangenen Jahrhunderts verständlich, die Familienbezogenheit der Ansprüche. In der Rentenversicherung ist sie Gegenstand verfassungsrechtlicher Klärung gewesen. Auch das Scheidungsrecht hat inzwischen davon Kenntnis genommen. In der gesetzlichen Krankenversicherung dauert der Kuddelmuddel bedauerlicherweise aber weiter an. Erstaunlicherweise hat sich sogar die private Krankenversicherung, in der man wahlweise Familienverträge oder Individualverträge haben kann, gegenüber dieser Sozialromantik als anfällig erwiesen. Ich denke, daß die hochgradige Mobilität unserer Gesellschaft gerade auch in Fragen von Ehe und Familie nahelegt, in Zukunft auf familienbezogene Ansprüche völlig zu verzichten und durchgehend zum Individualprinzip überzugehen. 


\section{Lösung der Beiträge von den Lohnkosten}

Ebenfalls von der Herkunft der GKV her ist die paritätische Finanzierung der Beiträge zu verstehen. Man kann darüber streiten, ob diese Finanzierung tatsächlich „paritätisch“ ist. Ich stimme der Auffassung $z u$, daß die Arbeitgeberanteile in Wahrheit Lohnbestandteile sind. Sie werden von den Arbeitgebern bei Einstellung eines Mitarbeiters als solche kalkuliert und sind, wie viele Finanzwissenschaftler überzeugend argumentieren, längst überwälzt. Lediglich der Anteil an einer Erhöhung der Beiträge trifft zunächst die Arbeitgeber voll. Hierdurch gerät die Politik in ein Dilemma. Zur Sicherung der medizinischen Versorgung für alle und zur Sicherung der mehr als 3 Millionen Arbeitsplätze im Gesundheitswesen - wäre es dringend erwünscht, zusätzliche Mittel in das Gesundheitswesen zu kanalisieren. Diese würden aber über die Arbeitgeberanteile direkt die Lohnkosten erhöhen und so die internationale Attraktivität und Wettbewerbsfähigkeit des Standorts Deutschland beeinträchtigen.

In der Politik wird derzeit als Einstieg in den Ausstieg aus diesem Dilemma die Festschreibung des Arbeitgeberanteils diskutiert. Aus Illusion, Vorurteil und mangelnder Phantasie ist aber selbst dieser kleine Schritt offensichtlich nicht konsensfähig. Die richtige systematische Lösung ginge viel weiter: Es wäre die einmalige steuerneutrale Ausbezahlung der Arbeitgeberanteile. Von da an wäre die GKV eine reine Versichertenversicherung. Ein im Grunde systemfremdes Element wäre eliminiert. Allerdings auch die paritätische Besetzung der Aufsichtsgremien der Krankenkassen. Die schon jetzt bestehende Ausnahme bei den Ersatzkassen beweist, daß eine solche Systemänderung ohne Schaden möglich wäre. Weil soziale Organisationen aber mindestens ebenso vom Selbsterhaltungstrieb beherrscht werden wie biologische Wesen, stößt eine solche Reform auf den erbitterten Widerstand der Arbeitgeber und der Gewerkschaften, gegen den die Politiker auch dann nichts zu unternehmen wagten, wenn sie von dem Konzept überzeugt wären. So bleibt denn die wirklich grundlegende Frage, wie unsere soziale Krankenversicherung angelegt sein sollte, ob, wie bislang, als "Selbstverwaltung" oder als Staatsverwaltung oder als sozialverpflichteter privater Wirtschaftsbereich, weiterhin aus jeder, auch dieser Diskussion außen vor.

Ich schließe. Ich sage voraus, was immer geschieht, die Finanzierungsproblematik bleibt. Soziale Probleme werden nie gelöst, sie 
werden nur von anderen abgelöst. Ich sage auch, daß die Finanzierungsproblematik unabhängig von der Basislösung bestehen bleibt. Dies gilt auch für eine ausschließliche Finanzierung über Steuern, weil die Faktoren, auf die ich hingewiesen habe, für das allgemeine Steueraufkommen unter Umständen noch schlimmere Konsequenzen haben als für Beiträge, die sich am Lohn orientieren. Den immobilen Unselbständigen kann die Behörde unbedenklich filzen, das Kapital aber nicht. Das verschwindet notfalls irgendwo hinter den Antillen. Bislang ist noch fast jeder Versuch, auch die Zinsen angemessen zu besteuern, schmählich gescheitert. Die Ausgrenzung von Leistungen ist also unumgänglich.

Ich denke, daß viele politische Probleme wegen der Globalisierung nur noch auf europäischer Ebene lösbar sind. Dazu gehören auch die Fragen der Besteuerung. Deswegen halte ich es auch für so wichtig, daß der Euro kommt und daß wir zu einer europäischen Wirtschaftspolitik gelangen. Dann glaube ich, daß wir auf Dauer mit Ad-hoc-Maßnahmen nicht auskommen werden. Irgendwann stehen wir vor dem großen Offenbarungseid. Dann helfen nur noch schmerzliche Revolutionen. Bei diesem grundlegenden Strukturwandel geht nichts ohne Ausgrenzungen, ohne Eingrenzung des Leistungskatalogs. Dann geht auch nichts ohne Staatszuschüsse. Und mit der in sich zerstrittenen Selbstverwaltung ist es dann auch vorbei. Irgendwann, sage ich voraus, landen wir bei einer Volksversicherung aus Steuermitteln. Das wäre dann ein später Triumph für Biedenkopf - wenn er inn je erleben sollte. 


\section{Strukturverträge und Modellvorhaben \\ - neue Tätigkeitsfelder für die GKV}

Gerd Glaeske

Um die Entwicklung zu den GKV-Neuordnungsgesetzen auch in bezug auf die Paragraphen $63 \mathrm{ff}$. und 73 a SGB V verstehen und bewerten zu können, in denen den Krankenkassen bzw. den Verbänden die Möglichkeit eingeräumt wird, über Modellvorhaben oder Strukturverträge nach einer Optimierung der medizinischen Versorgung suchen zu können, ist eine kurze Analyse der derzeitigen Situation der gesetzlichen Krankenversicherung (GKV) in Deutschland notwendig.

Seit einigen Jahren werden in einer breiten Öffentlichkeit verstärkt die Kosten unseres Gesundheitswesens und die Bezahlbarkeit der medizinischen Versorgung in der Zukunft diskutiert. Dabei gelten die Argumente des demographischen Wandels, der für das Jahr 2029 einen Anstieg von derzeit rund $16 \%$ auf rund $24 \%$ der Menschen über 65 Jahre prognostiziert, und der medizinische Fortschritt als besonders kostentreibende Faktoren in der $\mathrm{Zu}$ kunft. Während der Ausgabenanstieg für den medizinischen Fortschritt in der Tat schwer kalkulierbar ist, wird der Generationenwandel vielfach kritiklos und auch unberechtigt als Grund für dramatische Kostensteigerungen in der Zukunft dargestellt. Schätzungen gehen davon aus, daß im Jahre 2030 die Behandlungskosten im Rahmen der gesetzlichen Krankenversicherung insgesamt um etwa $10 \%$ gegenüber den Ausgaben in Höhe von 228,8 Mrd. DM im Jahr 1995 ansteigen werden (STATISTICS).

Dennoch kann nicht verwundern, daß verstärkt nach Strategien gesucht wird, um die Kosten für die Ausgaben im Gesundheitssystem zu senken, obschon sich in der Zwischenzeit auch bei Politikern die Überzeugung breit gemacht hat, daß wir weder eine Kostenexplosion in den vergangenen Jahren $\mathrm{zu}$ verzeichnen hatten - schließlich ist der Anteil der Ausgaben für Gesundheit, gemessen am Bruttoinlandsprodukt, zwischen 1985 und 1995 in der Bundesrepublik gerade einmal von $8,7 \%$ auf $9,6 \%$ gestiegen - im Vergleich dazu in den USA von $10,7 \%$ auf $14,5 \%$ und in Frankreich von $8,5 \%$ auf $9,9 \%-$, sondern daß vor allem Einnahmenprobleme für die schwierige Finanzierungssituation der Sozialversicherungssysteme geltend gemacht werden müssen. 
Solange die Finanzierung der Sozialversicherungssysteme wie der GKV alleine auf dem unsicher werdenden Faktor Arbeit lastet und immer weniger Menschen ein regelmäßiges Arbeitseinkommen erwirtschaften, da die Anzahl der Arbeitslosen ansteigt (Ende des Jahres 1997 betrug sie immerhin knapp 4,6 Millionen), werden die Belastungen der verbleibenden Grundlohnsumme und damit die Beitragssätze für die Sozialversicherungssysteme wie gesetzliche Krankenversicherung und Rentenversicherung weiter steigen. Diese Entwicklung bedeutet aber letztlich steigende Lohnnebenkosten, die die "Arbeit" in der Bundesrepublik teurer machen als in Ländern mit niedrigeren Löhnen und geringerer Arbeitslosigkeit. Wenn dann noch aus politischen Gründen zur Entlastung der Arbeitslosenversicherung die Beitragsbemessungsgrenze für die Beiträge in der GKV von $100 \%$ auf $80 \%$ abgesenkt werden, so bedeutet dies mit einem Federstrich die Verringerung der Einnahmen für die GKV in Höhe von 5-6 Mrd. DM, immerhin ein Betrag, der rund 0,3 Beitragssatzpunkten entspricht und fast zwangsläufig zu Beitragssatzanhebungen zumindest in dieser Höhe führen muß (1 Beitragssatzpunkt entspricht derzeit ca. 18 Mrd. DM).

\section{Die Leistungsanforderungen in der GKV}

Für die gesetzliche Krankenversicherung gelten Rahmenbedingungen, die durch das 5 . Sozialgesetzbuch (SGB V) definiert sind. So heißt es im $\S 12$ des SGB V: „Die Leistungen müssen ausreichend, zweckmäßig und wirtschaftlich sein; sie dürfen das $\mathrm{Ma}$ des Notwendigen nicht überschreiten. Leistungen, die nicht notwendig oder unwirtschaftlich sind, können Versicherte nicht beanspruchen, dürfen die Leistungserbringer nicht bewirken und die Krankenkassen nicht bewilligen." Im § 2 wird für die Leistungen in der GKV gefordert: „Qualität und Wirksamkeit der Leistungen haben dem allgemein anerkannten Stand der medizinischen Erkenntnisse zu entsprechen und den medizinischen Fortschritt zu berücksichtigen." Und im $\S 70$ heißt es, quasi zusammenfassend, unter der Überschrift Qualität, Humanität und Wirtschaftlichkeit: "Die Krankenkassen und die Leistungserbringer haben eine bedarfsgerechte und gleichmäßige, dem allgemein anerkannten Stand der medizinischen Kenntnisse entsprechende Versorgung der Versicherten zu gewährleisten. Die Versorgung der Versicherten muß ausreichend und zweckmäßig sein, darf das $M a ß$ des Notwendigen nicht überschreiten und muß wirtschaftlich erbracht werden. Die Krankenkassen und die Lei- 
stungserbringer haben durch geeignete Maßnahmen auf eine humane Krankenbehandlung ihrer Versicherten hinzuwirken."

Im Bereich der ambulanten Versorgung durch Ärztinnen und Ärzte existieren Gebührenordnungen, die den Wert der ärztlichen und sonstigen medizinischen Leistungen festlegt. Damit sollen die Kosten für die medizinische Versorgung kalkulierbar werden; es kommt zu einer festen Gebühr, wie sie auch bei anderen Dienstleistungen üblich ist und die von den jeweiligen Krankenkassen an die Ärztinnen oder Ärzte für die ambulante medizinische Versorgung der Versicherten im Rahmen einer Gesamtvergütung gezahlt wird. Damit ist der Markt nun vollkommen aufgehoben, frühe Gesundheitsökonomen sprechen sogar von einem 'Nicht-Markt' (z. B. Liefmann-Keil): Einer fragt die Leistung nach, ein zweiter erbringt sie, ein dritter zahlt sie.

\section{Der Einfluß von Gebührensystemen auf die medizinische Versorgung}

Vergütungssysteme prägen aber die Struktur eines medizinischen Versorgungssystems (Glaeske). Systeme, die auf der Abrechnung von Einzelleistungen aufgebaut sind, indem sie alle denkbaren einzelnen Leistungen finanziell bewerten (fee-for-service), fördern das Angebot und die Anwendung solcher Leistungen, die wegen ihrer hohen Honorierung attraktiv für die Leistungserbringer sind. Es reduziert umgekehrt Leistungen ohne attraktives Honorar. Wenn z. B. der Faktor "Zeit" nicht adäquat honoriert ist, wird man auf Leistungen, die vor allem zeitintensiv erbracht werden, vergeblich hoffen.

Die unerwünschte, medizinisch vielfach nicht notwendige Mengenausweitung ist ebenso Folge der Einzelleistungsvergütung wie die Tatsache, daß Patienten, also kranke Versicherte, als ökonomisches Potential im derzeitig vorherrschenden Vergütungssystem gelten. Ein Versicherter muß krank sein, damit das ärztliche Versorgungssystem an inm verdient. Dies läßt auch die Vermutung aufkommen, daß manche Krankheiten durch das medizinische Versorgungssystem nicht nur definiert, sondern auch „produziert" werden: Die Festsetzung von Grenzwerten in der Diskussion um die Hyperlipidämien sind hierfür ein typisches Beispiel. Der angeblich pathologische Wert $200 \mathrm{mg} / \mathrm{dl}$ hat über die Hälfte aller Menschen von einem Tag zum anderen als krank definiert und damit zu behandlungsbedürftigen Patienten erklärt. Ein 
älterer Mensch, der zu unterschiedlichen Ärzten geht, wird immer wieder aufs neue als krank definiert werden müssen, damit jeder ein entsprechendes Behandlungshonorar abrechnen kann. Bei jedem Arztkontakt wird statistisch gesehen ein Arzneimittel verordnet - geradezu als Bestätigung der pathologischen Befunde. Eine solche Behandlungsintensität ist aber auch Konsequenz des zunehmenden ökonomischen Drucks und des Konkurrenzverhaltens der Ärztinnen und Ärzte untereinander: Jeder will an einem Patienten verdienen, die Mengenexpansion ärztlicher Leistungen ist auch hiermit zu erklären. Daß aber viele dieser angeblich pathologischen Befunde auf altersbedingte Veränderungen gegenüber einem 30-40jährigen zurückgehen, wird zu wenig berücksichtigt - Abweichungen vom "Normalen", damit aber auch Alterserscheinungen, werden als Krankheit definiert - sicherlich einer der Gründe für die oftmals irrationalen Arzneimittelmengen, die älteren Menschen verordnet werden. Und daß viele niedergelassene Ärzte bei ambulant durchführbaren Behandlungs- oder Untersuchungsprozeduren lieber ins (teure) Krankenhaus einweisen als zu einem spezialisierten Kollegen zu überweisen, hat auch damit zu tun, daß die Patienten aus dem Krankenhaus in die eigene Praxis zurückkommen, nach einer Überweisung zu einem ebenfalls ambulant tätigen Kollegen ist dies weniger sicher („vom Feindflug nicht zurückgekehrt!"). Durch eine solche Praxis kommt es aber zu einer unnötigen Zunahme der Fallzahlen und Leistungsintensität im Krankenhaus; Kooperationsdefizite führen letztlich zu Versorgungsdefiziten. Im Mittelpunkt dieses Versorgungssystems steht die kurative Medizin, ein sicherlich vielfach erfolgreicher, aber häufig auch unnötig oder fragwürdig angewandter Reparaturbetrieb. Die Mengenausweitung im System der Einzelleistungsvergütung fördert zudem Einbußen in der Qualität, da Ärzte vielfach apparategestützte Leistungen erbringen, ohne ausreichend Erfahrungen mit diesen Diagnostik- und Therapieverfahren gesammelt zu haben. Gut dotierte apparategestützte diagnostische Verfahren fördern überdies Doppeluntersuchungen oder die nicht indizierte Ausweitung oder sogar Fragwürdigkeit der Anwendung solcher Verfahren. Hierfür ist die Knochendichtemessung sicherlich ein aktuelles Beispiel, die nach wie vor als Screeningmethode zur Entdeckung von osteoporosekranken Frauen eingesetzt wird, hierfür aber nach Meinung von Fachleuten ungeeignet ist (Lange et al.). Dies hat aber auch mit der Kundenorientierung niedergelassener Ärzte in einem System der Einzelleistungsvergütung zu tun: Im patientenorientierten Wettbewerb der Ärzte untereinander steht neben dem Ruf, ein guter Arzt 
zu sein, auch der Wettbewerb der Praxisausstattung, die allein über die Verfügbarkeit der möglichst neuesten apparativen Angebote hohe Leistungsbereitschaft und Qualität signalisiert, ohne dies in jedem Fall nachgewiesen zu haben. High-Tech-Praxen müssen sich aber amortisieren, die Einzelleistungsvergütung bietet die entsprechende Voraussetzung. Die Nutzung der Verfahren bei möglichst vielen Patienten, ob medizinisch notwendig oder nicht, schafft die Abzahlungsquote - das Hamsterrad beginnt sich zu drehen! Schon relativ banale Erkrankungen ziehen die Anwendung der gesamten Palette diagnostischer und therapeutischer Verfahren nach sich: Maximalversorgung hat aber nur wenig mit einer optimalen Versorgung zu tun.

Die pauschalierten Honorierungsformen haben andere Auswirkungen: Der ökonomische Anreiz für die Ärzte besteht gerade nicht in der Mengenausweitung, sondern in der Minimierung. Ärzte werden in einem solchen System nach der Anzahl der Patienten honoriert („capitation“), die in einem vereinbarten Zeitraum (Quartal oder Jahr) die Praxis aufsuchen. Daher werden die Ärzte versuchen, jeden Behandlungsfall möglichst kostensparend abzuwickeln, um einen möglichst hohen Anteil der Pauschale ohne entsprechende Arbeitsgegenleistung in Anspruch nehmen zu können. Sie werden auch möglicherweise versuchen, nur leicht kranke Patienten in ihr Klientel aufzunehmen, die wenig Kosten verursachen. Dennoch wird der Arzt eine Balance zwischen Leistungsangebot und Kostenminimierung finden müssen, weil bei fortgesetzter Untätigkeit enttäuschte Patienten die Praxis zum nächstmöglichen Zeitpunkt wechseln werden.

Die mögliche Auswirkung einer ärztlichen Untätigkeit trägt dem pauschal honorierten Versorgungssystem häufig den Vorwurf ein, übrigens besonders häufig von Ärzten in Einzelleistungsvergütungssystemen, potentiell unterversorgend zu wirken und Qualitätseinbußen der Versorgung quasi billigend in Kauf zu nehmen. Abgesehen davon, daß dieses Argument einen erstaunlichen Blick auf ärztliche Ethik zuläßt, gelten vor allem zwei andere Gründe, die gegen eine systematische Qualitätsreduktion sprechen:

- Der Wettbewerbsdruck um die Patienten. (...) Dieser Wettbewerbsdruck ist daher Voraussetzung für die Effizienz der Honorierung nach Kopfpauschalen. 
- Das Haftungsrecht, das Abweichen vom professionellen Standard untersagt und nachhaltige Sanktionen gegen eine Kostenminimierung auf Kosten der Gesundheit der Patienten enthält.

Empirische Vergleiche zeigen im übrigen, daß sich die Versorgungsqualität unter einem Pauschalvergütungssystem nicht verschlechtert. So konnte bei einem Vergleich der Blutdruckbehandlung in Großbritannien und Deutschland festgestellt werden, daß die Patientenversorgung unter der geringeren Aktivität der pauschaliert honorierten britischen Ärzte nicht gelitten hat (Donner-Banzhoff). Dies mag auch daran liegen, daß in solchen Systemen akzeptierte Therapieempfehlungen oder Leitlinien den Behandlungsprozeß des Patienten begleiten, die als Standard berücksichtigt werden. Eine ungerechtfertigte Abweichung vom Standard könnte aber möglicherweise haftungsrechtliche Konsequenzen für den behandelnden Arzt haben. Pauschalierte Systeme bieten damit einen finanziellen Anreiz für die Gesunderhaltung eines in der Praxis eingeschriebenen Versicherten. Nicht Kuration, sondern Prävention oder Hinweise für einen gesunden Lebensstil werden daher eine stärkere Betonung in der Versorgung bekommen.

Daher ist aus einem Vergütungssystem auch die "Philosophie" einer medizinischen Versorgung abzulesen: Die hohe Bewertung technischer bzw. einzelorganbezogener Leistungen perpetuiert ein Patientenmodell, das maschinenähnlich auf einzelne Schwachstellen diagnostiziert und repariert werden kann, es fördert eine "manipulative Medizin", eine hohe Bewertung des Faktors "Zeit", der Raum läßt für Gespräche, Beratungen, technisch nicht gestützte Anamnesen, reflektiert den Patienten als "ganzen“ Menschen, als einen Patienten, bei dem auch psychosomatische Ursachen zur Krankheit führen können, es fördert eine „interpretierende Medizin“. Mit der Förderung der jeweiligen Philosophie geht auch eine unterschiedliche Förderung von Arzttypen einher: Das erste System fördert den Spezialisten und damit den Gebietsarzt, das zweite den Generalisten und damit den Allgemeinarzt. Eine Balance zwischen beiden Systemen käme sicherlich einer angemessenen und patientenorientierten medizinischen Versorgung am ehesten entgegen. Daher gibt es Vorstellungen über ein rationales Vergütungssystem, das möglichst folgende Aspekte berücksichtigen sollte: 
- keine Anreize zur Mengenausweitung (z. B. bei diagnostischen/ technischen Leistungen)

- Anreize für zuwendungsorientierte Leistungen

- Anreize zur Sicherung der notwendigen Fachkunde

- Unterbindung überflüssiger Doppeluntersuchungen

- funktionsgerechte Arbeitsteilung in der medizinischen Versorgung zwischen ambulantem und stationärem Sektor und innerhalb dieser Sektoren

- einfache Prüfmöglichkeiten sowie

- Flexibilität und Anpassungsfähigkeit an veränderte Umstände.

Honorierungssysteme haben also Auswirkungen auf das Versorgungssystem - es gibt nichts Strukturierenderes als eine Gebührenordnung. Dies gilt übrigens nicht nur für die ambulante Versorgung, sondern auch für den stationären Bereich. So können z. B. Fallpauschalen Strategien nach sich ziehen, Patientinnen und Patienten nach Operationen frühzeitig in eine kostengünstige Reha-Einrichtung zu verlegen, mit denen ein Krankenhaus einen Vertrag zur weiteren Betreuung abgeschlossen hat, um Kapazitätserhöhungen im eigenen Haus und damit häufigere Abrechnungen der Fallpauschalen realisieren zu können. Es kommt auch, wie einzelne Beispiele zeigen, zu doppelten Abrechnungen der Fallpauschalen, wenn Patienten kurzfristig, z. B. für einen Tag, entlassen und am folgenden Tag wieder aufgenommen werden. Auch dies führt zu einer Erhöhung von Fallzahlen mit z. T. denselben Patienten. Und schließlich können Fallpauschalen Risikoselektionen nach sich ziehen, weil bei Patienten mit schwerwiegenden Begleiterkrankungen das Problem einer Unterdeckung durch eine Fallpauschale bestehen könnte. Solche Risikoselektionen wirken sich damit direkt unter dem Aspekt der Ökonomie gegen die Patienten aus. All dies sind Auswirkungen, die schon theoretisch aus Gebührensystemen ableitbar sind. Daß diese Überlegungen auch praktische Auswirkungen haben, zeigen viele Beispiele.

\section{Überversorgung als Rationalisierungspotentiale, Unterversorgung als Herausforderung}

Es kann gar kein Zweifel daran bestehen, daß in unserem medizinischen Versorgungssystem Wirtschaftlichkeitsreserven bestehen, die durch falsch gesetzte Anreize in den geltenden Gebührensystemen zu erklären sind. Insgesamt werden die Ausgaben für unnötige oder fragwürdige medizinische Leistungen auf rund 
$10 \%$ aller Ausgaben geschätzt, also auf rund $25 \mathrm{Mrd}$. DM - so auch Gesundheitsminister Seehofer in einem Interview in der „Welt am Sonntag" im Februar 1996. Diese finanziellen Fehlallokationen kommen z. B. durch die mangelnde Verzahnung des ambulanten und stationären Bereichs zustande: Doppeluntersuchungen und nicht notwendige diagnostische oder auch therapeutische Interventionen sind an der Tagesordnung. So schätzt der ehemalige Präsident der Deutschen Röntgengesellschaft, Professor Gerhardt aus München, daß etwa $30 \%$ aller Röntgenaufnahmen überflüssig sind, zum großen Teil wegen der sowohl ambulant als auch stationär durchgeführten bildgebenden Diagnostik (Gerhardt). Er hat weitere interessensbedingte Mengenausweitungen festgestellt: So röntgen Teilradiologen, also Z. B. Orthopäden oder Urologen mit eigener Röntgenausstattung in der Praxis, viermal häufiger als Ärztinnen oder Ärzte aus den gleichen Fachgebieten ohne Röntgenausstattung. Aber auch bei anderen Beispielen für die bildgebende Diagnostik können Probleme nicht übersehen werden, die alleine in der Mengenentwicklung liegen. Im Bereich des klassischen Röntgens beobachten wir trotz der Nutzung anderer bildgebender Verfahren einen Anstieg von $11 \%$ zwischen 1989 und 1992. Die Anzahl der Computertomogramme für Kopf- und Wirbelsäulendarstellungen hat sich im selben Zeitraum von 350.000 auf 700.000 verdoppelt, die der Kernspinresonanzuntersuchungen an Kopf und Wirbelsäule sogar von 50.000 auf 300.000 versechsfacht. Waren diese diagnostischen Maßnahmen immer medizinisch notwendig und begründbar oder wurden sie nicht auch als Konsequenz eines falschen ökonomischen Anreizsystems durch die Gebührenordnungen induziert, die immer das gleiche Honorar für eine bildgebende Diagnostik ausweisen, obwohl sich das jeweilige Untersuchungsgerät nach einer Reihe von Jahren längst amortisiert hat? Und ist diese lukrative Erwartung nicht auch Grund für die Überkapazitäten in der fachärztlichen Anwendung von Großgeräten?

Derzeit besonders diskutiert werden die unterschiedlichen Strategien bei der Versorgung von Herzinfarkt- oder Koronarpatienten (ÄrZTE-Zeitung). Bypass-Operationen, Angiographien oder die Ballondilatation bei Gefäßverschlüssen sind in diesen Fällen die häufigsten Verfahren in der Kardiologie. Auffällig sind allerdings die unterschiedlichen Häufigkeiten, mit denen diese Verfahren in verschiedenen Ländern durchgeführt werden. Professor Salim Yusuf aus Hamilton in Kanada hat nun die Ergebnisse einer solchen Vergleichsstudie (Organisation to Assess Strategies for 
Ischemic Syndroms / OASIS) in den Ländern Brasilien, USA, Kanada, Australien, Ungarn und Polen vorgelegt, in der es um Patienten mit akuter Koronarerkrankung ging. Insgesamt bezogen die Untersuchungen rund 8.000 Patienten mit akutem Koronarsyndrom ein. Die höchsten Nutzungsraten dieser Verfahren liegen in den USA und in Brasilien. In beiden Ländern war der Anteil der Patienten, bei denen innerhalb der ersten Woche in der Klinik eine Angiographie oder Ballondilatation durchgeführt wurde, jeweils etwa dreimal höher als in den übrigen Ländern (Angiographie $58,4 \%$ versus $20,2 \%$, PTCA $15,9 \%$ versus $4,9 \%$ ), die Rate der Bypass-Operationen lag sogar um den Faktor sieben höher $(11,5 \%$ versus $1,6 \%)$. Die Ergebnisse waren dann allerdings frappierend: Trotz der unterschiedlichen Behandlungsstrategien bewegten sich die Raten der aufgetretenen Todesfälle oder akuten Herzinfarkte in den unterschiedlichen Ländergruppen auf nahezu gleichem Niveau. Nach sechs Monaten fiel die Rate an Todesfällen und Infarkten sogar leicht zuungunsten der beiden Länder mit der intensiveren Strategie aus (11,3\% versus $10,7 \%)$.

Etwas anderes wurde in der Studie offensichtlich: Die Krankenhäuser, die über alle technischen Möglichkeiten verfügten, zeigten auch eher die Bereitschaft, diese Techniken zu nutzen, eine Strategie, die zumindest nach den erhobenen Daten für die Patienten nicht unbedingt von Vorteil war. So wurden 4.674 Patienten einer aggressiven invasiven Therapie unterzogen, 3.200 einer eher konservativen Therapie.

Eine weitere Studie (Veteran Administration Non-Q-Wave-Infarction-Strategies in Hospital/VANQUISH) kam zu ähnlichen Ergebnissen, auch hier kam es in der aggressiver behandelten Gruppe zu einer höheren Zahl von Todesfällen. Auch die Dauer der Behandlung war deutlich länger als bei der konservativ behandelten Gruppe.

Professor William E. Boden aus Syracuse im Staat New York, der Leiter dieser Studie, kam zu dem Schluß, daß die von vielen Ärzten geradezu reflexartig durchgeführte Katheterisierung bestimmter Infarktpatienten als ungünstig entlarvt worden sei. Und er empfiehlt dringend, zunächst eine konservative Therapie zu wählen, bis sich der Zustand des Herzinfarktpatienten stabilisiert hat, bevor im Einzelfall über weitergehende Maßnahmen entschieden wird. Fazit: Es sind nicht die maximalen Möglichkeiten der Medizin, die dem Patienten nützen, es sollten vielmehr die für 
ihn optimalen in der jeweiligen Situation angewendet werden. Dies gilt vor allem dann, wenn sich unnötig aggressive Strategien sogar negativ auswirken. Ähnliche Ergebnisse liegen auch für die Bundesrepublik Deutschland vor. So berichtete Prof. von Leitner über erste Ergebnisse eines Projekts der leitenden Krankenhauskardiologen zur Anwendung von Herzkathetern bzw. einer Ballondilatation von Herzkranzgefäßen, daß von zirka 25.000 Eingriffen, die pro Jahr durchgeführt werden, etwa 6.000 als „ungerechtfertigt" klassifiziert werden müssen. Dabei bedeutet "ungerechtfertigt", daß die Patienten besser mit einem anderen Verfahren behandelt werden sollten, z. B. mit einer medikamentösen Therapie oder mit einer Bypass-Operation (FRANKFURTER RUNDSCHAU).

Insgesamt können gerade die Kardiologie und die Kardiochirurgie als Beispiel dafür gelten, daß möglicherweise in den vergangenen Jahren die Indikationsstellung für einzelne Behandlungsmaßnahmen unkritisch ausgeweitet wurde und es zu einer erheblichen, wenn nicht problematischen Mengenausweitung kam:

Die Behandlungskapazitäten sind erheblich ausgebaut worden: So standen Anfang 1996356 Linksherzkatheter-Meßplätze zur Verfügung, 76 Herzzentren waren in Betrieb. Zwischen 1990 und 1995 war eine Verdoppelung der Häufigkeit von Herzoperationen in den alten Bundesländern feststellbar - von rund 35.000 auf 70.000 Operationen. Verdoppelt hat sich im gleichen Zeitraum auch die Untersuchungsanzahl der LinksherzkatheterUntersuchungen - von rund 200.000 auf 386.000 . Parallel zu dieser Entwicklung gab es, wiederum im gleichen Zeitraum, auch eine Verdoppelung der Bypass-Operationen - von 25.000 auf 51.000. Die Häufigkeit der PTCA (Ballonkatheterdilatation) hatte sich sogar verdreifacht - von 35.000 auf 95.000 (Bruckenberger). Bislang gibt es keine ausreichend sicheren Analysen dazu, ob die Anstiege nicht teilweise auf eine angebotsinduzierte Nachfrage zurückgehen, ob also unangebrachte Indikationsausweitungen hierfür verantwortlich sind. Wenn dies aber der Fall ist, könnte eine Steuerung des Leistungsgeschehens, verbunden mit einer schärferen Indikationsstellung, solche Fehlentwicklungen auf die Basis der unter medizinischen Aspekten notwendigen Versorgung zurückführen.

Therapiestrategien müssen also immer wieder überdacht werden - weniger ist auch in der Kardiologie manchmal mehr! 
Auch Krankenhauseinweisungen können unnötig sein: So wurde eine Studie des medizinischen Dienstes der Krankenkassen veröffentlicht, die $22 \%$ aller Krankenhausfälle als Fehlbelegung bewertet, weil die zur Einweisung führenden Behandlungsanlässe wirtschaftlicher im ambulanten Bereich hätten versorgt werden können.

Rationalisierungsreserven liegen auch im Bereich der Arzneimittelversorgung. So werden Jahr für Jahr Arzneimittel verordnet, die in ihrer therapeutischen Wirksamkeit und in ihrem Nutzen für die Patienten als umstritten gelten oder deren Zusammensetzung als obsolet betrachtet werden muß. Die Ausgaben für diese Arzneimittelgruppen betragen immerhin 7,1 Mrd. DM, $22 \%$ aller Ausgaben für Arzneimittel im Rahmen der GKV. Viele dieser Mittel müssen durch nützliche und ausreichend wirksame Mittel ersetzt werden. Dennoch verbleibt ein geschätztes Einsparpotential von 2-3 Mrd. DM, weil Mittel wie z. B. topische Venenmittel, Leberund Gallenwegstherapeutika oder viele Enzym-, Mineral- und Vitaminpräparate sowie pflanzliche Immunstimulantien völlig eingespart werden können, ohne die Qualität der Arzneimittelversorgung zu verschlechtern (Schwabe/Paffrath).

Neben der Überversorgung sind aber auch Felder einer Unterversorgung bekannt. So hat z. B. D. Hölzel 1996 darauf hingewiesen (Bericht in der Süddeutschen Zeitung vom 11.01.1996), daß nach der Einführung des Wirkstoffes Cisplatin im Jahr 1979 die Heilungschancen von Hodentumoren für einen Teil der Patienten von 45 auf 90 Prozent verdoppelt wurden. Trotz der eindeutigen Resultate dauerte es dann etwa acht Jahre, bis alle Ärzte das neue Medikament als "Standard" akzeptierten. Diese verspätete Akzeptanz, so hat Hölzel berechnet, hat etwa 1.500 Patienten das Leben gekostet. Unterversorgung ist auch bei der Schmerztherapie onkologischer Patientinnen und Patienten zu beklagen. Wie wäre es sonst zu erklären, daß der durchschnittliche Morphinverbrauch in Dänemark, berechnet nach Tagesdosierungen pro Kopf der Einwohner, trotz ähnlicher Daten der Prävalenz von onkologischen Erkrankungen wie in der Bundesrepublik, um 10mal höher liegt (Wimmer)? Es scheint eine verbreitete Zurückhaltung davor zu bestehen, Schmerzpatienten individuell angemessen auch mit stark wirksamen Schmerzmitteln zu behandeln. Unterversorgung, legitimiert mit dem irrationalen Argument einer möglichen Abhängigkeitsentwicklung. 
Unterversorgung auch z. B. im Bereich der Versorgung von Diabetikerinnen und Diabetikern: Nach wie vor werden nur bei rund der Hälfte der Patienten regelmäßig Untersuchungen des $\mathrm{HbA1c}$ Wertes durchgeführt, der als wichtigster Parameter für eine normoglykämische Einstellung gilt, ebenso selten Untersuchungen des Augenhintergrundes, des Fußpulses und der Nierenfunktion. Dabei sind diese diagnostischen Hinweise erforderlich, um den schwerwiegenden Spätfolgen wie Blindheit, diabetischer Angiopathie und Neuropathie mit der Spätfolge der Amputation und diabetischer Nephropathie mit der Spätfolge der Dialysepflichtigkeit vorzubeugen. Rund 28.000 Amputationen pro Jahr, 9.000 Erblindungen und mindestens $1.500-2.000$ neue Dialysefälle pro Jahr gehen zurück auf nicht ausreichend behandelte und betreute Diabetespatienten.

Die medizinische Versorgung muß daher rationaler werden, Überversorgung ist unnötig und belastet die Patienten, ohne den Krankheitsverlauf abzukürzen, Unterversorgung verzögert die mögliche Wiederherstellung der Gesundheit oder führt die Patienten in vermeidbare Risiken. Beides ist in höchstem Maß unethisch! Vor allem die Überversorgungstendenzen in unserem Gesundheitssystem, bedingt durch die weiter oben diskutierten falsch gesetzten Anreize in dem geltenden Gebührensystem, schaffen erhebliche Rationalisierungsreserven, die zugunsten der finanziellen Entlastung des Versorgungssystems genutzt werden müssen. Die Nutzung dieser Rationalisierungsreserven ist keineswegs mit einer verstärkten Kommerzialisierung des Versorgungssystems zu erreichen. Das Gegenteil ist der Fall: Die Kommerzialisierung der Patientenversorgung, wiederum Folge falsch gesetzter Anreize im Gebührensystem, muß beendet werden und Platz für Strukturen einer optimalen Versorgung machen. Die derzeitig aufgrund von Evaluationsstudien zu erkennende Maximalversorgung ist vielmehr Ausdruck eines kommerzialisierten Systems, das in individueller Weise vor allem von den Leistungsanbietern (aus)genutzt wird. Dieser Mißbrauch des Systems ist aber nicht nur auf Ärztinnen und Ärzte bzw. Institutionen im Versorgungssystem begrenzt, auch Patientinnen und Patienten tragen ihre Erwartungen und Ansprüche an dieses System heran, damit ihre Vorstellungen von Behandlungsintensität befriedigt werden. Insofern werden Ärzte und Patienten häufig zu „Komplizen“ in einem System, in dem allerdings das Machtgefälle eindeutig zuungunsten der Patienten verschoben ist und sie vielfach nur auf bereitgestellte Angebote reagieren. 


\section{Der „Lichtblick“ der NOGs: Modellvorhaben und Strukturverträge}

Um die Rationalität, die Qualität und die Wirtschaftlichkeit des Gesundheitssystems zu erhöhen, sind daher dringend Organisationsformen zu schaffen, die die Leistungsstrukturen, die Honorierungssysteme und die Behandlungsabläufe innovativ im Sinne einer wirtschaftlichen und indikationsgerechten Patientenversorgung gestalten. Diese Möglichkeit hätte mit den GKVNeuordnungsgesetzen bestanden, natürlich mit einer notwendigerweise stärkeren Regulierung der Anbieter im System, also der Ärztinnen und Ärzte, der Apotheken, der Krankenhäuser und aller anderen Lieferanten. Dieser Weg wurde aber nicht gewählt, einfacher durchsetzbar war offensichtlich die stärkere Belastung von Patienten im System, um die Beitragshöhe der gesetzlichen Krankenkassen auf einer akzeptablen Höhe zu halten und die Lohnnebenkosten nicht noch mehr als schon bislang zu „strapazieren“. Damit wurde letztlich der Weg gewählt, den Status quo aller bekannten Unwirtschaftlichkeiten und Unkoordiniertheiten zugunsten der Anbieter und der nicht erkrankten Versicherten im System beizubehalten und die finanzielle Entlastung der gesetzlichen Krankenversicherung über eine drastisch steigende Selbstbeteiligung von Kranken zu realisieren. Alleine im Arzneimittelbereich steigen die Zuzahlungen von 3,8 Mrd. DM ab dem Beginn des Jahres 1997 um weitere 3 Mrd. jährlich auf rund 6,8 Mrd. DM und damit auf etwa $20 \%$ der Gesamtausgaben in der GKV-Arzneimittelversorgung. Insgesamt wurden die Zuzahlungen so weit angehoben, daß knapp $12 \%$ aller Ausgaben - also rund $25 \mathrm{Mrd}$. DM - in der gesetzlichen Krankenversicherung nun von den Patientinnen und Patienten gezahlt werden - zusätzlich zu den bereits zwölfmal im Jahr gezahlten Beiträgen und ohne den hälftigen Arbeitgeberzuschuß (§§ 31, 32 und 33 SGB V). Diese "Philosophie" zieht sich insgesamt durch die NOGs: Sie beschreibt eine "Abkassierungsstrategie" bei den Patienten, die nicht nur durch ihre Krankheit, sondern auch durch erhöhte Zuzahlungen bestraft werden.

Neben dieser für eine solidarisch finanzierte gesetzliche Krankenversicherung wenig zukunftsfähigen Strategie, Patientinnen und Patienten an den Unwirtschaftlichkeiten eines Versorgungssystems zu beteiligen, um die Finanzierung auch zukünftig zu gewährleisten, gibt es einen kleinen Lichtblick in den NOGs: Die $\S \S 63 \mathrm{ff}$. und 73 a erlauben es, den Krankenkassen bzw. ihren 
Verbänden, neue Modelle für die Versorgung, für die Honorierung und für die Struktur der medizinischen Behandlung zu erproben. Dieses „Angebot“ des Gesetzgebers wollen viele Kassen und ihre Verbände in Zukunft für die Optimierung der Qualität und Wirtschaftlichkeit nutzen. Die Suche nach sinnvollen Alternativen zur derzeitigen Versorgung ist im übrigen Resultat des Wettbewerbsgedankens im Rahmen der solidarischen Krankenversicherung, der unter dem Aspekt von Wahlfreiheit und Risikostrukturausgleich dazu beitragen soll, den Kassen eine stärkere Beeinflussung des Kosten- und Versorgungsmanagements zu ermöglichen und damit eine wirksame Mittlerrolle zwischen Versicherten und Anbietern einnehmen zu können. Die nun gesetzlich eingeräumte Wahlfreiheit der Versicherten, die eine Wanderung zwischen den einzelnen gesetzlichen Krankenkassen, z. B. unabhängig von der beruflichen Eingruppierung als Arbeiter oder Angestellter, ermöglicht, muß in den einzelnen Krankenkassen auch Überlegungen fördern, welche Aspekte einen Versicherten bewegen, in einer Kasse zu bleiben oder ein Versichertenverhältnis in einer anderen Kasse zu beginnen. Hier spielen ohne Zweifel Beitragssatz, Serviceleistungen und Image eine bestimmende Rolle, sicherlich aber auch und immer mehr das Angebot medizinischer Beratungskompetenz und der Art der Versorgung im Ernstfall einer Erkrankung - wenn die Medizin für den einzelnen immer weniger "durchschaubar" wird, sollten sich Krankenkassen als Begleiter und Organisator einer wirksamen und patientenzugewandten Versorgung stärker als bislang profilieren. Sie müssen also Angebote machen, die für Kunden attraktiv sind. Sie müssen z. B. darstellen, welche besonderen Vorzüge es hat, bei ihnen versichert zu sein - gar nicht so einfach in einem System, in dem nicht über Leistungen Wettbewerb betrieben werden kann und soll, denn schließlich ist der Leistungsrahmen gesetzlich festgeschrieben, für alle gesetzlichen Krankenkassen gleich. Die Frage des (Kunden) Versicherten, welchen besonderen Vorteil es hat, in einer bestimmten Krankenkasse versichert zu sein, muß daher andere Antworten finden als den Hinweis auf Leistungen, die andere Kassen nicht bieten. Denkbar sind in diesem Zusammenhang andere Organisationsformen von Versorgung, die einem Versicherten mehr Sicherheit und Reibungslosigkeit einer Behandlung signalisieren als dies bei anderen Kassen der Fall ist: So sind z. B. Angebote einer integrierten Diabetesversorgung denkbar, die für den kranken Versicherten sowohl die Behandlung bei den Ärzten als auch die Versorgung mit Hilfsmitteln wie dem Blutzuckermeßgerät samt Teststreifen und Schulungsmaßnahmen in qualifizier- 
ten Schulungszentren organisieren. Ähnlich auch Angebote einer Hilfestellung für Schlaganfallpatienten, mit denen nicht nur ein möglichst rascher Übergang von der Akutversorgung in die Rehabilitation - entweder stationär oder ambulant - organisiert wird, sondern auch für das häusliche Umfeld notwendige Veränderungen in Gang gesetzt werden: Türschwellen oder Treppenstufen könnten schließlich unüberwindbare Hindernisse für einen Schlaganfallpatienten sein, der erst langsam wieder seine Motorik für ein sicheres Gehen zurückgewinnen muß. Die Wahlfreiheit der Versicherten setzt daher in den Krankenkassen ein sehr viel stärkeres Bewußtsein für Programme und Strategien in Gang, die für Versicherte attraktiv und gleichzeitig sinnvoll sind.

Andererseits verhindert der Risikostrukturausgleich (RSA), diese Angebote ohne Blick auf den Beitragssatz zu planen. Der RSA gleicht nämlich nur die Einnahmenseite der unterschiedlichen Kassen bis zum Durchschnitt der GKV aus, die Unterschiede in den Grundlohnsummen der Kassenmitglieder führen zwischen den einzelnen Kassen zu Geldtransfers: Liegt der Grundlohn aller Mitglieder, ergänzt um die Faktoren Alter, Geschlecht und Anzahl der Mitversicherten, unter dem Schnitt der GKV, wird die Kasse zur Empfängerkasse, liegt sie über dem Schnitt, wird sie zur Zahlerkasse. Nach dieser Methodik werden die Beitragseinnahmen einer Kasse, nicht aber die Ausgaben ausgeglichen. Wenn nun also eine Kasse meint, sich über besondere Attraktionen bei den Leistungen für Versicherte interessant machen zu können, dies sich aber auf einen ohnehin schon relativ hohen Beitragssatz auswirken würde, ist die Attraktion für bereits Versicherte oder potentielle Interessenten wahrscheinlich rasch dahin, weil die Höhe des Beitragssatzes eben auch ein wichtiges Wahlkriterium für eine Kasse darstellt. Die bisherigen Vorteile der Ersatzkassen eines durchschnittlich höheren Grundlohns gegenüber den AOKen werden also abgebaut, Vorteile für die einzelnen Kassen liegen zukünftig nur noch in einer unterdurchschnittlichen Ausgabenstruktur. Mit der mit dem RSA und der Wahlfreiheit betriebenen Auflösung des traditionellen Mitgliedschaftsrechts schwindet daher die Basis für den fast automatischen Mitgliederzufluß bei den Ersatzkassen. So ist die Tatsache, daß die BARMER in BadenWürttemberg ebenso wie im gesamten Bundesgebiet einen Beitragssatz von $13,9 \%$ erhebt, die AOK dort aber nur $13,0 \%$ fordert; unter Wettbewerbsbedingungen ausgesprochen folgenreich, da sowohl Versicherte wie aber auch Arbeitgeber durch eine AOK-Absicherung spürbare finanzielle Vorteile haben. Die jahre- 
lange Identität als Angestelltenkrankenkasse mit einem günstigen Beitragssatz aufgrund eines hohen durchschnittlichen Grundlohns trägt nicht mehr, da ein Teil des Beitragsaufkommens (ca. $15 \%$ ) RSA-relevant an Empfängerkassen überwiesen werden muß und nicht mehr für die Leistungen der eigenen Mitglieder zur Verfügung steht. Daß hierdurch aber Beitragssatzverwerfungen auftreten, ist mehr als plausibel. Wenn die BARMER daher in BadenWürttemberg eine höhere Attraktivität anstrebt, kann dies nicht über Leistungsausweitungen geschehen, da in einem solchen Fall zur Finanzierung der Zusatzleistungen der Beitragssatz möglicherweise ansteigen müßte. Gefragt sind daher intelligente Lösungen zur Nutzung von Wirtschaftlichkeitsreserven oder zum Angebot bestimmter krankheitsbezogener oder die Gesundheit unterstützender Informations- und Serviceangebote, die so überzeugend vorgestellt werden können, daß für die Versicherten der BARMER ein Zusatznutzen (added value) klar erkennbar wird, für den sie auch bereit sind, mehr Beitrag zu zahlen. Die frühere Ersatzkassenphilosophie, mit einem günstigen Beitragssatz Leistungen im Bereich zwischen AOKen und privater Krankenversicherung anbieten zu können, muß daher durch ein neues, kundenorientiertes Unternehmensprofil ersetzt werden. Mehr Leistung anbieten ist dann einfach, wenn eine Kasse ohnehin einen niedrigen Beitragssatz hat und trotz einer Beitragssatzanhebung noch immer deutlich unter dem GKV-Durchschnitt liegt - in diesem Fall können z. B. Leistungen aus dem Bereich der unkonventionellen medizinischen Richtungen großzügig angeboten und den Ärztinnen und Ärzten honoriert werden. Wenn eine Kasse aber bereits einen Beitrag über dem GKV-Durchschnitt erheben muß, wird mit der Strategie, Wirtschaftlichkeitsreserven zu erschließen, um mit dem Beitragssatz möglichst nah an den GKVDurchschnitt heranzukommen, gleichzeitig aber ein individuelles Profil als kompetenter Versorgungs- und Beratungsspezialist für die medizinische Behandlung anbieten zu können, um Versicherte zu halten und neu gewinnen zu können, eine Aufgabe sein, die fast der Quadratur des Kreises gleichkommt. Diese Zusammenhänge zur Methodik des RSA sind unbedingt zu bedenken, wenn es um die Bewertung der solidarischen Wettbewerbsordnung in der gesetzlichen Krankenversicherung geht - es ist kein Wettbewerb um Leistungen möglich und auch kein beitragssatztreibender Wettbewerb. Beides würde Kassen wie die AOKen oder die großen Ersatzkassen mit einem Versichertenklientel, das den Durchschnitt der Bevölkerung im Hinblick auf Krankheit und Einkommen abbildet, kurzfristig ruinieren: Der Beitragssatz stiege 
deutlich an, die Versicherten verließen die Kasse, um sich bei einer beitragssatzgünstigeren zu versichern, die Arbeitgeber würden mit Blick auf die Lohnnebenkosten diese Entscheidung, wenn nicht sogar aktiv unterstützen, so doch zumindest freudig begrüßen. Die Beitragssatzanhebungen hätten aber für die jeweilige Kasse eine weitere fatale Konsequenz - möglicherweise wird es hier bald eine gesetzliche Veränderung geben: Diese vom Patienten zu entrichtende Zuzahlung steigt nämlich an, wenn eine Krankenkasse ihren Beitrag wegen der Leistungsausgaben anheben muß: Pro 0,1\% Beitragssatzsteigerung wird die Zuzahlung um weitere 1,-- DM bzw. um $1 \%$ gesteigert. Eine auf Leistungsausgaben beruhende Zuzahlungsanhebung zieht aber ein Sonderkündigungsrecht nach sich, so daß Versicherte in einem solchen Fall zum nächsten Quartal die Kasse verlassen können. Dieser Kopplungsmechanismus "Erhöhung des Beitrages/Erhöhung der Zuzahlung ( $§ 221$ SBG V)" diszipliniert die Kassen zusätzlich, wenn es um Strategien geht, die den sparsamen Weg einer medizinischen Versorgung verlassen, um möglichst attraktiv für neue Versicherte zu sein.

In diesem Zusammenhang ist es eine klare Verzerrung des Wettbewerbs in der GKV, wenn die Wahlmöglichkeiten der Versicherten zwischen den Ersatzkassen und den AOKen obligatorisch eingeräumt werden müssen und mit einem Kontraktionszwang verbunden sind, Betriebs- und Innungskassen (BKK und IKK) sich dagegen entscheiden können, ob sie sich für Versicherte anderer Kassen öffnen. Insbesondere diese beiden Kassenarten versichern ohnehin ein selektiertes Personenklientel - denn wer in den jeweiligen Betrieben nicht seine Arbeitsfähigkeit im Rahmen einer Eingangsuntersuchung nachgewiesen hat, kann auch nicht Mitglied einer BKK oder IKK werden. Dies gilt natürlich nicht für mitversicherte Familienangehörige - dennoch darf die Wirkung dieser privatkrankenkassen-ähnlichen „Risikoselektion" auf den Beitragssatz der genannten Kassen nicht unterschätzt werden. Wenn dann noch Vorteile bei den Verwaltungskosten dadurch hinzukommen, daß Räume und Personal dieser Krankenversicherungen vom Arbeitgeber finanziert werden - ein Vorteil, der etwa 0,5 Beitragssatzpunkte ausmacht -, ist es um so unverständlicher, daß der Gesetzgeber für diese Kassen eine reservatähnliche Nischenpolitik erlaubt hat, die nicht durch die ohne Zweifel vorhandenen Probleme der Wahlfreiheit der Versicherten tangiert wird. Dies ist um so unverständlicher, als bei einer Schließung eines Betriebs mit einer eigenen Betriebskrankenkasse die Er- 
satzkassen oder AOKen natürlich bereitzustehen haben, die nun verwaisten Versicherten in ihre Reihen aufzunehmen. Die Nischenkassen nutzen also alle Vorteile einer Selektion, durch die sie in der Lage sind, günstige Beitragssätze anzubieten, tragen aber letztlich nicht das Risiko, für die Schließung ihrer Kasse besondere Vorsorge treffen zu müssen. Daher sind diese Kassen wegen ihres niedrigen Beitragssatzes auch in der Lage, Leistungshonorare in bestimmten Versorgungsbereichen wie den alternativen Methoden anzubieten, die zwar beitragssatzrelevant sind, innen aber wenig Probleme bereiten, weil sie dennoch beachtlich unter dem durchschnittlichen GKV-Beitragssatz liegen. Damit beeinflussen sie aber möglicherweise insgesamt den Wettbewerb der übrigen Kassen nachhaltig, weil die versuchen müssen, über Verhandlungen mit Ärzten, Krankenhäusern, Apotheken oder anderen Institutionen im Gesundheitssystem über wirtschaftlichere Versorgungsformen als den heute gängigen mit dem Ziel der Ausgabensenkung zu diskutieren, während sich die Nischenkassen mit einem günstigeren Beitragssatz durchaus gewisse Steigerungen ihrer Ausgaben zugunsten großzügiger Honorarzahlungen „leisten" können. Solche Überlegungen können daher daran zweifeln lassen, ob Wahlfreiheit und Risikostrukturausgleich letztlich tatsächlich gleiche Ausgangssituationen für alle Kassen im gegliederten System der gesetzlichen Krankenversicherung herstellen. Während die einen sich mit aller Kraft darum bemühen müssen, trotz einer schwierigen Einnahmensituation die Versorgung der Versicherten über die Nutzung von Wirtschaftlichkeitsreserven mit der unausbleiblichen Folge der Einnahmenverluste bestimmter Anbieter im Gesundheitssystem abzusichern, was den Konflikt zwischen Kassen und Anbietern unausweichlich macht, können andere Kassen im Hochgefühl eines günstigen Beitragssatzes in einem gewissen Rahmen Großzügigkeit an den Tag legen. Wer von den beiden Kassenlagern bei den Anbietern den größeren Bonus hat, ist unschwer zu erraten. Diese positive Wertschätzung wird auch den Versicherten und der Öffentlichkeit nicht verborgen bleiben - Ärztinnen und Ärzte sind nun einmal wichtige Multiplikatoren für die Beurteilung einer Kasse.

Obwohl also die Ausgangssituation für die Wahlkassen nicht unproblematisch erscheint, ist die vom Gesetzgeber vorgesehene Umsetzung von Modellvorhaben oder die Nutzung von Strukturverträgen die einzige Chance, Bewegung in ein erstarrtes und mit vielen Unwirtschaftlichkeiten belastetes Versorgungssystem zu bringen (siehe hierzu auch Ehlers und Leutloff-Simons; 
Schmeinck). Dabei geht es zum einen um zeitlich und räumlich begrenzte Modellvorhaben zur Weiterentwicklung von Versorgungsstrukturen („Strukturmodelle" nach § 63 [1] SGB V) und um eine breitere Anwendung bereits etablierter Ansätze neuer Versorgungsformen im Rahmen der Regelversorgung („Strukturverträge" nach $\S 73$ a SGB V). Die GKV muß den entstandenen Spielraum nutzen - schließlich würden ihre langjährigen Forderungen an die Politik, Instrumente für eine andere Organisation der medizinischen Versorgung zur Verfügung zu stellen, unglaubwürdig, wenn nicht diese Angebote, so eng definiert sie auch sein mögen, in der Praxis zur Anwendung kämen. Das anspruchsvolle Ziel lautet, mit innovativen Versorgungsabläufen im Rahmen veränderter Strukturen Vorteile in der Wirtschaftlichkeit und Attraktivität für die Versicherten derart miteinander verbinden zu können, daß die Position der eigenen Kasse im Beitragssatz und in der Marktakzeptanz optimiert wird. Versicherte werden ihre Kasse zunehmend nach dem Preis-/Leistungsverhältnis auswählen, dies kann beispielhaft in solchen Modellvorhaben angeboten werden. Dieser Ansatz bringt eine Kasse in eine völlig neue Rolle - sie fragt nicht mehr nur Leistungen bei den Anbietern nach, sie wird selber zum Anbieter der medizinischen Versorgung gegenüber ihren „Kunden“. Diese Rolle wird die BARMER kultivieren: Sie will aktiv gestaltender Mittler zwischen (kranken) Versicherten und Leistungserbringern werden und im Sinne einer abgeleiteten Nachfrage die Bedürfnisse der bei ihr versicherten Patienten bei einer notwendigen Krankenbehandlung verantwortungsbewußt in ein optimales Versorgungsangebot umsetzen. Die BARMER will sich mit diesen Anstrengungen für eine Weiterentwicklung der Steuerungsansätze in den Behandlungsabläufen kassenspezifische Wettbewerbsvorteile sichern.

\section{Die Regelungen im einzelnen}

Die neu durch das „Zweite Gesetz zur Neuordnung von Selbstverwaltung und Eigenverantwortung in der gesetzlichen Krankenversicherung (2. GKV-Neuordnungsgesetz)" in das SGB V aufgenommenen $\S \S 63$ bis 65 regeln sowohl das modellhafte Erproben von Modellen zur Versorgung als auch von Leistungen. Danach können Krankenkassen und Verbände zur Erhöhung der Wirtschaftlichkeit und Qualität der Versorgung Modelle durchführen und vereinbaren. Dabei stehen Versorgungs-, Organisations-, Finanzierungs- und Vergütungsformen im Mittelpunkt (Strukturmodelle nach § 63 [1] SGB V). 
Daneben können auch Modellvorhaben zur Verhütung und Früherkennung von Krankheiten, aber auch zur Krankenbehandlung selbst, die Gegenstand des derzeitigen Leistungskatalogs sind, durchgeführt oder vereinbart werden (Leistungsmodelle nach $\S$ 63 [2] SGB V). Vom Bundesausschuß Ärzte und Krankenkassen abgelehnte Leistungen und Fragen der Forschung (Biomedizin, Arzneimittel, Medizinprodukte) können nicht Gegenstand von Leistungsmodellen sein.

Die Inhalte der Modelle (Ziele, Dauer, Bedingungen der Teilnahme) sind in der Satzung zu regeln (§63 [5] 1 SGB V). Damit unterliegen die Modellvorhaben der Genehmigung der jeweils zuständigen Bundes- oder Landesaufsicht.

Die Modellvorhaben sind in der Regel auf 8 Jahre zu beschränken (§ 63 [5] 2 SGB V).

Bei der Vereinbarung und Durchführung kann von den Vorschriften des 4. Kapitels des SGB V und des Krankenhausfinanzierungsgesetzes inklusive der Bundespflegesatzverordnung (BPfIV) abgewichen werden (Suspendierung von § 63 [3] 1 SGB V).

Insgesamt gibt es aber zwei wesentliche Einschränkungen:

- Modelle, die die vertragsärztliche Versorgung berühren, müssen von der jeweiligen KV mitgetragen werden.

- Struktur- und Leistungsmodelle können nur mit zugelassenen Leistungserbringern, also auch nur mit Krankenhäusern im Krankenhausbedarfsplan vereinbart werden ( 63 [1] SGB V). Sog. Einkaufsmodelle oder vergleichbare Optionen sind also nicht möglich.

Modellverträge sind durch die Suspendierung des 4. Kapitels des SGB V kassenartenindividuell und nicht kassenartenübergreifend angelegt. Kassen und Verbände unterschiedlicher Kassenarten können aber durchaus - z. B. mit dem Ziel eines hohen Marktanteils - miteinander kooperieren, es können also strategische Allianzen gebildet werden.

Der Grundsatz der Beitragsstabilität gilt auch für Modellvorhaben ( 63 [3] 1 SGB V). Gegen den Grundsatz der Beitragsstabilität wird insbesondere für den Fall nicht verstoßen, daß durch ein Modellvorhaben entstehende Mehraufwendungen durch nachzu- 
weisende Einsparungen aufgrund im Modellvorhaben vorgesehener Maßnahmen ausgeglichen werden. Für die Kosten zur Durchführung von Strukturmodellen ist ausdrücklich vorgesehen, daß sie z. B. aus dem Honorarbudget eines Krankenhauses herausgerechnet werden, wenn $z$. B. die stärkere Betonung der ambulanten Versorgung in bestimmten Versorgungsbereichen zu einer Entlastung der stationären Ausgaben führt ( 84 [3] SGB V). Sollten sich die Vertragsparteien nicht einig werden, kann das Schiedsamt angerufen werden.

Einsparungen können, soweit sie die Mehraufwendungen überschreiten, auch an die an einem Modellvorhaben teilnehmenden Versicherten weitergeleitet werden (§ 63 [3]) 1 SGB V). Die Form dieser "Rückvergütung" ist nicht geregelt, es können aber $\mathbf{z}$. B. Bonuszahlungen sein. Ein Beitragssatznachlaß, der eine direkte Beteiligung auch der Arbeitgeber bedeuten würde, ist nach derzeitiger Interpretation des Gesetzestextes wahrscheinlich nicht zulässig.

Im Bundesmanteltarif müssen Grundsätze zur Durchführung von Modellvorhaben vereinbart werden ( $\$ 64$ [2] SGB V). Darin muß die gesetzliche Anforderung enthalten sein, daß ein Modellvorhaben dann zustande kommt, wenn mindestens $50 \%$ der Vertragsärzte, die die Voraussetzungen für eine Teilnahme an einem Modellvorhaben erfüllen würden, die Durchführung befürworten (Quorum).

Nach $\S 65$ haben die Krankenkasse oder ihre Verbände eine wissenschaftliche Begleitung und Auswertung der Modellvorhaben im Hinblick auf die Erreichung der Ziele der Modellvorhaben nach $\S 63$ [1 oder 2] nach allgemein anerkannten wissenschaftlichen Standards zu veranlassen. Der von unabhängigen Sachverständigen zu erstellende Bericht über die Ergebnisse der Auswertungen ist zu veröffentlichen.

Die §§ 63-65 bieten demnach einzelnen Kassen und Verbänden die Möglichkeit, die Struktur und Inhalte der medizinischen Versorgung weiterzuentwickeln und alle Aspekte in einem geplanten und kontrollierten Experiment zu untersuchen, die weiter oben in diesem Aufsatz als möglicherweise hinderlich für eine optimale Versorgungsstruktur identifiziert wurden. Durch die Aussetzung des 4. Kapitels des SGB V - und dies umfaßt die $\S \S 69$ bis $140-$ könnten z. B. folgende Hypothesen Basis für Strukturmodelle 
sein, vorausgesetzt die evtl. betroffenen Vertragsärzte hätten im Rahmen der Quorums-Anforderung (s. o.) zugestimmt:

Pauschalierte krankheitsbezogene und sektorübergreifende Honorarsysteme (kombinierte Budgets) führen zu einer geringeren Mengenausweitung als ein Einzelleistungsvergütungssystem, so daß kostentreibende Doppeluntersuchungen oder nicht notwendige Krankenhauseinweisungen unterbleiben. Die in diesem Zusammenhang notwendige Vereinbarung von Leitlinien fördert eine rationale Krankenversorgung (Disease-Management).

Die Steuerung der Inanspruchnahme eines Patienten durch ärztliche Leitstellen verringert ein unnötiges, gleichzeitig aber kostenintensives "Umherirren" des Patienten im System der medizinischen Versorgung ('doctor hopping and clinic shopping').

Der Abschluß von Verträgen, in denen nicht nur die ärztliche Verantwortung für die Indikationsstellung, sondern auch für die ökonomischen Konsequenzen aus dieser Indikationsstellung hat, verringert alle eigenen und induzierten Leistungen und fördert eine kooperative und integrative medizinische Versorgung. Verstärkte Kommunikation, die Erarbeitung von Behandlungsleitlinien und die Patientensteuerung fördern dabei eine für die Patienten optimale und wirtschaftliche Versorgung (Managed Care).

Spezialisierte Leistungen (z. B. in der kardiologischen Diagnostik) können in Krankenhausambulanzen wirtschaftlicher erbracht werden.

Spezielle Verträge mit Hilfsmittelanbietern vereinfachen die Versorgung der Patienten und machen sie überdies wirtschaftlicher.

Es ist möglich, Eigeneinrichtungen zu erproben (Suspendierung von § 140 SGB V). So könnten Krankenkassen eigene Krankenhäuser, Apotheken oder Arztpraxen betreiben, um festzustellen, ob eine solche Versorgungsstruktur sich positiv im Sinne der Wirtschaftlichkeit auswirken würde. So wäre z. B. eine Eigeneinrichtung denkbar, die für die insulinpflichtigen Diabetikerinnen und Diabetiker einer Kasse Blutzuckermeßgeräte und -teststreifen einkauft und sie an die jeweiligen Versicherten verschickt. Diese Hilfsmittel sind nämlich nicht apothekenpflichtig (siehe § 31 [1]) und unterliegen damit nicht dem Versandverbot. 
Ziel solcher Modellvorhaben muß es sein, einen durch Therapieleitlinien abgesicherten, aber auch geforderten Behandlungsablauf bei einer bestimmten Erkrankung optimal umzusetzen - ohne Rücksicht auf egoistische Honorar- oder Umsatzinteressen von Anbietern oder Vertragspartnern im Versorgungssystem. Das Behandlungsziel bei einem insulinpflichtigen Diabetespatienten besteht darin, über eine möglichst gute Patientenführung (regelmäßiges Messen der $\mathrm{HbA} 1 \mathrm{c}-$ Werte, regelmäßige Diagnostik des Augenhintergrundes und des Fußpulses, verständliche Schulungsmaßnahmen) Spätfolgen zu vermeiden. Daher müssen hausärztliche Praxis, Diabetes-Schwerpunktpraxis und Akutkrankenhaus eng auf der Basis eines wissenschaftlich etablierten Behandlungskonzeptes zusammenarbeiten. Eine von allen Beteiligten akzeptierte Qualitätssicherung zeigt die Erfolge, aber auch die Defizite der Behandlung auf. Bei Defiziten muß vorher geregelt sein, welche Interventionen auf welcher Ebene des Versorgungssystems erforderlich werden. So ist es unwirtschaftlich, Patienten zur Schulung in ein Akutkrankenhaus einzuweisen, sinnvoller ist die Organisation von Schulungsmaßnahmen durch die DiabetesSchwerpunktpraxis in einem Schulungszentrum. Es müssen auch Second-Opinion-Strukturen vereinbart werden. Vor einer Einweisung eines Diabetespatienten durch einen Hausarzt in ein Krankenhaus sollte eine obligatorische Konsultation zwischen Hausarzt und Schwerpunktarzt stattfinden, um eventuell doch die teuren Krankenhausaufenthalte vermeiden zu können. So hat eine Analyse der Krankenhausliegezeiten von Diabetikern im Raum Wuppertal ergeben, daß die mittlere Verweildauer von rund 17,1 Tagen pro Jahr auf etwa 11,5 Tage hätte verringert werden können, wenn die im ambulanten Bereich durchführbare Versorgung auch wirklich dort stattgefunden hätte. Ärzte überweisen derzeit aber lieber ins Krankenhaus als an einen Spezialisten, weil sie aus dem Krankenhaus ihre Patienten sicher zurückbekommen, ein Spezialist aber womöglich den Patienten behält. Konkurrenzdruck als Grund für eine unnötig und teure Krankenhauseinweisung!

Insgesamt ist ohnehin zu unterstreichen, daß die Strukturmodelle die Verringerung der in Deutschland nach Expertenmeinung auffällig langen Verweildauern zum Ziel haben. Von den rund 235 Mrd. DM der GKV-Leistungsausgaben entfielen 1996 immerhin $78 \mathrm{Mrd}$. DM auf den stationären Sektor - mehr als für die ambulante Versorgung (ärztliches Honorar 39,3 Mrd. DM, Arzneimittel 33,5 Mrd. DM). Bei der derzeitigen mittleren Verweildau- 
er im Krankenhaus von rund 12 Tagen pro Fall entspräche eine Reduktion von 2 Tagen bei gleicher Fallzahl einer Verringerung des Beitragssatzes von nahezu einem Prozentpunkt - ein Ziel, das mit Blick auf die international bekannten Verweildauern mit ungefähr 7 Tagen bei einer gleichzeitigen, besseren Organisation des ambulanten Versorgungsbereiches durchaus erreichbar erscheint. Untersuchungen weisen darauf hin, daß durch längere Verweildauern als medizinisch gerechtfertigt die durchschnittliche Verweildauer 2,6 Tage hätte niedriger sein können, was etwa 85.000 überflüssiger Betten und 26 Millionen überflüssiger Pflegetage entspricht (OECD). Gleichzeitig wird auch darauf hingewiesen, daß die Fallzahlen in Deutschland im europäischen Vergleich um $15 \%$ über dem Durchschnitt liegen - auch hier sollte durch eine stärkere Berücksichtigung der Behandlungsmöglichkeiten im ambulanten Sektor und durch bessere Angebotsstrukturen, z. B. durch 24 Stunden am Tag erreichbare "Anlaufpraxen", eine Reduktion dieser auffällig hohen Fallzahlen möglich sein. Die Strategien, soviel stationär wie nötig und soviel ambulant wie möglich bzw. ambulant vor stationär, sind daher Basis für Wirtschaftlichkeitsreserven in allen Strukturmodellen, die in der GKV geplant werden, und die vorzugsweise chronische Erkrankungen (wie z. B. Diabetes, Asthma, rheumatoide Arthritis, Hypertonie u. a.) oder plötzlich auftretende Krankheitsereignisse (z. B. Schlaganfall) einbeziehen.

Leistungsmodelle, die ebenfalls nach $\S 63$ durchgeführt werden können, beziehen sich vor allem auf Therapieoptimierungsmöglichkeiten bei einzelnen Erkrankungen. So führen die Ersatzkassen ein Modellvorhaben zur Anwendung der ambulanten Balneophototherapie bei Psoriasis-Patientinnen oder -Patienten durch, die Behandlungsaufenthalte am Toten Meer überflüssig machen sollten, da entsprechende ambulante Angebote gleiche Therapieerfolge ermöglichen. Solche Therapieoptimierungsmodelle sind auch in anderen Indikationsbereichen (z. B. Onkologie, Allergien, Asthma usw.) denkbar. Da nach § 65 alle Modellvorhaben nach dem allgemein anerkannten Kenntnisstand zu begleiten und zu evaluieren sind, könnten Leistungsmodelle auch im Sinne der Versorgungsforschung genutzt werden, mit der unterschiedliche therapeutische Ansätze miteinander verglichen und zu einer aussagefähigen Optimierung eines Behandlungsstandards führen könnten. 
Entgegen den $\S \S 63-65$ bietet der $\S 73$ a keine Handlungsmöglichkeiten für einzelne Kassen. Vielmehr wird hier auf Vereinbarungen zwischen den Landesverbänden der Krankenkassen und den Verbänden der Ersatzkassen mit den kassenärztlichen Vereinigungen abgestellt. Damit sind neben den zeitlich befristeten Strukturmodellen nach $\S 63$ (1) SGB V mit dem 2. NOG auch Möglichkeiten geboten, neue Versorgungs- und Vergütungsstrukturen auf Dauer in den Gesamtverträgen nach $\S 83 \mathrm{zu}$ vereinbaren. In Frage kommen hierfür z. B. Hausarztmodelle, 'vernetzte Praxen' oder kombinierte Budgets. Der Gesetzgeber wollte damit bereits erfolgreichen Versorgungsinnovationen zur dauerhaften Umsetzung verhelfen. Es muß jedoch bezweifelt werden, ob die erwähnten neuen Versorgungskonzepte bereits ihren Erfolg nachweisen konnten. So scheiterten z. B. bislang alle Ansätze, den $\S 73$ zur Basis einer vertraglichen Regelung zu machen, mit dem die koordinierenden Aufgaben der hausärztlichen Versorgung herausgestellt werden, am Widerstand der fachärztlichen Fraktionen in den KVen. Und auch die Konzepte der kombinierten Budgets oder Praxisnetze haben in der Praxis bislang entweder nicht überzeugt oder sind noch nicht abgeschlossen: So gibt es im „Praxisnetz Berliner Ärzte und BKK“, zu dem kürzlich auch die Techniker-Krankenkasse als Kooperationspartner gestoßen ist, zwar rund 250 haus- und fachärztlich tätige Ärzte, bislang aber zu wenig Patientenverläufe, an deren Versorgung ablesbar wäre, wie sich ein solches Modell bewährt. Das vielversprechend begonnene Projekt der "Medizinischen Qualitätsgemeinschaft Rendsburg", das die Ersatzkassen vereinbart haben, arbeitet dagegen erfolgreich, ist aber noch nicht abgeschlossen. Insofern beziehen sich die im $\S 73$ a angesprochenen Strukturverträge auf Versorgungsstrukturen, deren positiver Einfluß in der Praxis noch nicht als bestätigt gelten kann. Es wird daher noch Zeit und Entwicklungskosten erfordern, um das programmatische Konzept der „vernetzten Praxen mit kombinierten Budgets" und die damit verbundenen Organisationsstrukturen und Qualitätssicherungsmaßnahmen zu entwickeln. Eine bloße Anschubfinanzierung kann hier nicht weiterhelfen. Außerdem ist eine Regelversorgung im Sinne vernetzter Praxen gerade auch im Hinblick auf die Versicherten noch nicht sinnvoll: Wenn die "Marktreife" solcher Systeme noch keineswegs als evaluiert gelten kann, ist Versicherten nicht zuzumuten, sich ohne Wahlmöglichkeit in einem solchen Netz behandeln zu lassen. Der sonst üblichen und hochgehaltenen freien Arztwahl müßte dann auch die freie Netzwahl entsprechen. Wenn aber Strukturverträge nicht mehr im Sinne von Wett- 
bewerbsmodellen klassifiziert werden können, weil sie als geregelte Versorgung angeboten werden, wäre diese Wahlmöglichkeit dahin - es gäbe als Ausweg nur noch den Wechsel in eine andere Kassenart vor Ort, die sich an solchen Strukturverträgen nicht beteiligt hat.

\section{Auswirkungen der Modellvorhaben und Strukturverträge für pharmazeutische Hersteller}

Grundsätzlich steht bei allen Modellvorhaben nach § 63 SGB V die optimierte Steuerung der Behandlungsabläufe im Vordergrund mit dem wichtigen Ziel, den ambulanten Bereich soweit wie möglich zu fördern, um damit Einsparungen im stationären Bereich zu erreichen. Hierzu werden in der Vergütungsform der Strukturmodelle auch Bonusregelungen vereinbart (incentives), die den niedergelassenen Ärztinnen und Ärzten entweder "Beteiligungen" an den eingesparten Kosten bei der stationären Behandlung oder pauschalierte Honorare für medizinisch vertretbare Vermeidungen einer Einweisung zusichern.

Die stärkere und "bewußte" Betonung des ambulanten Bereichs verlangt auch nach einem anderen Umgang mit Versorgungsabläufen als derzeit: So wird die Vermeidung des „Reparaturbetriebs" Krankenhaus belohnt, z. B. bei der "Entgleisung" einer ambulant durchaus erfolgreich durchführbaren Behandlung von Diabetes oder Herzinsuffizienz, die Vermeidung des stationären "Wochenendservices", wenn nämlich niedergelassene Ärztinnen oder Ärzte ab Freitagnachmittag nicht mehr zur Verfügung stehen oder die Vermeidung "Abschiebebahnhof" Klinik, die oftmals dann in Anspruch genommen wird, wenn teure Patienten nicht mehr in das Arzneimittelbudget passen, und damit die indizierte, wirksame und qualitätsgesicherte ambulante Versorgung gefördert.

Erreicht und unterstützt wird dies im Rahmen einer strukturierten Versorgung (Therapieleitlinien, -empfehlungen) u. a. durch den indizierten Einsatz wirksamer und ambulant anwendbarer Pharmakotherapien (z. B. bei Asthma, Diabetes, Herzinsuffizienz, Parkinson, Krebs, Depression, Ulkus u. ä.). Arzneimittel werden damit stärker als bislang zur Substitution kostenintensiver stationärer Behandlungen herangezogen - Strukturmodelle sparen mit Arzneimitteln, nicht an Arzneimitteln.

Aus diesem Grund sind Strategien der Arzneimittelbehandlung besser als bislang den unterschiedlichen Ebenen von Therapie- 
leitlinien anzupassen, mehr als bislang zählt die "Software" Arzneimittelinformation als added value, die "Hardware" Arzneimittel wird eher austauschbar. Modelle sollen daher die Frage beantworten, welche Versorgungsebene im Rahmen welcher Struktur mit welchen effektiven Mitteln die größtmögliche Effizienz bietet.

Zu den effizienten Mitteln der Krankenbehandlung bieten sich ohne Zweifel Arzneimittel als wichtige "Handwerkszeuge des Arztes" an, wenn sie in dem beanspruchten Indikationsgebiet ihre Wirksamkeit nachgewiesen haben, also effektive Therapeutika darstellen. Die Chance auf Berücksichtigung in Leitlinien haben daher auf Dauer nur wirklich innovative bzw. in ihrem Nutzen bestätigte Mittel. Daneben spielen allenfalls echte Placebos eine Rolle.

"Undekuvrierte“ Placebos (zweifelhaft wirksame Mittel) werden unter diesen therapeutischen Herausforderungen als wirksame Substitution für Krankenhausaufenthalte versagen - ihre Bedeutung wird daher besonders bei chronischen Erkrankungen zurückgehen. Zweifelhaft wirksame oder nicht sinnvoll kombinierte Arzneimittel werden daher bei künftigen Regelungen in der GKV noch weiter als schon bisher zurückfallen. Aktualisierte Negativlisten oder die Gruppe 3 der Richtgrößenregelung werden solche Mittel in ihrer Bedeutung noch stärker zurückdrängen.

Daher bieten gerade Modellversuche eine gute Vermarktungschance für wirklich innovative und wirksame Mittel mit einem „Krankenhausverhinderungspotential" - therapeutisch vielversprechende Forschungsergebnisse sind somit ein gutes Kapital für die Zukunft einer strukturierten, evidence-based-orientierten Versorgung. Disease-Management- oder Risk-Sharing-Konzepte, mit denen Ärzten zusätzlich eine Sicherheit für die Wirksamkeit einer ambulanten Therapie gegeben wird, weil die jeweiligen Hersteller innen den Bonusverlust wegen einer dennoch notwendigen Krankenhauseinweisung absichern, könnten ein übriges für die Akzeptanz sinnvoller Arzneimittel mit Substitutionspotential tun. Und daß Arzneimittel stationäre Behandlungen einschränken oder ganz überflüssig machen können, haben viele Beispiele belegt. Erinnert sei an die $\mathrm{H}_{2}$-Antagonisten zur Vermeidung von $\mathrm{Ul}$ zeraoperationen oder an die modernen Antidepressiva oder Neuroleptika, die langzeitige stationäre Psychiatrieaufenthalte bei vielen Patienten unnötig machten. 
Für die pharmazeutischen Hersteller von wirksamen und/oder wirklich innovativen Arzneimitteln sind daher im Rahmen neuer Versorgungsstrukturen erweiterte Absatzchancen möglich und wahrscheinlich. Dies ist sicherlich auch der Grund, warum vor allem die forschenden Arzneimittelhersteller die Kooperation mit Krankenkassen anstreben. Wenn schon vertragliche Regelungen zwischen den Partnern der Strukturmodelle mit den pharmazeutischen Herstellern gesetzlich nicht vorgesehen sind - und hier widerspreche ich ausdrücklich SMIGIELSKI, der eine solche Möglichkeit im $\S 64$ [1] entdeckt haben will, obwohl pharmazeutische Hersteller im SGB V nicht zu den Leistungserbringern gerechnet werden -, so kann doch die organisatorische Mitgestaltung eines Strukturmodells einen wichtigen Erkenntnisgewinn „aus erster Hand" bedeuten. Zudem könnten Instrumente wie Erhebungsoder Dokumentationssysteme, die einige Firmen im Rahmen ihrer Disease-Management-Konzepte erstellt haben, auch für Ärzte und Krankenkassen eine nützliche Unterstützung darstellen, vorausgesetzt, diese Konzepte haben sich von einer präparateorientierten Marketingstrategie gelöst. Zur Beruhigung vieler Hersteller muß aus Sicht der GKV leider auch festgehalten werden, daß in den Strukturmodellen, übrigens auch in den Strukturverträgen, nicht von den bestehenden arzneimittelrechtlichen und apothekenrechtlichen Vorschriften sowie vom Leistungskatalog der Krankenversicherung, wie er im 3. Kapitel festgeschrieben ist, abgewichen werden kann. Versandapotheken, Veränderungen der Preisspannenverordnung oder Positivlisten können daher nicht "Untersuchungsgegenstand" von Modellvorhaben und Strukturverträgen sein.

Während die §§ 63-65 SGB V vor allem Chancen für die innovativen pharmazeutischen Hersteller bieten, bestehen im Zusammenhang mit den Strukturverträgen nach $\S 73$ a durchaus Risiken (ÄRZTEBLATT). Entsprechende Verträge zwischen verschiedenen Ortskrankenkassen und Kassenärztlichen Vereinigungen sehen nämlich Honorarerhöhungen vor, wenn im Arzneimittelbereich Einsparungen vorgenommen werden. In Berlin sind solche Vereinbarungen abgeschlossen worden, ohne die Ärzte zur Teilnahme an Qualitätssicherungsmaßnahmen zu verpflichten. Solche nichtqualitätsgesicherten und letztlich intransparenten Bonusmodelle in der Arzneimittelversorgung sind aus meiner Sicht abzulehnen und als der Therapiequalität möglicherweise abträgliche Bereicherungsangebote für Ärztinnen und Ärzte zu diskreditieren. Wenn solche Regelungen nicht im Rahmen sektorübergrei- 
fender Strukturverträge vereinbart werden, können sie letztlich kontraproduktiv wirken: Teure Patientinnen und Patienten werden ohne Einbuße für die Ärzte in das Krankenhaus abgeschoben, damit innen der Bonus erhalten bleibt. Kassen, die solchen Regelungen zustimmen, haben das Ziel der neuen Versorgungsstrukturen nicht verstanden - die BARMER hat sich gegen solche unqualifizierten Sparkonzepte ausgesprochen.

\section{Ausblick}

Die Strukturmodelle nach § 63 SGB V bieten der GKV Ansätze für Suchprozesse nach besseren Versorgungslösungen im Rahmen eines wettbewerblichen Rahmens. Gestützt wird dieser Wettbewerb auch dadurch, daß nicht nur Kassenarten, sondern auch einzelne Kassen die Möglichkeit wahrnehmen können, sich im Markt zwischen Wahlfreiheit und Risikostrukturausgleich mit intelligenten Angeboten für ihre Versicherten zu profilieren und sich im Rahmen einer sonst für eine einzelne Kasse nicht möglichen Vertragsgestaltung als kompetenter Versorgungsspezialist zu positionieren. Hierdurch werden in unserem gegliederten Krankenversicherungssystem dynamische Wettbewerbsprozesse in Gang gesetzt, eine Einheitskasse würde Einheitslösungen produzieren, die solchen dynamischen Prozessen gegenüber wenig "anfällig" wäre. Die angestrebten Vernetzungen der einzelnen Behandlungsebenen im Versorgungssystem machen übrigens auch deutliche Veränderungen innerhalb der einzelnen Kassen notwendig: Die für viele Unwirtschaftlichkeiten im System verantwortliche Sektorierung („Versäulung“) der medizinischen Versorgung hat nämlich ein Spiegelbild in der Organisation der Kassen gefunden - auch hier ist während der vergangenen Jahre die sektorale Verwaltung der ambulanten Versorgung, des stationären Bereiches, der Arzneimittelversorgung oder der Rehabilitation neben der Versorgung mit Heil- und Hilfsmitteln oder anderen Sparten vertikal erstarrt. Horizontale Vernetzungen sind wie das Verständnis für die Rolle eines Gestalters für medizinische Versorgung im Aufbau begriffen. Diese intern notwendige Weiterentwicklung der Organisationsstruktur einzelner Kassen ist der vielleicht überraschende Nebeneffekt der Neuregelung für Strukturmodelle - wie "draußen" im Versorgungssystem müssen auch "drinnen" Versorgungsabläufe, nicht Versorgungsstationen die Vernetzung der einzelnen Sektoren fördern. 
Die Krankenkassen werden unter dem Eindruck von Einnahmenund Finanzierungsproblemen vor allem die Ergebnisse aus Strukturmodellen nutzen wollen, um über die Nutzung der ohne Zweifel vorhandenen Wirtschaftlichkeitsreserven die Zukunftsfähigkeit der solidarisch finanzierten gesetzlichen Krankenversicherung abzusichern. Die Anbieter im System, für die eine Krankenversicherung für etwa $90 \%$ der deutschen Bevölkerung ein wichtiger Standortvorteil im Hinblick auf einen kalkulierbaren Arbeits-, Absatz- und Investitionsmarkt gegenüber anderen Ländern mit weniger klaren Versicherungsstrukturen wie z. B. den USA darstellt, sind aufgerufen, in verantwortlicher Kooperation mit den Krankenkassen nach Lösungen zu suchen, die auch in Zukunft tragen und unser Krankenversicherungssystem nicht (be)schädigen. Einkommens- und Umsatzverluste sind zwar in einzelnen Sektoren nicht vermeidbar, vertraglich induzierte Veränderungen gehen meist zu Lasten Dritter. Bislang gingen sie aber zumeist zu Lasten der Patienten mit der Folge, daß die Privatisierung des Risikos Krankheit fortschritt. Klagen der Anbieter im Versorgungssystem sind daher nicht angebracht. Sie sollten also - mit J. F. Kennedy - nicht fragen, was die GKV für sie tun kann, sie sollten sich lieber fragen, was sie zum Fortbestand der GKV beitragen können!

\section{Literatur}

ÄRZTEBLATT (1997) VFA will aktive Rolle bei Strukturverträgen. Dtsch. Ärztebl. 94, 40: B-2076

ÄRZTE-ZEITUNG (1997) Wissenschaftsjournal Forschung und Praxis Nr. 240: 25-26

Bruckenberger E (1996) Situation der Herzchirurgie 1995 in Deutschland. 8. Bericht des Krankenhausausschusses der Arbeitsgemeinschaft der Leitenden Medizinalbeamtinnen und Medizinalbeamten (AGLMB). Hannover (Niedersächsisches Sozialministerium). S. 24

Donner-Banzhoff N (1994) Allgemeinmedizin in Großbritannien und Deutschland. Die Auswirkungen verschiedener Vergütungssysteme auf die Qualität präventiver Versorgung. LIT Verlag, Hamburg, Münster.

Ehlers APF, Leutloff-Simons UH (1995) Reformmodelle des Deutschen Gesundheitssystems in der Übersicht. Recht und Politik im Gesundheitswesen 2: 63-77. Springer-Verlag. Berlin, Heidelberg.

FRANKFURTER RUNDSCHAU: Interview am 23. August 1997. S. 8

Gerhardt P (1996) Diagnostische Radiologie im Spektrum der Kostenentwicklung in der Medizin. Radiologie 36: 270-278 sowie KISTLER E (1996) 
Lean Service im Krankenhaus: Ausgliederung von Krankenhausbehandlungen und Telemedizin. Manuskript, INIFES. Augsburg.

Glaeske G (1997) Einheitlicher Bewertungsmaßstab (EBM) versus Vergütung primärärztlicher Tätigkeit durch eine differenzierte Pauschale. In: Helmich $P$ et al. (Hrsg.) Primärärztliche Patientenbetreuung. Schattauer Verlag. 321-331

Lange S, Richter K, Köbberling J (1994) Knochendichtemessung zur Früherkennung der Osteoporose - Entscheidungshilfe oder Selbstzweck. Argument-Jahrbuch 22: $56-78$

OECD (1997) OECD Wirtschaftsberichte 1996-1997, Deutschland.

Schmeinck W (1997) Modellvorhaben und Strukturverträge. Brennpunkt Gesundheitswesen, hrsg. von der Schering AG. 8: 16-21. Berlin

Schwabe U, Paffrath D (1996) Arzneiverordnungs-Report '96. G. FischerVerlag. Stuttgart Jena

Smigielski E (1997) Sektorübergreifende Versorgungsformen und Chancen für die pharmazeutische Industrie. Pharm. Ind. 59, 5: 369-373.

STATISTICS (1997) Alle Zahlen in der Einleitung stammen, sofern nichts anderes angegeben ist, aus Statistics ' 97 , herausgegeben vom Verband Forschender Arzneimittelhersteller (VFA). Bonn.

Wimmer E (1997) Morphin - Pharmaklassiker mit Zukunft. Münch. med. Wschr. 139 (Spezial). 16-18 


\section{Strukturverträge und Modellvorhaben aus kassenärztlicher Sicht}

Lothar Krimmel

\section{Vorbemerkung}

„Nichts auf der Welt ist stärker als eine Idee, für welche die Zeit gekommen ist." Diese aphoristische Beschreibung menschlicher Begeisterungsfähigkeit, die in der wechselvollen Geschichte der Menschheit bereits des öfteren ihre Bestätigung gefunden hat, ist auch geeignet, die aktuellen Aktivitäten von Ärzten und Krankenkassen bei der Umgestaltung der Versorgungsstrukturen in Richtung auf vernetzte Systeme zu beschreiben. In allen Teilen der Republik machen sich Ärzte und Krankenkassen auf den Weg, neue Versorgungsformen in der Praxis anzuwenden. Daß dabei auf beiden Seiten teilweise sehr unterschiedliche Motive zugrunde liegen, stört angesichts der derzeitigen Aufbruchstimmung offensichtlich nur die wenigsten.

Obwohl die Diskussion um vernetzte Praxen, Strukturverträge und Modellversuche in Deutschland noch relativ jung ist, kann die Literatur hierzu bereits jetzt schon kaum noch überblickt werden. Ich möchte mich in meinen Ausführungen deswegen auch nicht auf das weite Feld der Grundsatzdiskussionen oder detaillierter Einzelanalysen einlassen, sondern nur einige Aspekte aufgreifen, die mir in der aktuellen Diskussion teilweise ein wenig zu kurz zu kommen scheinen.

\section{Systematische Einordnung der Diskussion}

Es darf nicht übersehen werden, daß zu den Hauptmotiven für die Einführung Managed-Care-artiger Versorgungsstrukturen auch in Deutschland die Finanzierungskrise des Gesundheitswesens gehört. In Abbildung 1 ist dargestellt, daß die wachsende Inanspruchnahme medizinischer Leistungen auf der einen Seite und die zunehmende ökonomische Austrocknung einer ausschließlich lohngebunden finanzierten gesetzlichen Krankenversicherung andererseits nicht mehr in Deckung zu bringen sind. Es wäre fatal zu glauben, daß Managed Care geeignet sein könnte, diesen grundsätzlichen systematischen Fehler in der Finanzierung der Leistungen einer gesetzlichen Krankenversicherung zu beheben. 
Aus diesem Grunde muß einer Erweiterung der Finanzierungsbasis der gesetzlichen Krankenversicherung mindestens soviel Aufmerksamkeit gewidmet werden wie einer Effizienzsteigerung im Bereich der eigentlichen medizinischen Versorgung.

Abb. 1

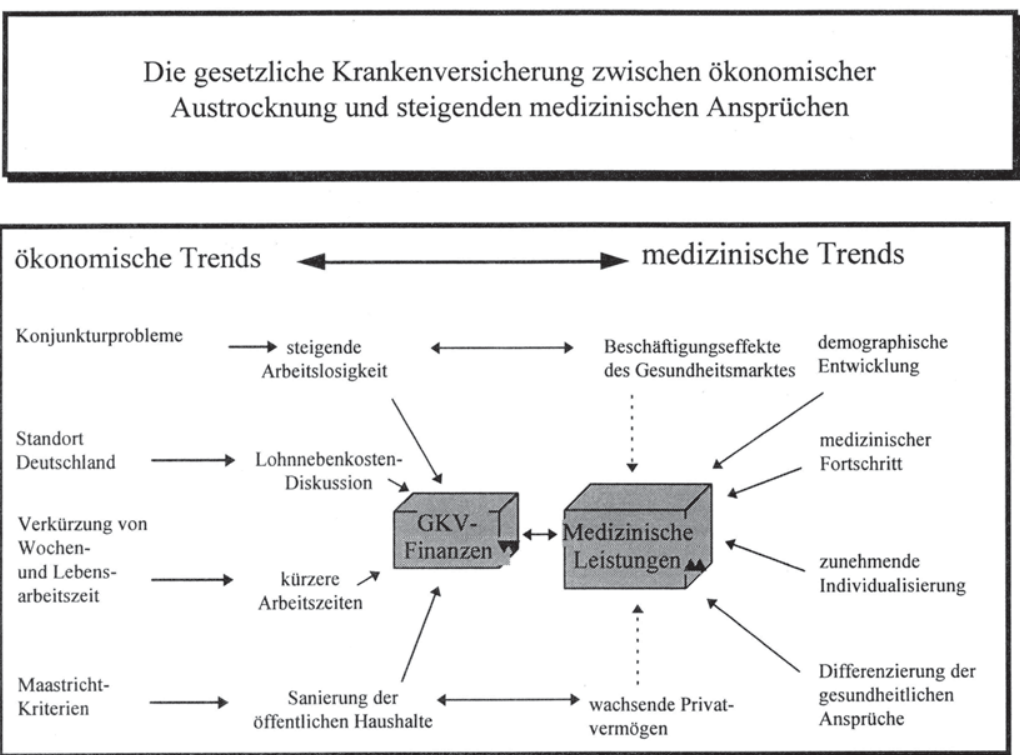

Es müssen sich darüber hinaus alle Beteiligten im klaren sein, daß die Implementierung von Managed-Care-Ansätzen in das deutsche Gesundheitswesen unweigerlich mit Leistungseinschränkungen für die Mitglieder der gesetzlichen Krankenkassen verbunden sein wird. Dabei mögen diese Einschränkungen über den Managed-Care-Prozeß für die gesundheitspolitisch Verantwortlichen komfortabler sein als politisch getragene explizite Leistungsausschlüsse, zumal implizite Leistungseinschränkungen durch die Ebene einzelner Ärzte oder „Arztnetze" mit Budgetverantwortung viel weiter gehen können, als dies im Rahmen einer öffentlichen Diskussion zu expliziten Leistungsausschlüssen jemals möglich wäre.

In Abbildung 2 ist dargestellt, daß sich das Managed-CareInstrument unter dem Aspekt der Verbesserung der „Prozeßeffizienz" somit nahtlos in den ablaufbezogenen Kreislauf möglicher Steuerungsansätze zur effizienten Finanzallokation im Gesund- 
heitswesen einordnet. Ausgehend von der Beschränkung der Zielvorgaben auf die prioritären Gesundheitsziele und die Einschränkung des Leistungskataloges auf der Grundlage von Evidence-Based-Medicine reicht das Spektrum dieser Steuerungsansätze über das Managed-Care-System schließlich bis zum Schlagwort der Ergebnisorientierung ärztlichen Handelns. Es mag sein, daß der unauflösbar scheinende Widerspruch zwischen wachsendem Bedarf und sinkendem Deckungspotential keine anderen Auswege zuläßt; es wäre jedoch fatal, wenn in der öffentlichen Diskussion verschwiegen würde, daß alle diese Ansätze mit der "Freibiermentalität" im Gesundheitswesen, die den Bürgern bis heute aus der großen Koalition von Gesundheitspolitikern, Krankenkassen und Medien vermittelt wird, auch nicht einmal mehr ansatzweise in Deckung zu bringen sind.

Abb. 2

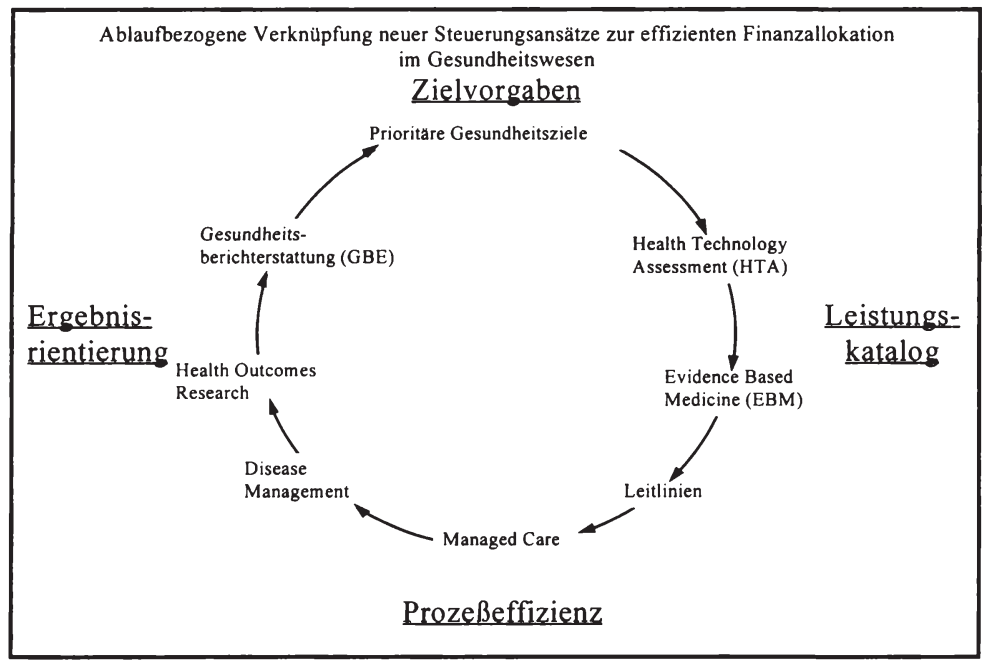

\section{Gesetzliche Grundlagen von Modellvorhaben und Strukturverträgen}

Die Kassenärztliche Bundesvereinigung hat sich im Gesetzgebungsverfahren zum 2. GKV-Neuordnungsgesetz nachhaltig dafür eingesetzt, mit den Modellvorhaben nach $\S 63 \mathrm{ff}$. SGB $V$ und insbesondere den Strukturverträgen nach $\S 73$ a SGB V die rechtlichen Möglichkeiten zur Weiterentwicklung der Versorgung entscheidend zu verbessern. Der Gesetzgeber hat damit in der 
Tat die vertraglichen Möglichkeiten der gemeinsamen Selbstverwaltung erweitert und dabei - wie Abbildung 3 zeigt - die Zuständigkeiten von KBV und Kassenärztlichen Vereinigungen im Rahmen dieser vertraglichen Weiterentwicklung der Versorgungsstrukturen klar festgelegt. Während die Grundsätze zur Durchführung der Modellversuche und die Rahmenverträge zu Inhalt und Durchführung von Strukturverträgen Aufgabe der KBV und der Spitzenverbände der Krankenkassen sind, werden die Vereinbarung von Modellvorhaben selbst sowie die Vereinbarung der Versorgungsverträge für Hausärzte und vernetzte Praxen auf der Ebene der Kassenärztlichen Vereinigungen und der Krankenkassen bzw. ihrer Landesverbände wahrgenommen. Eine Ausnahme in dieser ansonsten klaren Gliederung ist nur durch die rechtlich und vertragspolitisch interessante Kompetenz der Kassenärztlichen Bundesvereinigung gegeben, selbst Modellvorhaben mit einzelnen Krankenkassen oder Krankenkassenverbänden vereinbaren zu können.

Abb. 3

Zuständigkeiten von KBV und Kassenärztlichen Vereinigungen bei der vertraglichen Weiterentwicklung der Versorgungsstrukturen

\begin{tabular}{|l|l|l|}
\hline & \multicolumn{1}{|c|}{ KBV } & \multicolumn{1}{c|}{ KVen } \\
\hline $\begin{array}{l}\text { Strukturverträge } \\
(\S 73 \text { a SGB V) }\end{array}$ & $\begin{array}{l}\text { - Rahmenverträge zu Inhalt } \\
\text { und Durchführung }\end{array}$ & $\begin{array}{l}\text { - Versorgungsverträge } \\
\text { für Hausärzte und } \\
\text { vernetzte Praxen }\end{array}$ \\
\hline $\begin{array}{l}\text { Modellversuche } \\
(\S 63 \text { ff. SGB V) }\end{array}$ & $\begin{array}{l}\text { - Vereinbarung von Modell- } \\
\text { vorhaben } \\
\text { Grundsätze zur Durchfüh- } \\
\text { rung }\end{array}$ & $\begin{array}{l}\text { Vereinbarung von } \\
\text { Modellvorhaben }\end{array}$ \\
\hline
\end{tabular}

Die rechtlichen Rahmenbedingungen für die Modellvorhaben nach $\S 63 \mathrm{ff}$. SGB V sind in Abbildung 4 dargestellt. Unter dem Aspekt der Zielsetzung einer strukturellen Weiterentwicklung der gesetzlichen Krankenversicherung ragen unter diesen $\mathrm{Be}$ stimmungen zwei Aspekte heraus: zum einen die Möglichkeit der Gewährung eines Versichertenbonus und zum anderen der Auftrag an KBV und Spitzenverbände der Krankenkassen, in den Bundesmantelverträgen auch Regelungen zu treffen, wonach ein Modellvorhaben auch dann zustande kommt, wenn ein "Quorum" von mindestens $50 \%$ der in Frage kommenden Vertragsärzte die Durchführung des Modellvorhabens befürworten. KBV und Spit- 
zenverbände der Krankenkassen werden die entsprechenden Regelungen im ersten Halbjahr 1998 treffen. Dabei sind im Hinblick auf das angesprochene Quorum unsinnige Regelungen zu vermeiden. Beispielsweise wäre es sinnlos, einen Modellversuch zur künstlichen Befruchtung in einer Mittelstadt davon abhängig zu machen, daß der einzig hierfür in Frage kommende Arzt "zu $50 \%$ " den Modellversuch befürwortet.

Abb. 4

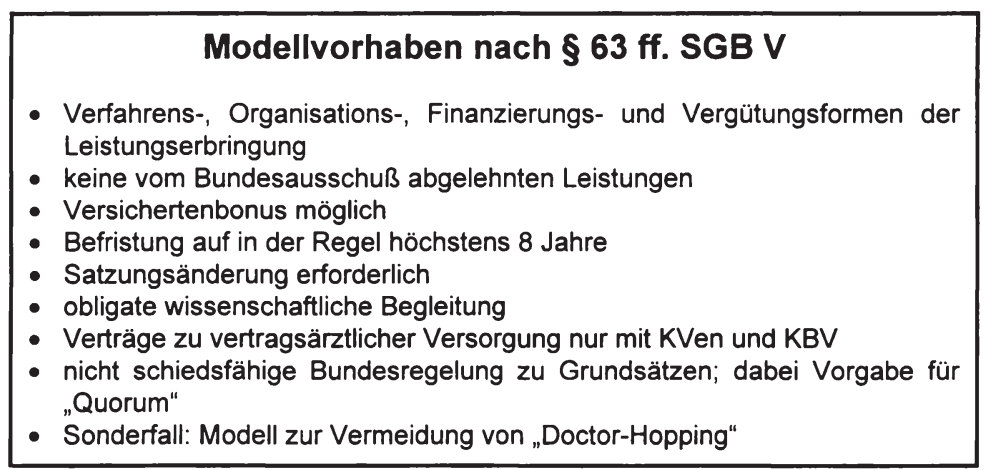

In Abbildung 5 sind die gesetzlichen Bestimmungen zu den Strukturverträgen nach $\S 73$ a SGB V dargestellt. Dabei muß zunächst darauf hingewiesen werden, daß $§ 73$ a SGB $V$ die rechtliche Grundlage für die Einführung der derzeit international am stärksten diskutierten Entwicklungsoptionen bietet, nämlich zum einen der Installierung eines hausärztlichen Primärarztes mit „Fundholder"-Kompetenz und zum anderen des Aufbaus vernetzter Praxen mit Budgetverantwortung auch für veranlaßte Leistungen. Eine Schwäche dieser Regelung ist allerdings, daß im Rahmen dieser Strukturverträge - im Gegensatz zu den Modellversuchen nach § $63 \mathrm{ff}$. SGB V - kein Versichertenbonus gewährt werden darf. Auf diese Weise dürfte die freiwillige Teilnahme von Versicherten die größte Hürde für die Übertragung einer Budgetverantwortung an den Hausarzt oder an vernetzte Praxen sein. Es ist aus dem Grund wahrscheinlich, weil der Aufbau einer "real existierenden Versorgung" auf vertragliche Mischformen der Modellversuche nach $\S 63 \mathrm{ff}$. SGB V und von Strukturverträgen nach $\S 73$ a SGB V - unter Einschluß der Möglichkeit eines Versichertenbonus - gestützt wird. 
Abb. 5

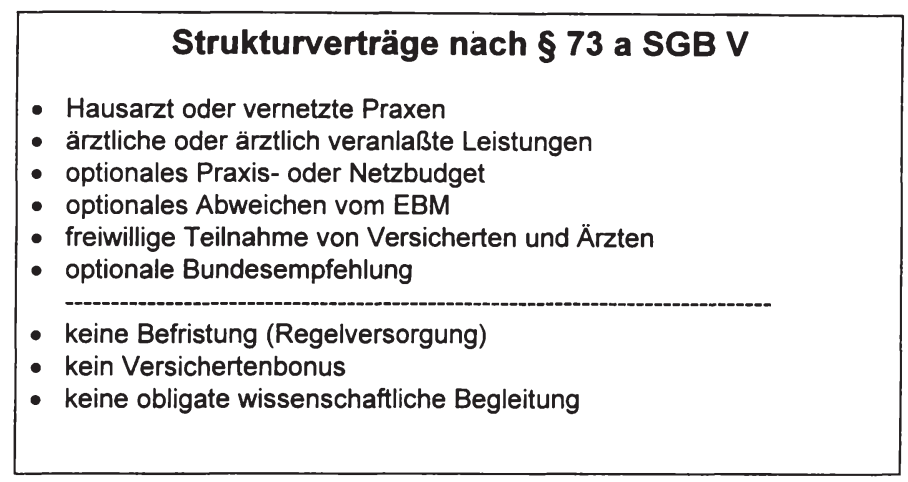

An dieser Stelle werden auch die eingangs angesprochenen Differenzen in den Motiven von Kassenärzten und Krankenkassen deutlich. Für die Kassenärzte und auch für die kassenärztliche Selbstverwaltung kommt es im wesentlichen darauf an, durch vernetzte Praxen die entscheidenden Voraussetzungen zur Verbesserung der Versorgungsstrukturen zu schaffen. Dies belegt den Stellenwert, der in der arztinternen Diskussion den qualitätssteigernden Aspekten wie Präsenz, Kommunikation, Kooperation und Qualitätssicherung beigemessen wird. Aus ärztlicher Sicht sind vernetzte Praxen daher als Mittel zur Strukturverbesserung auch ohne Verbindung mit zugewiesenen Budgets eine wichtige Voraussetzung für die Weiterentwicklung der Versorgung. Die gesetzliche Grundlage des $\S 73$ a SGB V gibt dieser ldee auch genügend Raum, da die Zuweisung eines Budgets für ärztlich veranlaßte Leistungen lediglich als optionales Modul angelegt ist. Die wirtschaftliche Verantwortung des Hausarztes oder der vernetzten Praxis kann sich dagegen auch auf die ärztlichen Leistungen, also ohne Einbeziehung der ärztlich veranlaßten Leistungen, beschränken.

Die Vorstellungen der Krankenkassen zielen dagegen fast ausschließlich auf die Entwicklung solcher Strukturen, in denen die ärztlichen und die ärztlich veranlaßten Leistungen zu einem Gesamtbudget für die "Netzärzte" zusammengefaßt werden. Dieser Ansatz mag zwar aufgrund der bekannten Zielsetzungen der USA als dem "Mutterland" der Managed-Care-Diskussion plausibel erscheinen, er könnte jedoch die ursprüngliche ärztliche Motivation einer strukturellen Verbesserung der Versorgung und einer engeren Kooperation unter den Vertragsärzten vielleicht eher behin- 
dern als fördern. Es wird daher von großem Interesse sein zu beobachten, wie sich die einzelnen Modellansätze mit den unterschiedlichen Schwerpunkten „Versorgungskosten" und „Versorgungsqualität" auch im Hinblick auf die Akzeptanz in der Bevölkerung entwickeln werden.

\section{Mögliche Inhalte von Struktur- und Versorgungsverträgen}

Auch wenn mit dem 2. GKV-Neuordnungsgesetz die rechtlichen Grundlagen für die Entwicklung von Modellversuchen und Strukturverträgen geschaffen wurden, so darf doch nicht übersehen werden, daß die tatsächliche Implementierung derartiger Vorhaben teilweise sehr komplexe vertragliche Vereinbarungen erfordert. Dabei sind mehrere Vertragsebenen zu unterscheiden:

- Die kollektivvertragliche Ebene zwischen Krankenkasse und Kassenärztlicher Vereinigung,

- die eigentliche Ebene der Behandlung, also das Verhältnis von Versichertem und Kassenarzt,

- die Ebene der Kassenmitgliedschaft zwischen Versichertem und Krankenkasse sowie

- die Ebene der kassenärztlichen Selbstverwaltung im Verhältnis von Kassenarzt und Kassenärztlicher Vereinigung.

Der Großteil der bislang in der Öffentlichkeit diskutierten vertraglichen Angelegenheiten betraf die erste der dargestellten Ebenen, also das kollektivvertragliche Verhältnis von Krankenkassen und Kassenärztlichen Vereinigungen. Bereits hier sind die Regelungsgegenstände zum Teil außerordentlich komplex, wie in Abbildung 6 gezeigt wird. Von besonderer Bedeutung ist dabei der erste der dargestellten Punkte, nämlich die Beschreibung des Gegenstands des Versorgungsauftrags und die Definition des Versorgungsziels. Je nachdem mit welchem Anspruch dieses Versorgungsziel definiert wird, ergeben sich erhebliche Folgerungen für die Inhalte der hieraus abgeleiteten Versorgungsverträge. Folgende Elemente („Module") können Gegenstand einer Konkretisierung des ärztlichen Versorgungsauftrags sein: 


\section{Mögliche Regelungsbereiche von Strukturverträgen}

1. Gegenstand des Versorgungsauftrags (Hausärzte oder Verbund von Hausärzten und Fachärzten), Definition des Versorgungsziels;

2. Kreis der teilnehmenden Ärzte und Voraussetzungen ihrer Teilnahme;

3. Pflichten der Krankenkassen zur Vergütung, zur Mitteilung von Daten und zur Information ihrer Versicherten;

4. Pflichten der Kassenärztlichen Vereinigung;

5. Regelungen zur Vergütung im einzelnen, einschließlich der Modalitäten einer Anschubfinanzierung;

6. Regelungen zur Verteilung der Vergütung durch die Kassenärztliche Vereinigung an die Ärzte des Praxisverbundes;

7. Regelungen zur Realisierung von Wirtschaftlichkeitserfolgen bei der Versorgung, ihre Berechnung und ihre Weitergabe an die Ärzte des Praxisverbundes;

8. Regelungen zur Bestimmung von Budgets und ihrer Funktion (Orientierung oder Haftung);

9. Übermittlung von Daten zur Steuerung der Budgets;

10. Folgen für die Gesamtvergütung, die Wirtschaftlichkeit der Versorgung im Hinblick auf Wirtschaftlichkeitsprüfungen sowie die Berücksichtigung der sich aus dem Fremdkassenausgleich ergebenden Folgerungen.

1. Koordinationspflichten und/oder - bei integrierten Systemen Pflichten zur Zusammenarbeit mit den die Versorgung ergänzenden Einrichtungen (vgl. auch Nr. 10).

2. Interne Regelung der Präsenzpflichten.

3. Errichtung einer Leitstelle (Beschreibung der Funktion der Leitstelle, personelle Besetzung etc.).

4. Qualitätssicherungspflichten (z. B. Teilnahme an Qualitätszirkeln, Einholung von Zweitmeinungen).

5. Dokumentationspflichten im Hinblick auf besondere Kooperationsverfahren.

6. Pflichten zur Befundübermittlung mit Einwilligung des Patienten an die mitwirkenden Ärzte. 
7. Finanzierungspflichten für den gemeinschaftlichen Kooperationsaufwand (z. B. Leitstelle).

8. Errichtung eines EDV-Systems zur besseren Informationsverarbeitung innerhalb der Gemeinschaft (z. B. "Abruf"Regelungen unter Berücksichtigung der ärztlichen Schweigepflicht, Zentralisierung der Dokumentation im Sinne der hausärztlichen Dokumentation).

9. Innergesellschaftliche Regelungen zur Einhaltung des Budgets (nur in Strukturverträgen mit Budgetverantwortung).

10. Optionale Regelungen im Fall der Erweiterung des Versorgungsauftrags auf komplexe Versorgungsformen (z. B. Übernahme der Koordination einer komplexen Versorgung - wie etwa Rehabilitation oder geriatrische Versorgung - im Zusammenwirken mit anderen Behandlern, Kooperationsverträge mit Krankenhäusern, Zusammenarbeit mit Krankenkassen).

Eine noch weitergehende Stufe der Komplexität vertraglicher Regelungen öffnet sich, wenn im Sinne kombinierter Budgets veranlaßte Leistungen in die Budgetverantwortung der am Modellvorhaben oder an Strukturverträgen teilnehmenden Kassenärzte mit einbezogen werden. Die Abstimmung der sich hieraus ergebenden komplexen Regelungen mit dem bisherigen, ohnehin bereits komplexen System der Errechnung und Verteilung kassenärztlicher Gesamtvergütungen ist überhaupt nur möglich, wenn - in der Hoffnung auf Akzeptanz bei sozialgerichtlicher Überprüfung eine Reihe von Fragen vereinfachten Lösungen zugeführt werden.

In Abbildung 7 ist hinsichtlich des Teilaspekts der Berechnung eines kombinierten Budgets ein Ablaufplan wiedergegeben, wie er im Rahmen des BKK-Modellvorhabens in Berlin angewendet werden soll. Die Berechnung von kombinierten Budgets verursacht im übrigen gerade bei den Betriebskrankenkassen erhebliche Probleme, da aufgrund der großen Zahl der Betriebskrankenkassen und der Verteilung der Versicherten einzelner großer Betriebskrankenkassen auf die Bereiche sehr vieler Kassenärztlicher Vereinigungen bereits mit einem einzigen Modellversuch in einer einzigen Kassenärztlichen Vereinigung indirekt sehr viele Kassenärztliche Vereinigungen über den sogenannten „Fremdkassenausgleich" vertragsrechtlich berührt werden. Dies 
ist auch der Grund, warum gerade der Bundesverband der Betriebskrankenkassen eine bundesweite vertragliche Regelung mit der Kassenärztlichen Bundesvereinigung zu dieser Problematik anstrebt. 


\section{Berechnung des kombinierten Budgets}

Ambulante Versorgung

Gesamtvergütung

Anzahl Versicherte der BKKen

Durchschnittliche Ausgaben

für die ambulante Versorgung pro Versicherten
Arzneimittelversorgung, stationäre Versorgung,

Rehabilitation

Vorjahresausgaben

Anzahl Versicherte der BKKen

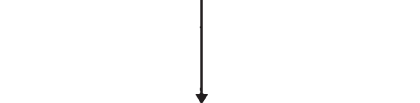

Durchschnittliche Ausgaben für veranlaßte Leistungen pro Versicherten<smiles>CI</smiles>

Alters- und geschlechtsspezifische Gewichtung auf der Grundlage des Risikostrukturausgleichs

Alters- u. geschlechtsspezifische Kopfpauschlalen

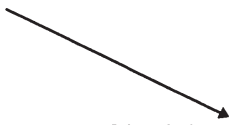

Multipliziert mit der jeweiligen

Anzahl der eingeschriebenen

Versicherten pro Altersklasse

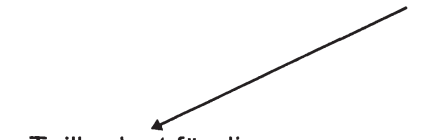

Teilbudget für die ambulante Versorgung

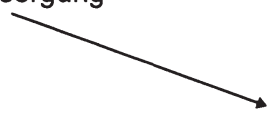

Kombinierte Budgets

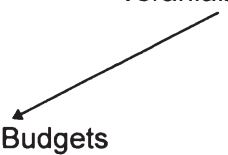

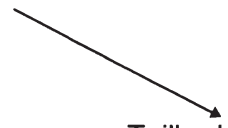

Teilbudget für veranlaßte Leistungen 


\section{Bonusverträge auf kollektiver und individueller Ebene}

Bereits im Gesetzgebungsverfahren zum 2. GKV-Neuordnungsgesetz wurde von seiten der pharmazeutischen Industrie und der Apothekerverbände kritisiert, daß die Einführung von Modellvorhaben und Strukturverträgen letztendlich auf die Beteiligung von Kassenärzten an Einsparungen im Bereich veranlaßter Leistungen abzielte. Dies könne nicht hingenommen werden, da der Kassenarzt auf diese Weise von einem "Herunterfahren" der Versorgung direkt profitiere.

Diese stark vereinfachende Darstellung von seiten der pharmazeutischen Industrie und der Apothekerverbände übersieht, daß "Bonusregelungen" in der kassenärztlichen Versorgung seit jeher allgegenwärtig sind (vgl. Abbildung 8 ). So ist den Kassenärzten bereits seit den 20er Jahren der "Bonus" bekannt, der ihnen dann zuteil wird, wenn in den Verhandlungen mit den Krankenkassen ein Wachstum der Gesamtvergütung mit dem Hinweis darauf durchgesetzt werden kann, daß in anderen Ausgabensektoren, insbesondere in den durch Kassenärzte veranlaßten Leistungsbereichen, die Ausgaben in Grenzen gehalten werden konnten. Den Kassenärzten ist ebenfalls bereits seit Jahrzehnten bekannt, daß sie ihr individuelles kassenärztliches Honorar dadurch sichern können, daß sie durch eine Begrenzung der veranlaßten Arzneimittelausgaben einen Regreß in der Wirtschaftlichkeitsprüfung nach Durchschnittswerten vermeiden.

Abb. 8

\section{Bonusregelungen in der kassenärztlichen Versorgung}

\section{auch vor 1993}

1. Kollektiv-Bonus durch Wachstum der Gesamtvergütung bei Verhandlungsspielraum durch niedrige Arzneimittelausgaben

2. Individual-Bonus durch Vermeidung von Regressen in der Wirtschaftlichkeitsprüfung nach Durchschnittswerten

ab 1993

3. Kollektiv-Bonus durch Vermeidung von Überschreitungen der kollektiven Arzneimittelbudgets

4. Bonus durch Vermeidung von Richtgrößen-Regressen ab 1998

5. Kollektiv-Bonus durch "kombinierte Budgets" auf KV-Ebene

6. Individual-Bonus durch „kombinierte Budgets“ in Modellversuchen und Strukturverträgen 
Mit dem Gesundheitsstrukturgesetz kamen ab dem Jahr 1993 zwei weitere "Bonusaspekte" hinzu. Zum einen geschah dies in Form des Kollektivbonus durch gemeinsame Anstrengungen zur Vermeidung von Gesamtvergütungskürzungen aufgrund von Überschreitungen der kollektiven Arznei- und Heilmittelbudgets, zum anderen wurde bereits im Jahr 1993 die rechtliche Hülse einer budgetablösenden Richtgröße eingeführt, die den Arzt motivieren sollte, durch eine "wirtschaftliche" Arzneimittelverordnung den Individualbonus zur Vermeidung eines Richtgrößenregresses anzustreben.

Es ist unklar, warum die vier genannten "historischen" Bonusregelungen von der pharmazeutischen Industrie und den Apothekerverbänden faktisch akzeptiert wurden, während die ab dem Jahr 1998 möglichen Bonusregelungen nunmehr den Arzt angeblich zu unärztlichem Handeln motivieren sollen. Bei einer solchen Argumentation wird übersehen, daß die Vermeidung eines Malus stets auch einen Bonus darstellt, da daraus in beiden Fällen durch eine bestimmte gewünschte Verhaltensweise zur "Belohnung" und Verstärkung dieser Verhaltensweise ein höheres Honorar resultiert.

Dabei ist der Bonusgedanke neuerer Prägung deswegen für die Ärzte viel eher akzeptabel, weil auf diese Weise endlich zum Ausdruck kommt, daß die ärztliche Vergütung nicht einfach Dispositionsmasse der Krankenkassen zur Finanzierung anderer Leistungsbereiche ist, sondern vielmehr von den Kassenärzten durch Inkaufnahme zunehmend höherer beruflicher Belastung hart erarbeitet wird. Der Bonusgedanke neuerer Prägung hebt damit lediglich die Herabwürdigung ärztlicher Leistung auf, ohne etwa die Motivation für Einsparungen in irgendeiner Weise "unethischer" zu machen, als dies bereits den vier "historischen" Bonusregelungen unterstellt werden konnte.

Wenn pharmazeutische Industrie und Apothekerverbände, die von sämtlichen direkten Malusregelungen in der Vergangenheit trotz ihrer erheblichen Mitverantwortung für die Entwicklung der Arzneimittelausgaben verschont worden sind, nur diejenigen Modelle gelten lassen wollen, in denen die Vergütung der ärztlichen Leistungen zur Dispositionsmasse der Krankenkassen herabgewürdigt wird, dann können sie für die Kassenärzte keine ernstzunehmenden Gesprächspartner mehr sein. Wer nicht erkennt, daß die Erzielung von Ausgabeneinsparungen durch Effizienzsteige- 
rung in der Arzneimittelverordnung einen erheblichen persönlichen Einsatz jedes einzelnen Arztes erfordert und damit auch eine entsprechende Vergütung rechtfertigt, hat offensichtlich keine Kenntnis vom tatsächlichen Verordnungsgeschehen und damit auch die Möglichkeit verspielt, in dieser Diskussion ernstgenommen zu werden.

In diesem Zusammenhang sollte nicht unerwähnt bleiben, daß die in Abbildung 8 als Nr. 5 genannte Möglichkeit des Kollektivbonus durch "kombinierte Budgets" auf KV-Ebene nicht etwa mit dem 2. GKV-Neuordnungsgesetz neu geschaffen wurde, sondern nach Auffassung des Bundesministeriums für Gesundheit aufgrund der Regelungsnorm des $\S 83$ [1] SGB V bereits seit langem gegeben ist. Auf diese Weise ist es den Kassenärztlichen Vereinigungen und den Krankenkassen gestattet, kombinierte Budgets z. B. für ärztliche Behandlung und Arzneimittel nicht nur auf der individuellen, sondern auch auf der kollektiven Ebene zu vereinbaren. Dies habe, so die Interpretation des Bundesministeriums für Gesundheit, bereits für den gesamten Zeitraum der gesetzlichen Kollektivbudgets gegolten, so daß es bereits vor 1997 möglich gewesen ist, etwa Budgetunterschreitungen anteilig zur Verbesserung der kassenärztlichen Gesamtvergütung zu verwenden. Somit sind künftig bei den vertraglichen Möglichkeiten zur Weiterentwicklung der Versorgungsstrukturen in Richtung auf kombinierte Budgets eine "kollektive Ebene" und eine "individuelle Ebene" zu unterscheiden (vgl. Abbildung 9).

Abb. 9

\section{Vertragliche Möglichkeiten zur Weiterentwicklung der Versorgungsstrukturen}

1. "Kollektive Ebene" (§ 83 [1] SGB V)

- z. B. Gesamtbudget für ärztliche Behandlung, Arzneimittel und Heilmittel

2. "individuelle Ebene"

- Strukturverträge für Hausärzte und vernetzte Praxen (§73 a SGB V)

- Modellvorhaben zu Organisations- und Vergütungsformen ( $\$ 63 \mathrm{ff}$. SGB V) 


\section{Wie realistisch sind Einsparungen?}

Unabhängig von den neugeschaffenen rechtlichen Optionen zur Einbeziehung von Einsparungen, insbesondere bei den Arzneimittelausgaben, in die Verhandlungen zur Weiterentwicklung der kassenärztlichen Gesamtvergütung muß die Frage gestellt werden, inwieweit solche Einsparungen angesichts der demographischen Herausforderungen und der medizinischen Entwicklungen gerade auf dem Arzneimittelsektor überhaupt realistisch sind. In den vergangenen zwanzig Jahren haben die Arzneimittelausgaben - jedenfalls in den "Nicht-Reformjahren" - jährlich um mehr als $6 \%$ zugenommen. Selbst bei strengster Indikationsstellung und unter faktischem Ausschluß der sogenannten „umstrittenen“ Arzneimittel ergibt sich hieraus ein offensichtlich nicht aufhaltbares "natürliches" Wachstum der Arzneimittelausgaben in der GröBenordnung von jährlich mindestens $3 \%$.

Aus dieser Feststellung folgt unmittelbar die Frage, was unter diesen Voraussetzungen für die Krankenkassen "Einsparungen“ sind (vgl. Abbildung 10). Werden es die Krankenkassen als Einsparungen anerkennen, wenn die Kassenärzte unter Inkaufnahme von Patientenprotesten auf sog. „umstrittene" Arzneimittel, deren Verordnung ja keineswegs ausgeschlossen ist, zunehmend verzichten und damit das Ausgabenwachstum auf $3 \%$ pro Jahr reduzieren können? Ist eine solche Minderung überhaupt realistisch, wenn die Verlagerung ehemals stationär erfolgter Behandlungen in die ambulante Versorgung im bisherigen Umfang anhält? Verlangen die Krankenkassen etwa gar ein "Nullwachstum“, bevor sie die Kassenärzte für ihre mit erheblicher Mehrarbeit verbundenen Anstrengungen vergüten? Oder werden die Krankenkassen angesichts faktisch stagnierender Entwicklungen der Beitragseinnahmen sogar fordern, daß erst bei nachgewiesenen rückläufigen Arzneimittelausgaben eine Anhebung der ärztlichen Gesamtvergütungen in Frage kommt? 


\section{Voraussichtliche Entwicklung der Arzneimittelausgaben und Einsparmöglichkeiten $(1997=100)$}

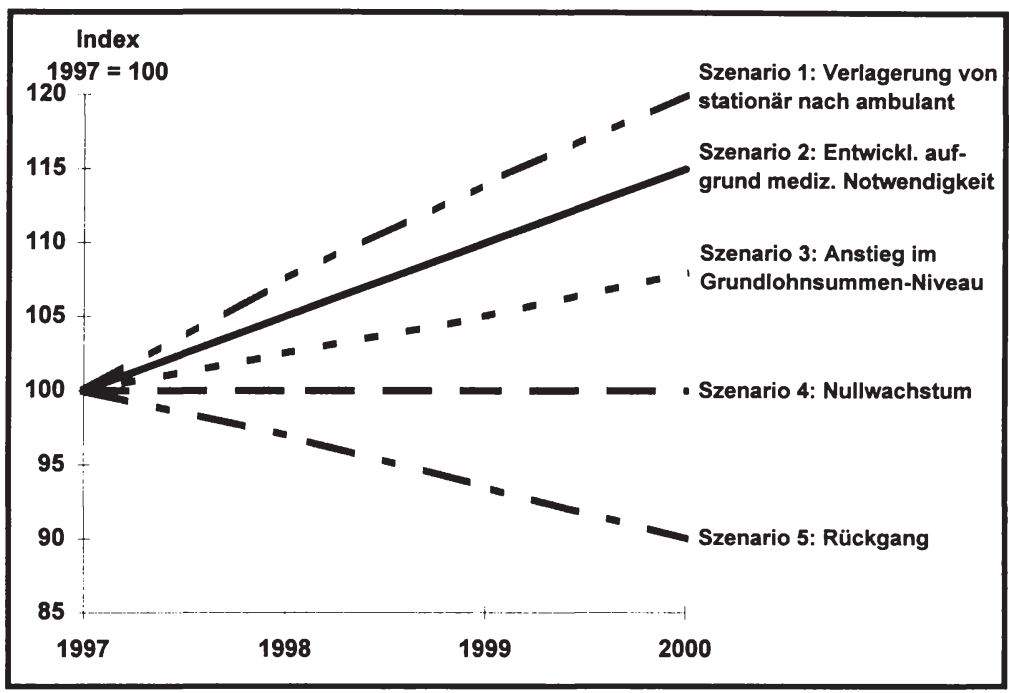

Nach den bisherigen Erfahrungen aus den Verhandlungen mit den Krankenkassen fordern diese tatsächlich, daß die Arzneimittelausgaben unter das jeweilige Vorjahresniveau zurückzugehen haben, bevor auf kollektiver oder individueller Ebene eine Beteiligung der Kassenärzte an den Einsparfolgen in Frage kommen soll. Man braucht kein Prophet zu sein, um vorauszusagen, daß bei einer dermaßen kurzsichtigen Haltung der Krankenkassen Verträge zu kombinierten Budgets bereits tot sind, bevor sie das Licht der Welt erblickt haben. Ein Versorgungsmanagement, dessen Zielsetzung sich darin erschöpft, die einzelnen Sektoren der Versorgung jeweils unter Vorjahresniveau zu drücken, verdient nicht die Bezeichnung "Management". Wir wissen heute, daß eine gezielte Stärkung der Arzneimittelbehandlung bei verschiedenen Krankheitsbildern zwar einen Anstieg der Arzneimittelausgaben bedingt, jedoch aufgrund von Einsparungen, insbesondere im stationären Bereich, letztlich sowohl zu einer humaneren Krankenbehandlung als auch zu einem effizienteren Ressourcenverbrauch beiträgt. Solange diese Zusammenhänge nicht beachtet 
werden, dürften Strukturverträge mit kombinierten Budgets mittelund langfristig keine Chance auf Verwirklichung haben.

\section{Schlußbemerkung}

Mit dem 2. GKV-Neuordnungsgesetz wurden Elemente in die gesetzliche Krankenversicherung aufgenommen, mit denen eine Übertragung des Managed-Care-Gedankens US-amerikanischen Vorbildes auf die solidarische Krankenversicherung deutscher Prägung ermöglicht werden sollte. Neben der Aufstockung der finanziellen Basis der gesetzlichen Krankenversicherung und der Straffung des Leistungskataloges bietet nur noch diese strukturelle Weiterentwicklung eine Option, mit der das zunehmende Auseinanderdriften von Leistungsbedarf und Finanzierung gestoppt werden kann. Allerdings gibt es gerade im internationalen Vergleich eine Reihe von Hindernissen, die selbst der Einführung sinnvoller Elemente von Managed Care in das deutsche Gesundheitswesen entgegenstehen.

Abb. 11

Mögliche Hindernisse für die Einführung sinnvoller Elemente von Managed Care in das deutsche Gesundheitswesen

1. geringere Rationalisierungsreserven in Deutschland

2. überhöhtes Anspruchsniveau der Versicherten

3. Vorrang des Datenschutzes vor Gesundheitszielen

4. kartellrechtliche Behinderung von Therapie-Empfehlungen

5. Versicherungsmonopol einer gesetzlichen Zwangsversicherung

6. kostentreibender "solidarischer Wettbewerb"

7. offentlich-rechtlicher Zulassungsstatus der Kassenärzte

8. Sicherstellungsauftrag der Kassenärztlichen Vereinigungen

In der hierauf bezogenen Abbildung 11 ist auch der Sicherstellungsauftrag der Kassenärztlichen Vereinigungen als mögliches Hindernis für eine sinnvolle strukturelle Weiterentwicklung aufgeführt. Dies gilt jedoch nur für den Fall, daß die Kassenärztlichen Vereinigungen ein überholtes Verständnis des Sicherstellungsauftrages bewahren und sich nicht den kommenden Herausforderungen stellen würden. Angesichts der erheblichen Dynamik und der "Aufbaustimmung" an der ärztlichen Basis dürfte diese Gefahr jedoch eher gering sein. Problematischer ist dagegen schon das Versicherungsmonopol einer gesetzlichen 
Zwangsversicherung und insbesondere der zwischen den Trägern dieses Monopols inszenierte "solidarische Wettbewerb". Durch diese unglückliche Konstellation droht die Seite der Versicherungsträger die Entwicklung zukunftsweisender Versorgungsmodelle dem Ringen um die eigene Existenz unterzuordnen. Die gesetzliche Krankenversicherung ist jedoch kein Selbstzweck. Daher sollte der Gesetzgeber möglichst umgehend dazu beitragen, daß die Parameter des Kassenwettbewerbs endlich auf eine vernünftige Versorgung der Versicherten durch sinnvolle Entwicklung der Versorgungsstrukturen fokussiert werden. Erst dann werden die mit dem 2. GKV-Neuordnungsgesetz geschaffenen Möglichkeiten ihre gewünschten Wirkungen entfalten können. 


\section{Chancen und Risiken von Strukturverträgen und Modellvorhaben}

Wolfgang Schmeinck

Von meinen Vorrednern ist das, was zur Lage der GKV im allgemeinen und was zur Situation und zur Perspektive von Strukturverträgen und Modellversuchen im besonderen $\mathrm{zu}$ sagen ist, weitestgehend vorgetragen worden. Ich habe nichts vernommen, dem ich nicht zumindest im großen und ganzen zustimmen könnte. Angesichts der Tatsache, daß meine Redezeit schon vor ungefähr 50 Minuten zu Ende gegangen ist, dient es möglicherweise dem Tagungsmanagement, wenn ich mich auf einige wenige Bemerkungen beschränke.

\section{Derzeit ist keine nennenswerte Alternative zu Managed-Care-Verfahren in Sicht}

Es ist auch heute aus den bisherigen Diskussionsbeiträgen deutlich geworden, daß die Erwartungen, die im Hinblick auf Qualität, Quantität und Finanzierbarkeit der medizinischen Versorgung an die gesetzliche Krankenversicherung bestehen, mit konventionellen Methoden allein kaum noch werden bedient werden können. Versorgungsqualität und Leistungsumfang sind möglicherweise in den vergangenen Jahren nicht so sehr als Zielkonflikt zur Kenntnis genommen worden, wie dies heute geschieht, da wir erhebliche Engpässe auf der Einnahmenseite der gesetzlichen Krankenversicherung registrieren und der Tatsache ins Auge sehen müssen, daß sich dies auf absehbare Zeit kaum nachhaltig ändern wird. Wollte man bestehende Einnahmen- und Ausgabenstrukturen mehr oder weniger nur fortschreiben, wie dies das Verhaltensmuster der vergangenen Jahre und Jahrzehnte war, ginge dies an den Notwendigkeiten vorbei. Ohne zumindest eine sehr intensive Diskussion über eine Erweiterung der Finanzierungsbasis der GKV auf der einen und neue Versorgungsformen auf der anderen Seite ist der angedeutete Zielkonflikt mit Sicherheit nicht zu lösen. Neue Versorgungsformen konkretisieren sich nicht nur, aber im wesentlichen in Modellvorhaben nach $\S 63 \mathrm{ff}$. SGB V und Strukturverträgen nach § 73 a SGB V.

Ziel solch neuer Versorgungsformen ist zweifellos die Konzentration auf das medizinisch Notwendige und Sinnvolle, wie schwierig 
seine Bestimmung im einzelnen auch immer sein mag. Als Entscheider über und Veranlasser von Leistungen sowie erste Anlaufstelle der Patienten steht der Arzt weiterhin im Mittelpunkt auch der neuen Versorgungsformen. Die übrigen Leistungserbringer im Gesundheitswesen nehmen die Rolle eines „Verordnungsdienstleisters" ein.

Die neuen Versorgungsformen sollen vor allem

- die organisatorisch wie finanziell strikte Trennung zwischen der stationären und der ambulanten Versorgung aufheben,

- die häufig mangelnde Kooperation vor allem in Einzelpraxen tätiger Ärzte untereinander verbessern,

- eine Qualitätssicherungsroutine bei den eigenen ärztlichen wie auch den veranlaßten und verordneten Leistungen erzeugen.

Diese Ziele mögen trivial erscheinen. Es ist auch überhaupt nicht ausgeschlossen, daß sie auch in konventionellen Strukturen des deutschen Gesundheitswesens ganz oder teilweise erreichbar sind. Tatsache ist allerdings, daß einer immerhin doch langjährigen Diskussion über Versorgungsqualität und rationale Medizin bislang wenig benennbare Ergebnisse gefolgt sind. Vor diesem Hintergrund sehe ich kurz- bis mittelfristig weder in der "konventionellen" Verhandlungslandschaft noch in der wissenschaftlich/politischen Diskussion eine ebenbürtige Alternative zu Modellversuchen und Strukturverträgen.

\section{Wettbewerb oder Einheitslösung?}

Gerade auch angesichts der Unbeholfenheit, mit der sich die gesetzliche Krankenversicherung auf dem einen oder anderen für sie neuen Wettbewerbsfeld bewegt, betone ich: Wettbewerb ist ein Suchprozeß nach besseren Lösungen. Bewähren sie sich, können sie imitiert werden, bewähren sie sich nicht, werden sie angepaßt oder verworfen. Die kreative Chance liegt zumindest bis auf weiteres im Nebeneinander unterschiedlicher Lösungen.

Vor diesem Hintergrund war der Gesetzgeber gut beraten, als er bei Modellvorhaben ausdrücklich zugelassen hat, daß nicht nur die Verbände der Krankenkassen, sondern auch einzelne Kran- 
kenkassen unmittelbar die notwendigen Verträge für Modellversuche schließen können.

Einheitslösungen unterliegen einer solchen innovationsfördernden Dynamik nicht.

An dieser Stelle teilen sich ganz offensichtlich die strategischen Interessen von Kassen- und KV-Seite. Ob letztere bei einer Vielzahl unterschiedlicher Modellversuche perspektivisch ihre Monopolfunktion gefährdet sehen muß und/oder ob sie - was ich gar nicht grundsätzlich bestreiten will - praktische Umsetzungsprobleme bei einem Nebeneinander vieler Modellversuche zu fürchten hat, kann dahinstehen. Wenn die Kassenärztliche Bundesvereinigung jedenfalls vorschlägt, rahmenvertragliche Regelungen über vernetzte Praxen und kombinierte Budgets unterhalb der Schwelle des Bundesmantelvertrages einheitlich und gemeinsam zu treffen, würde damit die wettbewerbliche und kreative Wirkung von Strukturverträgen auf jeden Fall erheblich reduziert. Dies halte ich in der gegenwärtigen Entwicklungs- und Umsetzungsphase der neuen Instrumente für kontraproduktiv, räume aber ein, daß dieses Thema in meiner eigenen Kassenart nicht einheitlich gesehen wird; ich schließe nicht aus, daß einzelne Kassen und Kassenarten auf den Vorschlag von Strukturverträgen als einheitliche und gemeinsame und insoweit vielleicht auch bequeme und auf den ersten Blick komfortable Lösung eingehen werden. Ob die Ärzteschaft glaubwürdig ist, wenn sie einerseits die freie Arztwahl propagiert und andererseits eine Beschränkung von wettbewerblichen Angeboten neuer Versorgungsformen organisiert, darf bezweifelt werden.

Vermutlich bedarf es noch eines regelrechten Marketingfeldzugs in der Ärzteschaft für wettbewerbliche Alternativen, weil bislang vermutlich dort die Irritationen größer sind und die Risiken neuer Versorgungsformen höher eingeschätzt werden als die systematischen Chancen. Um so dringlicher ist es, Qualitätsverbesserungen und Effizienzsteigerungen durch praktische Ergebnisse, insbesondere von Modellvorhaben, erfahrbar zu machen.

\section{Modellvorhaben vor Strukturverträgen}

Strukturverträge sind die experimentellere Variante der hier in Rede stehenden Instrumente; das gilt sowohl für die Strukturmodelle nach $\S 63$ [1] als auch für die Leistungsmodelle nach $\S 63$ [2] SGB V. Der Gesetzgeber hat ihren experimentellen Charakter 
u. a. dadurch unterstrichen, daß er im Vergleich zu Strukturverträgen die Eingriffmöglichkeiten in bestehende rechtliche Bedingungen umfassender gestaltet hat, dafür auf der anderen Seite aber eine zeitliche Befristung und eine wissenschaftliche Evaluierung vorsieht.

Meines Erachtens hätte das Prinzip der Freiwilligkeit für die Teilnahme der Versicherten es verdient, ähnlich wie für die Strukturverträge auch für Modellversuche gesetzlich verankert zu werden. Der Gesetzgeber hat statt dessen die Regelung dieser Frage auch dies mag den experimentellen Charakter unterstreichen der Satzung der jeweiligen Kasse überlassen. Beim Berliner Modellversuch der Betriebskrankenkassen ist jedenfalls über eine Satzungsregelung die strikte Freiwilligkeit der Teilnahme der Versicherten sichergestellt.

Vor diesem Hintergrund halte ich es für naheliegend, fast für zwingend, daß neue Versorgungsstrukturen und ggf. auch neue Leistungsinhalte zunächst in befristeten Modellversuchen getestet werden und nur dann, wenn sie sich dabei bewähren, in unbefristete Strukturverträge überführt werden. Ich nehme zur Kenntnis, daß dies teilweise anders gesehen und auch anders organisiert wird.

\section{Modellversuch „Berliner Praxisnetz“}

Im Berliner Praxisnetz, einem Strukturmodell nach § 63 [1] SGB $\mathrm{V}$, arbeiten die Betriebskrankenkassen und die Techniker-Krankenkasse gleichberechtigt mit der Kassenärztlichen Vereinigung Berlin zusammen. Die Anlaufprobleme waren nicht unerheblich. Es bedarf qualifizierter Anstrengung, Versicherte zum freiwilligen (und auch kurzfristig wieder kündbaren) Beitritt in ein solches Netz zu bewegen. Wir haben wenig Interesse daran, diesem Netz nur Junge und Gesunde zuzuführen. Vielmehr benötigen wir gerade auch kranke Netzteilnehmer, weil nur durch die Beobachtung ihres Behandlungs- und Versorgungsverlaufs geprüft werden kann, ob Qualität und Rationalität der Versorgung in der abgesprochenen Weise organisiert und verbessert werden.

Von ärztlicher Seite sind niedergelassene fach- und hausärztlich tätige Ärzte aus Berlin Netzteilnehmer, die sich an einer Ausschreibung der Kassenärztlichen Vereinigung Berlin beteiligt haben. Selbstverständlich ist auch für die Ärzte die Teilnahme frei- 
willig. Voraussetzung ist allerdings neben einem Mindestanteil an BKK- und TK-Patienten eine kommunikationsfähige Praxis-EDV. Derzeit arbeiten etwa 270 Hausärzte, Internisten, Kinderärzte, Urologen, Gynäkologen usw. in diesem Projekt eng zusammen.

Es wurden bereits Qualitätszirkel eingerichtet, in denen gemeinsame Behandlungsleitlinien erarbeitet werden. Das Praxisnetz ist in Unternetze aufgeteilt, in denen jeweils etwa 20-40 Ärzte ein Team bilden, die in einem Bezirk oder in benachbarten Bezirken arbeiten. In diesen Teams findet der wichtigste Teil der Qualitätssicherung statt, wird die Vernetzung weiter vorangetrieben, werden Probleme und Verbesserungsmöglichkeiten der Netzarbeit besprochen und Absprachen über Diagnose und Therapieleitlinien getroffen. Neben medizinisch-inhaltlichen Fragen geht es dabei in der jetzigen Phase vor allem auch um die Entwicklung notwendiger Managementverfahren und -instrumente.

Die Kompetenz in medizinischen Fragen bleibt allein Angelegenheit der beteiligten Ärzte. Dies gilt sowohl für die tägliche Arbeit im Praxisnetz als auch für die Diskussion in den Qualitätszirkeln und die Koordinierung in einem medizinischen Beirat.

Unterstützt werden die Ärzte durch eine Leitstelle. Von ihr erhalten sie Informationen über Heilmittelerbringer, ambulante Vorsorge-, Rehabilitations- und Pflegeeinrichtungen, Krankenhäuser und ambulante Operationszentren; auch bei der Bereitstellung von Hilfsmitteln ist die Leitstelle behilflich.

Während eine Vernetzung Gegenstand nahezu aller neuer Versorgungsformen ist, hat das Berliner Modell mit einem kombinierten Budget eine weitere, durchaus besondere Ausrichtung:

Die teilnehmenden Ärzte übernehmen die Verantwortung für die gesundheitliche Versorgung der Versicherten auch in finanzieller Hinsicht. Diese Verantwortung erstreckt sich nicht mehr nur auf die eigene ärztliche Tätigkeit, sondern auch auf andere Leistungsbereiche wie Krankenhausbehandlung, Arzneimittelversorgung usw.

Das kombinierte Budget kann zu einem höheren Ertrag der Netzärzte führen, wenn sie das errechnete Budget auch tatsächlich unterschreiten. Die erzielten Einsparungen werden zu einem Drittel den Netzärzten zugewiesen; ein weiteres Drittel wird zum 
weiteren Ausbau des Netzes verwendet, und das letzte Drittel verbleibt bei den beteiligten Kassen und damit den am Netz teilnehmenden Versicherten.

Da insbesondere in der Anlaufphase das Budget vergleichsweise gering sein wird, werden Einzelfälle mit besonders hohen Ausgaben, die der Höhe nach versicherungsmathematisch nicht von der Anzahl der teilnehmenden Versicherten getragen werden können und das kombinierte Budget sprengen würden, auf der Ausgabenseite nicht eingerechnet. Damit bleibt ein kritischer Teil des Morbiditätsrisikos bei den beteiligten Kassen - wohin es auch grundsätzlich gehört. Die Behandlung von besonders kostenaufwendigen Fällen erfolgt in aller Regel im stationären Bereich; die Netzärzte sollen jedoch vor allem den Teil des Morbiditätsrisikos übernehmen, der im wesentlichen auch in ihrem eigenen und unmittelbaren Tätigkeits- und Verantwortungsbereich liegt.

Das kombinierte Budget und seine ökonomischen Anreize sind nach bisheriger Erfahrung eine wichtige Voraussetzung für das Gelingen des Projekts. Finanzielle Anreize sind gegenüber den Netzteilnehmern wohl auch frühzeitiger und einfacher kommunizierbar als eine Verbesserung der Behandlungs- und Versorgungsqualität.

Um einem Mißverständnis vorzubeugen: Die am Netz teilnehmenden Versicherten werden für ihre Teilnahme am Praxisnetz belohnt und nicht etwa dafür, daß sie keine Leistungen in Anspruch nehmen, wie dies in anderen Modellen vorgesehen ist. Eine Beitragsrückerstattung für Versicherte, die auf Arztbesuche verzichten und auf diese Weise keine Ausgaben verursachen, hat sich bereits in wissenschaftlich begleiteten Modellversuchen als wenig taugliches Mittel erwiesen. Außerdem geht es im Berliner Praxisnetz gerade nicht darum, die medizinische Versorgung einzuschränken, sondern darum, sie qualitativ zu verbessern und wirtschaftlicher zu gestalten.

$\mathrm{Da}$ am Netz teilnehmende Versicherte langfristig am qualitativen wie am ökonomischen Erfolg des Netzes beteiligt werden, besteht nicht nur ein Anreiz für die Einschreibung in das Netz, sondern auch für ihre aktive Mitwirkung an Qualitätsverbesserungen und Einsparungen. 
Die Akzeptanz des Berliner Netzes nimmt zu. Dies geschieht allerdings langsamer, als es sich ungeduldige Gemüter zuvor ausgerechnet haben. Auch dies ist eine Erfahrung der praktischen Arbeit.

\section{Fazit}

Modellvorhaben und Strukturverträge sind das Experimentierfeld schlechthin für Formen der integrierten Versorgung. Würde es nicht genutzt, ginge dies perspektivisch auch zu Lasten der Leistungsfähigkeit und damit der Akzeptanz der gesetzlichen Krankenversicherung. Für wichtiger in der derzeitigen Entwicklungsphase halte ich Modellversuche - gerade auch wegen der Chan$\mathrm{ce}$, mit ihrer Hilfe Defizite im Bereich des Managements von Behandlungs- und Versorgungsabläufen abzubauen.

Die inzwischen verschiedentlich bereits geschmähte wettbewerbliche Vielfalt neuer Versorgungsformen ist $m$. E. durchaus akzeptabel. Allerdings darf Wettbewerb nicht zum Selbstzweck werden, dürfen sich seine Ergebnisse nicht von gemeinsamen Versorgungszielen des Gesundheitswesens entfernen. Notwendige, wenn auch für sich allein nicht hinreichende Bedingung dafür ist, daß Modellversuche und Strukturverträge nicht vorrangig von Marketinggesichtspunkten einzelner Kassen oder Kassenarten bestimmt werden. Die Bereitschaft, kassenartenübergreifend neue Versorgungsformen zu erproben, ist ein gutes Korrektiv gegen solche Ambitionen.

Ein Copyright für erfolgreiche Modelle gibt es ohnehin nicht. 


\section{Der Leistungskatalog der Krankenkassen im Lichte des Solidaritätsprinzips}

Alexander P. F. Ehlers

\section{Einleitung}

Die gesetzliche Krankenversicherung (GKV) mit ihrem Leistungskatalog war im Ergebnis der Sozialgesetzgebung eine der Notwendigkeiten, die Bismarck Ende des letzten Jahrhunderts auf den Weg gebracht hat. Ein wesentlicher Kernpunkt der Krankenversicherung war neben dem Subsidiaritätsprinzip und dem Prinzip der Eigenverantwortung das Solidaritätsprinzip. Es war unbestritten, daß eine staatliche Initiative zur Absicherung der Arbeiter gegen das Kosten- und Einkommensrisiko im Sinne einer „Vorleistung" als ursprünglich gedachte Hilfe zur Selbsthilfe angemessen und sachgerecht war.

Heute baut die GKV auf dem Sozialstaatprinzip auf. Das Grundgesetz hat die Sozialstaatlichkeit zwar ausdrücklich in die Verfassung aufgenommen und zu einem eigenständigen leitenden Verfassungsprinzip erhoben (Art. 20 I GG: sozialer Bundesstaat; Art. 28 I GG: sozialer Rechtsstaat)14. Jedoch ist dieses Staatsziel, geprägt durch "Weite und Unbestimmtheit"15, nicht durch die Verfassung selbst definiert, so daß wegen seiner Offenheit weder Verwaltung noch Rechtsprechung aus diesen Anweisungen Konkretisierung und Umsetzung in einfaches Recht entnehmen können. Schon deshalb ist der Begriff der "Sozialstaatlichkeit" ausfüllungsbedürftig. Die Verwirklichung obliegt dabei nahezu vollständig dem Gesetzgeber16 mit der Folge, daß diese auch einer richterlichen Rechtsfortbildung verschlossen bleibt. Somit spiegelt sich das Sozialstaatprinzip in drei Erscheinungsformen wider: in „sozialer Gerechtigkeit", gleichmäßiger Förderung aller17, „sozialem Ausgleich", der insbesondere im Fall der Aufopferung geboten ist, und in „sozialer Sicherung“. Diese soll vor allem den Schutz der sozialen Existenz gegen die "Wechselfälle des Le-

14 Degenhardt, Staatsrecht I, § 4 Rn. 354; Merten, in: Schulin, Handbuch des Sozialversicherungsrechts, Bd. 1 (Krankenversicherungsrecht), § 5 Rn. 20.

15 BVerfGE 65, 182 ff. (193); BVerfG SozR 5870, § 10 Nr. 1.

16 Papier, in: SRH 3 Rn. 6; BVerfG SozR 5870, § 10 Nr. 1.

17 BVerfGE 5, 85 ff. (198). 
bens" gewährleisten 18. Den Ausgleich innerhalb der Gemeinschaft stellt die Beitragsbemessung nach dem Einkommen und nicht nach dem Versichertenrisiko sicher.

Bei der Installation der GKV ging es zunächst um die Sicherung des Lebensunterhaltes durch die Bereitstellung von Krankengeld. Im Laufe der Zeit wurde der Sozialstaat jedoch immer weiter ausgebaut, d. h., es wurden immer mehr Anrechte auf solidarisch finanzierte Leistungen eingeräumt und der Kreis der Anspruchsberechtigten erheblich erweitert. Ermöglicht wurde diese Entwicklung durch anhaltendes Wirtschaftswachstum und Umverteilungen. Nunmehr wird die Ansicht vertreten, daß die Anfang der 80er Jahre dieses Jahrhunderts vorherrschende Inklusionstendenz weiterer Bevölkerungskreise in die GKV zu einem weitgehenden Fortfall „unvermittelt" empfundener Solidarität geführt habe ${ }^{19}$. Es handele sich jetzt allein um eine (staatlich verordnete) Solidargemeinschaft.

Ursprünglich sollten hingegen nur diejenigen abgesichert werden, die insgesamt einer einheitlichen Berufsgruppe zuzurechnen waren. Somit handelte es sich um überschaubare Gruppen, die gemeinsame Charakteristika aufwiesen. Je kleiner eine solche Solidargemeinschaft war und je mehr sich die Mitglieder im Hinblick auf gewisse Merkmale ähnelten, um so größer war die Bereitschaft zur gegenseitigen Hilfe ${ }^{20}$. Primäres Ziel war die Absicherung des Produktionsfaktors Arbeit. Von daher fand die Solidarität ihre Grenzen im Gruppenprinzip. Aus dieser Sicht wird deutlich, daß Solidarität neben der Verfolgung individueller Interessen zur Berücksichtigung des Gemeinwohls verpflichtete. Deshalb bedeutete Solidarität keinesfalls, alles für alle bereitzustellen.

Die Umverteilung war nur solange möglich, wie Leistungsanreize verblieben und die Abgabenquote nicht eine Höhe erreichte, die die Befriedigung individueller Bedürfnisse gefährdete21. Der Sozialstaat gerät jedoch dann in Gefahr, wenn begrenzten finanziellen Möglichkeiten praktisch unbegrenzte Ansprüche gegenüberstehen ${ }^{22}$, die zwangsläufig nicht mehr befriedigt werden können.

18 Merten, in: Schulin, a.a.O., § 5 Rn. 40; BVerfGE 82, 209 ff. (230).

19 Wasem, in: Schulin, a.a.O., § 3 Rn. 136.

20 Arnold, Solidarität 2000, S. 29.

21 Arnold Ersatzkasse 9/1993, 365 ff. (366).

22 Meyer, Beiträge zur Gesundheitspolitik: Zur Neubestimmung von Solidarität und Subsidiarität im Gesundheitswesen, S. 34. 
Somit kann es nicht Ziel sein, in kollektiver Anstrengung offenbar unbegrenzt alle Bedürfnisse bei der medizinischen Versorgung zu finanzieren, wenn gleichzeitig hierdurch die Erfüllung anderer wichtiger Aufgaben gefährdet ist.

Vielfältige Ursachen haben diese Entwicklung verursacht und gefördert. An dieser Stelie soll nur ein Aspekt ausdrücklich erwähnt werden: Der Versicherte empfindet zunehmend den "pauschalen" Kassenbeitrag nicht nur als Anreiz, sondern auch als individuellen Anspruch, mehr Gesundheitsleistungen als notwendig sind nachzufragen („Moral-Hazard-Phänomen“) ${ }^{23}$. Eine derart umfassende Versicherung bewirkt im Bewußtsein des Patienten eine Verringerung des Preises von Gesundheitsleistungen für den Versicherten, der aus volkswirtschaftlicher Sicht nicht mehr mit den vollen Grenzkosten belastet wird. Folge ist, daß der Versicherte seine Nachfrage nach Gesundheitsleistungen übermäßig ausdehnt. Beitragszahlung und Leistungsinanspruchnahme haben für den Patienten nichts mehr miteinander zu tun ${ }^{24}$.

Das Solidaritätsprinzip bleibt weiterhin eine der wesentlichen Grundlagen der GKV. Mit Inkrafttreten des Gesundheitsreformgesetzes (GRG) ist dieses Prinzip des sozialen Ausgleichs ausdrücklich in $\S 1$ SGB V aufgenommen worden. In der Gesetzesbegründung heißt es auch, daß der Begriff der Solidarität anhand des GRG neu bestimmt werde ${ }^{25}$. Leistungen sind auf das medizinisch Notwendige zu beschränken und Versorgungsdefizite auszugleichen ${ }^{26}$. Die Eigenverantwortung des einzelnen wird als zweite Zielvorstellung definiert. Gemäß § 1 Satz 2 SGB V korrespondiert das Solidaritätsprinzip mit der Eigenverantwortung27. Wenn die Solidargemeinschaft im Versicherungsfall die Sachleistung bereitzustellen hat, folgt hieraus als logische Konsequenz, daß der Versicherte mit seiner Gesundheit einerseits und der Inanspruchnahme von Leistungen andererseits so umzugehen hat, daß die Solidarität nicht über Gebühr beansprucht wird. In Ergänzung findet sich im Gesetz eine Aufwertung der Gesundheitsvorsorge und der Krankheitsfrüherkennung. Es muß darum gehen,

23 Adam/Henke, in: Schulin, a.a.O., § 4 Rn. 12.

24 Sachweh, Ausnahmeregelungen für Risikopatienten in der GKV, ArztR 2/1995, S. 39 ff. (39 mitt. Sp).

25 BT-Drucks. 11/2237, S. 1 ff. (1).

26 Schulin, in: Schulin, a.a.O., § 6 Rn. 29.

27 Blaul/Biesing, Solidarität und Selbstverantwortung in der Medizin, RPG 1997, 43 ff. (45 r. Sp.). 
nicht nur die finanziellen Konsequenzen einer Krankheit abzusichern, sondern auch zu versuchen, die Wahrscheinlichkeit des Eintritts eines Schadens und sein Ausmaß zu verringern. In $\S 2$ [1] S.1, 2. HS SGB V findet sich der ausdrückliche Hinweis auf die Eigenverantwortung. Hieraus läßt sich allerdings nicht eine (von vornherein) über das geltende Recht hinausgehende Leistungseinschränkung ableiten, sondern vielmehr, „daß die GKV nicht für alle Leistungen, die der Gesundheit dienen, als Kostenträger in Frage kommt"28. Es ist daher Aufgabe, diese im Gesetz verankerten Grundsätze auch hinsichtlich des Leistungskataloges umzusetzen mit dem Ziel, den Begriff der Solidarität zu überdenken und neu zu definieren.

Hierzu bedarf es zunächst der Analyse des Begriffes und grundlegenden Inhaltes der "Solidarität". Solidarität bezeichnet die wechselseitige Verbundenheit von Menschen dergestalt, daß sie aufeinander angewiesen sind und ihre Ziele nur im Zusammenwirken erreichen können ${ }^{29}$. Wesentlicher Gedanke ist die Einbindung des einzelnen in die Gemeinschaft, weil dieser zwar seine eigenen Zwecke verwirklichen will, inm dies aber nur in Verbundenheit mit den anderen möglich ist. Überträgt man dies auf die Krankenversicherung, kann Solidarität wie folgt definiert werden:

„Allen Versicherten ist ein einheitlicher Leistungsanspruch garantiert und soll diese vor finanzieller Überforderung im Krankheitsfall schützen. Die Leistungsgewährung erfolgt dabei nach der medizinischen Notwendigkeit. Des weiteren bestimmt allein die Höhe der Beiträge die finanzielle Leistungsfähigkeit3o."

In den letzten Jahren vollzog sich ein Paradigmenwechsel im Solidaritätsprinzip. Bereits im Rahmen des Gesundheitsreform(GRG) als auch des Gesundheitsstrukturgesetzes (GSG) hat sich herauskristallisiert, daß durch die gesetzlich festgeschriebene Beitragsstabilität Leistungen eingeschränkt werden ${ }^{31}$.

${ }_{28}$ Peters, in: KassKomm, § 2 SGB V Rn. 3; Zipperer, in: GKV-Komm., § 2 SGB V Rn. 3.

29 Meyer, a.a.O., S. 14.

$30 \mathrm{Vgl}$. u.v. Langosch, Der Wettbewerb unter den gesetzlichen Krankenkassen aus rechtlicher Sicht, DOK 23-24/1995, 790 ff. (792); BT-Drucks. 11/2237, S. 1 ff. (146).

31 Bereits in der Gesetzesbegründung zum GRG wird als "Lösung" unter dem Aspekt der Neubestimmung der Solidarität ausdrücklich Leistungsbegrenzungen als auch Leistungsverbesserungen genannt. 
Und das Solidaritätsprinzip sowohl auf seiten der Finanzierung als auch auf seiten der Leistungserbringung wird weiter flexibilisiert werden müssen. Nicht zu Unrecht wird deshalb zunehmend die Ansicht vertreten, die gegenwärtige Absicherung des Krankheitsrisikos stelle keine zeitgemäße Lösung mehr dar ${ }^{32}$. Dabei ist unbestritten, daß das Solidaritätsprinzip selbst als wesentlicher Grundpfeiler der Krankenversicherung nicht in Frage gestellt werden darf. Vorrangiges Ziel muß es sein, den einzelnen zu verantwortungsvollem Umgang mit der eigenen Gesundheit zu erziehen ${ }^{33}$.

Die heute zu diskutierende Frage muß daher lauten:

"Wie weit kann und darf der Leistungskatalog der gesetzlichen Krankenversicherung eingeschränkt werden, damit das Solidaritätsprinzip als noch gewährleistet angesehen werden kann?"

\section{Möglichkeiten der Einschränkung des Leistungskata- logs}

- Ansatzpunkt ist zum einen nicht primär der Krankheitsbegriff, der vor allem aufgrund des kontinuierlichen medizinischen Fortschritts ständig weiter ausgedehnt wird, sondern die Behandlungsbedürftigkeit des einzelnen aufgrund von Krankheit ${ }^{34}$ - und dies im Licht der Bismarckschen Trias. Denn nicht ohne Grund definiert die Sozialgerichtsbarkeit "Krankheit" als regelwidrigen Körper- und Geisteszustand, der ärtlicher Behandlung bedarf35. Unerfüllbare Ansprüche stellt dagegen etwa die Europäische Sozialcharta. Danach hat jedermann das Recht, „alle Maßnahmen in Anspruch zu nehmen, die es ihm ermöglichen, sich des besten Gesundheitszustandes zu erfreuen, den

\footnotetext{
$32 \mathrm{Vgl}$. etwa Sachverständigenrat für die Konzertierte Aktion im Gesundheitswesen, "Gesundheitsversorgung und Krankenversicherung 2000", Sondergutachten 1995; Berliner Thesenpapier zur Finanzierung und damit zusammenhängenden Strukturfragen in der gesetzlichen Krankenversicherung, 1997, S. 5, Nr. 9.

33 Steinbach Gesundheitspolitik 1995, S. 3, 4; Hart, Rechtliche Grenzen der "Ökonomisierung", MedR 1996, S. 60 ff. (60 f.); Uhlenbruck, Rechtliche Grenzen einer Rationierung in der Medizin, MedR 1995, 427 ff. ( 432).

34 Uhlenbruck, a.a.O., 427 ff. (431 I. Sp.); Laufs, in: Laufs/Uhlenbruck § $1 \mathrm{Rn}$. 10.

35 BSG, 3 RK 15/65 v. 30.05.1967.
} 
er erreichen kann"36. Es erscheint aber im Sinne des Solidaritätsprinzips durchaus zulässig, sinnvoll und notwendig zu sein, „Bedürftigkeitsprüfungen“ im Hinblick auf die Aufnahme bzw. des Ausschlusses bestimmter Behandlungsmethoden, Heiloder Hilfsmittel aus dem Leistungskatalog der GKV durchzuführen. Dies gebietet auch der soziale Rechtsstaat. Denn einerseits widerspricht es seinen Prinzipien, daß Mittel der Allgemeinheit für bedürftige Bürger "mangels genügender Kontrolle auch in Fällen in Anspruch genommen werden können, in denen wirkliche Bedürftigkeit nicht vorliegt" ${ }^{37}$. Andererseits können in einer Welt endlicher Güter generelle Finanzierungsgrenzen nicht außer acht gelassen werden ${ }^{38}$. Denn niemand ist bereit, das gesamte Volkseinkommen für Gesundheit im Sinne medizinischer Versorgung aufzuwenden.

- Zum anderen ist gerade durch den ursprünglich gewährten Leistungsumfang eine Anspruchsmentalität der Gesellschaft entstanden, die trotz vielfach häufiger auftretender Risiken und deren Folgen immer weniger gewillt ist, Krankheit, Therapie, deren Folgen und ggf. bestehende Risiken als Lebensschicksal zu akzeptieren ${ }^{39}$. Wenn es sich nunmehr als unausweichlich erweist, die Erstattungsfähigkeit der bisher vom Leistungskatalog der GKV umfaßten Behandlungsansprüche des Patienten einzuschränken bzw. unter den Aspekt der Eigenverantwortung zu stellen, bedeutet dies deshalb noch keine Abkehr vom Solidaritätsprinzip und nicht die Etablierung einer Zwei-KlassenMedizin, wie dies etwa von der SPD bereits im Hinblick auf das GSG von 1993 dargestellt und hinsichtlich des 2. GKV-NOG von 1997 erneuert wurde. Ziel ist vielmehr eine Anpassung an die gegenwärtigen Verhältnisse und die finanziellen Möglichkeiten. Denn während es Bismarck ursprünglich um die Lösung der sozialen Frage ging und mit der Installation des Solidaritätsprinzips das finanzielle Eintreten der Vermögenden oder einer Gruppe für die Risiken der Unvermögenden grundlegend organisiert wissen wollte ${ }^{40}$, geht es spätestens seit Ende des

36 Europäische Sozialcharta Teil I, Ziff. 11, Teil II, Art. 11-13; Uhlenbruck, a.a.O., 427 ff. (431 I. Sp.).

37 BVerfGE 9, 20 ff. (35); 59, 52 ff. (62); Merten, in: Schulin, a.a.O., § 5 Rn. 42.

38 Oberender, in: FS-Gitter, S. $701 \mathrm{ff}$. (704).

${ }^{39}$ Ehlers/Broglie, Praxis des Arzthaftungsrechts, Kapitel 1 Rn. 5.

40 Schlenker, in: Schulin, a.a.O., § 1 Rn. 27-29; Grafe, Bedeutet die Leistungseinschränkung der gesetzlichen Krankenversicherung eine Ein- 
Zweiten Weltkrieges infolge des technischen und medizinischen Fortschritts nur noch peripher um diese Frage. Ursächlich hierfür ist nicht nur die aufgrund der demographischen Entwicklung zunehmende Morbidität, sondern es liegt $z$. T. in den durch die GKV abzudeckenden, aber bisher nie dagewesenen Risikofaktoren durch Freizeitaktivitäten.

Der dadurch mitverursachte Zielkonflikt zwischen den rechtlichen Forderungen an den medizinischen Standard ärztlicher Leistungen und der wirtschaftlichen Leistungskraft unserer Gesellschaft muß mithin durch Einschränkungen des Leistungskataloges der gesetzlichen Krankenversicherungen korrigiert werden, ohne das Solidarprinzip selbst in Frage stellen zu wollen und zu können.

Dabei stellt sich die Frage, ob oder inwiefern in der GKV Solidarität und Subsidiarität in einem Spannungsverhältnis stehen, insbesondere in bezug auf den Umfang der versicherten Leistungen. Subsidiarität, als „Zuständigkeitsregel im Sinne eines Aufbauprinzips des Handelns in der Gesellschaft" verstanden ${ }^{41}$, stellt dabei eine notwendige Ergänzung zum Solidaritätsprinzip dar. Grundsätzlich ist jedoch in der praktischen Sozialpolitik zwischen diesen Begriffen kaum Einigkeit zu erzielen. Es wird immer darum gehen, ob staatliches Handeln im Sinne des Solidaritätsprinzips erfolgen oder im Sinne einer subsidiären Zurückhaltung unterbleiben soll. So werden Leistungsbeschränkungen einerseits als Verstoß gegen das Solidarprinzip kritisiert, andererseits sind diese im Rahmen des Subsidiaritätsgedankens gerade geboten. Diskutiert man eine Leistungsbeschränkung oder eine Ausgrenzung von Leistungen aber unter dem Stichwort "Stärkung des Versicherungsprinzips", so kann Solidarität nur solche Risiken als versicherbar anerkennen, „die zufällig auftreten, von den Versicherten nicht beeinflußt werden können und alle Versicherten einer Gruppe mit der gleichen Wahrscheinlichkeit trifft42".

schränkung des Solidarprinzips, und kann der Arzt diesen Mangel kompensieren?, Arztrecht 1995, 151 ff. (151).

41 Uth, Formen und Grenzen des Subsidiaritätsprinzips, S. 2; Wasem, in: Schulin, a.a.O., § $3 \mathrm{Rn} .132$.

42 Sachverständigenrat für die Konzertierte Aktion im Gesundheitswesen "Gesundheitsversorgung und Krankenversicherung 2000", S. 103. 


\section{Ausschluß von versicherungsfremden Aufgaben}

Die gesundheitspolitische Diskussion wird bisher vorwiegend von Steuerungsmaßnahmen zur Begrenzung der Krankenversicherungsausgaben bestimmt. Die Finanzsituation der GKV wird jedoch gerade nicht nur vom Faktor "Ausgaben", sondern damit korrespondierend auch vom Faktor "Einnahmen" bestimmt. Von diesem Standpunkt aus sind Fremdleistungen für die derzeitige Situation der GKV von entscheidender Bedeutung. Während jedoch versicherungsfremden Leistungen im Rahmen der Diskussion um eine Reform der Rentenversicherung in der öffentlichen Auseinandersetzung erhebliches Gewicht zukommt, verbunden mit der Forderung, die öffentliche Hand solle die Kosten für derartige Leistungen übernehmen, wird dieses Thema in der gesetzlichen Krankenversicherung eher vernachlässigt. Schon allein aus dem Gesichtspunkt, der Versicherte solle ausschließlich und nur dafür Beiträge zahlen, was dem originären Aufgabenbereich einer Krankenversicherung zuzurechnen ist, bedarf dieses Thema der Aufarbeitung. Als Fremdleistung im engeren Sinne können dabei diejenigen nicht krankheitsbedingten Leistungen gelten, die aus gesellschafts-, sozial- und familienpolitischen Gründen der GKV übertragen worden sind, ohne daß die damit verbundenen Kosten etwa durch entsprechende Berücksichtigung bei der Beitragsberechnung übernommen wurden ${ }^{43}$. Hierzu zählen vor allem die Versorgung der Versicherten bis zum vollendeten 20. Lebensjahr mit empfängnisverhütenden Mitteln ( $§ 24$ a SGB V), Leistungen bei einer nicht rechtswidrigen Sterilisation und bei einem nicht rechtswidrigen Abbruch der Schwangerschaft, soweit dieser nicht medizinisch indiziert ist ( $§ 24$ b SGB V), Mutterschaftsgeld ( $\$ 200$ RVO), Entbindungsgeld ( $§ 200 \mathrm{~b}$ RVO), Krankengeld bei Erkrankung eines Kindes ( $§ 45$ SGB V) oder hauswirtschaftliche Versorgung im Rahmen der häuslichen Krankenpflege (§ 37 SGB V), um nur einige zu nennen ${ }^{44}$. Diese Ausgaben machten in den Jahren 1994 und 1995 bereits knapp 4 Mrd. DM aus45, teilweise wird lediglich von 2,5 Mrd. DM für das Jahr 1994 ausgegangen'6. Zum

43 Beske/Hallauer, Fremdleistungen in der GKV, Forum für Gesellschaftspolitik, Januar 1997, S. 29 ff. (29/30).

44 Vgl. etwa Uhlenbruck, a.a.O., 427 ff. (431 r. Sp.); Sachweh, a.a.O., 39 ff. (40/41); Gutachten des Sachverständigenrat, a.a.O., S. 103/104; Beske/Hallauer, a.a.O., S. 29 ff. (30).

45 Sachverständigenrat, a.a.O., S. 104 (Tabelle 4); Beske/Hallauer, a.a.O., S. 30.

46 Berliner Thesenpapier, a.a.O., S. 4, 2.Abs. 
Teil wird die Ansicht vertreten, auch weitergehende, nicht direkt krankheitsbezogene Leistungen wie das Sterbegeld ( $\$ 58$ f SGB V), Kuren als auch ambulante und stationäre Vorsorgekuren, kosmetische Operationen, Rehamaßnahmen, Teile der Prophylaxe und Prävention, Maßnahmen der Gesundheitsförderung sowie die Pauschalierung von Leistungen, etwa der Physiotherapie, könnten aus dem Leistungskatalog der GKV ausgeschlossen oder zumindest eingeschränkt werden ${ }^{47}$. Dabei hat der Hartmannbund für 1992 noch erheblich höhere Summen errechnet. So betrugen die Ausgaben für Sterbegeld, Schwangerschaftsabbrüche, Sterilisation und Mutterschaftsleistungen allein 4,9 Mrd. DM, für Kuren 2,9 Mrd. DM und für Folgekosten der familienbezogenen Leistungen 1,2 Mrd. DM ${ }^{48}$. Zu berücksichtigen ist hierbei aber, daß es sich beim Sterbegeld um eine auslaufende Leistung handelt und der Gesetzgeber mit den $\S \S 58 \mathrm{ff}$. SGB V eine erhebliche Begrenzung des Anspruchs in der Höhe gegenüber der Rechtslage vor Inkrafttreten des GRG festgelegt hat ${ }^{49}$.

Grundsätzlich kann Aufgabe von Krankenversicherungen also weiterhin nur sein, krankheitsspezifische Risiken abzudecken, nicht jedoch darüber hinausgehende Aufgaben zu erfüllen. $\mathrm{Ob}$ auch medizinische Maßnahmen zur Herbeiführung einer Schwangerschaft ( $\$ 27$ a SGB V), insbesondere die homologe Invitro-Fertilisation (IVF) unter die Gruppe der krankenversicherungsfremden Leistungen zu zählen sind, bleibt dagegen umstritten. So hat der BGH den subjektiven Leidensdruck und den drängenden Wunsch nach einem leiblichen Kind durchaus als "Krankheit" und auch als medizinisch notwendig angesehen ${ }^{50}$ und jedenfalls für den zweiten und dritten Versuch eine Einstandspflicht der PKV angenommen51. Wenn dies aber als „Krankheit" anzusehen ist, kann auch im Hinblick auf den ursprünglichen Zweck der GKV an sich nichts anderes gelten. Ein krankheitslindernder oder bei Erfolg sogar krankheitsheilender Charakter der IVF kann zumindest nicht grundsätzlich verneint werden. Dennoch wird diese in Teilen der Literatur als krankenversicherungsfremde Leistung angesehen ${ }^{52}$. Es bietet sich ein

\footnotetext{
47 Sachweh ArztR 2/1995, 39 ff. (41 I. Sp.).

48 Sachweh, a.a.O., S. 41.

49 Sachverständigenrat, a.a.O., S. 106.

50 BGH NJW 1987, 703; Selb JZ 1987, 622 ff. (625); Laufs Arztrecht, Rn. 384, 388; ders., Fortpflanzungsmedizin und Arztrecht, S. 60 ff. (62).

51 BGH MedR 1988, 34 f.

52 Sachweh, a.a.O., S. 41 I. Sp.; Sachverständigenrat, Sondergutachten
} 
Vergleich mit kosmetischen Operationen an. Denn hier fallen ohnehin nur diejenigen Behandlungen unter den Begriff der Krankheit und der ärztlichen Heilbehandlung, wenn der körperlich Verunstaltete seelisch leidet ${ }^{53}$, keinesfalls jedoch bei schlichter Verschönerung des äußeren Erscheinungsbildes. Selbst wenn einleitend die Ansicht vertreten wurde, Aufgabe der Krankenversicherung sollte ausschließlich die Abdeckung krankheitsspezifischer Risiken sein, müssen bezüglich der Einbeziehung von Maßnahmen der Gesundheitsförderung, Früherkennung und Prävention gewisse Einschränkungen vorgenommen werden 54 : Zwar ist die Gesundheitsförderung nach der obengenannten Eingrenzung keine "versicherbare Leistung" im oben definierten Sinn, obliegt somit grundsätzlich anderen Trägern. Jedoch sollten zur Vermeidung einer zu starken politischen Einflußnahme bestimmte Präventionsmaßnahmen weiterhin im Aufgabenbereich der GKV verbleiben.

Mithin zeigt sich, daß in der über 100jährigen Geschichte der GKV etliche sozial- und familienpolitische Aufgaben durch die GKV übernommen worden sind, die keinesfalls mehr unter den Begriff der „Krankheit" subsumiert werden können. Nicht zuletzt aus ordnungspolitischen Gründen könnten diese Bereiche von anderen Trägern übernommen werden. Fremdleistungen sollten somit entweder vollständig aus dem Leistungskatalog gestrichen oder der gesetzlichen Krankenversicherung lediglich als Auftragsverwaltung - verbunden mit voller Kostenerstattung - übertragen werden ${ }^{55}$. Als weitere und ergänzende Möglichkeit ist für einige Leistungen auch eine Zusatzversicherung in Betracht zu ziehen ${ }^{56}$.

Weiterhin problematisch wird sich aufgrund der demographischen Entwicklung die Krankenversicherung der Rentner gestalten. Denn die Schere zwischen den Beiträgen der Rentner und den Ausgaben für deren Krankenversicherung wird sich weiter vergrößern. In diesem Zusammenhang wird eine Diskussion notwendig sein, da eine Entlastung der GKV dringend geboten ist und die kommende Generation weder in der Lage noch bereit sein wird, derart hohe Versicherungsbeträge zu zahlen, so daß

1995, a.a.O., S. 103.

53 Laufs, Arztrecht, Rn. 29.

54 Sachverständigenrat, a.a.O., S. 107.

55 Beske/Hallauer, a.a.O., S. 31 r. Sp.

56 Sachverständigenrat, a.a.O., S. 104. 
das verfügbare Einkommen weit über ein vertretbares Maß hinaus eingeschränkt würde. Folglich muß auch diese Art von Subventionierung der Rentner in diesem Umfang bereits als „krankenversicherungsfremde Entwicklung" 57 angesehen werden.

\section{Ausschluß weiterer nicht spezifischer Krankheitsrisiken}

Genauso zählen hierzu, wie bereits angedeutet, nicht spezifische Krankheitsrisiken, so etwa Früherkennung, Maßnahmen zur Verhütung von Krankheiten und arbeitsmedizinische Untersuchungen, die, wie der Ersatz von Brillen, Schönheitsoperationen und Kuren, nicht unter den verfassungsrechtlichen Schutz der Gesundheit fallen ${ }^{58}$. Beim Ersatz von Brillen sind größtenteils Eigenbeteiligungen ohnehin schon lange der Fall. Denn nach $\S 33$ [4] SGB $V$ besteht lediglich hinsichtlich der Versorgung mit Brillengläsern, nicht jedoch bezüglich Brillengestellen eine, wenn auch keinesfalls umfassende Naturalleistungspflicht in Form von Zuzahlungen. Schönheitsoperationen betreffen zweifelsohne sachfremde Aufgaben, mit einer Einschränkung für diejenigen Fälle, in denen psychische und seelische Belastungen aufgrund von angeborenen oder unfallbedingten Mißbildungen durchaus noch unter den Krankheitsbegriff fallen können. Bei Kuren, die die Kassen der Krankenkassen erheblich belasten und zudem eher der Gesundheitsvorsorge dienen als dem verfassungsrechtlich garantierten Recht auf Gesundheit und Heilung, besteht allemal der Verdacht, eine ausufernde Versichertenmentalität zu fördern. Denn wer vermeintlich zu viel an Beiträgen zahlt, will auch mehr zurückerhalten - trotz oft zweifelhafter medizinischer Indikation.

\section{Sonderfall der Sportverletzungen}

Innerhalb dieser Ausschlußgruppe von nicht spezifischen Krankheitsrisiken nimmt die bisherige Abdeckung von Risiken, die aus verletzungsgefährdeten, riskanten und extravaganten Sportarten resultieren, eine besondere Stellung ein. Deshalb stehen bereits besondere Versicherungen für spezielle Risiken zur Diskussion. Dies gilt nicht nur für Sportverletzungen, sondern auch für Raucher, Personen, die dem übermäßigen Konsum von Alkohol nicht entsagen wollen oder ähnlich ungesunde Lebensweisen. (Dies ist durch Einführung eines „Bonusheftes" hinsichtlich der Zahnkon-

57 Beske/Hallauer, a.a.O., S. 31 I. Sp.

$58 \mathrm{Vgl}$. etwa Uhlenbruck, a.a.O., S. 432 I. Sp. 
trolle bei der gesetzlichen Krankenversicherung üblich, um später einen möglicherweise notwendigen Anspruch auf Zahnersatz geltend machen zu können.)

Von der Gegenseite wird der Vorschlag vornehmlich mit dem Argument abgelehnt, dadurch könnten sich nur noch die Vermögenden gewisse sportliche Aktivitäten leisten und es würde einer Rationierung nach dem Verursacherprinzip quasi gleichzusetzen sein. Dies ist nicht zutreffend; denn während eine Rationierung nach dem Verursacherprinzip schon deshalb nicht zulässig wäre, da diese denjenigen benachteiligen würde, der aufgrund seiner genetischen Veranlagung gezwungen ist, häufige und $z$. T. teure Behandlungen über sich ergehen zu lassen59, liegen bei Ausübung von Sportarten völlig andere Voraussetzungen vor. Sie fallen allesamt in den Bereich der beeinflußbaren Risiken. Zum einen wird gerade nicht, wie es bei einer Rationierung nach dem Verursacherprinzip der Fall wäre, die ärztliche Schweigepflicht unterlaufen, da bestimmte Risiken gesondert versichert wären, damit der Arzt nicht gezwungen wäre, selbstverschuldete Krankheitsursachen zu offenbaren.

Zum anderen könnte auf ähnliche Voraussetzungen der Kraftfahrzeugversicherung abgestellt werden, die ebenfalls als alltäglich und notwendig erachtet wird. Denn diese haftet für das bloße Halten eines Kraftfahrzeugs auf der Grundlage des $\S 7$ [1] StVG und die daraus resultierenden möglichen betriebsspezifischen Gefahren. Hiergegen wiederum wird eingewandt, die Autohaftpflichtversicherung trete lediglich für fremden Schaden bei einem verschuldeten Unfall ein, während die Krankenversicherung lediglich die Sorge um den eigenen finanziellen Schaden im Krankheitsfall nimmt60. So sei die Kraftfahrzeugversicherung nur eine differenzierte Ausgestaltung der $\S \S 61,62$ WG, wonach der Versicherer von der Verpflichtung zur Leistung jedenfalls dann frei ist, wenn der Versicherungsnehmer den Versicherungsfall vorsätzlich oder grob fahrlässig herbeiführt oder seiner Schadenminderungspflicht nicht nachkommt. Vielmehr wird vorgeschlagen, insbesondere den Rechtsgedanken des § 62 VVG auf die Krankenversicherungsnehmer zu übertragen. Danach wären insbesondere Suchtkranke, die nach erfolgreicher Entziehung ihr bisheriges Umfeld nicht meiden wollen, erneut rückfällig werden

59 Uhlenbruck, a.a.O., S. 432.

60 Blaul/Biesing, a.a.O., 43 ff. (46). 
und infolgedessen eine erneute Entziehungskur benötigten, genauso wie ungeübte, risikobereite Sporttreibende durch ihr Verhalten von einer derartigen Regelung betroffen 61 . Jedoch bergen Sportarten jeglicher Art gewisse unvermeidbare Risiken, genauso wie bei entsprechender Konstitution ein dosierter Alkoholkonsum durchaus positive gesundheitliche Auswirkungen haben kann. Ohnehin ist nach $\S 52$ SGB V eine Leistungsbeschränkung bei Selbstverschulden, insbesondere Vorsatz, vorgesehen, wenn dieses auch häufig an Feststellungsschwierigkeiten scheitert. Eine der Kfz-Versicherung ähnlich geartete Versicherung soll aber schon auf eine umfassende Versicherungspflicht vor dem Betreiben einer Sportart abzielen, insbesondere bei gefährlicheren Sportarten wie Skifahren, Tiefseetauchen oder Bungee-Springen. Aufgrund der aus der vorgeschlagenen Regelung resultierenden Beweislastumkehr wäre es zudem dem Sportler häufig unmöglich, den Anscheinsbeweis der Versicherung zu erschüttern. Eine Ausgliederung aus der GKV und die Einführung einer einheitlichen Zusatzversicherung erscheint nicht zuletzt aus ordnungspolitischen Gründen vorzugswürdig. Es besteht mithin kein Argument, weshalb dies nicht auch für das Betreiben von Sportarten bzw. die bloße Möglichkeit des Betreibens durch Besitz des notwendigen Materials hierzu möglich sein sollte. Dies würde die Krankenkassen entlasten und es den einzelnen Ärzten und den Krankenhäusern ermöglichen, nicht ständig aufgrund von Budgetierung, Sonderentgelten, Fallpauschalen und Richtgrößen den an sich bestehenden Nachrang des Wirtschaftlichkeitsgebotes möglicherweise unberücksichtigt zu lassen. Bei vielen Sportarten, etwa Skifahren, ist diese Gefahr und die damit möglicherweise verbundenen erhöhten Kosten - zumindest im Ausland - ohnehin schon (teilweise) durch die Auslandskrankenversicherung abgedeckt. Ob somit eine von der Gegenseite behauptete Verletzung des Gleichheitsgrundsatzes nach Art. 3 GG vorliegt, mag ernsthaft bezweifelt werden.

Da eine Tendenz zu immer gewagteren und vor allem immer häufiger praktizierten Sportarten in der heute zweifelsohne bestehenden "Freizeitgesellschaft" unverkennbar ist, wird ein Ausschluß dieser Krankheitsrisiken langfristig nicht zu vermeiden sein. Über andere Modelle der Finanzierung, etwa Abgaben auf Produkte, könnte zudem nachgedacht werden.

61 Blaul/Biesing, a.a.O., S. 47 I. Sp. 
Durch den Ausschluß dieser Risiken aus dem Leistungskatalog der GKV würden diese einem anderen Rationierungsverfahren, dem Preisausschlußverfahren, überantwortet62. Das Rationierungskriterium des Preisausschlußverfahrens stellt dabei die individuelle Zahlungsbereitschaft dar. Die Kapazitäten kämen dann nämlich den zahlungsbereitesten, nicht unbedingt den einkommensstärksten Patienten zugute. Durch die entstandene Anspruchsgesellschaft in der heutigen Zeit würde dies einen sicher besonders schmerzhaften Einschnitt darstellen. Jedoch auch heute gewährleistet die GKV lediglich eine, wenn auch umfangreiche Grundversorgung. Darüber hinausgehende individuelle Gesundheitsbedürfnisse müssen in großem Umfang mit privaten Zusatzversicherungen oder direkten Zuzahlungen befriedigt werden oder sogar von vornherein unberücksichtigt bleiben.

Mit dem scheinbaren Ausschluß des Kernpunktes des Solidaritätsprinzips läßt sich von seiten der Gegner dieser Auffassung dagegen nur schwerlich argumentieren. Vielmehr kann es gerade nicht Sinn und Zweck des Solidarprinzips sein, übermäßig hohe Risiken und damit möglicherweise verbundene Kosten eines relativ kleinen Teils der Bevölkerung zu Lasten der Unvermögenden abzurechnen. Es erscheint dagegen angemessen, eine starre und einseitige Ablehnung von Beschränkungen des Leistungskatalogs der gesetzlichen Krankenversicherung kritisch und objektiv zu überdenken.

Ähnliche Überlegungen gelten in gleicher Weise für die Absicherung des Krankheitsrisikos bei Auto- und Motorradunfällen. Voraussetzung hierfür, um den Versicherungsfall klar definieren zu können, ist ein eindeutiger Zusammenhang zwischen individuellem Verhalten und Risikoeintritt63. Dieser ist zumindest bei Unfällen mit Personenschäden eindeutig erfüllt, da das versicherte Verhalten, d. h. die Benutzung eines Autos oder Motorrades, ursächlich für die Gesundheitsschäden des Unfallverursachers ist.

\section{Ausschluß von Bagatellerkrankungen}

Eine weitere Möglichkeit der Beschränkung des Leistungskataloges stellt schließlich der Ausschluß von Bagatellerkrankungen dar. Dies betrifft vornehmlich regelmäßig wiederkehrende Erkran-

62 Oberender, in: FS-Gitter, S. 709, 2. Abs.

63 Sachverständigenrat, a.a.O., S. 108 ff. (108). 
kungen, die etwa jahreszeitbedingt gehäuft auftreten, bei denen der Hausarzt mithin nur zum Erhalt des notwendigen Rezeptes aufgesucht wird. Da jedoch spätestens seit Inkrafttreten der 3. Stufe der Gesundheitsreform, wenn nicht schon davor, mit stark steigender Tendenz besonders Arzneimittel eben für die angesprochenen Erkrankungen ohne vorherigen Arztbesuch gekauft werden, könnte sich auch ein Ausschluß von Bagatellerkrankungen als Möglichkeit der Einschränkung des Leistungskatalogs anbieten. Die Frage, ob die hinsichtlich der erhöhten Zuzahlung für Medikamente getroffene Regelung gegenüber einer ebenfalls diskutierten Negativ- oder Positivliste vorzuziehen ist, soll an dieser Stelle dahinstehen. Problematisch könnte es allenfalls für den einzelnen Patienten sein, Bagatellerkrankungen von ernstzunehmenden und durch einen Arzt behandlungsbedürftigen Erkrankungen zu unterscheiden. Auch bei älteren Patienten könnte dies problematisch sein, wenn sich aus anfänglichen Bagatellerkrankungen ernstere Erkrankungen entwickeln. Im Zweifel wird sich der Patient jedoch immer an einen Arzt wenden, um Gewißheit zu erlangen. Somit erscheint ein Ausschluß von Bagatellerkrankungen - zumindest in begrenztem Umfang - als Möglichkeit der Einschränkung des Leistungskataloges der GKV überdenkenswert.

\section{Zusammenfassung}

Es lassen sich durchaus Möglichkeiten einer weiteren Einschränkung des Leistungskatalogs der GKV nach den eben dargelegten Kriterien feststellen, ohne das Solidaritätsprinzip zu vernachlässigen oder sogar in sein Gegenteil zu verkehren. Vielmehr erscheint es in der heutigen Anspruchsgesellschaft sogar grundsätzlich heilsam zu sein, durch eine hierdurch motivierte natürliche Stärkung der Eigenverantwortung des einzelnen als eines der Kernpunkte der Bismarckschen Trias, die nur zu gerne hinter das Solidaritätsprinzip zurückgedrängt wird, zu einer erheblichen Kostensenkung und einer damit verbundenen Minderung des bestehenden Kostendrucks im Gesundheitswesen beizutragen. 


\section{Anmerkungen zum Solidaritätsprinzip}

Herwig F. Schirmer

Obwohl ich umgeben bin von methodisch arbeitenden Wissenschaftlern, habe ich mich entschlossen, nicht einfach das vorzulesen, was mir meine Mitarbeiter korrekt aufgeschrieben haben. Statt dessen möchte ich einige Anmerkungen zu dem machen, was jemanden, der an der "Sozialfront" tätig ist, in seinem beruflichen Alltag bedrängt und natürlich sehr stark mit dem Solidaritätsprinzip zusammenhängt.

Ohne das Solidaritätsprinzip gibt es keine gesetzliche Krankenversicherung, die ja darauf beruht, daß junge und gesunde Menschen für die Alten und Kranken einstehen, ebenso wie die Besserverdienenden für die sozial Schwachen. Die Frage ist nicht, ob wir eine solidarische gesetzliche Krankenversicherung brauchen. Das sollte für alle, die den sozialen Frieden in Deutschland halten wollen, selbstverständlich sein. Die Frage ist vielmehr, ob wir sie halten können oder ob der wirtschaftliche und soziale Druck in der Gesellschaft so stark wird, daß uns mit dem Solidaritätsprinzip die GKV verlorengeht.

Ich bin der Überzeugung, daß jede Gesellschaft so etwas wie eine solidarische Gesinnung oder Solidarhaltung braucht. Wir benötigen ja nicht nur in der GKV ein Solidarsystem, sondern auch anderswo. Die Kirchen sind von ihrem Selbstverständnis, von der christlichen Idee her nicht zuletzt auch eine solidarische Einrichtung. Ich habe mit unserem Landesbischof über die finanzielle Lage der Evangelischen Kirche Berlin-Brandenburg gesprochen. Dort sind die Einnahmen, die ja an der Einkommenssteuer hängen, in den letzten Jahren um $40 \%$ zurückgegangen. Die Kirche weiß kaum noch, wie sie ihren sozialen Hilfsauftrag erfüllen soll. Die Personalabbauprogramme sind erheblich. Die sozialen Hilfseinrichtungen der Kirche kommen bei der Bewältigung der Anforderungen kaum noch mit. Flächenhaft bricht zum Beispiel die Krankenhaus- und Gefangenenseelsorge weg. Ich empfehle Ihnen, sich einmal die Arbeit, die diese Leute vor Ort an der "Sozialfront" leisten, konkret anzuschauen, um zu begreifen, in welch innerem Zustand sie zur Zeit ihre Arbeit verrichten. Das fast lähmende Gefühl des Alleingelassenwerdens mit dem sozialen Elend ist mit den Händen greifbar. 
Eine weitere solidarische Institution, die in diesem Zusammenhang eine Rolle spielt, sind die Gewerkschaften. Auf unserer Tagung ist die relative Machtlosigkeit der Gewerkschaften eindrucksvoll belegt worden. Darüber kann man sich kaum freuen. Es gibt sicher ein paar Leute, die sagen, daß es endlich an der Zeit ist, die Gewerkschaften und die sie vertretenden Funktionäre aus ihrer Machtrolle herauszudrängen. Ich befürchte aber, daß mit dem Niedergang der Gewerkschaften auch wichtige Transformatoren für die Solidaritätsidee in einer Weise fehlen werden, die der Gesellschaft insgesamt gar nicht bekommt. Mit einer Erosion des Tarifvertragssystems wäre ohne Frage auch die Zerstörung des Solidarprinzips ein großes Stück vorangeschritten.

Ich bin fest davon überzeugt, daß wir eine moralische Fundamentierung der Politik benötigen, um der schleichenden Entsolidarisierung in unserer Gesellschaft Einhalt zu gebieten. Es ist zwar nötig, daß wir Kostenbewußtsein in unserer Gesellschaft entfalten. Ich halte es aber für falsch, das Betriebswirtschaftlichkeits-Know-how internationaler Beratungsgesellschaften zum Leitgedanken der Politik zu machen. Wir erleben eine fatale Diskriminierung des Begriffes "Soziales". Ich habe den Eindruck, daß die meisten, die so leichtfertig über die Notwendigkeit einer Reduzierung des Sozialstaates reden, gar nicht wissen, was sich hinter dem Sozialstaat alles verbirgt. Das hängt natürlich auch mit dem unerträglich gewordenen Lagerdenken in der Bundesrepublik zusammen, wo man sich anschickt, alle Brücken zwischen Ökonomie und Sozialem abzureißen, obwohl die gesamten Sozialaufwendungen gigantische ökonomische Bedeutung haben.

Der weitaus größte Teil dieser Sozialaufwendungen wird für wirklich Beladene ausgegeben. Trotz dieser Tatsache wird allenthalben über die "soziale Hängematte" und "Abstaubermentalität" geredet und der Sozialpolitiker als "Sozialheini" verächtlich gemacht. Mit der Wirklichkeit hat das alles nichts zu tun. Die Wirkung dieser Mentalität auf den Sozialbereich ist verheerend. Was meinen Sie, was für Ängste in den Behinderteneinrichtungen, Altenpflegeheimen und anderen sozialen Betreuungseinrichtungen entstehen, wenn sie diese Sprüche tagtäglich in den Medien über sich ergehen lassen müssen? Ich empfehle Ihnen dringend, sich einmal eine Behinderteneinrichtung genau anzuschauen und sich ein Bild von der Stimmung bei den Mitarbeitern und Betroffenen zu machen. Ich habe große Sorge, daß man über all die Diskussionen um Steuerreform, Lohnnebenkosten etc. diese Probleme völ- 
lig aus den Augen verliert. Dabei müßte doch begreifbar sein, daß der Arbeitsmarkt zu einem großen Teil von menschnahen Dienstleistungen gestützt wird, die der Sozialstaat garantiert.

Natürlich ist die Arbeitslosigkeit das Hauptproblem für die Finanzierung des Sozialstaates. Arbeitslosigkeit ist nicht nur in finanzieller Hinsicht eine Gefahr für das Solidarprinzip. Solidarität setzt ein Mindestmaß an sozialer Sicherheit voraus. Es ist ein Irrglaube anzunehmen, daß sozial Schwache wirklich auf Dauer solidarisch sein können, wenn sie selbst um ihre eigene Existenz täglich neu ringen müssen. Diejenigen, die sich noch in materiell gesicherten Verhältnissen bewegen, müssen hier ihre Verantwortung deutlich sehen und dabei beachten, daß ein positiver Wettbewerb in der Wirtschaft keinesfalls gleichgesetzt werden kann mit einer Rechtfertigung aller Art persönlicher Vorteile.

In diesem Zusammenhang möchte ich auch das Problem des Wettbewerbes in der gesetzlichen Krankenversicherung ansprechen. Wir sind ohne Frage auf einem Weg, durch den Wettbewerb das Solidaritätsprinzip zu bedrohen. Wenn es so ist, daß die Zahl der "schlechten Risiken" ein entscheidendes Wettbewerbskriterium wird oder sein kann, dann müssen wir davon ausgehen, daß eine Geschäftspolitik zwangsläufig Platz greift, die auf die Ausgrenzung eben dieser "schlechten Risiken" hinausläuft. Als ehemaliger Krankenkassenvorstand weiß ich, wovon ich rede. Gott sei Dank gibt es gegen eine solche Entsolidarisierung in der GKV erhebliche Widerstände, aber ich warne davor, daß eine neue Generation von Leuten Verantwortung übernimmt, die ihren Beruf nicht als sozialpolitischen Auftrag, sondern als rein kaufmännische Aufgabe ansieht, bei der das Scheitern von Mitbewerbern zum gewohnten und gewollten Alltagsgeschehen gehört. Hier muß der Staat Wettbewerbsbedingungen wie den Risikostrukturausgleich schaffen, um einen Wettbewerb, um bessere Ideen zu gewährleisten.

Der Staat hat eine wichtige Aufgabe zur Sicherung des Solidaritätsprinzips. Ich bin der festen Überzeugung, daß es keine Zwangsbeglückung ist, eine gesetzliche Krankenversicherung mit Pflichtcharakter zu haben. Es ist eine Illusion zu glauben, daß jemand, der 1.800 DM netto im Monat nach Hause trägt und eine vierköpfige Familie ernähren muß, in der Lage ist, freiwillig eine Langfristvorsorge für die Sicherung im Krankheitsfall zu organisieren. Daran hat sich seit der Zeit von Bismarck bis heute nichts 
geändert. Der Staat muß ein Stück Verantwortung in der Garantie für solidarisches Verhalten übernehmen, wenn wir die gesetzliche Krankenversicherung und den Sozialstaat nicht ganz aufgeben wollen.

Ich bedaure auch die Art und Weise, wie der Staat zur Zeit in diesem Zusammenhang eingestuft wird. Wir laufen Gefahr, daß der Staat in immer schwieriger werdenden Zeiten in seinem Ansehen unangemessen beschädigt wird, wenn man ihn nur als einen bürokratischen Popanz darstellt. Auch die Wirtschaft muß begreifen, daß sie einen funktionierenden Staat braucht, der bestimmte Dinge für sie erledigt und dabei auch Unterstützung erwarten kann. Ich befürchte, daß wir an diesem Punkt eine eher schädliche Debattenlage haben, wie ich auch glaube, daß die globale Abwertung von Politikern ausgesprochen oberflächlich ist.

Ich bin überzeugt davon, daß die Menschen in unserer Gesellschaft nur solidarisch sein können, wenn sie wissen, worum es geht. Die gesetzliche Krankenversicherung hat einen hohen Grad von Akzeptanz, und wir müssen verhindern, daß der Eindruck entsteht, dort seien nur "Absahner" und "Beutelschneider" am Werk. Das ist nicht nur eine Frage des Ansehens, das untergräbt auch die Fundamente der gesetzlichen Krankenversicherung.

Ich bin überzeugt davon, daß wir alle mehr tun müssen, um bei den Bürgern die Akzeptanz für das soziale Sicherungssystem zu stärken. In dieser Frage haben auch die Sozialpolitiker selbst Fehler gemacht. Sie ruhen viel zu sehr in sich selbst, bestätigen sich gegenseitig immer wieder, wie gut und wie wichtig sie sind; aber sie schlagen zu wenig Brücken zu anderen gesellschaftlichen Lagern. Dabei ist es gar nicht so schwer, sich als Sozialpolitiker verständlich zu machen. Wenn Sie mal in einem Lions Club einen Vortrag über Sozialpolitik halten und den mit dem - natürlich dämlichen - Satz beginnen „Damit Sie es wissen: Im Laufe Ihres Lebens werden Sie entweder tot oder ein Pflegefall sein", dann werden Sie bei Ihren Zuhörern eine ganz andere Einstellung zu diesen Dingen finden, als wenn sie langatmige Ausführungen über das Sozialbudget machen. Ich habe den Eindruck, es gibt bei uns eine "Wegseh-Gesinnung", die letztendlich zu Unsicherheiten der Menschen führt, die nicht in der Sozialpolitik tätig sind. Diese Wegseh-Gesinnung muß durch versöhnlichen Umgang mit anderen gesellschaftlichen Gruppen beseitigt werden. 
Auch Überforderung zerstört Solidarität. Wenn derjenige, der solidarisch sein soll, ständig das Gefühl hat, daß er auch wegen Nichtigkeiten in Anspruch genommen wird, dann ist das ein Weg in die Entsolidarisierung. Ich nehme in diesem Zusammenhang in Kauf, daß jetzt jemand sagt, der Staatssekretär Schirmer ist für Kern- und Wahlleistungen $u$. dgl. Das ist gar nicht mein Problem. Wir brauchen eine Straffung auf das Wesentliche, wenn wir Solidarität einfordern wollen. Ich bin der festen Überzeugung, daß Solidarität auch heißen muß, die Tüchtigkeit des einzelnen zu würdigen und nicht zu bestrafen.

Wesentlich ist allerdings, daß das Solidarsystem vor allem dann gefährdet wird, wenn der Solidarhaltung der kleinen Leute schlechte Vorbilder bei den wirtschaftlich Starken gegenüberstehen. Ich halte es für auf die Dauer nicht vermittelbar, daß Gutverdienende nicht oder nur eingeschränkt am sozialen Sicherungssystem der kleinen Leute mitbeteiligt sind. Ebensowenig ist es vertretbar, daß vor allem sehr Reiche bislang alle Möglichkeiten haben, ihre Steuerlast effektiv gegen Null zu drücken. Und ich halte es auch für hochgefährlich zu glauben, daß die kleinen Leute das nicht merken. Sie noch länger mit der Phantomhoffnung ruhigzustellen, daß man die Reichen reicher machen muß, damit die kleinen Leute wieder Arbeit bekommen, muß scheitern. Eine wirkliche Steuerreform, bei der die Absenkung des Spitzensteuersatzes nur eine Bagatelle ist, ist nicht zuletzt zur Erhaltung des Solidargedankens dringender denn je.

Das Solidaritätsprinzip in der gesetzlichen Krankenversicherung setzt auch straffe Organisationsstrukturen voraus. Da ist eine Menge passiert in den letzten Jahren, woran ich teilweise selber beteiligt war. Wir müssen auch die Wirtschaftlichkeit des Versorgungssystems in den Griff bekommen, damit nicht das Vorurteil weitere Nahrung erhält, daß da Abzocker am Werk sind, für die dann die Versicherten monatlich $800,--$ DM bezahlen müssen. Wir müssen meiner Meinung nach eine neue Sortierung steuerfinanzierter und beitragsfinanzierter Elemente in der gesetzlichen Krankenversicherung zustande bringen. Das ist gerade von den Wissenschaftlern überzeugend dargelegt worden. Wir benötigen auch eine verbesserte Form der steuerlichen Begünstigung derer, die sich heute noch die Last auferlegen, Arbeitsplätze einzurichten und zu organisieren. 
Es geht auch nicht, daß jeder, der sich über die Differenzierung von Leistungen Gedanken macht oder über Selbstbeteiligung redet, gleich mit Totschlagargumenten wie "Zwei-Klassen-Medizin“ konfrontiert wird. Auch hat das Engagement für das Solidarprinzip und die soziale Krankenversicherung nichts mit "totaler Gleichmacherei" zu tun. Wir müssen endlich vernünftig mit diesen Dingen umgehen und aufhören, uns gegenseitig immer nur mit Klischees zu traktieren.

Ich warne davor, die GKV zu einer Privatversicherung zu machen und zu glauben, daß dann die Welt in Ordnung ist. Auch hier könnte man ja vielleicht von eingefahrenen Diskussionsritualen ein bißchen wegkommen, indem man den Spielraum der privaten Krankenversicherung an der einen Stelle erweitert, zugleich aber die Besserverdienenden und Beamten etwas stärker in die $\mathrm{Fi}$ nanzierung der gesetzlichen Krankenversicherung mit einbezieht. Wir müssen weg von diesen Schablonendiskussionen, die uns jahrzehntelang begleitet haben und die man heute nicht mehr hören kann. Man sollte endlich wieder sachbezogen miteinander umgehen, und das gilt natürlich vor allem für die Politik. Wir müssen runter von diesen Blockadehaltungen, von dem Lagerdenken, weil wir sonst in der Gesundheitspolitik nicht weiterkommen. Eine vernünftigere Form der gesetzlichen Krankenversicherung kann nicht zustande kommen, wenn die Meßlatte der dafür gemachten Gesetze die Zustimmungsfreiheit im Bundesrat ist.

Bismarck war glücklicherweise nicht nur für die Sozialistengesetze verantwortlich, sondern auch für die Schaffung der gesetzlichen Krankenversicherung. Es steht uns gut zu Gesicht, dieses Erbe eines konservativen Politikers nicht kaputtzumachen, bevor wir was Besseres entwickelt haben. 


\section{Reformoptionen im Gesundheitswesen - Der Leistungskatalog der GKV im Lichte des Solidar- prinzips \\ Neuordnung des Leistungskataloges der GKV als Reformoption?}

Dominik Graf von Stillfried

\section{Zur Themenstellung}

Unter den drei Themenkreisen dieser Veranstaltung dürften die Fragen, ob und welche Anpassungen des Leistungskataloges künftig erforderlich sind, und wie diese ggf. vorgenommen werden sollten, die schärfsten Kontroversen auslösen.

Noch vor kurzem war dieses geradezu ein Tabuthema. Jede Beschäftigung mit der Problematik setzte den Betreffenden dem Vorwurf aus, er befürworte das Prinzip der Rationierung. Langsam wird jedoch eine Öffnung der Diskussion erkennbar.

Angesichts des immer enger werdenden Finanzierungsspielaums der GKV werden vor allem von seiten der Leistungserbringer zunehmend Forderungen nach einer Überprüfung des Pflichtleistungskataloges laut64. Von Kostenträgerseite wird dies bisher mit dem Hinweis abgelehnt, zuerst müßten unnötig kostentreibende und strukturell bedingte Defizite in der Versorgung abgebaut werden ${ }^{65}$. Einer Verlagerung der sogenannten versicherungsfremden Leistungen aus der Leistungspflicht der GKV in eine Finanzierung aus dem allgemeinen Steueraufkommen zeigen sich die Kran-

$64 \mathrm{Vgl}$. z. B. die Überlegungen zu individuellen Gesundheitsleistungen der KBV („Ausgegliederte GKV-Leistungen sollen den Vertragsärzten und Kassen helfen“, Ärzte-Zeitung vom 05.11.1996), die Position des Hartmannbundes („Hartmannbund fordert mehr Eigenvorsorge", Handelsblatt vom 27.10.97) oder das Symposium der Kassenärztlichen Bundesvereinigung zum Thema Neuordnung des Leistungskataloges der Gesetzlichen Krankenversicherung, 20./21.10.1997.

$65 \mathrm{Vgl}$. z. B. Vortrag von Johann-Magnus von Stackelberg auf dem KBVSymposium, Neuordnung des Leistungskataloges der Gesetzlichen Krankenversicherung, 20./21.10.1997 (Manuskript); Fiedler E., Mit Hokuspokus gegen Schnickschnack? Forum für Gesellschaftspolitik 2 (9) 1996:217-220; und weiter zurückliegende Diskussionsbeiträge zum Thema "Rationalisieren statt Rationieren" (Ersatzkassenforum 22.03.1994), z. B. Kaula K., Das Konzept der Ersatzkassen. Die Ersatzkasse 74 (4) 1994:134-137. 
kenkassen hingegen durchaus aufgeschlossen66. Mit dem 2. GKV-Neuordnungsgesetz (NOG) sind die Vertragspartner im ambulanten Bereich durch die geänderte Aufgabenstellung des Bundesausschusses Ärzte/Krankenkassen schließlich offiziell da$z u$ angehalten, den Leistungsrahmen von unnötigen und verzichtbaren (oder eklatant unwirtschaftlichen) Leistungen $\mathrm{zu}$ bereinigen (vgl. § 135 SGB V).

Damit scheint ein Tabu gebrochen; bereits die Annäherung an die Fragestellung birgt jedoch ausreichend Raum für Kontroversen. Auch die im folgenden gewählte Perspektive sucht einen kontroversen Standpunkt. Immer geht es um die Frage, ob und wie Solidarität in Zeiten knappen Geldes durch Anpassungen des Leistungsrahmens erhalten werden kann. Der hier gewählte Ansatz unterscheidet sich dabei von der juristischen Perspektive, welche nach einer definitorischen Bewältigung des Problems sucht' ${ }^{67}$, und von der politischen Perspektive, die sich durch den Aufruf an eine ethische Grundhaltung auszeichnet ${ }^{68}$. Beides ist wichtig, dürfte aber angesichts der Aufgabe per se nicht ausreichen. Aus der Perspektive der Ökonomie erscheint es vielmehr unerläßlich, daß ein insgesamt schlüssiger Ordnungsrahmen geschaffen wird, durch den das Handeln der Individuen so kanalisiert werden kann, daß die Ziele der Sozialpolitik im wesentlichen erreicht werden. Diese Forderung wird im folgenden erhoben und zugleich ein entsprechender Reformvorschlag zur Diskussion gestellt. Entgegen dem ersten Anschein besteht die Forderung aber nicht darin, eine Organisationsreform der GKV quasi vom Reißbrett aus durchzuführen, sondern darin, die wesentlichen Entwicklungslinien der GKV konstruktiv fortzusetzen.

66 Vgl. z. B. ein entsprechendes Faltblatt zur Versicherteninformation der BARMER Ersatzkasse, auf dessen Titelseite Bundespräsident Roman Herzog mit einem Ausschnitt aus seiner Berliner Rede am 26.04.1997 zitiert wird: „Wann endlich werden die Kosten der Arbeit von versicherungsfremden Leistungen befreit? ... Die Bürger erwarten, daß jetzt gehandelt wird“.

$67 \mathrm{Vgl}$. den Beitrag von Alexander Ehlers in diesem Band.

$68 \mathrm{Vgl}$. den Beitrag von Bernd Schirmer in diesem Band. 


\section{Politische Ausgangslage: Umbau der GKV-Reformbedürftigkeit}

\section{1 „Ausgabenbegrenzung“ als politische Maxime}

Seit rund zwei Jahrzehnten dreht sich die Gesundheitspolitik um die Frage, wie die Rate des Ausgabenanstiegs im Gesundheitswesen an die Entwicklung der Finanzierungsbasis angepaßt werden kann. Dabei legt ein Blick über die Grenzen nahe, daß dies kein typisch deutsches Problem ist. In nahezu allen OECDStaaten läßt sich das Phänomen steigender Gesundheitsquoten beobachten, das darauf hindeutet, daß die Ausgaben für medizinische Versorgung im Zeitablauf die jeweilige Einkommensentwicklung übersteigen. Hinter dem Bemühen, die Ausgabenentwicklung im Gesundheitswesen zu bremsen, steht, wie das Beispiel USA zeigt, nahezu unabhängig von der Art der Finanzierungsweise die Ansicht, daß die Ausgaben für medizinische Versorgung vor allem als ein volkswirtschaftlicher Kostenfaktor anzusehen seien. Dabei erscheint die Kostendämpfungspolitik in der Bundesrepublik im internationalen Vergleich durchaus erfolgreich. Als Erfolgsindikator gilt hier die über 15 Jahre nahezu konstante Gesundheitsquote Deutschlands. Vor allem die Ausgabenentwicklung in der GKV konnte durch zahlreiche gesetzgeberische Interventionen im wesentlichen im Rahmen des gesamtwirtschaftlichen Einkommenszuwachses gehalten werden (Abb. 1)69.

69 Vgl. Wasem J., Die Ursachen der Ausgabenanstiege in der medizinischen Versorgung. In: Arnold M, Lauterbach KW, Preuß KJ. Managed Care. Stuttgart 1997:75-91 
Abb. 1

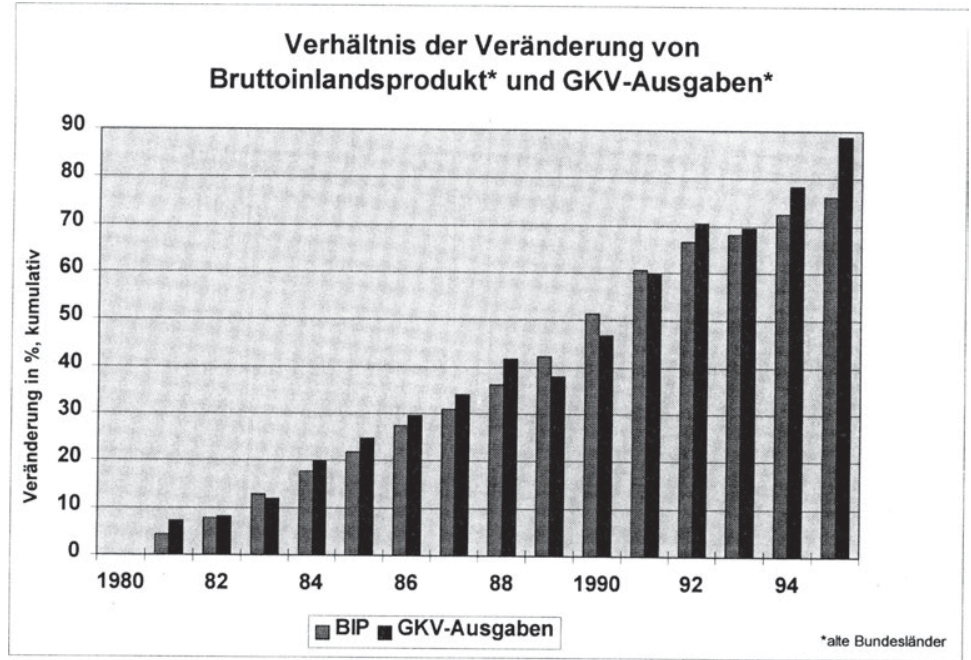

Quelle: BMG, Daten des Gesundheitswesen 1997

Innerhalb Deutschlands gilt die bisherige Kostendämpfungspolitik jedoch als weitgehend gescheitert, da sie das selbstgesetzte Ziel der Beitragssatzstabilität, abgesehen von kurzen Perioden unmittelbar nach einem Reformgesetz, im Zeitablauf nicht erreichen konnte. Aufgrund der Umlagefinanzierung der GKV sind „Ausgabenexplosion“ und „Einnahmenerosion" dabei zwei Seiten der gleichen Medaille. Bis zum GSG befand sich vor allem die Ausgabenentwicklung im Visier der Politiker, während nunmehr zusätzlich die Erosion der Finanzierungsgrundlagen in der Sozialversicherung die Politik in absehbarer Zeit erneut zum Handeln zwingt70.

Im Kern der deutschen Problematik besteht folglich die historisch bedingte Festlegung der Beitragsbemessung auf die Einkommen aus abhängiger Beschäftigung. Vor dem Hintergrund einer seit Jahren sinkenden Lohnquote müssen gegenwärtig deshalb akute Einschnitte in die Finanzgrundlage durch hohe Arbeitslosigkeit und durch die Zunahme sozialversicherungsfreier Beschäftigungsverhältnisse bzw. der Umwandlung vieler Arbeitsverhältnisse in (Schein-)Selbständigkeit verkraftet werden, die bei einer Einbeziehung anderer Einkunftsarten weit weniger deutlich ausfal-

70 „Seehofer: Soziales vom Lohn lösen“, Handelsblatt Nr. 228 vom 26.11.1997, S. 5 
len würden. Zweifel an der dauerhaften Tragfähigkeit der bestehenden Finanzierungsgrundlage erhärten sich mit Blick auf den sich eher noch verschärfenden internationalen Standortwettbewerb und den dadurch beschleunigten Strukturwandel der Beschäftigungsverhältnisse sowie im Hinblick auf den langfristig steigenden Altenquotienten und die damit verbundenen einnahmen- und ausgabenseitigen Belastungen.

Im Kontext des Standortwettbewerbs und zur Eindämmung der Belastungen des sozialen Sicherungssystems durch die Arbeitslosigkeit fokussiert sich das politische Interesse auf eine Entlastung der Lohnnebenkosten: Parteiübergreifend herrscht Einigkeit über die Notwendigkeit einer Rückführung oder zumindest einer Stabilisierung der Arbeitgeberbeiträge zur Sozialversicherung.

Mit der Maxime „Beitragssatzstabilität" steckt die Gesundheitspolitik jeder Partei jedoch in einem Dilemma: Während die einen sich eher für die Interessen der Leistungsanbieter einsetzen und alle Maßnahmen verhindern, die zu einem Abbau von Angebotskapazitäten und mithin zu einem Einbruch der Wachstumsdynamik im Gesundheitswesen führen könnten, sorgen sich die anderen um den Erhalt der bisher breiten Zugangsrechte und widersetzen sich allen Maßnahmen, die zu einer Reduktion des Leistungskataloges oder einer stärkeren finanziellen Eigenbeteiligung der Patienten führen. Als kleinster gemeinsamer Nenner dürfte so ein Versuch, drängende strukturelle Reformen durch eine partielle Erweiterung der Finanzierungsbasis, z. B. durch eine Anhebung der Beitragsbemessungsgrenze oder eine Finanzierung sogenannter versicherungsfremder Leistungen aus dem allgemeinen Steueraufkommen, am meisten politische Unterstützung erfahren.

Ein Konzept für eine ordnungspolitische Neugestaltung der GKV, das erlauben würde, die Zielkonflikte in der aktuellen Gesundheitspolitik oder in den gesundheitspolitischen Zielvorstellungen der Opposition aufzulösen, steht damit jedoch weiterhin aus. Gleichwohl vollzieht sich bereits eher unbemerkt ein Wandel, da einige der in den letzten Jahren in Kraft getretenen Neuregelungen einen tiefgreifenden und nachhaltigen Umbruch in der GKV eingeleitet haben. 


\subsection{Tiefgreifende Veränderung des Wettbewerbs der Krankenkassen}

Seit 1989 wurden historisch bedingte Unterschiede in den Wettbewerbsvoraussetzungen einzelner Kassenarten durch eine Reihe gesetzgeberischer Eingriffe schrittweise aufgehoben. Durch Angleichungen im Vertrags- und Mitgliedschaftsrecht hat sich die Bedeutung des gegliederten Systems der Krankenversicherung gravierend verändert. Weitreichende Wirkung geht insbesondere von der Einführung des Risikostrukturausgleichs (1994) und der allgemeinen Kassenwahlfreiheit (1996) aus ${ }^{71}$.

Durch den Risikostrukturausgleich (RSA) verlieren traditionelle Wettbewerbsvor- oder -nachteile, vor allem das Lohnniveau der Versichertengemeinschaft, aber auch deren Alters- oder Mitversichertenstruktur, an Bedeutung. Unter RSA-Bedingungen lassen sich Wettbewerbsvorteile künftig nur noch durch eine unterdurchschnittliche altersspezifische Ausgabenstruktur erreichen.

Die Beseitigung der historischen, berufsbezogenen Mitgliedschaftsregelungen bedingt eine Öffnung des Wettbewerbs aller gesetzlichen Krankenkassen untereinander. Die Kassen verlieren dadurch nicht nur ihre angestammten Identitäten, z. B. als Arbeiter- oder Angestelltenkrankenkasse. Auch die Bedeutung externer Wandlungsprozesse nimmt ab, so z. B. die stetige Zunahme von Arbeitsverhältnissen mit Angestelltenstatus, als ein die Wettbewerbschancen einzelner Kassen maßgeblich beeinflussender Faktor. Das eigenständige Handeln der Wettbewerber gewinnt dagegen an Bedeutung. In den Augen der Versicherten dürfte künftig vor allem das Preis-Leistungs-Verhältnis der Krankenkassen zählen. Aktive Preispolitik oder bewußte Gestaltung ihres Leistungsangebots über das Niveau kleinerer Werbegags hinaus lag den Krankenkassen jedoch bislang fern. Die Kombination aus RSA und allgemeiner Wahlfreiheit zwingt die meisten Kassen nun, sich vom Selbstverständnis eines Gesetze ausführenden Organs der mittelbaren Staatsverwaltung zu lösen und ein neues, marktorientiertes Unternehmensprofil zu entwickeln ${ }^{72}$.

$71 \mathrm{Zu}$ den maßgeblichen Änderungen im Vertragsrecht zählen u. a. die Ausdehnung von Kontrahierungszwang und Sicherstellungsauftrag der KVen auch auf Ersatzkassenversicherte (1989) sowie die Vereinheitlichung der Vertragspartnerschaften im Sinne einer „Regionalisierung" auf Länderebene (1993).

72 Vgl. z. B. Beitrag von Gerd Glaeske in diesem Band. 
Mit Blick auf die zunehmende Preisempfindlichkeit der Versicherten und auf die Wirkung des RSA sind die Krankenkassen vor allem gefordert, die Ausgabenentstehung in ihrem Sinn zu beeinflussen. Um dieses Interesse zusätzlich zu fördern und die Anstrengungen der Krankenkassen zu beschleunigen, sah der Gesetzgeber im 2. NOG (1997) vor, daß Beitragssatzanhebungen einer Kasse durch eine proportionale Anhebung der Zuzahlungen ihrer Versicherten sanktioniert würden. Hier wird eine Instrumentalisierung des Wettbewerbs für die Ziele der Kostendämpfungspolitik offenkundig.

Angesichts dieser hochgespannten Erwartung erscheint das 2. NOG jedoch inkonsequent, denn der Gesetzgeber gibt den Krankenkassen keine nennenswerten Instrumente zur Realisierung dessen an die Hand. Obgleich es die einzelnen Kassen sind, die sich den Versicherten gegenüber mit einem günstigen Beitragssatz und/oder einer spezifischen Versorgungsqualität profilieren müssen, wird der Großteil des Ausgabenvolumens weiterhin von korporativen Verhandlungen auf der Ebene der Verbände bestimmt. Alle vertragsrechtlichen Reformüberlegungen sind zudem dort sehr zurückhaltend ausgefallen, wo das korporative Kräftegleichgewicht zuungunsten der Leistungsanbieterinteressen gefährdet worden wäre. Es bleibt daher offen, welches Potential die neuen Vertragsmöglichkeiten des $\S 73$ a SGB V (Strukturverträge) und die Neuregelung des $\S 63 \mathrm{ff}$. SGB $\vee$ (Modellvorhaben) wirklich entfalten können.

Schon jetzt wird jedoch erkennbar, daß eine Ausrichtung des Krankenkassenwettbewerbs allein auf Kostensenkungsziele zu kurz greift. Soweit sie überhaupt Erfolg hat, dürfte eine rigorose Sparpolitik mittelfristig vielfach kontraproduktiv wirken, wenn Ausgaben jeweils sektoral minimiert werden, ohne sektorübergreifende Substitutionspotentiale zu erschließen. Eine sektorübergreifende Steuerung der Ausgabenentwicklung erfordert jedoch eine Orientierung an typischen Krankheitsverläufen und übergreifenden Therapiezielen und setzt insoweit auch auf Kostenträgerseite die Bereitschaft zur inhaltlichen Mitgestaltung von Versorgungsabläufen voraus. Durch ein solches Handeln übernimmt der Kostenträger unweigerlich auch Mitverantwortung für medizinische Handlungsabläufe. Soweit man diesbezüglich aus dem Beispiel amerikanischer Managed-Care-Organisationen lernen kann, wird ein erfolgreicher Kostensenkungswettbewerb daher auch einen Qualitätswettbewerb nach sich ziehen. 


\section{3 „Neudefinition der Solidarität“}

Die bisherige Kostendämpfungspolitik im Gesundheitswesen operierte insbesondere während der Phase der relativ strikten Ausgabendeckelung zwischen 1993 und 1996 mit der Aussage, daß Rationierung auch unter den Bedingungen der Budgetierung aufgrund der ausreichend vorhandenen Rationalisierungsreserven „im System“ vermeidbar sei. Im Lauf des Gesetzgebungsprozesses zum 2. NOG fand in diesem Punkt ein bemerkenswerter Überzeugungswandel statt. Nunmehr rechtfertigt die Aussage, die Rationalisierungsreserven seien weitgehend ausgeschöpft, die Forderung, mehr Geld müsse „ins System“.

Die Kostendämpfungspolitik gibt sich somit selbst geschlagen; statt dessen wird nunmehr umgesetzt, was von seiten des Bundesgesundheitsministers bereits im Jahr 1993, nach Verabschiedung des GSG, womöglich in Vorwegnahme eines möglichen Scheiterns bzw. in Erwartung des üblichen Wiederansteigens der Ausgabenentwicklung nach einem gesetzgeberischen Einschnitt, angekündigt worden war: eine "Neudefinition der Solidarität".

Dabei ist bisher nicht beabsichtigt, den Solidarbegriff, wie inn das Sozialgesetzbuch im Grundsatz definiert, zu verändern, wohl aber dessen praktische Bedeutung für die Versicherten. Dies geschieht auf vielfältige Weise: durch höhere Zuzahlungen der Versicherten oder die fakultative Durchbrechung des Sachleistungsprinzips, durch Bonusregelungen für Nichtinanspruchnahme von Leistungen oder die Kostenerstattungsoption. Hinzu kommen Leistungsausgrenzungen in nennenswertem Umfang, wie z. B. beim Zahnersatz. Alle diese Maßnahmen belasten unmittelbar die Patienten - unter Umgehung der Solidargemeinschaft ${ }^{73}$. Sie sind Ergebnis einer Suche nach neuen Finanzierungsquellen, die ohne jede Belastung der Arbeitgeberbeiträge erschlossen werden können. Politisch wird hierfür der Euphemismus größerer Eigenverantwortung bemüht.

${ }^{73}$ So wird die Zunahme der direkten Belastung der Patienten aufgrund der um 5 DM erhöhten Zuzahlungen für Arzneimittel auf ein Volumen von rund 3,4 Mrd. DM geschätzt. Dies entspricht einem Anstieg um $90 \%$. Vgl. z. B. eine Hochrechnung des Wissenschaftlichen Instituts der Ortskrankenkassen: Litsch M., Schröder H., Zuzahlungserhöhung um weitere 5,-- DM: Auswirkungen im Arzneimittelbereich. Sozialer Fortschritt (5) 1996:122-125. 
Der aktuelle Trend zur direkten Belastung der Patienten widerspricht nicht nur dem gängigen Grundverständnis von Solidarität, sondern stellt auch eine radikale Abkehr von der bisherigen Entwicklungslinie der GKV dar: Nach der geltenden Definition des Sozialgesetzbuches steht Solidarität für die einkommensabhängige Finanzierung und die beitragsunabhängige, bedarfsgerechte Inanspruchnahme von medizinischer Versorgung. Angesichts der umfassend definierten Aufgabenstellung der Krankenkassen, nämlich Sachleistungen zur Heilung und Linderung von Krankheiten sowie zum Erhalt von Gesundheit bereitzustellen (vgl. §§ 1, 2, 11, 27 SGB V), wird die redistributive Funktion der einkommensabhängigen Finanzierung unmittelbar deutlich. Sie steht erklärtermaßen im Widerspruch zum Prinzip der Risikoäquivalenz privatwirtschaftlicher Versicherungen, denn der Grundsatz gleicher Zugangsrechte läßt sich ohne ein gewisses Maß der Einkommensumverteilung nicht realisieren. Die direkte Beteiligung der Patienten verzichtet demgegenüber sogar auf jeglichen, auch den versicherungstechnischen Risikoausgleich und führt damit das Äquivalenzprinzip zum Extrem.

Abb. 2

Das Solidarprinzip gestern, heute, morgen (?)

\begin{tabular}{|l|l|l|l|}
\hline \multirow{2}{*}{ Dimensionen } & \multicolumn{2}{|c|}{ Bedeutungswandel } & \multicolumn{1}{|c|}{ "Neudefinition } \\
\cline { 2 - 3 } der Solidarität“
\end{tabular}

Quelle: Eigene Darstellung 
Das Verständnis von Solidarität ist keine historische Konstante (Abbildung 2). An anderer Stelle ist bereits dargelegt worden, daß Solidarität und Eigenverantwortung keineswegs unvereinbare Grundsätze sind und daß eine sinnvolle Verknüpfung beider Prinzipien in der Frühphase der GKV durchaus schon einmal gelungen ist ${ }^{74}$. Im Gegensatz zu heute bestand die Funktion der einkommensabhängigen Finanzierung damals vor allem darin, eine versicherungstechnische Äquivalenz zwischen Beitrag und Leistung herzustellen. Dies gelang verhältnismäßig einfach, als die Leistung im wesentlichen in der Zahlung des ebenfalls lohnabhängigen Krankengeldes bestand. Dazu mußte allerdings gewährleistet sein, daß die Versichertengemeinschaft aus einigermaßen homogenen Risiken bestand und Sachleistungen nur nach strikter Prüfung der Bedürftigkeit gewährt wurden. Diese Bedingungen waren in den frühen Solidargemeinschaften weitgehend erfüllt; es galt folglich das Prinzip der Gruppenäquivalenz. Der Solidarbegriff stand dementsprechend vor allem für das Prinzip gegenseitiger Hilfe (Reziprozität) und setzte Eigenverantwortung zumindest implizit voraus.

Im Unterschied zum aktuellen Verständnis des Begriffes beschränkte sich Eigenverantwortlichkeit damals nicht auf eine bloBe Finanzierungsfunktion, sondern beinhaltete auch, daß die Mitglieder einer Versichertengemeinschaft im Rahmen der Selbstverwaltung auch über den Leistungsumfang, die Rigidität der Bedürttigkeitsprüfung bei der Leistungsgewährung und damit über die Höhe des Beitragssatzes mitzubestimmen hatten, wobei die aktive Einbindung der Versicherten nicht zuletzt aus sozialpädagogischen Überlegungen gewünscht wurde. Das Prinzip der Gruppenäquivalenz entfaltet somit auch eine rechtsphilosophische Bedeutung, nämlich als Einheit von Handlung und Haftung einer (überschaubaren) Solidargemeinschaft. Die damit einhergehenden Mechanismen der Selbstbegrenzung wurden jedoch im Zuge einer zunehmenden Vereinheitlichung des Leistungs- und Vertragsrechts schrittweise demontiert und insbesondere seit dem 2. Weltkrieg durch eine an Umverteilungszielen orientierte Sozialpolitik ersetzt.

$74 \mathrm{Vgl}$. Stillfried D. Gesundheitssysteme im Wandel. Das Dilemma zwischen Bedarfskonzept und Eigenverantwortung: medizinische Grundsicherung als Reformperspektive? Bayreuth 1996: Abschnitt 3.2.2. 
Die im Ansatz radikale Abkehr von der distributiven Ausrichtung des heutigen Solidarverständnisses durch eine Verlagerung gröBerer Finanzierungsanteile auf die unmittelbar Betroffenen, ohne Zwischenschaltung eines irgendwie gearteten Mechanismus zur Risikostreuung, wirkt jedoch konzeptlos und erscheint als Reformrezept angesichts der bevorstehenden Aufgabe nicht zukunftsfähig. Es ist kaum glaubwürdig, auf diesem Weg die sich abzeichnenden Finanzierungsdefizite der GKV aufzufangen, zugleich aber das „System" der GKV und ihre korporativen Interessenlagen weiterhin im wesentlichen unangetastet zu lassen und zudem den bisher hohen sozialpolitischen Anspruch aufrechterhalten zu wollen. Es fehlt ein klares Bekenntnis zum künftigen Inhalt und Organisationsprinzip der solidarischen Krankenversicherung.

\section{Neuordnung des Leistungskatalogs als Reformschritt}

\subsection{Ziel: Solidarität erhalten}

Die Frage der Neuordnung des Leistungskatalogs wird unter der Perspektive ins Feld geführt, Elementares von Verzichtbarem zu trennen, um die (gewohnte) Solidarität im Kern erhalten zu können.

Wie im folgenden gezeigt wird, läßt sich diese scheinbar randständige Korrektur jedoch nicht ohne ein systematisches Konzept des künftig gewünschten Solidarbegriffs und seiner Bedeutung für die Versicherten durchführen. Eine Begrenzung des Reformansatzes auf die Anpassung des Leistungsrahmens greift auch zu kurz. Jeder Entwurf einer Problemlösung muß vielmehr alle Faktoren berücksichtigen, die den Geltungsbereich des Solidarprinzips prinzipiell bestimmen. Er muß zudem die Wechselwirkungen dieser Faktoren aufeinander beachten, aus denen letztlich der faktische Geltungsbereich resultiert.

\subsection{Geltungsbereich des Solidarprinzips}

Wie in jeder nach dem Umlageprinzip finanzierten Absicherungsform ergibt sich der Geltungsbereich des Solidarprinzips auch in der GKV aus folgenden vier Faktoren:

- dem Kreis der Leistungsberechtigten

- dem Leistungsrahmen 
- dem in der Leistungsgewährung verwendeten Notwendigkeitsbegriff und

- dem Ausgabenvolumen

Historisch gesehen wurde das heutige Absicherungsniveau in der GKV durch eine immer weiter greifende gesetzliche Gestaltung der vier Faktoren in etwa der genannten Reihenfolge, gewissermaßen in mehreren Durchgängen, nach oben geschraubt:

Der Kreis der Leistungsberechtigten wurde von anfänglich weniger als $10 \%$ der Bevölkerung durch die sukzessive Einbindung verschiedener Berufsgruppen, der Familienmitglieder der Versicherungsnehmer, der Rentner, Studenten, Künstler etc. auf heute rund $90 \%$ der Bevölkerung ausgedehnt.

Der Leistungsrahmen wurde stetig erweitert, jedoch nur selten, wie etwa im GRG 1989, auch z. T. gekürzt. Entscheidend ist hierbei jedoch weniger die Summe einzelner Ergänzungen, sondern der grundsätzliche Wandel der Aufgabenstellung der GKV, in der medizinische Sachleistungen ursprünglich nahezu ausschließlich der beschleunigten Wiederherstellung der Arbeitsfähigkeit (und damit der Verkürzung der Krankengeldzahlung) dienten, hin zum nunmehr seit 1989 gesetzlich gefaßten Auftrag, „die Gesundheit der Versicherten zu erhalten, wiederherzustellen oder ihren Gesundheitszustand zu bessern" ( $§ 1 \mathrm{SGB} V$ ), d. h. Sorge für die Gesundheit im allgemeinen zu tragen.

Im Zuge dieser Richtungsänderung hat sich zweifelsohne auch die Auslegung des medizinischen Notwendigkeitsbegriffes in der GKV gewandelt. Wurden die Beschwerden eines Patienten früher strikt im Hinblick auf deren ursächlichen Zusammenhang mit der Beschäftigung und deren konkrete Beeinträchtigung der Arbeitsfähigkeit beurteilt, so haben die Versicherten heute Anspruch auf eine Behandlung, die sich ausschließlich an dem orientiert, was die Medizin im Hinblick auf die vorliegende Erkrankung an bewährten oder hinreichend erforschten Therapiemöglichkeiten bereithält. Das ansteigende Ausgabenniveau der GKV wurde über lange Zeit als Resultante akzeptiert, wenn auch begleitet von Korrekturen im Hinblick auf die im Zusammenhang mit dem Leistungsrahmen geltenden Vergütungsformen.

Soweit erscheint der Geltungsbereich des Solidarprinzips im Wege regulierender Gesetzgebung durchaus zielkonform definierbar. 
Die komplexe Wechselwirkung der vier Faktoren erschließt sich jedoch erst, wenn von seiten der Politik versucht wird, alle vier Dimensionen gleichzeitig zu steuern. Dies wird in Abbildung 3 schematisch dargestellt.

Abb. 3

\begin{tabular}{|c|}
\hline $\begin{array}{c}\text { Geltungsbereich des Solidarprinzips: } \\
\text { Aktionsparameter, Ergebnisparameter und Verflechtung der sozialpolitischen } \\
\text { medizinischen und ökonomischen Perspektive }\end{array}$ \\
\hline
\end{tabular}

\begin{tabular}{|c|c|c|c|}
\hline $\begin{array}{c}\text { ex ante definiert: } \\
\text { Leistungsrahmen } \\
\text { abgesicherte Risiken } \\
\text { (inkl. Vergütungsregelung) }\end{array}$ \\
\hline
\end{tabular}

Quelle: Eigene Darstellung

In den vier interdependenten Feldern sind die genannten Faktoren einerseits als Aktionsparameter der Sozialpolitik (oben im Feld), andererseits als Ergebnisparameter (in kursiver Schrift unten im Feld) dargestellt. Während die Aktionsparameter von der Politik zur Einflußnahme auf die Reichweite des Solidarprinzips beabsichtigt verändert werden können, stellen sich die Ausprägungen der Ergebnisparameter $u$. U. als unbeabsichtigtes $\mathrm{Er}$ gebnis der Wechselwirkungen zwischen den Feldern ein. Ihre Gestaltungsabsicht kann die Politik selbst nur in Grenzen umsetzen, denn per Gesetz können nur die institutionellen Rahmenbedingungen für das Verhalten der Wirtschaftssubjekte im Gesundheitssystem abgesteckt werden.

Problematisch ist dabei insbesondere die Querachse, d. h. die Wechselwirkung zwischen dem unbestimmten Rechtsbegriff des Notwendigen und der Bedeutung seiner Auslegung für den Umfang der konkret abgesicherten Risiken. Beides korrespondiert eng mit der Definition des Leistungskataloges. Das sozialpolitische Interesse daran, welche Risiken im einzelnen abgesichert werden sollten, kommt in der Formulierung des Leistungskatalogs zum Ausdruck. Abgesehen von Ausnahmen mit z. T. enorm detaillierten Regelungen (vgl. z. B. $\S 30$ SGB in der Fassung 
d. 2 NOG) präsentiert der gesetzliche Leistungskatalog aber eher grobe Umrisse, wie z. B. „die Versicherten haben Anspruch auf Krankenbehandlung ... [diese] umfaßt ärztliche Behandlung ... Krankenhausbehandlung ..." (§ 27 SGB V), die z. B. durch die Richtlinien des Bundesausschusses Ärzte/Krankenkassen oder durch Satzungsregelungen der Krankenkassen nähere Bestimmung erfahren. Zur Konkretisierung des Leistungsanspruchs bzw. zur Abrechnung einer Leistung zu Lasten der GKV kommt es jedoch nur nach einer Prüfung, ob die konkrete gesundheitliche Situation eines Patienten im einzelnen eine Leistung rechtfertigt, welche sich unter eine Anspruchsdefinition bzw. einen Abrechnungstatbestand der GKV subsumieren läßt. Diese Prüfung liegt jedoch weitestgehend in den Händen der Leistungsanbieter, deren Handeln u. a. auch der ökonomischen Anreizwirkung des jeweiligen Vergütungssystems unterliegt ${ }^{75}$.

Welcher Ressourcenintensität es bedarf, um einen bestimmten Personenkreis ausreichend gegen ein Krankheitsrisiko in wünschenswertem Umfang abzusichern, ergibt sich somit im wesentlichen aus der Summe der individuellen Handlungsweisen auf Leistungserbringerseite im Umgang mit den an sie herangetragenen Anfragen und Affektionen. Die Ressourcenintensität der Versorgung wird somit entscheidend durch die Indikationsstellungen und deren Ausführungsmodalitäten im Behandlungsprozeß bestimmt.

Das solidarisch abzusichernde Krankheitsrisiko bezieht sich auf die finanzielle Implikation derjenigen medizinischen Versorgungsprozesse, die bei einer gegebenen gesundheitlichen Beeinträchtigung als unerläßlich angesehen werden. Das Risikopotential steigt folglich in Abhängigkeit vom Ressourcenverbrauch der Medizin, sei es aus Gründen ineffizienter Organisation oder aufgrund der steten Weiterentwicklung der medizinischen Handlungsmöglichkeiten.

Da historisch gesehen vor allem die drei Faktoren Definition des Personenkreises, Definition des Leistungsrahmens und ein (zunehmend erweiterter) Notwendigkeitsbegriff die Wertigkeit des Solidarprinzips bestimmt haben, mußte der vierte Faktor, nämlich

75 Fiedler E. Optimale Ressourcennutzung im Spannungsfeld von ambulanter und stationärer Versorgung. KBV-Kontext Heft 5, 1997:15-26 
das zur Verfügung gestellte Ressourcenvolumen, in der Regel als variables Residuum angesehen werden.

Wird nun aber vor dem Hintergrund der Erfahrung, daß die Anforderungen an die Ressourcenverfügbarkeit keineswegs ex ante definierbar, sondern im wesentlichen Ergebnis des Verhaltens der Leistungsanbieter sind, das Ausgabenvolumen der GKV administrativ begrenzt, so wird schließlich der Umfang der abgesicherten Risiken zum variablen Residuum. Soweit einzelwirtschaftliche Rationalität das Verhalten der Leistungsanbieter bestimmt, darf aufgrund einer Ausgabenbudgetierung keine grundsätzliche Verhaltensänderung auf der Leistungserbringerseite erwartet werden, allenfalls eine Richtungsänderung prinzipiell unveränderter Verhaltensmuster: Anstatt bei gegebenem Honorar ihre Menge anzupassen, werden sie nun die Menge oder Qualität einem gegebenem Ressourcenvolumen anpassen.

Die Ursachen der bisherigen Ausgabensteigerungen, nämlich die Erwartungen und die aufgrund des medizinisch-technischen Fortschritts sogar steigenden Ansprüche der Solidargemeinschaft in bezug auf die Zugänglichkeit eines prinzipiell zugesagten Leistungsrahmens, wirken somit unverändert weiter. Diese Spannung wird folglich auf das Feld der Indikationsstellung verlagert. Dort muß das Problem konkurrierender Anrechte der Mitglieder der Solidargemeinschaft auf Zuwendungen aus dem gemeinsamen Ressourcenvolumen gelöst werden. Die Bewältigung dieser Frage entscheidet über die faktische Tragweite und die praktische Bedeutung des Solidarprinzips.

Dabei besteht das Problem in der Spannung, die der unbestimmte Rechtsbegriff der (medizinischen) Notwendigkeit erzeugt. So ist z. B. der gedankliche Bezugspunkt bei Arzthaftungsfragen ein von der ökonomischen Realität abstrahierter Behandlungsverlauf nach dem Stand wissenschaftlicher Erkenntnis. Hierbei handelt es sich, wie in der Erwartung der Versicherten, um das Maximalprinzip des Notwendigen. Andererseits ist Notwendigkeit keine naturgesetzliche Konstante, sondern ergibt sich aus einem breiten Spektrum unterschiedlicher Interpretationsmöglichkeiten und Verhaltensweisen, dessen „allgemein anerkannter" Kern bei Streitigkeiten jeweils im einzelnen von neuem normativ bestimmt werden muß. Die situative Bedingtheit der Notwendigkeit hat im Alltag der Versorgung zur Folge, daß unter restriktiven ökonomischen Bedingungen der Bezugspunkt medizinischer Notwendig- 
keit bei gleichbleibendem Leistungsrahmen im praktischen Handeln der Ärzte schleichend nach unten korrigiert wird. Für den einzelnen heißt dies, daß der im Leistungsrahmen definierte Anspruch im Extremfall nur noch mit einer groben Wahrscheinlichkeit konkretisierbar ist. Das gleichermaßen krasse wie bekannte Beispiel hierfür dürfte der Zugang zur Dialysebehandlung in Großbritannien sein, der einigen Patientengruppen trotz anderslautender Definition des Leistungsrahmens de facto nicht mehr offensteht.

Eine Politik, die gleichermaßen bemüht ist, das sozialpolitisch akzeptable Ausgabenvolumen administrativ vorzugeben und gleichzeitig elementare Risiken solidarisch abgesichert zu wissen, müßte folglich den Leistungsrahmen in idealer Weise so anpassen, daß die verfügbaren Ressourcen optimal ausgenutzt werden. Dies würde jedoch Kenntnis über mindestens drei Dinge voraussetzen: Zum einen wäre eine Einigung darüber erforderlich, was elementare Risiken sind und wie diese in pragmatischer Weise von Verzichtbarem abgegrenzt werden können. Zum anderen müßte das Verhalten der Leistungsanbieter als Reaktion auf die konkrete Anreizstruktur im Hinblick auf das Behandlungsergebnis prognostizierbar sein. Eine Erfolgsbeurteilung der Korrektur des Leistungsrahmens kann schließlich nicht erfolgen, ohne die Risikostruktur im Versichertenklientel zu kennen. Es deutet sich an, wie grob das Instrument einer Anpassung des Leistungsrahmens im Hinblick auf die von der Politik selbst gewählte Zielsetzung ist. Damit stellt sich auch die Frage, ob und inwieweit die Politik überhaupt die geeignete Handlungsebene zur Lösung der Frage ist.

\subsection{Geltungsbereich der Solidarität - Gestaltungsoptionen}

Ausgehend von der Charakterisierung des Solidarprinzips im SGB V (einkommensabhängige Beiträge, beitragsunabhängige Leistungsinanspruchnahme) lassen sich die Gestaltungsoptionen zu seiner Neudefinition in Fragen der Finanzierung und der $\mathrm{Zu}$ gänglichkeit von Leistungen gliedern (Abbildung 4). 
Abb. 4

\section{Reichweite der Solidarität - Dimensionen und Gestaltungsmöglichkeiten}

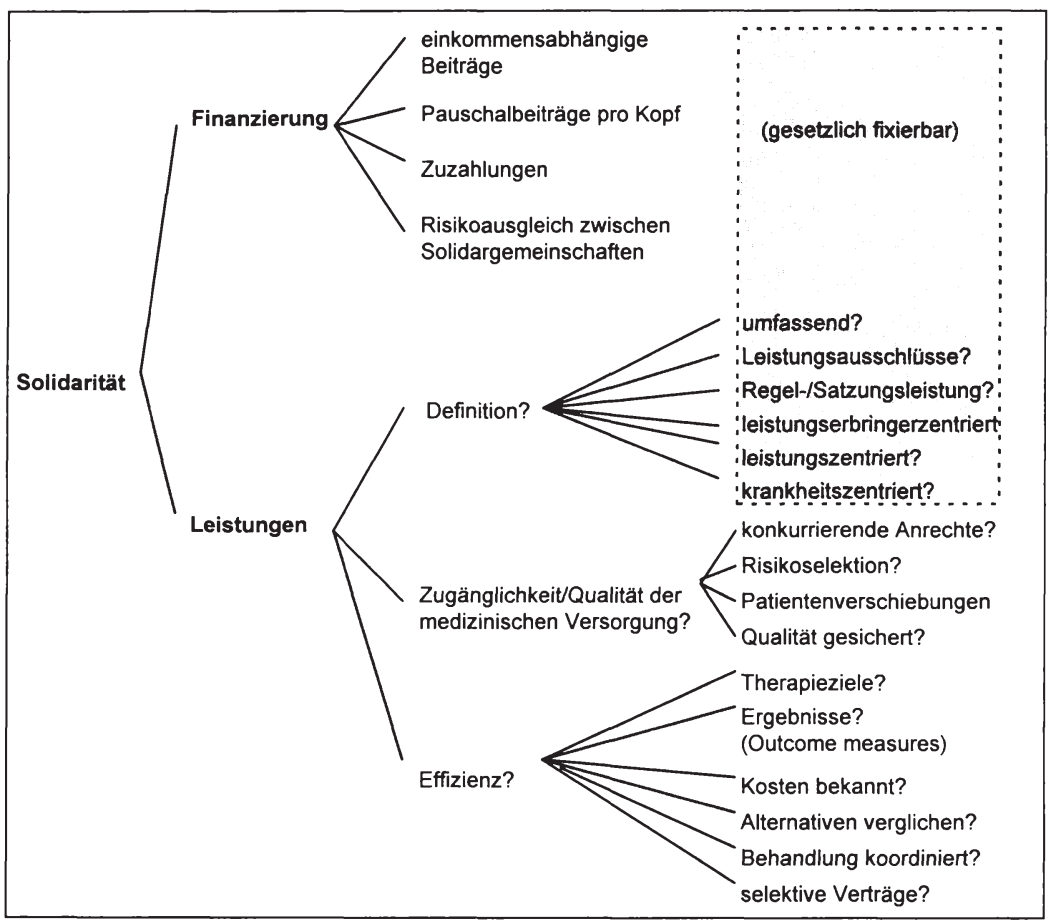

Quelle: eigene Darstellung

Einer eindeutigen gesetzlichen Regelung erschließt sich dabei insbesondere der farbig unterlegte Bereich in Abb. 4. Dieser umfaßt neben Optionen im Bereich der Finanzierungsmodalitäten die unterschiedlichen Möglichkeiten, den Leistungsrahmen zu beschreiben.

Geht man davon aus, daß die Steigerungsrate der Ausgaben im Zeitablauf im wesentlichen aus der Anwendung eines gegebenen Leistungsrahmens entsteht, so kann - dies ist der Kern meines Arguments - das Problem steigender Ausgaben auf Dauer nicht allein dadurch gelöst werden, daß der Leistungsrahmen gekürzt oder punktuell angepaßt wird.

Die Krise der GKV in ihrer gegenwärtigen Form besteht in der immer deutlicher empfundenen Bedrohung der faktischen Reich- 
weite der Solidarität. Folgt man dieser Ansicht, so besteht die sozialpolitische Herausforderung in bezug auf die Neudefinition der Solidarität vor allem darin, ein Verfahren zu bestimmen, um das höchste faktisch erreichbare Absicherungsniveau auszuloten, das bei einem im Hinblick auf die Finanzierungslast verträglichen Ausgabenniveau realisiert werden kann. Die Frage, wie der Leistungsrahmen zu beschreiben ist, rückt damit im Vergleich zu den Fragen, wie diese Beschreibung im einzelnen umzusetzen ist (vgl. Rubriken Zugänglichkeit, Qualität und Effizienz der Leistungsgewährung in Abb. 4) in den Hintergrund. Die Fragen der Umsetzung können unmittelbar jedoch nur von den Vertragspartnern angegangen und ausgefüllt werden.

Zu Recht wird in diesem Zusammenhang gerade von seiten der Vertragsärzteschaft darauf hingewiesen, daß eine Neugestaltung des Leistungskataloges ohne flankierende Maßnahmen zur Gewährleistung strengerer Indikationsstellungen das Ziel einer Konzentrierung des Leistungsgeschehens auf das Wesentliche und Notwendige verfehlen muß76.

Bei den Fragen der Umsetzung des Leistungskataloges steht folglich die Einigung über Wertigkeiten, die in die Konkretisierung des medizinischen Notwendigkeitsbegriffs eingehen, im Vordergrund. Es geht darum, Formen der Steuerung des konkreten Leistungsgeschehens zu entwickeln, in der sich Leistungsnachfrager und Leistungsanbieter zunehmend genauer darüber einigen, nach welchen Kriterien der Begriff der medizinischen Notwendigkeit im Einzelfall, $d . h$. bei einer konkreten Indikationsstellung, auszulegen ist, damit dieser somit auch für die Versicherten als "Begünstigte" ohne Feststellung über eine Klage verläßlich einschätzbar wird.

Eine Neudefinition der Solidarität, die von der Interpretationsbedürftigkeit medizinischer Notwendigkeit ausgeht, muß jedoch auch die Bedeutung des patientenseitigen Verhaltens berücksichtigen und Wertungen der Patienten bewußt mit einbeziehen, welche die Auslegung medizinischer Notwendigkeit im konkreten Einzelfall beabsichtigt oder unbeabsichtigt beeinflussen. Da die Versicherten und Patienten als Nachfrager das Leistungsgesche-

76 Schorre W. Neuordnung des Leistungskataloges in der Gesetzlichen Krankenversicherung. Einführungsreferat zum III. Symposium der Kassenärztlichen Bundesvereinigung, 2./21.10.1997 (Manuskript) 
hen mitgestalten, darf deren Rolle in einem System, das den Wettbewerb auf der Anbieterseite betont, ordnungspolitisch nicht ignoriert werden. Um eine schlüssige Antwort auf die aufgeworfenen Fragen zu liefern, muß die Neudefinition der Solidarität - so scheint es - folglich in einer weitgreifenden Rekonstruktion der solidarischen Absicherungsform gesucht werden.

\section{Neudefinition der Solidarität als Reformschritt}

\subsection{Entwicklungslinien konstruktiv fortsetzen}

Wie könnte eine entsprechende Weiterentwicklung der GKV zur langfristigen Sicherung der Solidarität theoretisch aussehen? Gesucht ist ein theoretisches Konzept von hoher politischer Integrationskraft, dessen Basis der zugrunde gelegte Solidarbegriff ist. Da das Regelungsgefüge der GKV in seiner aktuellen Form nicht aus einem schlüssigen theoretischen Konzept abgeleitet worden, sondern im historischen Entwicklungsprozeß langsam als ein Amalgamat von Regelungsbestandteilen angewachsen ist, deren Begründung und Zielsetzung sich in den verschiedenen Entwicklungsschritten auf ein durchaus unterschiedliches Verständnis von Solidarität bezog, müssen aus dem, was uns heute als GKV gegenübersteht, zunächst der Solidarbegriff und die dazu passenden Organisationsprinzipien "herauspräpariert" werden, die einem Konzept zur künftigen Ausgestaltung der Rahmenbedingungen zugrunde gelegt werden sollen. Besonderes Augenmerk kommt dabei den drei Teilaspekten Finanzierung, Kassenwettbewerb und Eigenverantwortung zu, an denen sich die Krise der GKV (vgl. die Ausführungen zur politischen Ausgangslage) manifestiert.

\subsubsection{Finanzierung nach dem Leistungsfähigkeitsprinzip}

Die Funktion der einkommensabhängigen Beitragsschöpfung besteht nach heutigem Verständnis darin, die nötige Umverteilung von Einkommen sicherzustellen, damit allen Versicherten ein freier Zugang zur medizinischen Versorgung und eine Leistungsinanspruchnahme nach Bedarfskriterien ermöglicht werden kann. Schlüssig wäre es aus diesem Grund, das Leistungsfähigkeitsprinzip in der Finanzierung konsequent umzusetzen und historische, $z$. T. regressiv wirkende Regelungen, wie $z$. B. die Bindung der Beitragsbemessungsgrundlage an Einkommen aus unselbständiger Beschäftigung, entsprechend abzuwandeln. Folglich 
wäre die Finanzierungsgrundlage der solidarischen Krankenversicherung zu stärken, indem die Beitragsbemessungsgrundlage erweitert und die Beitragsbemessungsgrenze angehoben wird. Soweit dies zur Stärkung des Leistungsfähigkeitsprinzips und nicht zur vermehrten Beitragsschöpfung durchgeführt wird, ist im Sinne der Zielsetzung der Beitragssatz und somit die relative Belastung der Beitragszahler entsprechend zu senken.

Bei einer konsequenten Gestaltung der Finanzierung nach dem Leistungsfähigkeitsprinzip müßte auch die Versicherungspflichtgrenze aufgehoben werden. Deren Begründung leitet sich auf den eng mit dem Reziprozitätsgedanken verbundenen Solidarbegriff der Gründerjahre ab: Während bei den wirtschaftlich Stärkeren sowohl die erforderliche Einsicht als auch die Fähigkeit zur Eigenvorsorge vorausgesetzt wurde, setzte sich im Zuge der Einführung der GKV - im Gegensatz zu Bismarcks Zielen - die Ansicht durch, daß Geringverdiener mittels einer Pflichtversicherung zu Eigenvorsorge und gegenseitiger Hilfe gezwungen werden mußten. Diese im Hinblick auf EinkommensUmverteilungsbestrebungen eher regressiv wirkende Ausgliederung höherer Einkommen widerspricht dem heutigen Verständnis des Solidarprinzips und einer am Grundsatz des Leistungsfähigkeitsprinzips orientierten Finanzierungssystematik.

\subsubsection{Wettbewerb als Steuerungsinstrument}

Im Wettbewerb sind die Krankenkassen zugleich Anbieter und Nachfrager. Als Nachfrager, d. h. als Vertragspartner der Leistungsanbieter, obliegt es innen, das Produkt "Sicherheit im Krankheitsfall" zu gestalten. Als Anbieter konkurrieren sie nicht nur auf der Basis des Preises, sondern auch im Hinblick auf die Qualität und die Definition inres Produktes. Krankenkassen, die ihre Mittlerrolle zwischen den Leistungsanbietern und Versicherten kultivieren, können sich ihren Versicherten durch eine originäre und unverwechselbare Dienstleistung empfehlen. Der Preiswettbewerb zwingt die Kassen schon jetzt, effizientere Organisationsformen zu einer wirksamen Steuerung der Ausgabenentstehung zu entwickeln. Je weiter dabei die Möglichkeiten genutzt werden, durch sektorübergreifende Fallsteuerung mittels innovativer Vertragskonstruktionen, z. B. nach den $\S \S 63 \mathrm{ff}$., 73 und 73 a SGB V oder mittels eines direkt vom Kostenträger ausgeübten Fallmanagements, Effizienzverluste krankheits- und indikationsspezifisch abzubauen, desto stärker wird sich die konkrete Be- 
deutung des einheitlichen Leistungsrahmens in der GKV für die Versicherten relativieren.

Eine solche Differenzierung steht zwar in einem Spannungsverhältnis zum heutigen Solidarbegriff, sie muß aber nicht im $\mathrm{Ge}-$ gensatz dazu stehen, solange die Versicherten uneingeschränkt die Möglichkeit haben, einer Krankenkasse ihrer Wahl beizutreten. Der Wettbewerb der Krankenkassen bietet den Versicherten die Möglichkeit, über den Vergleich von Preis-LeistungsRelationen mit zu verfolgen, in welchem konkreten Umfang Leistungsanrechte künftig durch effizientere Organisationsformen im einzelnen gesichert werden können. Im Verhältnis zur Alternative einer schleichenden Rationierung ist ein gewisses Maß an „Produktdifferenzierung" in der GKV damit sogar wünschenswert. Dies setzt voraus, daß der Orientierungswandel der Kassen vom Status mittelbarer Staatsverwaltung hin zu einem kundenorientierten Dienstleister toleriert und unterstützt wird.

\subsubsection{Eigenverantwortung als Gestaltungselement}

Die Stärkung der Mittlerrolle der Krankenkassen im Wettbewerb dient der Sicherung einer möglichst großen faktischen Reichweite der Solidarität. Wird der Wettbewerb als Steuerungsinstrument wirksam, so ist es jedoch unumgänglich, daß es auch auf seiten der Versicherten zu einer Differenzierung unterschiedlicher Interessenlagen kommt und die Versichertengemeinschaften folglich zu „Präferenzgemeinschaften“ werden. Entscheidend ist es daher, daß die Versicherten ihre Wahl verantwortungsvoll treffen: Um nicht einen kontraproduktiven Wettbewerb zu fördern, ist folglich zwingend erforderlich, die Versicherten auch an den ökonomischen Folgen ihrer Wahlentscheidungen zu beteiligen.

Das Verhältnis von Solidarität und Eigenverantwortung muß somit neu bestimmt werden. Dabei darf der Begriff Eigenverantwortung jedoch nicht, wie in der aktuellen politischen Diskussion, in verkürzter Form ausschließlich für die Zuordnung bestimmter Finanzierungsanteile verstanden werden. Die Anhebung von Zuzahlungen verschafft zwar kurzfristige finanzielle Entspannung, sie dient jedoch nur marginal der Sicherung der faktischen Reichweite der Solidarität: Durch die schlichte Anhebung von Zuzahlungen werden einfach nur Risiken vor allem an diejenigen weitergegeben, denen eine solidarische Absicherung eigentlich zugute kommen sollte. Eigenverantwortung erscheint dabei als Kontrapunkt zur 
Solidarität. Folglich wird die entscheidende Frage, mit welcher Prioritätensetzung nämlich die solidarisch finanzierten Ressourcen einzusetzen sind, nicht unter expliziter Einbeziehung der Versicherten angegangen bzw. nicht dem Wirkungsbereich der Eigenverantwortung zugerechnet.

Um die Verantwortung der Versicherten auch in wesentliche Gestaltungsfragen der Solidarität einzubinden, sollte sich die finanzielle Eigenbeteiligung der Versicherten nicht auf Zahlungen im Zusammenhang mit konkreten Behandlungssituationen beziehen. Zudem sind die Versicherten als Patienten eher unfreiwillige Finanziers denn souveräne Nachfrager. Die Eigenbeteiligung sollte sich daher vielmehr auf die Wahl der Krankenkasse bzw. auf den Beitritt zu einer "Präferenzgemeinschaft" beziehen. Auf diese Weise würde sichergestellt, daß auch die Bemühungen der Kostenträger um Effizienz und Qualität des Leistungsangebots honoriert werden. Es würde ein Anreiz zur Förderung von Steuerungsmechanismen gesetzt, die eine verantwortungsvolle Inanspruchnahme der Versicherten beinhalten. Die Versicherten selbst wären an einer kritischen Beurteilung der im Rahmen der Steuerungsansätze zwischen Krankenkassen und Leistungserbringern gefundenen Definitionen und Auslegungsmuster des unbestimmten Rechtsbegriffes medizinischer Notwendigkeit interessiert.

\subsection{Das Modell des Krankenversicherungsfonds}

Die beschriebenen Entwicklungslinien lassen sich zu einem schlüssigen Modell zur Neudefinition der Solidarität verbinden, das hier nur in seinen Grundzügen vorgestellt werden kann ${ }^{77}$. Dabei ist sicherzustellen, daß die auf der Finanzierungsseite erhobenen Forderungen einer Stärkung wettbewerblicher Steuerungsmechanismen, die hier im Zentrum der Überlegungen zur Sicherung der faktischen Reichweite der Solidarität stehen, einer Stärkung der individuellen Verantwortung aller Versicherten nicht entgegenstehen.

Der Staat hat verfassungsgemäß die Pflicht, für einen Einkommensausgleich zu sorgen, durch den $u$. a. der Zugang zu einem

77 Zu Einzelheiten des Modells des Krankenversicherungs-Fonds und seiner Ableitung vgl. Stillfried D. Gesundheitssysteme im Wandel. Das Dilemma zwischen Bedarfskonzept und Eigenverantwortung: medizinische Grundsicherung als Reformperspektive? Bayreuth 1996 
ausreichenden medizinischen Versorgungsniveau gewährleistet werden kann. Es muß jedoch dringlichst ein ordnungspolitischer Rahmen für diese Transfermaßnahmen gefunden werden, der gewährleistet, daß die damit verbundene Abgabenbelastung reduziert oder zumindest ihr weiterer Anstieg wirksam begrenzt werden kann. Dabei besteht die Verantwortung der Politik nicht nur darin, die Belastungsgrenze der Versicherten zu definieren. Ihr muß auch die Entscheidung über das dann verfügbare Verteilungsvolumen klar zugerechnet werden. Diesbezüglich besteht im Rahmen des Sachleistungsprinzips der GKV keine Transparenz. Damit entsteht zugleich ein Verantwortungsvakuum für das Erreichen eines bestimmten faktischen Absicherungsniveaus. Zudem gefährden regulierende Eingriffe der Politik mit dem Ziel, bestimmte Versorgungsergebnisse sozialpolitisch sichern zu wollen, die Effizienz wettbewerblicher Steuerungsmechanismen.

Im Interesse einer klaren Verantwortungszuordnung zwischen Politik, Kostenträgern und Leistungserbringern sowie Versicherten wird deshalb im folgenden vorgeschlagen, die bisher im Beitragssatz der GKV verschmolzenen Funktionen „Risikoabsicherung" und "Umverteilung" durch einen Solidarausgleich auf zwei Stufen zu ersetzen und den zu einem bestimmten Abgabenniveau faktisch erreichbaren Umfang der Solidarität durch eine konsequente wettbewerbliche Neuorientierung auszuloten.

\subsubsection{Erste Stufe des Solidarausgleichs: Einkommensausgleich}

Auf der ersten Stufe wird zunächst ein Einkommensausgleich vorgenommen. Dafür zahlt jeder Bürger nach Maßgabe seiner wirtschaftlichen Leistungsfähigkeit in einen zweckgebundenen Fonds ein. Im Gegensatz zur Finanzierung der heutigen GKV, deren Beitragsbemessung die niedrigeren Einkommen aus abhängiger Beschäftigung überproportional belastet - obwohl deren Leistungen über $90 \%$ der Bevölkerung zugute kommen -, ist im Modell des Krankenversicherungsfonds das Leistungsfähigkeitsprinzip konsequent umzusetzen, d. h., die Beitragspflicht ist auf alle Enwerbstätigen und alle Einkunftsarten auszuweiten. Die Bemessungsgrundlage der Fondsabgabe wäre demnach die Summe der Einkünfte im Sinne des Einkommenssteuerrechts.

Aus dem Finanzvolumen des Krankenversicherungsfonds erhält jeder Bürger Anspruch auf eine Gutschrift in identischer Höhe, die 
ausschließlich zum Erwerb von Krankenversicherungsschutz verwendet werden kann. Bezogen auf die Lebenszeit sollte jedem ein in etwa gleiches Budget zur Verfügung stehen. Über diesen Einkommensausgleich wird folglich eine rein monetäre Grundsicherung definiert.

Dies eröffnet die Möglichkeit, die Festsetzung des Beitragssatzes zunächst völlig unabhängig von Fragen der Mittelverwendung im Wege einer zentralen politischen Entscheidung vorzunehmen, wobei sich die Politik ausschließlich am Kriterium einer akzeptablen Abgabenbelastung orientieren kann (Abbildung 5). Damit sie diese Entscheidung beherzt treffen kann, zieht sich die Politik, parallel zur Einführung des Krankenversicherungsfonds, im Zuge einer wettbewerblichen Neuordnung des Marktes für Krankenversicherungsschutz aus der unmittelbaren Verantwortung für die Steuerung im Gesundheitswesen zurück.

Abb. 5

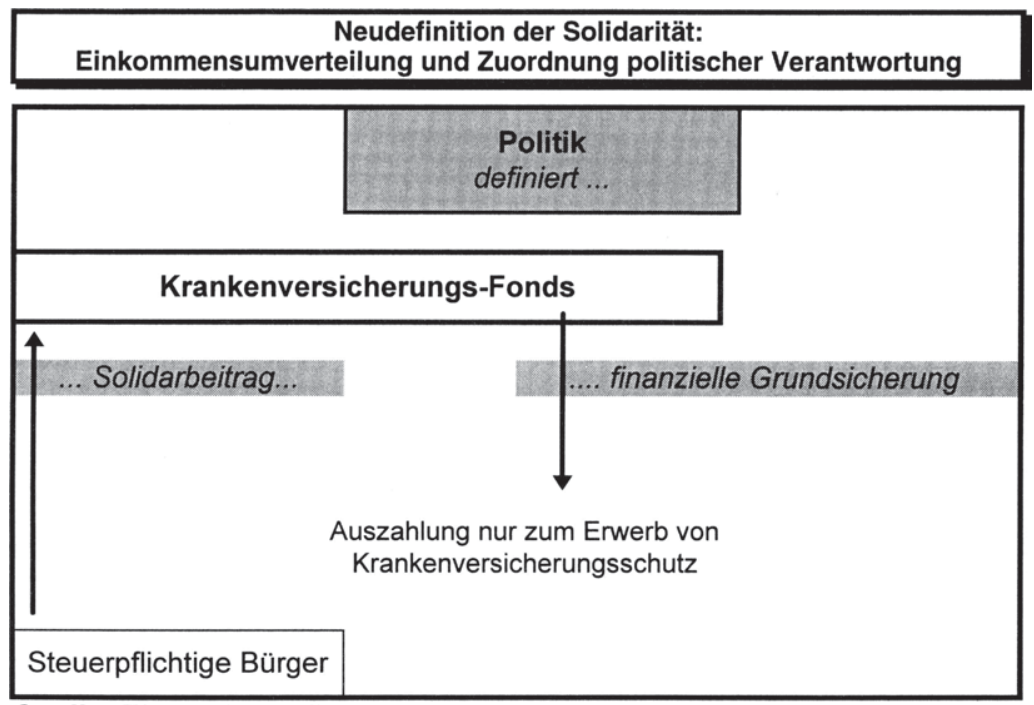

Quelle: Eigene Darstellung

\subsubsection{Zweite Stufe des Solidarausgleichs: Versicherungstechnischer Risikoausgleich}

Die zweite Stufe des Solidarausgleichs vollzieht sich auf der Ebene der Mitgliedschaft in einer konkreten Krankenkasse: Anstelle eines einkommensabhängigen Beitrags entrichten alle Versicher- 
ten einer Krankenkasse einen einheitlichen Mitgliedsbeitrag (Kopfpauschale) bei einem einheitlich je Krankenkasse definierten Leistungskatalog. Durch die Beibehaltung des Umlageverfahrens kommt es in jeder Versichertengemeinschaft zu einem versicherungstechnischen Risikoausgleich: Der Mitgliedsbeitrag richtet sich jeweils nach dem durchschnittlichen Risiko der Versichertengemeinschaft und nach Art und Umfang des Leistungsrahmens.

Die zweite Stufe des Solidarausgleichs wird durch zwei Regelungen gestärkt: Zum einen wird analog zur erweiterten Beitragspflicht die Versicherungspflicht auf alle Bürger erweitert. Zum anderen kann die unter Wettbewerbsbedingungen zu erwartende Ausdifferenzierung von Risikogruppen unter den beschriebenen Bedingungen leicht durch eine Regel begrenzt werden, derzufolge die Krankenkassen die Kostenübernahme für die Behandlung einer Krankheit nicht grundsätzlich ablehnen, ihre Leistungspflicht aber durch Bezug auf bestimmte Behandlungsleitlinien oder Versorgungsabläufe begrenzen können, sofern der Versicherte bei Vertragsschluß in verständlicher Weise darüber aufgeklärt wurde. Darüber hinaus wird von der bisher üblichen, engen leistungsrechtlichen Regulierung abgesehen.

\subsubsection{Konsistente Wettbewerbsordnung im Gesundheitswe- sen schaffen}

Die bestehenden Steuerungsdefizite, deren Ausdruck Überkapazitäten sowie Mängel in der Koordination und der Qualität der Versorgungsabläufe sind, lassen sich letztlich nur abbauen, wenn die Mittelallokation künftig nicht mehr von der Angebotsseite, sondern stärker nachfrageseitig gesteuert wird.

Dazu ist der von der Politik bereits eingeleitete Strukturwandel der Krankenkassen von reinen Verwaltungsapparaten zu wirtschaftlich voll verantwortlichen und innovativ handelnden Dienstleistungsunternehmen zu vervollständigen. Das Leistungs- und Vertragsrecht der Anbieter von Krankenversicherungsschutz (Krankenkassen und private Krankenversicherungen sind diesbezüglich gleichzustellen) muß dafür so reformiert werden, daß diese als wirklich gestaltende Mittler zwischen Versicherten und Leistungsanbietern fungieren können. Die Kassen werden jedoch nur unter einem entsprechenden Wettbewerbsdruck dazu veranlaßt werden, ihre Versorgungsangebote aktiv mitzugestalten und dadurch auch sehr viel genauer zu definieren (Abbildung 6). 
Abb. 6

\section{Neudefinition der Solidarität: \\ Wettbewerb und Spezifikation des Leistungskatalogs durch Nachfrageent- scheidungen}

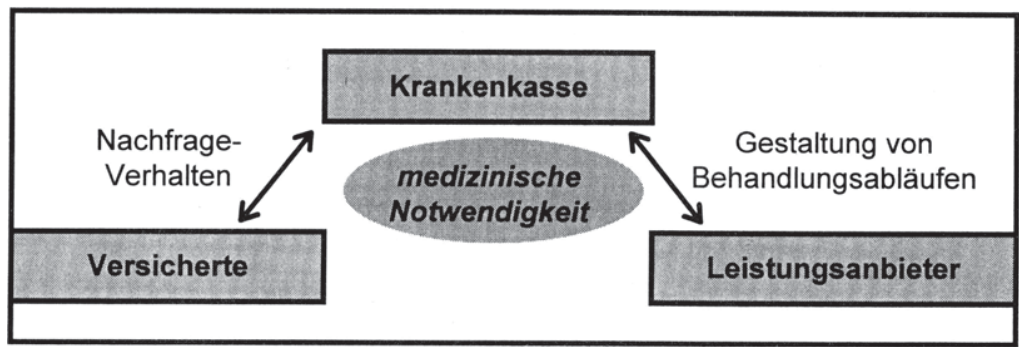

Quelle: Eigene Darstellung

Tendenziell wird so einer schleichenden Rationierung unter Ressourcenknappheit entgegengewirkt. Damit dieser Prozeß im Sinne des Grundsicherungsprinzips gefördert wird, ist die Vertragsfreiheit der Anbieter von Krankenversicherungsschutz durch folgende Auflagen einzuschränken:

- Die Versicherten haben uneingeschränkte Kassenwahlfreiheit; die Krankenkassen stehen unter Kontrahierungszwang und Diskriminierungsverbot.

- Die Leistungsdefinition erfolgt grundsätzlich über Satzungs/eistungen (einheitliche Leistungsdefinition für alle Versicherten einer Kasse), wobei die Krankenkassen ihre Leistungsangebote $u$. a. durch Angabe von Zuzahlungsregelungen, durch Angabe der Art und Bedingungen von Leistungsausschlüssen bzw. Negativlisten und durch Angabe von Eingrenzungen der Leistungspflicht innerhalb des angegebenen Leistungsrahmens, z. B. durch Positivlisten oder Behandlungsleitlinien, eindeutig qualifizieren müssen.

- Einmal jährlich können die Versicherten ihre Krankenkasse wechseln, wobei die Krankenkassen zuvor ihre Konditionen für das kommende Jahr verbindlich bekanntgeben müssen.

- Zum Schutz der Versicherten müssen die Krankenkassen eine Rückversicherung abschließen, wobei die Rückversicherer keinem Kontrahierungszwang unterworfen werden. 
Um eine nachhaltige Steuerungswirkung zu erzielen, reicht es nicht aus, dem einzelnen einen nur formalen Entscheidungsspielraum zwischen verschiedenen Krankenkassen zu eröffnen, die sich in den entscheidenden Wettbewerbsparametern, d. h. im Leistungs- und Vertragsrecht, nicht unterscheiden dürfen. Sinn dieser Neuordnung ist es daher, das Prinzip der Eigenverantwortung zu stärken: Der entscheidende Steuerungsimpuls wird letztlich von der Nachfrageentscheidung der Versicherten ausgehen.

\subsubsection{Fazit: Ein funktionsfähiger Rahmen für Eigenverantwortung der Versicherten}

Durch die Verflechtung der einzelnen Regelungsbestandteile entsteht ein funktionsfähiger Rahmen für die Ausübung von Eigenverantwortung der Versicherten (vgl. Abbildung 7).

Abb. 7

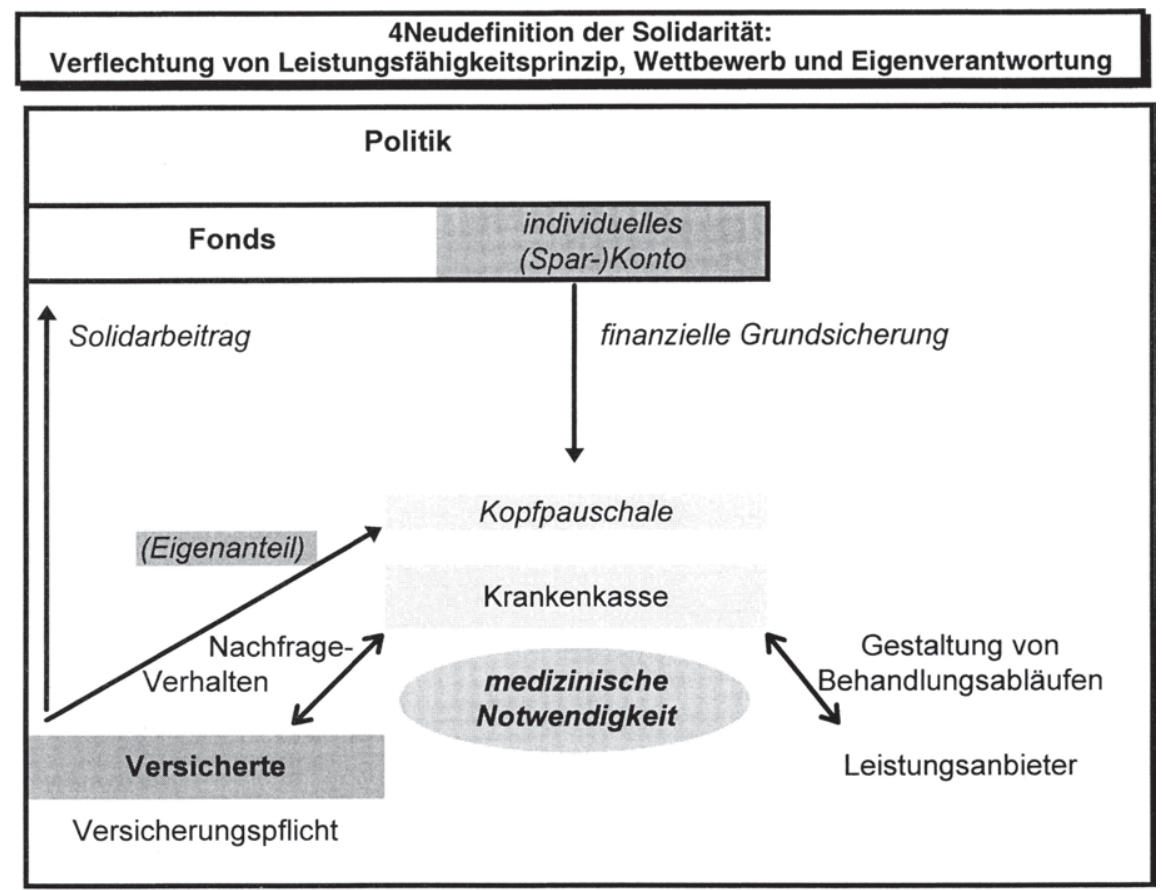

Quelle: Eigene Darstellung

Über ihre Nachfrageentscheidung wird den Versicherten ein Mitspracherecht bei der Definition ihres Leistungspaketes und der 
Inanspruchnahmemodalitäten eingeräumt. Durch ihre Gutschrift aus dem Krankenversicherungsfonds werden die Versicherten bei ihrer Nachfrageentscheidung finanziell unterstützt. Dennoch werden sie in ihrem Rückgriff auf das kollektive Unterstützungssystem, aber nicht aus ihrer individuellen ökonomischen Mitverantwortung entlassen, denn eine etwaige Differenz zwischen dem Mitgliedsbeitrag der gewählten Krankenkasse und der Gutschrift aus dem Krankenversicherungsfonds muß aus eigenen Mitteln beglichen werden. Umgekehrt werden nicht abgerufene Gutschriftsbeträge vom Fonds treuhänderisch verwaltet, so daß jeder Bürger auch über die zeitliche Verwendung seiner für die individuelle Risikovorsorge im Krankheitsfall reservierten Dispositionssumme entscheiden kann.

Im Gegensatz zu einer Abgrenzung über Leistungskataloge entspricht die monetäre Definition der Grundleistung auch der Tatsache, daß medizinische Notwendigkeit nicht objektiv definiert werden kann, sondern stets aus der normativen Bewertung individueller Bedürfnisse resultiert. Diese enthält zwingend subjektive Beurteilungsmomente, zumal die Inanspruchnahme medizinischer Dienstleistungen nicht ausschließlich der Vermeidung vorzeitigen Todes dient und sich der Nutzen vieler Leistungen des medizinischen Versorgungssystems erst im Kontext einzelner Lebenssituationen, also im schleichenden Übergang zu allgemeinen Bedürfnissen des Lebens eröffnet. Vor die Aufgabe gestellt, einen allgemeingültigen Grundleistungskatalog zu definieren, könnte der Gesetzgeber nur sehr grobe prioritäre Leistungsdefinitionen vorlegen. Angesichts der komplexen Entscheidungsmechanismen auf der parlamentarischen oder auch der Ebene korporativer Selbstverwaltungsorgane ist eine entsprechend spezifische, enge Auslegung dieser Definition nicht zu erwarten. Mit großzügigen Auslegungen, wie z. B. „... hat Anspruch auf ärztliche Versorgung", kann jedoch weder die Qualität noch die Wirtschaftlichkeit der Versorgung verbessert werden. Die Steuerungsmöglichkeiten im Rahmen einheitlicher Regelungen sind sehr begrenzt im Vergleich zu denen, die bei Vertragsfreiheit auf der Verhandlungsebene Kasse $\Leftrightarrow$ Leistungsanbieter denkbar sind. Dagegen dürften von einem Preis- und Qualitätswettbewerb der Krankenkassen aller Voraussicht nach auch neue Anreize zur Entwicklung kosten- und ausgabensenkender Innovationen in der medizinischen Versorgung ausgehen. Die treibende Kraft geht hierbei jedoch vom Verhalten der Versicherten aus. 
Diese dürften auf der Grundlage des beschriebenen Ordnungsrahmens in ihrem Nachfrageverhalten ein hohes Interesse an möglichst effizienten Versorgungsangeboten zum Ausdruck bringen. Wenn die Urteilskraft der Versicherten diesbezüglich immer wieder angezweifelt wird, so ist dem entgegenzuhalten, daß die Ausgangslage kaum geeignet ist, informierte Versicherte hervorzubringen, eine Lernkurve aber auch hier nicht ausgeschlossen werden sollte. Ein ausreichender Anteil der Versicherten dürfte trotz der Komplexität der Materie künftig zunehmend besser informiert sein, da Anbieter geeigneter Informationen, wie z. B. Stiftung Warentest, Verbaucherorganisationen sowie Rundfunkund Zeitschriftenmagazine, auf den Markt drängen werden, wenn die Wahl der Krankenkasse einen höheren Informationsstand erforderlich macht.

Die Neudefinition der Solidarität im Sinne des vorliegenden Reformvorschlags stärkt das dem Solidarbegriff ursprünglich zugrunde liegende Prinzip der Gegenseitigkeit. Im Kern geht es im vorgeschlagenen Reformmodell darum, die in der Entwicklung der GKV gewachsene Bedeutung der Einkommensumverteilung auf die Funktion subsidiärer Unterstützung zu konzentrieren, um den Begriff der Solidargemeinschaft wieder auf einen engeren gemeinsamen Handlungs- und Interessenzusammenhang beziehen zu können: die Gemeinschaft von Versicherten mit gleichen Versorgungspräferenzen.

Der Staat würde von einer Aufgabe entlastet, die inm zwar historisch zugewachsen ist, die er aber angesichts knapper Sozialhaushalte immer schlechter ausfüllen kann: die materielle Garantie eines versprochenen Versorgungsniveaus im Einzelfall auch sicherstellen zu können. Durch die rein monetäre Definition der Grundsicherung im Modell des Krankenversicherungsfonds wird die Garantie des versprochenen Leistungsniveaus Gegenstand des Vertragsverhältnisses zwischen Versicherungsnehmern und den Anbietern von Krankenversicherungsschutz. Die faktische Reichweite der gemeinsamen Ressourcen zu definieren, obliegt hingegen den Vertragspartnern in der Versorgung, d. h. Kostenträgern und Leistungsanbietern. Die staatliche Regulierung des Leistungsrahmens könnte auf wenige, sehr allgemeine Grundsätze begrenzt werden.

Für die Politik hätte der Vorschlag zudem den Vorteil, daß die Abgabenquote kontrolliert und mithin dem Lohnkostenargument 
im internationalen Wettbewerb Rechnung getragen werden kann, ohne notwendigerweise die Wachstumsdynamik des Gesundheitswesens - einem der arbeitsintensivsten Sektoren der Volkswirtschaft - durch rigide Kostendämpfungspolitik zu brechen, wie dies bei unveränderten Rahmenbedingungen in der GKV mittelfristig zu erwarten ist. Eine Fortsetzung der bisherigen "Industriepolitik“ mit Beitragsmitteln der GKV (z. B. Budgetanhebung zugunsten der Vergütung von Allgemeinärzten, Verzicht auf Positivliste, Krankenhaussonderopfer u. ä.) ist angesichts der Ressourcenknappheit auf Dauer nicht möglich. Die Vielfalt der Bedürfnisse nach medizinischer Versorgung läßt hingegen durchaus erwarten, daß viele Versicherte bereit sein werden, über die Gutschrift des Fonds hinaus Einkommensanteile für spezielle Versorgungsangebote einzusetzen.

Die größte Herausforderung des beschriebenen Modells dürfte in der mentalen Ablösung vom Zwei-Klassen-Denken bestehen. Während der einheitliche Leistungsrahmen im Kontext einer guten Finanzlage der GKV ungehinderten Zugang zum Füllhorn medizinischer Möglichkeiten versprach, steht die Einheitlichkeit des Leistungsrahmens im Kontext knapper finanzieller Ressourcen für die "Holzklasse" der Versorgung, da sich das Augenmerk auf die Personen richtet, die mit hoher Zahlungsfähigkeit und bereitschaft die Grenzen solidarisch finanzierter Versorgung überschreiten. Dabei beruht dieses Zwei-Klassen-Denken auf der intuitiven Unterstellung, eine angemessene medizinische Versorgung könne nur in einer Weise definiert werden. Im Ordnungsrahmen des Modells werden hingegen viele Wahl- und Gestaltungsmöglichkeiten von Krankenkassen, Leistungsanbietern und Versicherten realisiert werden. In Grenzen muß die Ausdifferenzierung der Leistungskataloge nach Präferenz- und damit auch nach Risikogruppen in einem wettbewerblich geordneten und durch die Nachfrageseite zu steuernden Versorgungssystem jedoch toleriert werden, um den Nachfrager tatsächlich mit in die ökonomische Verantwortung nehmen zu können.

Das Problem der Risikoselektion ist nicht ursächlich dem Wettbewerb anzulasten. Zugangs- und/oder Leistungsausschlüsse sind vielmehr eine Funktion relativer Ressourcenknappheit. Wird das Problem der Risikoselektion beim Zugang zu Versicherungsschutz durch die Formulierung von Zugangsrechten beseitigt, erscheint es meist in verdeckter Form, auch bei einheitlich verwalteten Leistungsstrukturen und einheitlicher Leistungsdefinition, 
beim Zugang zur Behandlung, z. B. in Form von Behandlungsausschlüssen bei der Inanspruchnahme der begrenzten Zahl britischer Dialyseeinrichtungen.

Dennoch wird sich die Glaubwürdigkeitskrise der herkömmlichen Gesundheitspolitik noch erheblich verschärfen müssen, bevor auf politischer Ebene Lernprozesse denkbar sind, die zu der hier vorgestellten, scheinbar revolutionären, aber gleichwohl nur die bisherigen Entwicklungstendenzen der GKV konsequent fortsetzenden Umgestaltung der sozialen Krankenversicherungssysteme führen können. 


\section{Einnahmenentwicklung der GKV und Finanzierungsoptionen:}

\section{Zusammenfassung der Arbeitsgruppe 1}

Dieter Cassel

Der Arbeitsgruppe I war die Aufgabe gestellt, die seit längerem beobachtbare Diskrepanz zwischen der Einnahmen- und Ausgabenentwicklung in der GKV zu thematisieren und darüber nachzudenken, durch welche gesundheitspolitischen Weichenstellungen die finanzielle Basis der GKV nachhaltig gestärkt werden kann. Hochgespannte Erwartungen, daß die Arbeitsgruppe als Diskussionsergebnis ein schlüssiges Konzept zur Lösung der Finanzierungsprobleme in der GKV vorlegen könnte, waren von vornherein unberechtigt: $\mathrm{Zu}$ komplex sind die Sachzusammenhänge, zu divergent die gesundheitspolitischen Zielsetzungen und zu gegensätzlich die Interessen der Beteiligten. Dies zeigte sich schon rasch nach den Eröffnungsstatements der Teilnehmer der Arbeitsgruppe, die mit vier Vertretern aus unterschiedlichen politischen Lagern (Herr Lohmann, Herr Reischl, Herr Weller und Dr. Zipperer), zwei Verbandsvertretern der GKV (Herr Dr. Klusen, Frau Walzik), einem Vertreter des PKV-Verbandes (Herr Dr. Uleer) und zwei Vertretern aus dem Bereich der Gesundheitsökonomie (Herr Prof. Dr. Münnich, Frau Prof. Dr. Pfaff) und Herr Laschet als Vertreter der Ärzte Zeitung hinreichend heterogen zusammengesetzt war, um einen von allen getragenen Lösungsvorschlag auszuschließen. Immerhin einigte sich die Gruppe sehr schnell darauf, ihre Aufgabenstellung unter folgenden Teilaspekten zu diskutieren:

- Wie werden sich Einnahmen, Ausgaben und Beitragssatz in der GKV längerfristig entwickeln, wenn die erkennbaren demographischen, medizintechnischen und wirtschaftsstrukturellen Tendenzen weiter anhalten und keine grundlegende Reform des Finanzierungssystems der GKV erfolgt?

- Ist es angesichts dieser Entwicklungsperspektiven erforderlich, das gesundheitspolitische Ziel der Beitragssatzstabilität aufzugeben, und welche Handlungserfordernisse ergäben sich dann für die Beitragssatzkalkulation einerseits und die Arbeitgeberbeiträge andererseits? 
- Sollte die Finanzierung der GKV nicht prinzipiell dadurch vom Arbeitseinkommen abgekoppelt werden, daß andere Einkunftsarten zur Beitragsbemessung herangezogen werden?

- Läßt sich die Finanzsituation der GKV dauerhaft dadurch verbessern, daß durch eine Erweiterung der Versicherungspflicht und Erhöhung der Beitragsbemessungsgrenze neue Versicherten- bzw. Beitragspotentiale erschlossen werden?

- Kann der bestehende Familienlastenausgleich ganz oder teilweise aus der GKV herausgenommen werden, so daß sich die Zahl der beitragspflichtigen Versicherten in der GKV erhöht?

- Und schließlich: Lassen sich die Finanzierungsprobleme der GKV durch mehr Risikoäquivalenz in der Beitragsgestaltung wie in der Leistungsinanspruchnahme entschärfen?

Entlastungen auf der Ausgabenseite - wie das Ausschöpfen von Rationalisierungspotentialen im Leistungsbereich oder eine Verringerung des Leistungskatalogs -, die ebenfalls zur Lösung der Finanzierungsmisere in der GKV beitragen könnten, wurden dagegen ausgeblendet, weil sie zum Aufgabenbereich der Arbeitsgruppen II und III gehören.

\section{Bestandsaufnahme: Anhaltende Finanzierungsprobleme}

Einig war sich die Arbeitsgruppe in der Einschätzung, daß die Finanzierungsproblematik der GKV über die aktuelle Situation hinaus auch künftig anhalten wird und sich sogar noch verschärfen könnte. Dies liegt zum einen daran, daß die Ausgabendynamik trotz aller Maßnahmen zur Kostendämpfung und zur Ausschöpfung von Wirtschaftlichkeitsreserven kaum zu bremsen ist: Der medizintechnische Fortschritt, der sich international durchsetze, wirke in Diagnose und Therapie weniger kostensenkend als vielmehr leistungsausweitend; die ungünstige demographische Entwicklung, verbunden mit einer steigenden mittleren Lebenserwartung und verbesserten Behandlungsmöglichkeiten, führe zu weiteren Ausgabenschüben; die nur unterdurchschnittliche Steigerung der Arbeitsproduktivität im dienstleistungsintensiven Gesundheitssektor führe zu einer kosteninduzierten Ausgabenbeschleunigung; und schließlich stiegen auch die Erwartungen der Versicherten in die Leistungsfähigkeit der Medizin, was eine ungebremste Nachfrage, insbesondere nach innovativen Diagnoseund Behandlungsmethoden, nach sich ziehe. 
Dem steht andererseits eine nur noch unterproportionale Erhöhung der Bruttolöhne und -gehälter als fast ausschließliche Beitragsbemessungsgrundlage entgegen (Abbildung 1). In diesem Zusammenhang wurde auf das vorausgegangene Impulsreferat von Prof. Münnich hingewiesen, in dem eine ganze Reihe von Faktoren genannt worden seien, die in der Vergangenheit national wie international nur noch zu einem relativ geringen Anstieg der Arbeitseinkommen beigetragen haben und inn im Zeitalter der Internationalisierung und Globalisierung künftig noch sehr viel stärker dämpfen werden. Stichwortartig wurden noch einmal die schrumpfende Bevölkerung, die sinkende Erwerbsdauer, die zunehmende Arbeitszeitverkürzung, die Veränderung der Beschäftigungsstruktur, die anhaltend hohe Arbeitslosigkeit sowie die relative Zunahme der Nichterwerbseinkünfte in der Wohlstandsgesellschaft genannt.

Abb. 1: Entwicklung der GKV-Ausgaben und Arbeitseinkommen, 1950-1996

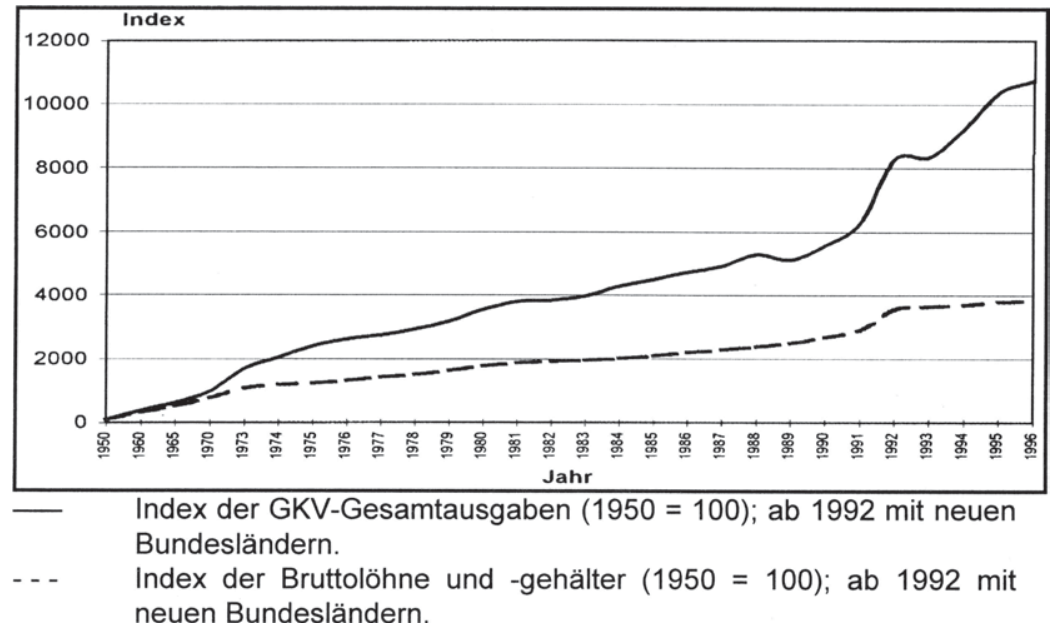

Quelle: GKV-Gesamtausgaben: 1950-1993 Bundesministerium für Arbeit und Sozialordnung, 1996; 1994-1996 BKK-Bundesverband, 1997; Bruttolöhne und -gehälter: Statistisches Bundesamt Wiesbaden, 1996, Fachserie 18, Reihe 1.2; eigene Berechnung.

Wenn nun aber die GKV-Ausgaben und die Arbeitseinkommen aufgrund der ihnen immanenten Triebkräfte derart weit auseinanderdriften, wie es die Abbildung 1 veranschaulicht, muß daraus zwangsläufig ein tendenziell steigender Beitragssatz folgen. 
Selbst wenn die Gesundheitspolitik darauf verweisen kann, daß ohne die Kostendämpfungsmaßnahmen der Vergangenheit der Beitragssatz schon heute bei 17 oder $18 \%$ liegen würde, ist doch unverkennbar, daß die trendmäßige Entwicklung in den letzten 25 Jahren deutlich nach oben zeigt (Abbildung 2).

Abb. 2: Beitragssatzentwicklung in der GKV (Westdeutschland), 1970-1996

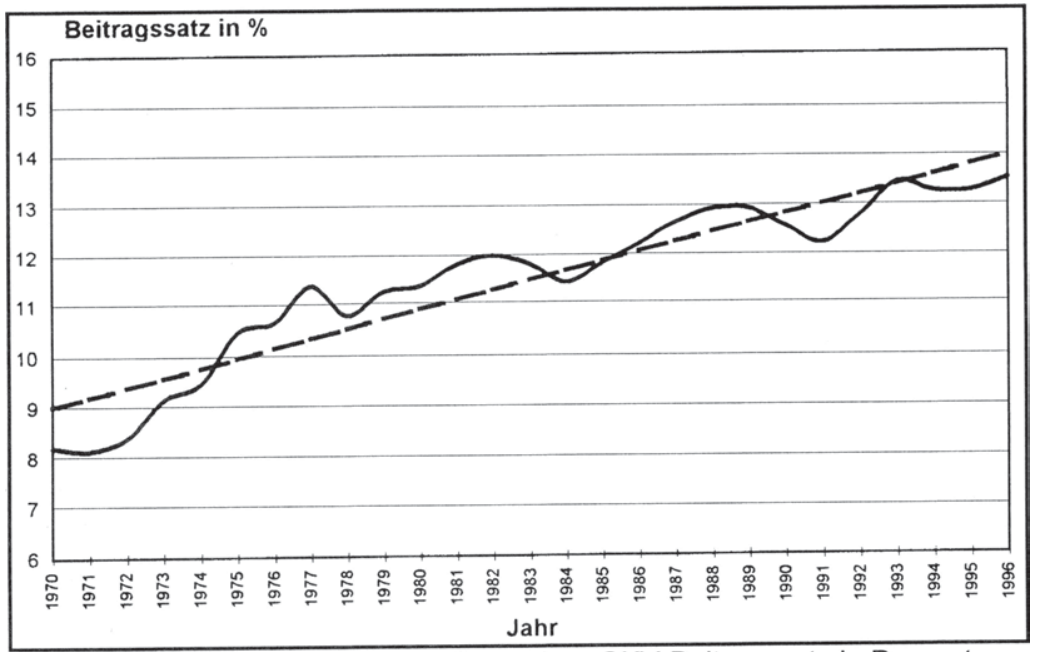

- Jahresdurchschnittlicher allgemeiner GKV-Beitragssatz in Prozent - - - Trendmäßige Entwicklung (lineare Regression)

Quelle: BKK-Bundesverband, 1997; eigene Berechnung

So aufschlußreich diese Ex-post-Betrachtung auch ist, viel wichtiger für die Einschätzung der künftig anstehenden Finanzierungsprobleme sei jedoch die Frage, ob die bisher zu beobachtenden Tendenzen weiter anhalten oder sich sogar noch verschärfen. Hierzu wurde auf eine Reihe von neueren Studien verwiesen (Knappe, 1995; Erbsland/Ried/Ulrich, 1997; Oberdieck, 1998), die unter Status-quo-Bedingungen die längerfristige Beitragssatzentwicklung bis zum Jahre 2040 abzuschätzen versuchen. Hebt man dabei ausschließlich auf die demographische Komponente ab, wie es Erbsland, Ried und Ulrich tun, ergibt sich ein nur relativ bescheidener Anstieg der Gesundheitsausgaben pro Kopf um etwa $20 \%$, was einen Anstieg des Beitragssatzes um 2,5 bis 3 Prozentpunkte zur Folge hätte. Wird dagegen in einem realistischeren Szenario die medizintechnische Entwicklung in ihrer Interdependenz mit der demographischen Entwicklung einbezogen, 
wie es in den Studien von Knappe und Oberdieck der Fall ist, sind Beitragssätze zu erwarten, die sich bis zum Jahr 2040 verdoppeln und über die 30-Prozent-Marke hinausgehen könnten (Abbildung 3).

Selbst wenn man derartigen Status-quo-Prognosen mit Vorbehalten begegnen muß, wie in der Diskussion angemerkt wurde, vermitteln sie jedoch die Einsicht, daß die GKV in ihrer derzeitigen Verfassung auf einer Tretmine steht, die bei jeder zusätzlichen Erschütterung hochgehen kann. Einmütig wurde daraus in der Gruppe der Schluß gezogen, daß es unbedingt notwendig sei, über grundlegende Reformoptionen nachzudenken, die über den Tag hinaus an den langfristigen Herausforderungen orientiert sind und unter Umständen auch die Grundprinzipien des gewachsenen Systems der GKV in Frage stellen müssen.

Abb. 3: Beitragssatzentwicklung in der GKV, 1970-2040

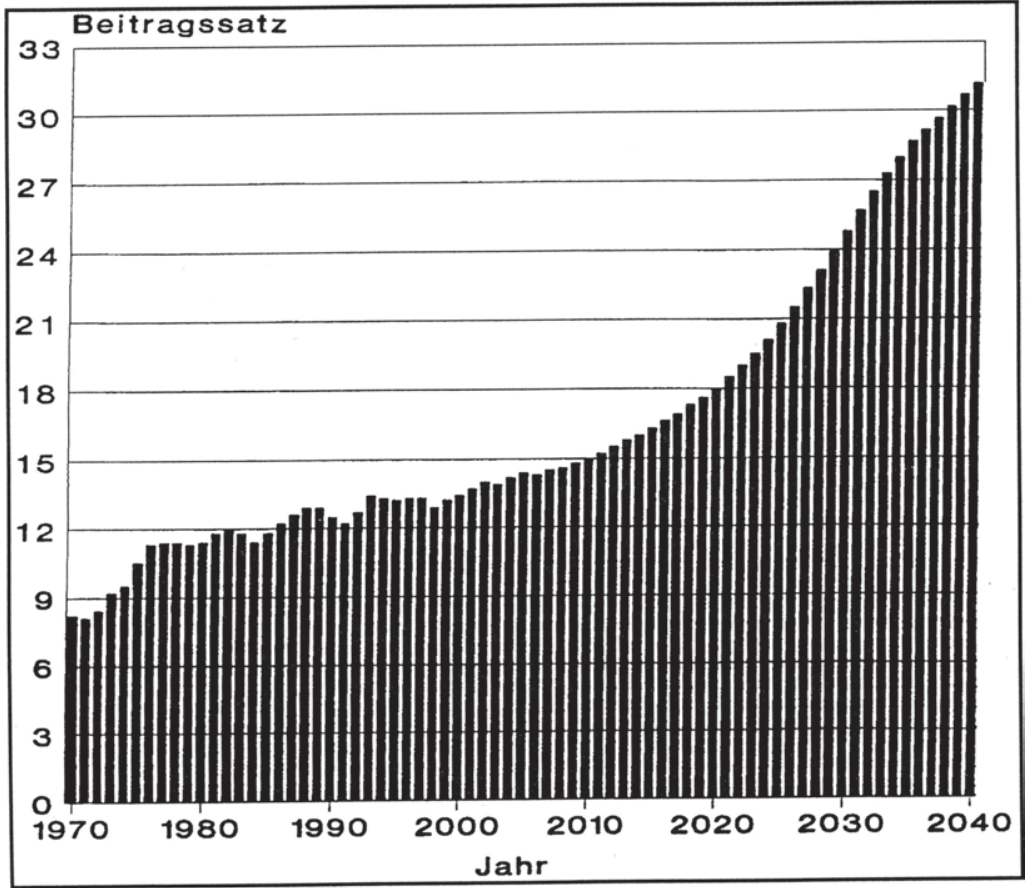

Jahresdurchschnittlicher allgemeiner Beitragssatz in Prozent; ab 1996: Status-quo-Prognose.

Quelle: Oberdieck, 1998 


\section{Reformoption I: Beitragssatzgestaltung}

Vor diesem Hintergrund wurde zunächst die Frage gestellt, ob es nicht gesundheitspolitisch opportun sei, das „Diktat der Beitragssatzstabilität" aufzugeben, so daß sich die Beitragssätze den offenbar schwer zu beeinflussenden Einnahmen- und Ausgabentrends entsprechend nach oben bewegen könnten. Die Gesundheitspolitik wäre von der Last befreit, mittels immer neuer dirigistischer Eingriffe stabile Beitragssätze einzufordern und im Fall notwendiger Beitragssatzerhöhungen ihre Glaubwürdigkeit zu verspielen. Ethisch gesehen wären die jetzt schon erkennbaren Rationierungen von Gesundheitsleistungen, die administrativ erzwungen werden, vermeidbar. Und ökonomisch könnten nach oben flexible Beitragssätze besser als Preissignal und Steuerungsinstrument eines wettbewerblichen GKV-Systems fungieren als die bisher auf administrativem Weg zurückgestauten Beitragssätze.

Hiergegen wurde aus gesundheitspolitischer Sicht argumentiert, daß es kein "Diktat der Beitragssatzstabilität" mit den inm zugeschriebenen Folgen gäbe; tatsächlich bedeute der in § 141 [2] SGB V festgeschriebene "Grundsatz der Beitragssatzstabilität", daß Beitragssätze durchaus erhöht werden könnten, aber eben nur dann, wenn die notwendige medizinische Versorgung auch unter Ausschöpfen von Wirtschaftlichkeitsreserven ohne Beitragssatzerhöhungen nicht mehr zu gewährleisten sei. Daraus wurde aus gesundheitsökonomischer Sicht die Schlußfolgerung gezogen, daß die Politik angesichts der künftig immer stärker auseinanderdriftenden Einnahmen- und Ausgabenentwicklung offenbar gezwungen sei, merkliche Beitragssatzerhöhungen hinzunehmen und die oben genannten Status-quo-Prognosen Realität werden zu lassen. Damit stelle sich aber um so mehr die Frage, wie sich angesichts der hälftigen Finanzierung der GKVBeiträge durch die Arbeitgeber die negativen Effekte aus der damit vorprogrammierten Erhöhung der Lohnzusatzkosten vermeiden lassen.

Als "sauberste" Lösung dieses Problems wurde von einigen Teilnehmern der Arbeitsgruppe vorgeschlagen, die Arbeitgeberbeiträge gänzlich abzuschaffen, indem sie einmalig dem Bruttolohn zugeschlagen werden. Dies würde zwar aktuell keinerlei Entlastungen bei den Lohnzusatzkosten bringen, würde aber künftig die Beitragssatzentwicklung gänzlich von den Lohnkosten abkop- 
peln, was im Interesse der Standortsicherung in Deutschland wünschenswert sei. Außerdem werde der Kassenwettbewerb intensiviert, sobald der gesamte Beitrag zur GKV von den Versicherten selbst zu tragen ist: Hierdurch gewännen Beitragssatzunterschiede zwischen den einzelnen Kassen ökonomisch an Gewicht, so daß die Bereitschaft zum Kassenwechsel im Interesse eines intensiven Preiswettbewerbs erhöht würde. Schließlich würde die Abschaffung der Arbeitgeberbeiträge auch Wettbewerbsverzerrungen innerhalb der Wirtschaft beseitigen, die dadurch entstehen, daß von den Kassen unterschiedlich hohe Beitragssätze eingefordert werden und sich Versicherte von billigen bzw. teuren Kassen in einzelnen Unternehmen, Branchen oder Regionen konzentrieren können. Auch wäre damit der kassenwahlbeschränkende Druck der Arbeitgeber auf ihre Arbeitnehmer, sich in bestimmten Kassen zu versichern, gegenstandslos.

Insbesondere von den Vertretern der Gesundheitspolitik und der GKV-Verbände wurde hiergegen heftig opponiert: Die Arbeitgeberbeiträge seien ein wichtiger Bestandteil des gewachsenen GKV-Systems und seien die Grundlage für den positiven Einfluß der Arbeitgeber auf die Geschäftspolitik der Kassen. Außerdem dürften die Arbeitgeber unter keinen Umständen aus der Verantwortung für eine gesundheitsfördernde Gestaltung der Arbeitsplätze und die Vermeidung von Unfällen und unnötigen Krankheitsrisiken entlassen werden. Dies strahle letztlich auch positiv auf die PKV aus, aus deren Sicht die Existenz der Arbeitgeberbeiträge ein Garant für die Funktionsweise des sozialen Sicherungssystems in Deutschland sei. Hinzu komme, daß die Kostenrelevanz der Arbeitgeberbeiträge in der GKV angesichts der sonstigen gesetzlichen und tarifvertraglich vereinbarten Lohnzusatzkosten maßlos übertrieben werde. Angesichts der Tatsache, daß die Erhöhung des Beitragssatzes um 1 Prozentpunkt die Lohnkosten nur um etwa 0,27 Prozentpunkte steigen läßt, sei die internationale Wettbewerbsfähigkeit der deutschen Wirtschaft durch Beitragssatzerhöhungen in der GKV kaum gefährdet und werde die paritätische Finanzierung von Wirtschaft und Politik zum "Popanz" gemacht. Allenfalls stelle der Arbeitgeberbeitrag ein Problem für die mittelständische Wirtschaft dar. Als problematisch wird aus dieser Sicht auch eingeschätzt, daß die paritätische $\mathrm{Fi}$ nanzierung immer mehr durch Zuzahlungen und Eigenleistungen der Versicherten im Krankheitsfall ausgehöhlt wird. Schließlich ergäbe sich ein weiteres Argument gegen den Arbeitgeberbeitrag, 
wenn weitere Einkunftsarten zur Finanzierung der GKV herangezogen werden sollten.

Gleichsam als Kompromiß wurde in der Arbeitsgruppe schließlich der Vorschlag diskutiert, den Arbeitgeberbeitrag auf dem jetzigen Niveau festzuschreiben, so daß künftige Beitragssatzerhöhungen in vollem Umfange von den Versicherten selbst zu tragen sind. Dabei wäre wünschenswert, den Arbeitgeberbeitrag zuvor auf den bundesdurchschnittlichen Beitragssatz zu normieren, um Wettbewerbsverzerrungen bei Unternehmen, die aus unterschiedlichen Beitragssätzen resultieren, zu vermeiden. Gefragt wurde auch, ob die Finanzierungsverpflichtung der Arbeitgeber nicht strikt auf das "medizinisch Notwendige" im Leistungskatalog auf dem heutigen Niveau zu beschränken sei. Anpassungen der Arbeitgeberbeiträge an künftige Entwicklungen blieben dann einer Überprüfung in mehrjährigem Abstand vorbehalten. Es wurde abschließend darauf hingewiesen, daß diese Kompromißlösung letztlich auch mit weitergehenden Optionen - wie "gespaltenen" Beitragssätzen, die nach der Höhe des Einkommens differenziert sind, Erweiterungen der Beitragsbemessungsgrundlage durch Einbeziehung anderer Einkunftsarten bis hin zu "nominellen“ Beiträgen, wie sie für die PKV typisch sind - vereinbar ist, was jedoch in der Diskussion nicht weiter vertieft wurde.

\section{Reformoption II: Heranziehung zusätzlicher Einkunftsarten}

Diese Reformoption erscheint auf den ersten Blick sehr attraktiv zu sein, weil sie die Beitragsbemessungsgrundlage erheblich zu verbreitern verspricht. Einleitend wurde hierzu bemerkt, daß die ausschließliche Heranziehung der Arbeitseinkünfte zur Finanzierung der Gesundheitsausgaben einschließlich der Lohnfortzahlung im Krankheitsfall bei Einführung der GKV durch die Bismarcksche Sozialgesetzgebung für die bis dahin weitgehend ungeschützten Industriearbeiter ein durchaus richtiges Konzept war. Nachdem jedoch $90 \%$ der Bevölkerung in der GKV versichert sind und ein wesentlicher Teil davon keine Erwerbseinkünfte bezieht, während andererseits der Anteil der Vermögenseinkünfte in der Wohlstandsgesellschaft ständig zunimmt, erscheint es schon aus Gründen der horizontalen Gerechtigkeit nicht mehr opportun zu sein, lediglich die Arbeitseinkommen und die sich daraus ableitenden Rentenbezüge zur Finanzierung der Gesundheitsausgaben in der GKV heranzuziehen. Ein entsprechender System- 
wechsel in der Finanzierung müsse freilich auch die Altersversorgung durch die gesetzliche Rentenversicherung mit einbeziehen.

Jedenfalls bestand in der Arbeitsgruppe weitgehend Einvernehmen darüber, daß bei entsprechender Anpassung der Versicherungspflicht- und Beitragsbemessungsgrenze erwägenswert sei, andere Einkunftsarten zur Finanzierung der GKV wie der gesetzlichen Rentenversicherung (GRV) heranzuziehen, um einerseits die Versicherten leistungsgerecht mit Beiträgen zu belasten und dadurch andererseits neue Finanzierungsquellen mit dem Ziel der Absenkung des Beitragssatzes zu erschließen. Hinsichtlich des letzteren Aspektes hatte ja schon Frau Prof. Pfaff in ihrem vorangegangenen Impulsreferat gezeigt, daß der Beitragssatz um knapp 3,4 Prozentpunkte abgesenkt werden könnte, wenn für alle Pflichtversicherten in den alten Bundesländern das Volkseinkommen als Bemessungsgrundlage herangezogen würde. Selbst wenn man diese Berechnung als äußerst hypothetisch einstuft, zeigt sie doch, welches Potential sich rein quantitativ hinter diesem Vorschlag verbirgt.

In der Diskussion wurden jedoch sogleich Bedenken geäußert, ob und inwieweit es sich hierbei um einen praktikablen Vorschlag handele. Zunächst sei zu fragen, welche Einkunftsarten hinsichtlich der voraussichtlichen Ausweichreaktionen der Versicherten überhaupt mit Aussicht auf Erfolg herangezogen werden können. $\mathrm{Zu}$ denken sei an das Problem der Kapitalflucht ebenso wie an die schattenwirtschaftliche Verheimlichung von Vermögen und der daraus fließenden Einkünfte. Schließlich habe es sich schon in der gegenwärtigen fiskalföderalistischen Debatte gezeigt, daß es im Zeitalter der Globalisierung und insbesondere im Zeichen des europäischen Binnenmarktes für mobile Faktoren immer leichter werde, sich der nationalen Steuer- und Abgabenbelastung zu entziehen. Darüber hinaus wurden insbesondere von den Vertretern der GKV-Verbände technische Schwierigkeiten bei der Beitragserhebung aus Nichterwerbseinkünften geltend gemacht: Wie sind die Angaben der Versicherten zu überprüfen? Sollen die Beiträge als Abschlagszahlung erhoben und erst später endgültig festgesetzt werden? Wie ist dann im Fall eines Kassenwechsels zu verfahren? Wie sollen ausstehende Beiträge eingetrieben werden? Sind nicht Wettbewerbsverzerrungen zwischen den Kassen zu erwarten, wenn sich einige Kassen weniger nachlässig als andere um die Heranziehung von Vermögensein- 
künften bemühen? Und wie läßt sich dann noch ein wettbewerbssichernder Risikostrukturausgleich praktizieren?

Obwohl diese Fragen nicht ausdiskutiert werden konnten, ergaben sich daraus in der Arbeitsgruppe erhebliche Vorbehalte gegen eine Einbeziehung zusätzlicher Einkunftsarten in die Beitragsbemessungsgrundlage der GKV. Diese Vorbehalte wurden noch durch das Argument verstärkt, daß diese Reformoption die GKV in die Nähe einer "Steuerfinanzierung" rücken würde, was einerseits dem Staat die Möglichkeit zu weiterer Einflußnahme geben und andererseits eine Diskussion um progressiv gestaltete Beitragssätze zur Wahrung der Prinzipien der "Steuergerechtigkeit" und "Leistungsfähigkeit" entfachen würde. Auch stünden dann noch andere Finanzierungsmodelle - wie zum Beispiel eine generelle Wertschöpfungsabgabe - zur Diskussion. Trotz aller Vorbehalte wurde es insgesamt jedoch als wünschenswert angesehen, zusätzliche Einkunftsarten zur Finanzierung der GKV heranzuziehen, um die künftige Beitragssatzdynamik zu dämpfen und dadurch die Problematik der Arbeitgeberbeiträge zu entschärfen, das Prinzip der Leistungsfähigkeit bei der Beitragsbemessung wieder mehr zur Geltung zu bringen und letztlich mehr horizontale Gerechtigkeit zu schaffen. $\mathrm{Ob}$ und inwieweit die genannten Vorbehalte dem wirklich entgegenstünden, müsse einer genaueren Analyse vorbehalten bleiben.

\section{Reformoption III: Zusätzliche Beitragspotentiale erschließen}

Falls zusätzliche Einkunftsarten zur Beitragsbemessung herangezogen würden, ergäbe sich eine potentielle Mehrbelastung nur für solche Versicherten der GKV, die bisher mit ihrem Einkommen unter der Beitragsbemessungsgrenze gelegen haben, während von der Beitragssatzsenkung, die man sich von der Verbreiterung der Beitragsbemessungsgrundlage erhofft, alle GKV-Mitglieder also auch die Besserverdienenden oberhalb der Beitragsbemessungsgrenze - profitieren. In diesem Falle müsse man schon aus Gerechtigkeitsgründen über eine entsprechende Anhebung der Beitragsbemessungsgrenze diskutieren. Aber auch ohne die Reformoption II sei erwägenswert, die Beitragsbemessungsgrenze der GKV bis auf die Grenze der GRV anzuheben, um zusätzliche Beitragspotentiale zu erschließen. Jedenfalls wurde diese Option von einigen Teilnehmern der Arbeitsgruppe zur Diskussion gestellt. Allerdings stieß dieser Vorschlag bei der Mehrheit auf heftige Bedenken: Ohne gleichzeitige Anhebung der Versicherungs- 
pflichtgrenze käme es zu einer Risikoentmischung in der GKV, weil gute Risiken zur PKV abwandern würden. Eine Erhöhung der Versicherungspflichtgrenze würde aber das Geschäftsfeld der PKV merklich einschränken, so daß der prinzipiell gewünschte Wettbewerb der beiden Systeme zur Disposition stünde. Fraglich sei auch, ob es vertretbar sei, den Besserverdienenden merklich höhere Beiträge ohne jede Gegenleistung abzuverlangen. Überdies stoße die Umverteilung in der GKV schon jetzt an ihre Grenzen, so daß es politisch nicht erwünscht sein könne, die "Leistungsträger" zu Gunsten der Bezieher niedrigerer Einkommen zusätzlich zu belasten. Schließlich sei auch offen, ob nicht Beitragserhöhungen bei den Besserverdienenden letztlich zu einer höheren Leistungsinanspruchnahme führen, so daß der Nettoeffekt einer Anhebung der Beitragsbemessungsgrenze fraglich sei.

Ebenso kontrovers diskutiert wurde der Vorschlag, die Beitragsbemessungsgrenze beizubehalten, die Pflichtversicherungsgrenze dagegen abzuschaffen, um letztlich alle abhängig Beschäftigten der GKV zuzuführen. Diese Regelung ginge freilich auch wieder zu Lasten der PKV, die dadurch in ihrer Existenz bedroht wäre. Angesichts der Tatsache, daß die PKV für gleiche ärztliche Leistungen etwa das dreifache zahlt wie die GKV, wäre überdies fraglich, ob die Erschließung neuer Versichertenpotentiale aus der PKV überhaupt zu einem positiven Nettoeffekt für die GKV führe. Mit den gleichen Argumenten wurde auch der Vorschlag abgelehnt, Selbständige und Beamte in die GKV zu überführen. Letztlich hat die Diskussion über die Anhebung der Beitragsbemessungs- und Versicherungspflichtgrenze sowie über die Einbeziehung neuer Personenkreise in die GKV gezeigt, daß alle diese Optionen die Grenze zwischen PKV und GKV merklich verschieben würden, ohne daß mit Sicherheit gesagt werden könne, ob dadurch ein hinreichend positiver Finanzierungseffekt gewährleistet ist.

Schließlich hat sich die Arbeitsgruppe unter diesem Punkt auch noch mit der Reform des Familienlastenausgleichs in der GKV beschäftigt. Als Reformoption wäre daran zu denken, den Familienlastenausgleich völlig aus der GKV herauszunehmen und aus Steuermitteln zu finanzieren. Dies würde letztlich bedeuten, daß für jedes Familienmitglied eine eigene Beitragspflicht zu begründen ist, was die GKV zweifellos auf eine breitere finanzielle Basis stellen würde. Hiergegen wurde jedoch massiv argumentiert, daß 
die beitragsfreie Versicherung von Familienangehörigen ein zentraler Bestandteil jeder sozialen Krankenversicherung sei und folglich nicht zur Disposition stehen dürfe. Allenfalls sei diskutabel, Ehegatten, die weder Kinder erziehen noch Familienmitglieder pflegen, beitragspflichtig zu machen. So wünschenswert eine solche Regelung unter Gerechtigkeitsgesichtspunkten auch sei, finanziell würde sie jedoch nicht allzuviel bringen.

\section{Reformoption IV: Mehr risikoäquivalente Beitragsgestaltung}

Als letzten Punkt hat die Arbeitsgruppe noch die Frage diskutiert, ob und inwieweit eine Lösung der Finanzierungsproblematik von einer stärker risikoäquivalenten Gestaltung der GKV zu erwarten ist. Diskutiert wurden hierbei insbesondere mögliche Effekte von Zuzahlungen, Selbstbehalten und Formen der Beitragsrückgewähr. Zunächst wurde aus gesundheitsökonomischer Sicht anerkennend hervorgehoben, daß diesbezüglich die beiden NOGs mehr Gestaltungsfreiheit gebracht hätten, was hinsichtlich des intendierten GKV-Wettbewerbs zu begrüßen sei. Andererseits wurde kritisch eingewandt, daß damit Elemente Eingang in die GKV gefunden hätten, die traditionell der PKV vorbehalten gewesen seien. Einige Teilnehmer der Arbeitsgruppe lehnten denn auch die Beitragsrückgewähr und die Selbstbehalte für die GKV rundweg $a b$, weil sie nicht risikoäquivalent zu kalkulieren seien, keine Steuerungsfunktion im GKV-System hätten und letztlich voll zu Lasten der Kranken gingen. Andere Teilnehmer wiesen dagegen darauf hin, daß mit diesen Instrumenten durchaus Kostensenkungseffekte erzielt werden könnten, wenn es gelänge, die Versicherten dadurch in ihrer Leistungsinanspruchnahme zu einem rationaleren Verhalten zu bewegen. Soweit dadurch eine Senkung der Leistungsausgaben zu erreichen sei, werde die Finanzierungsproblematik durch diese Optionen zweifellos entschärft.

In diesem Zusammenhang wurde provokant auch die Frage gestellt, ob sich die GKV unter dem absehbaren Finanzierungsdiktat nicht letztlich zu einem "Drittelsystem" entwickeln werde: Ein Drittel der Ausgaben finanziere der Versicherte über die Beiträge, ein weiteres Drittel zahlten die Arbeitgeber mit ihren Arbeitgeberbeiträgen und ein letztes Drittel käme schließlich von den Kranken über die Zuzahlungsregelung. Letztlich sei dies unter den eingangs dargestellten Bedingungen eine notwendige Entwick- 
lung, sofern es nicht gelänge, die Finanzierungsproblematik über andere Reformoptionen zu lösen.

Zusammenfassend läßt sich somit feststellen, daß die Diskussion der denkbaren Reformoptionen zur Lösung der langfristigen Finanzierungsprobleme der GKV zwar durchweg sachlich, aber eben doch in entscheidenden Punkten äußerst kontrovers geführt wurde. So war die Arbeitsgruppe weit davon entfernt, ein in sich geschlossenes und noch dazu erfolgversprechendes Lösungskonzept zu entwickeln, was von vornherein - wie anfangs erwähnt -auch nicht zu erwarten war. Offenbar gibt es in dieser Frage keinen „Königsweg", der den divergenten Zielsetzungen und Interessen entspricht und gleichzeitig zum Ziel führt. Weder gestellt noch diskutiert wurde die Frage, ob nicht eine durchgreifende Deregulierung des GKV-Systems genügen würde, um allein durch die Verringerung der gesundheitspolitisch agierenden Bürokratien und Interessenvertretungen einen merklichen Entlastungseffekt zu erzielen. Möglicherweise wäre die Einsicht konsensfähig gewesen, daß auch im Gesundheitswesen „viele Köche den (Reform-)Brei verderben". Mit dieser eher ironischen Bemerkung möchte ich den Bericht aus der Arbeitsgruppe I beenden und mich zum Schluß vielmals bedanken: bei den Teilnehmern für die äußerst anregende und facettenreiche Diskussion und bei Herrn Dr. Erbsland für die gedächtnisstützenden Protokollnotizen.

\section{Literatur}

Knappe, E., Auswirkungen des demographischen Wandels auf den Gesundheitssektor, in: Oberender, P. (Hrsg.), Transplantationsmedizin. Ökonomische, ethische, rechtliche und medizinische Aspekte, Baden-Baden (Nomos) 1995, S. 11-41.

Erbsland, M.; Ried, W.; Ulrich, V., Die Auswirkungen der Bevölkerungsstruktur auf Ausgaben und Beitragssatz der gesetzlichen Krankenversicherung. Vortrag auf dem ZEW-Symposion „Ansätze zur Reform des Steuer- und Sozialversicherungssystems" vom 10.-11. März 1997 in Mannheim.

Oberdieck, V., Beitragssatzexplosion in der Gesetzlichen Krankenversicherung (GKV)? Demographische und medizintechnische Determinanten der Beitragssatzdynamik und ihre reformpolitischen Implikationen, Duisburger Diss., Hamburg (S+W-Verlag) 1998. 


\section{Chancen und Risiken von Strukturverträgen und Modellvorhaben: \\ Zusammenfassung Arbeitsgruppe 2}

Gerhard Schulte

Wir hatten in der zweiten Arbeitsgruppe das Vergnügen, uns mit Reformoptionen zu beschäftigen, die schon im Fünften Buch des Sozialgesetzbuches stehen, und damit auf relativ gesicherten Grundlagen zu diskutieren. Wir haben acht Themen aus dem Komplex „Strukturverträge und Modellvorhaben" diskutiert, die ich vorweg kurz nennen darf:

1. Sind die Ziele des Gesetzgebers richtig plaziert?

2. Sind die Instrumente, die die beiden gesetzlichen Möglichkeiten bieten, die richtigen Instrumente zur Erreichung der Ziele?

3. Wie ist die Rolle der Vertragspartner bei Strukturverträgen und Modellvorhaben zu bewerten, insbesondere die Sonderstellung der Kassenärztlichen Vereinigung?

4. Wie ist die Rolle des Versicherten gestaltet?

5. Handelt es sich insbesondere bei Strukturverträgen um „Verträge zu Lasten Dritter"?

6. Wie ist die Erfolgsbeteiligung $\mathrm{zu}$ bewerten, die sowohl im Strukturvertrag als auch im Modellvorhaben für Ärzte bzw. Versicherte vorgesehen ist?

7. Handelt es sich bei den Strukturverträgen und den neuen Modellvorhaben um einen Teil des Wettbewerbs in der gesetzlichen Krankenversicherung, oder sind sie dem Prinzip der Einheitlichkeit der Versorgung zuzuordnen?

8. Haben wir noch Zeit für Modellvorhaben?

\section{Ziele des Gesetzgebers}

Die im $\S 63 \mathrm{ff}$. und im $\S 73$ a SGB V formulierten Ziele, Qualität und Wirtschaftlichkeit der Versorgung zu verbessern, werden allgemein begrüßt. Die Weiterentwicklung der Gesundheitsversorgung durch die Vertragspartner in der gesetzlichen Krankenversicherung war bisher nur in sehr engem Rahmen möglich. Daß der 
Gesetzgeber hier Tore geöffnet hat, wird als wesentlicher Aspekt der 3. Stufe der Gesundheitsreform gewertet.

\section{Konvergenz von Zielen und Instrumenten}

Ob die zur Verfügung gestellten Instrumente der Modellvorhaben und Strukturverträge die richtigen Ansätze zur Erreichung der Ziele darstellen, ist schon ein etwas schwierigerer Sachverhalt.

Die Arbeitsgruppe hat sich zunächst mit der Frage beschäftigt: „Wo liegt überhaupt der Unterschied der Modellvorhaben zu den Strukturverträgen?" Es ist die Auffassung geäußert worden, daß ein Strukturvertrag, so wie er im $\S 73$ a dargestellt ist, auch als Modellvorhaben nach $\S 63 \mathrm{ff}$. umgesetzt werden könnte. Rechtlich spricht allerdings viel dafür, daß der Gesetzgeber den $\S 73$ a als Lex specialis gestaltet hat, also der Gedanke vernetzter Praxisstrukturen in Verbindung mit kombinierten Budgets nur in der Form von Strukturverträgen vereinbart werden kann. Diese Frage hat durchaus eine praktische Bedeutung, da Krankenkassen, die sich mit der Frage Modellvorhaben oder Strukturvertrag beschäftigten, auch prüfen werden, welches der geeignete Weg ist. Der Strukturvertrag erfordert ein Handeln der Kassenart in einer Region, während das Modellvorhaben der einzelnen Krankenkasse die Möglichkeit gibt, tätig zu werden. Je nach Struktur der Verbände und Krankenkassen gibt es hier unterschiedliche Ansätze.

Die Möglichkeit, schnell zu einem Ergebnis zu kommen, ist bei Strukturverträgen und Modellvorhaben durchaus unterschiedlich. Denn die Tatsache, daß Modellvorhaben in der Form der Satzungsänderung von den Aufsichtsbehörden genehmigt werden müssen, könnte möglicherweise eine beträchtliche Zeitverzögerung zur Folge haben. Es wurde in diesem Zusammenhang etwa das Beispiel der Betriebskrankenkassen in Bayern genannt. Für ein Modellvorhaben komplexerer Natur, an dem ca. 200 Betriebskrankenkassen in der Bundesrepublik Deutschland beteiligt sein müßten, weil ansonsten nicht eine ausreichende Versichertenzahl zustande kommt, müßten sämtliche Aufsichtsbehörden der Bundesrepublik Deutschland an den Satzungsänderungen von 200 Betriebskrankenkassen beteiligt werden. Und wer schon das Vergnügen hatte, solche Dinge auszuhandeln, der kann sich sicher vorstellen, daß zwar möglicherweise keine 17, aber doch 5 unterschiedliche Meinungen zustande kommen. Dies ist je nach 
Struktur der Kassenart schon von der Zeitschiene her von Bedeutung.

Die Arbeitsgruppe hat sich in diesem Zusammenhang mit der Frage beschäftigt, ob Versorgungsmodelle, die vor dem 01.07.1997 von gesetzlichen Krankenversicherungen zum Teil mit und zum Teil ohne Vertragsgrundlagen mit den Kassenärztlichen Vereinigungen auf den Weg gebracht worden sind, ab dem 01.07. nach dem neuen Recht behandelt werden müßten. Hier war die Antwort, daß die vor dem 01.07. vereinbarten Verträge oder auf Satzungsrecht beruhenden Modelle auf der Grundlage des alten Rechts fortgeführt werden können. Wenn sich allerdings im Laufe der Modellvorhaben Veränderungen ergeben, müssen diese nach neuem Recht beurteilt werden. Zum Beispiel haben wir etwa die Regelung, daß Modellvorhaben über medizinische Sachverhalte, die der Bundesausschuß Ärzte und Krankenkassen schon negativ entschieden hat, nicht mehr möglich sind. Es wäre denkbar, daß ein begonnenes Modellvorhaben zu alternativen Therapiemethoden nach dem 01.07.1997 vom Bundesausschuß negativ entschieden wird. Dann müßte nach Auffassung der Arbeitsgruppe das Modellvorhaben abgebrochen werden.

Weiterhin wurde die Frage gestellt, ob eine Beschränkung, die der Gesetzgeber bewußt für Modellvorhaben eingebaut hat, nämlich der Ausschluß einer Wirksamkeitsprüfung von medizinischen Behandlungsmethoden oder Arzneimitteln, dennoch unter dem Aspekt struktureller Wirkungen möglich ist? Können etwa im Rahmen von Modellvorhaben unter dem Aspekt des Wirtschaftlichkeitsgedankens noch Sachverhalte erprobt werden, die sich zwar nicht mit der Wirksamkeit der Behandlungsmethode beschäftigen, wohl aber mit den wirtschaftlichen Folgen einer schon bewerteten medizinischen Leistung? Sie können sich sicher denken, daß in diesem Punkt die Sichtweise unterschiedlich war. Die Krankenkassen haben durchaus ein Interesse, in den Bereichen, wo es im Gesetzgebungsverfahren den Beteiligten gelungen ist, den größten Schutzzaun aufzubauen, nämlich im Arzneimittelbereich, gleichwohl unter dem Aspekt der Wirksamkeit Modellvorhaben durchführen zu können.

\section{Die Rolle der Vertragspartner der GKV}

Die Rolle der Vertragspartner und insbesondere die Sonderstellung der Kassenärztlichen Vereinigungen sind sehr ausführlich 
diskutiert worden. Zunächst kann man feststellen: wichtige Vertragspartner der gesetzlichen Krankenversicherung, die Krankenhausträger, haben nicht die gleiche Möglichkeit, wie sie in der ambulanten ärztlichen Versorgung besteht. Die Situation der Krankenkassen ist insoweit günstiger. Sie können, wenn sie medizinische Leistungen erproben wollen, in vielen Bereichen bei gleichen Sachverhalten überlegen, ob sie dies nicht vertraglich z. B. mit 20 Krankenhäusern vereinbaren. Hier brauchen sie nur 20 Krankenhäuser von 2.600 in der Bundesrepublik Deutschland, und Krankenhausgesellschaften können sich nicht in die Vertragsebene einklagen. Andererseits wurde festgestellt, daß die ambulante Versorgung mit einer Vielzahl von Ärzten natürlich nicht so ohne weiteres mit der stationären Versorgung zu vergleichen ist und deswegen die Intention des Gesetzgebers, eine Atomisierung der Modellvorhaben in der ambulanten ärztlichen Versorgung zu verhindern und eine Kanalisierung über die KVen zu erreichen, verständlich ist.

Weiterhin wurde die Frage diskutiert, welche Bedeutung im Bereich der Modellvorhaben die Regelung des Gesetzes hat, auch gegen den Willen eines KV-Vorstandes eine Mehrheitsmeinung betroffener Ärzte herbeiführen zu können, um einer Sperrwirkung der Kassenärztlichen Vereinigung zu begegnen. Hier ist die Auffassung der Vertreter der gesetzlichen Krankenversicherung gewesen, daß es sehr problematisch ist (etwa in dem Sektor Nuklearmedizin), mit wenigen Ärzten, die eine Mehrheit gegen ihre eigene KV zustande bringen könnten, ein Modellvorhaben auf den Weg zu bringen. Die Krankenkasse muß ja mit der KV auch andere Probleme lösen als die der Nuklearmedizin, etwa Fragen der ärztlichen Gesamtvergütung oder der Arzneimittelrichtgrößen. Sie muß sich deshalb sehr wohl überlegen, ob sie eine solche Konfliktsituation eingehen will. Schon deshalb wird es auf seiten der gesetzlichen Krankenversicherung eine beträchtliche Zurückhaltung geben.

Im Zusammenhang mit der Verhinderung der Atomisierung von Modellvorhaben ist allerdings auch gesagt worden, daß eine Reihe von Modellvorhaben nur dann funktionsfähig und aussagekräftig sein kann, wenn sie in kleineren Einheiten durchgeführt wird. Dies wiederum führt zur Folgefrage, ob denn ein Recht auf Teilnahme niedergelassener Ärzte an vereinbarten Modellvorhaben oder Strukturverträgen besteht und ob sich ggf. Ärzte einklagen können. Grundsätzlich war die Meinung die, daß derjenige 
Arzt, der die Vertragskriterien erfüllt, auch ein Recht auf Teilnahme hat. Dies korrespondiert nicht unbedingt mit der Notwendigkeit, in kleineren Einheiten zu arbeiten. Die Arbeitsgruppe hat es aber mehrheitlich für möglich und sinnvoll gehalten, bei einem größeren Interesse an einer sinnvollen Maßnahme ab einer bestimmten Größenordnung in zwei Einheiten zu operieren. Die Vertreter der gesetzlichen Krankenversicherung sind der Meinung, daß es aus der Sicht der Krankenversicherung durchaus sinnvoll ist, Modellvorhaben auf breitere Grundlagen zu stellen. Denn sie wollen nicht nur in einzelnen kleineren Bereichen modellhaft in den nächsten acht Jahren etwas erproben, sondern so bald wie möglich eine Flächenwirkung erreichen. Die Gestaltungsfreiheit der Vertragspartner reicht nach Klärung der Arbeitsgruppe aus, um die Funktionsfähigkeit von Modellvorhaben zu sichern.

Die Rolle anderer Vertragspartner und insbesondere auch die Situation der pharmazeutischen Hersteller wurden intensiv erörtert. Hier war die Meinung, daß Hersteller im System der gesetzlichen Krankenversicherung nur auf der zivilrechtlichen Ebene partizipieren können. Wenn die Vertragspartner es für sinnvoll halten, etwa in Fragen der Arzneimittelversorgung auch den Sachverstand der pharmazeutischen Industrie einzubeziehen, steht dem nichts entgegen. Und es ist in bestimmten Bereichen auch sinnvoll. Aber man verläßt insoweit dann die sozialrechtliche Ebene. Pharmazeutische Hersteller sind genauso einzuordnen wie andere Beratungsorganisationen, die die Vertragspartner zur Umsetzung von Modellen beteiligen könnten.

Zur Rolle der Apotheken im Zusammenhang mit Modellvorhaben und Strukturverträgen, die wiederum Vertragspartner innerhalb der GKV sind, muß man feststellen, daß sie durch inre möglicherweise geschickte Taktik erreicht haben, daß Kernbereiche der Arzneimittelversorgung von den Modellvorhaben im Rahmen des $\S 63 \mathrm{ff}$. ausgeschlossen sind. Naturgemäß ist deswegen der Rahmen der Beteiligung von Apotheken an Modellvorhaben relativ schmal. Das Interesse von Apothekerverbänden und einzelnen Apothekern, in Modellvorhaben einbezogen werden zu können, korrespondiert nicht mit der Rechtslage.

\section{Die Rolle des Versicherten}

Die Rolle des Versicherten bei Strukturverträgen und Modellvorhaben ist eine ausgesprochen starke. Im $\S 73$ a ist deutlich for- 
muliert, daß der Versicherte nur auf der Grundlage einer freiwilligen Entscheidung für ein solches Strukturmodell einbezogen werden kann. Darüber hinaus ist die Arbeitsgruppe zu dem Ergebnis gekommen, daß die Formulierungen des $\S 63 \mathrm{ff}$. im Zusammenhang mit der notwendigen Satzungsänderung faktisch auch dazu führen, daß sich der Versicherte im Einzelfall zur Teilnahme an einem Modellvorhaben erklären muß. Denn der Versicherte hat ein Kündigungsrecht dann, wenn der Leistungsbereich tangiert ist; was regelmäßig der Fall sein wird.

Es ist weiterhin darauf hingewiesen worden, daß sich Erwartungen des Versicherten in solchen Modellvorhaben nicht einfach darstellen lassen. Es gibt keinen Durchschnittsversicherten, aber möglicherweise doch zwei Gruppen von Versicherten, die gerade bei Modellvorhaben und Strukturverträgen eine unterschiedliche Interessenlage haben. Die einen sind die überwiegend Gesunden, die vielleicht ein- oder zweimal im Jahr akut behandelt werden oder eine Vorsorgeuntersuchung durchführen lassen. Die anderen sind die Langzeitkranken, die chronisch Kranken und die Älteren, die schon aufgrund ihrer gesundheitlichen Situation ein stärkeres Interesse an den Behandlungsabläufen haben. Und das, was möglicherweise dem einen recht und teuer ist, kommt dem anderen nicht unbedingt entgegen. Hierzu verfügt die gesetzliche Krankenversicherung im Augenblick über keinen konkreten Erfahrungshintergrund.

\section{Strukturverträge zu Lasten Dritter?}

$\mathrm{Ob}$ insbesondere Strukturverträge "Verträge zu Lasten Dritter" sind, ist eine Frage, die aus der Diskussion während des Gesetzgebungsverfahrens bekannt ist. Die Arbeitsgruppe hat grundsätzlich gesagt, die Frage ist berechtigt, nur falsch formuliert, weil jeder Vertrag Ziele hat. Und Ziele eines Vertrages betreffen immer andere, auch negativ, jedenfalls aus ihrer Sichtweise. Es ist sicher ehrlich zu sagen, daß sich Strukturverträge, so wie sie im $\S 73$ a formuliert sind, in erster Linie gegen den aktuellen Umfang der stationären Versorgung richten. Denn der Grundgedanke vernetzter Praxisstrukturen ist es, wesentlich mehr und sinnvollere ärztliche Behandlung in der ambulanten Ebene zu fördern, als es heute der Fall ist. Wenn Krankenkassen das wollen, dann müssen sie die Ärzte für diese zusätzliche Leistung bezahlen. Und genau dies ermöglicht der Strukturvertrag, nämlich eine Gesamtbetrachtung der Ausgaben, die durch niedergelassene Ärzte be- 
einflußt werden können. Strukturverträge werden folglich, wenn sie erfolgreich sind, Gewinner und Verlierer haben.

Aber auch Kassenärztliche Vereinigungen können Dritte in diesem Sinne sein. Denn insbesondere die Modellvorhaben ermöglichen es beispielsweise den Krankenkassen, mit Krankenhäusern Vereinbarungen zu treffen, die die Interessenlage der niedergelassenen Ärzte berühren. Hier entsteht dann die Rechtsfrage, ob in solchen Bereichen, in denen die ambulante Ebene im Rechtssinne betroffen ist, die Kassenärztlichen Vereinigungen beteiligt werden müssen. Das Abgrenzungskriterium ist die Definition der Krankenhausbehandlung, die im $\S 39$ lediglich formal definiert wird. Sie setzt nicht auf der ärztlich-medizinischen Seite an, sondern Krankenhausbehandlung ist eine Behandlung, die ambulant nicht möglich ist und die teilstationär nicht erbracht werden kann. Also wird für ein Modellvorhaben zwischen Krankenhausträgern und gesetzlicher Krankenversicherung der Versuch gemacht werden, den Begriff der teilstationären Behandlung möglichst weit zu fassen, um auch in bestimmte Bereiche ambulanter Versorgung hereingreifen zu können. Ob dadurch dann die ambulante Medizin betroffen ist und insofern ein Mitwirkungsrecht der KV besteht, konnte in der Diskussion nicht geklärt werden. Es wurde aber darauf hingewiesen, daß es natürlich möglich und sinnvoll ist, im Rahmen von Modellvorhaben dreiseitige Verträge darüber zu schließen, daß bestimmte, im Augenblick in unserer Rechtsordnung der ambulanten Ebene zugeordnete Leistungen im Krankenhaus erbracht werden und umgekehrt bestimmte Krankenhausleistungen sinnvollerweise in den ambulanten Bereich überführt werden.

\section{Erfolgsbeteiligungen}

Die Möglichkeit der Erfolgsbeteiligung in Strukturverträgen für Ärzte und in Modellvorhaben für Versicherte wurde kontrovers diskutiert. Zunächst muß festgestellt werden, daß die Erfolgsbeteiligung der Ärzte im $\S 73$ a der entscheidende Ansatz ist, um hier etwas zu erreichen. Das muß dem Gesetzgeber klar gewesen sein. Deswegen sollte man sich dazu auch bekennen. Es wurde in der Diskussion darauf hingewiesen, daß bei einer Umfrage bei 3.000 bayrischen Ärzten durch die KV Bayern die Frage gestellt worden ist, was die Ärzte von Strukturverträgen halten. Und die Antwort war: $28 \%$ der Ärzte halten Strukturverträge für sinnvoll, $20 \%$ nicht und $50 \%$ sind noch unentschlossen. Auf die 
Frage, was müßte denn für Ärzte bei der Umsetzung von Strukturverträgen herauskommen, haben $80 \%$ geantwortet: eine wirtschaftliche Verbesserung, bezogen auf die augenblickliche Situation. Das heißt nüchtern betrachtet, den Ärzten einen Strukturvertrag anbieten zu wollen, der wirtschaftlich nichts bringt, wäre ein vergebliches Bemühen.

Was die Versicherten angeht, besteht zunächst einmal das Problem, daß in einem Strukturvertrag eine Erfolgsbeteiligung für Versicherte nicht vorgesehen ist, es sei denn, eine bessere Qualität. Es besteht allerdings die Möglichkeit, auf der Grundlage eines Strukturvertrages ein Modellvorhaben nach $\S 63$ aufzubauen, das eine Erfolgsbeteiligung der Versicherten vorsieht. Wenn man überhaupt an eine solche Erfolgsbeteiligung der Versicherten denkt, dann muß man einen Weg finden, Kranke zu beteiligen und nicht Gesunde, die ohnehin nicht zum Arzt gehen. Dies könnte in der Weise geschehen, daß im Verhältnis zur individuellen Zuzahlung des Patienten im Vorjahr ein bestimmter Anteil zurückgezahlt wird. Damit würde nicht nach dem Motto 10 oder 20 DM pro Monat und Nase vorgegangen, sondern man würde denjenigen, die über eine Zuzahlung den höchsten Eigenanteil geleistet haben, einen Teilbetrag im Verhältnis zur Inanspruchnahme zukommen lassen. Das wären diejenigen Teilnehmer an einem Modellvorhaben, die durch vernünftiges Verhalten auch am meisten bewirken können. Die Mehrheit der Arbeitsgruppe war der Meinung, dieses widerspräche nicht dem gesetzlichen Ansatz, daß Zuzahlungen nicht vermindert werden dürfen. Denn es handelt sich hier nicht um eine Absenkung der Zuzahlung beispielsweise von $9 \mathrm{DM}$ auf $6 \mathrm{DM}$ bei Arzneimitteln, sondern es ist nur der Berechnungshintergrund für die Erfolgsbeteiligung nach $\S 63 \mathrm{ff}$.

\section{Wettbewerb oder Einheitslösung}

Die Arbeitsgruppe war der Meinung, daß es für den Arzt in der Tat schwer ist, im medizinischen Bereich zweierlei Maßstäbe anzulegen. Die Vertreter der GKV waren sich im klaren darüber, wenn sie qualitätsorientierte Modellvorhaben mit Kassenärztlichen Vereinigungen vereinbaren, daß dann alle anderen Krankenkassen Nutznießer sind. Wenn etwa für bestimmte Krankheitsbilder Behandlungsleitlinien zur Anwendung kommen sollen, dann wird der Arzt, der von der Sinnhaftigkeit der Behandlungsleitlinie überzeugt ist, sie nicht nur bei Versicherten der vertrags- 
schließenden Krankenkasse, sondern bei allen seinen Patienten anwenden. Insofern sind alle Krankenkassen in der gleichen Ausgangssituation. Sie nehmen in Kauf, daß sich gute Ansätze im System der gesetzlichen Krankenversicherung verbreiten.

Mehrheitlich war die Gruppe der Meinung: Strukturverträge und Modellvorhaben sind ein wichtiger Teil des gewollten Wettbewerbes in der gesetzlichen Krankenversicherung, und deswegen darf man trotz der gerade beschriebenen Schwierigkeit nicht verlangen, alles einheitlich und gemeinsam laufen zu lassen. Da die GKV wenige seriöse Wettbewerbsparameter hat, wäre es auch falsch, so etwas zu fordern. Krankenkassen würden sich auf den Schnickschnack der letzten Jahre besinnen, den sie mühsam mit Hilfe des Gesetzgebers zurückgedrängt haben. Man muß, was Modellvorhaben und Strukturverträge angeht, sich zum Wettbewerb bekennen.

\section{Zeit für Modellvorhaben}

Es bestand Einvernehmen: Das Angebot, das der Gesetzgeber gemacht hat, kann von den Vertragspartnern nicht abgelehnt werden. Niemand sollte sich auf den Standpunkt stellen, einmal abzuwarten, was machen denn andere; oder wird der Vertragspartner von sich aus aktiv; oder man betrachtet in den nächsten acht Jahren die Entwicklungen und Trends und wartet dann weitere zwei Jahre auf die Auswertung der wissenschaftlichen Begleitung. Und entscheidet schließlich im Jahre 2008, wie es denn mit den Modellvorhaben weitergeht.

Es bestand darüber hinaus innerhalb der Arbeitsgruppe auch Einvernehmen, daß Modellvorhaben und Strukturverträge nicht nur in der Provinz plaziert werden können. Die Vertragspartner müssen in die Ballungszentren, wo die größten Probleme bestehen, mit Modellvorhaben und Strukturverträgen gehen. Man kann zweifelsohne in Rendsburg starten, aber Berlin, Hamburg, München, Köln, Dortmund und Stuttgart nicht auslassen. Der Strukturvertrag nach $\S 73$ a ist der sinnvollste Weg, um tatsächlich auch in der Fläche etwas zu erreichen. Die Gesundheitspolitik wird die Partner der GKV spätestens Mitte der nächsten Legislaturperiode fragen, was sie aus den Möglichkeiten der dritten Reformstufe gemacht haben, und es ist außerordentlich wichtig für alle, darauf eine plausible Antwort zu haben. 


\section{Der Leistungskatalog im Lichte des Solidaritätsprinzips: \\ Zusammenfassung Arbeitsgruppe 3}

Hans-Jürgen Firnkorn*

\section{Der Solidaritäts begriff}

Wenn der Leistungskatalog der GKV im Licht des Solidaritätsprinzips überprüft werden soll, muß man sich zuvor darüber klar werden, was dieses Prinzip alles umfaßt. Es hat einen erheblichen quantitativen und qualitativen Bedeutungswandel in den letzten hundert Jahren erfahren: Aus rund $5 \%$ der Bevölkerung, die die Bismarcksche Sozialgesetzgebung zunächst zwangsversicherte, sind heute um die $70 \%$ zwangs- und ca. $20 \%$ freiwillig Versicherte geworden.

Qualitativ hat sich das System vom reinen Lohnersatz (Krankengeld), der sich sowohl beim Beitrag als auch bei der Leistung im Krankheitsfall nach der Lohnhöhe richtete, zu einem umfassenden System für die Deckung fast aller Kosten entwickelt, die im Krankheitsfall entstehen können. Diese Kosten werden solidarisch getragen, d. h., die Beiträge werden nicht nach Risikogruppen erhoben, sondern nach der Höhe der Lohneinkommen. Dadurch zahlen

- diejenigen, die ein höheres Lohneinkommen haben, für diejenigen, die einen geringeren Lohn haben,

- diejenigen, die ein geringeres Krankheitsrisiko haben, für diejenigen, deren Risiko höher ist (d. h. die Gesünderen für die Kränkeren),

- die Kinderlosen und Kinderarmen für die Kinderreichen,

- die Singles für die Familien,

- die Sorgsamen für die weniger Sorgsamen.

Die Leistungen sind jedoch (mit Ausnahme des Krankengeldes) für alle gleich: Unabhängig von Alter, Geschlecht, Beschäftigungsstatus, Gesundheitszustand, Beitragshöhe haben alle GKVVersicherten im Krankheitsfall den gleichen Anspruch auf Versor-

\footnotetext{
"Der Berichterstatter dankt Herrn Prof. Dr. Dr. h.c. Michael Arnold für zahlreiche Korrekturen und Anregungen.
} 
gung mit notwendigen und wirtschaftlichen Leistungen nach dem jeweiligen Stand der Medizin. Der Anspruch ist öffentlichrechtlicher Natur, er unterliegt damit dem Gleichheitsgebot des Grundgesetzes und kann nicht wie im Privatrecht abgedungen werden.

Um dies zu gewährleisten, ist eine Zwangsversicherung notwendig, die indes bis heute nicht die ganze Bevölkerung umfaßt, sondern nur jenen Teil, deren Einkommen unter der Beitragsbemessungsgrenze liegt. Personen, deren versicherungspflichtiges Einkommen über der Beitragsbemessungsgrenze liegt, können sich u. $U$. anderweitig günstiger versichern.

Die Beiträge zur GKV werden - seit den 50er Jahren hälftig vom Arbeitnehmer und Arbeitgeber gezahlt. Der Einbeziehung der Arbeitgeber in das System der sozialen Sicherung liegt die Überlegung zugrunde, daß dieser an einer raschen Gesundung seines Arbeitnehmers ein Interesse hat, was Einfluß auf die Gestaltung der Arbeitsbedingungen haben kann, und er im übrigen eine Fürsorgepflicht für seinen Arbeitnehmer wahrzunehmen hat.

Die solidarische Krankenversicherung verfolgt neben dem gleichen Krankheitsschutz für alle GKV-Versicherten also erhebliche Umverteilungsziele. Diese duale Zielsetzung erzwingt eine Organisationsform, bei der die Elemente Zwangsversicherung, einkommensabhängige Beiträge und öffentlich-rechtliche Regulierung der Gewährung von Versicherungsleistungen einander bedingen. Freiwillige Versichertengemeinschaften bilden sich ja nur dort, wo Gruppen mit Mitgliedern vorhanden sind, die ungefähr gleichen Risiken unterliegen und die diese Risiken durch entsprechend hohe Beiträge auch absichern können oder die sich auf Teilabsicherungen und Risikoausschluß einigen. Ein rein freiwilliges System der Krankenversicherung führte wohl mit Sicherheit dazu, daß ein Teil der Bevölkerung gar keinen und ein weiterer Teil nur einen eingeschränkten Schutz im Krankheitsfall hätte. Die Effektivität der Versorgung für den größten Teil der Bevölkerung sicherzustellen scheint nur möglich, wenn die Umverteilung der Lasten in das System einbezogen wird. Dies führt zum Versicherungszwang und der Regulierung von Beiträgen und Leistungen. Dies ist nur in einem öffentlich-rechtlichen System möglich, das seinerseits an das Gleichheitsgebot des Grundgesetzes gebunden ist. 
Läuft nun dieses System aus dem Ruder, weil seine Ausgaben aus welchen Gründen auch immer - von den Einnahmen nicht mehr gedeckt werden können, dann gibt es folgende Optionen zur Lösung der Probleme:

- der Kreis der Anspruchsberechtigten wird verändert,

- die Ausgaben werden den Einnahmen (Budgetierung) oder die Einnahmen den Ausgaben (Beitragssatzerhöhungen) angepaßt mit der Folge von Niveauveränderungen bei den Leistungen oder bei den Beiträgen oder bei beiden,

- einzelne Elemente des Solidarprinzips, wie der Gleichversorgungsanspruch oder die Umverteilung, werden ganz oder teilweise aufgegeben.

Die Arbeitsgruppe 3 befaßte sich entsprechend dem vorbeschriebenen Rahmen und in Arbeitsteilung mit den beiden anderen Diskussionsgruppen im wesentlichen mit den Fragen:

- ob es berechtigt ist, die heutige GKV für $70 \%$ der Bevölkerung zwangsweise vorzuschreiben und die Möglichkeit einer freiwilligen Mitgliedschaft beizubehalten?

- ob und wie die vorhandenen Probleme in der GKV durch Korrekturen des Leistungskataloges in der Art und dem Umfang oder durch Korrekturen des Solidarprinzips gelöst werden können?

\section{2. Änderung des Kreises der Anspruchsberechtigten}

Im Zuge des Ausbaus der GKV wurde nicht nur der Kreis der Versicherten und daher Anspruchsberechtigten erweitert, sondern auch Art und Umfang der Leistungsanrechte mit der Folge, daß nicht nur der wirtschaftlich Schwache für seine Gesundung notwendige Leistungen erhält, sondern auch der nicht auf Fremdhilfe Angewiesene Präferenzgüter. Folgerichtig wurde von einigen Diskutanten eine grundlegende Änderung des bisherigen Solidarverständnisses gefordert und vorgeschlagen, zwischen "need" und "demand" zu unterscheiden. Das Solidaritätsprinzip würde dann nur noch dort gelten, wo es um "need" geht, also um Not und Bedürftigkeit auf der einen und um das Notwendige auf der anderen Seite. Das jetzige GKV-System sei nicht nur auf die Be- 
friedigung des - wie unscharf auch immer zu bestimmenden Bedarfs an Gesundheitsleistungen für etwa $90 \%$ der Bevölkerung ausgerichtet, sondern finanziere auch die Befriedigung von darüber hinausgehenden Bedürfnissen noch solidarisch. Der Inanspruchnahme des immer größeren und differenzierteren Leistungsangebots stehen keine individuell wirksam limitierenden Faktoren entgegen, so daß das System immer wieder aus dem Gleichgewicht geraten muß, wobei es keine Mechanismen der Selbststabilisierung hat, vielmehr durch staatliche Interventionen „saniert" werden muß.

Da nun in keinem anderen Lebens- und Wirtschaftsbereich in unserer Gesellschaft für diejenigen, die nicht Not leiden, eine materiell gleichförmige und vom Standard her hohe Versorgung vorhanden ist oder auch nur ernsthaft gefordert wird (die Verhältnisse im steuerfinanzierten Bildungsbereich liegen wohl anders), stellt sich die Frage, mit welcher Begründung eine solidarische Krankenversicherung für $90 \%$ der Bevölkerung aufrechterhalten wird, von der mindestens die Hälfte, wenn nicht gar zwei Drittel der Versicherten aufgrund ihrer wirtschaftlichen Lage ausreichend Selbstverantwortung übernehmen und Selbstvorsorge betreiben könnten. Wenn man dieser Annahme zustimmt, kommt man rasch zu dem Vorschlag, die solidarische Krankenversicherung wieder auf die tatsächlich Hilfsbedürftigen und auf die Fälle von unstrittiger Bedürftigkeit (need) auszurichten und Überforderungen durch zu hohe GKV-Beiträge mit Steuergeldern aufzufangen. Das Solidaritätsprinzip würde dadurch nicht aufgegeben, denn es beinhaltete weiterhin eine Umverteilung von oben nach unten, und sei es über die Steuer. Es beendete aber die Umverteilung von unten nach oben, wie sie das jetzige System mit sich bringt, da Beiträge nur von Einkommen aus abhängiger Beschäftigung und nur innerhalb einer Beitragsbemessungsgrenze erhoben werden. Beschäftigte mit niedrigem Gesamteinkommen aus abhängiger Beschäftigung - und das sind im wesentlichen die Bedürftigen - werden relativ stärker belastet als Personen mit höherem Gesamteinkommen aus mehreren Einkommensarten. Dies kann schwerlich als sozial gerecht gesehen werden.

Der heftig diskutierte Vorschlag wurde letztlich aus folgenden Gründen abgelehnt:

Die Zurückführung der GKV auf eine Armenkasse erschüttert das gewachsene Krankenversicherungssystem als System einer um- 
fassenden kollektiven Sicherung im Krankheitsfall für fast die gesamte Bevölkerung so stark, daß der Verlust an sozialem Frieden alle Allokationsvorteile der Änderungen aufzehrt.

Es ist keineswegs sicher, daß der Gesamtumfang an finanziellen Aufwendungen für das Gesundheitswesen geringer wird, wenn ein großer Teil davon in die private Vorsorge abwandert, wie das Beispiel der USA zeigt. Nur die Rechtfertigung für den höheren Aufwand ist problemloser, wenn dieser sich als Ausgleich von Angebot und Nachfrage auf einem von Präferenzen und Anbieterwettbewerb bestimmten Markt ergibt.

Die Effektivität eines auf die wirtschaftlich schwächeren Bevölkerungsteile eingeschränkten Systems ist geringer als das derzeitige System, da ihm tendenziell gute Risiken fehlen, die jetzt zur Mitfinanzierung herangezogen werden. Außerdem ist es äußerst unwahrscheinlich, daß der Staat gegenwärtig aus Steuermitteln für die Defizitfinanzierung aufkommen würde, wo sogar rechtlich zugesagte Leistungen per Gesetz gestrichen oder gekürzt werden ( $z$. B. Krankenhausinvestitionsmittel).

Schließlich ist die jetzige GKV (wie das gesamte System der sozialen Sicherung in Deutschland) ein System gesellschaftlicher Partizipation, die verloren ginge, was destabilisierend wirken würde. Von dem System profitieren nicht nur die kranken Versicherten, sondern auch sämtliche Anbieter und Beschäftigten im Gesundheitssektor, der einen beachtlichen Teil zum Bruttosozialprodukt beisteuert.

Die genannten Argumente wurden im einzelnen aber nicht eingehend diskutiert, so daß weder ein Konsens noch ein klarer Dissens festgestellt werden kann. Zwar war allen Beteiligten klar, daß beim jetzigen System zwischen dem - positiv bewerteten ausreichenden Versicherungsschutz für den größten Teil der Bevölkerung und der diesen voraussetzenden Umverteilung und der - negativ gesehenen - Überinanspruchnahme (und dem Überangebot) unterschieden werden muß; aber es herrschte eine diffuse Furcht, daß die als politisch und sozial wünschenswert angesehene Sicherung der Vorsorge im Krankheitsfall so eng mit den solidarischen Umverteilungsregeln zusammenhängt, daß deren Aufgabe auch die Sicherstellung einer ausreichenden Versorgung für alle oder wenigstens die meisten Bürger gefährdet. Die Hypothese vom Zusammenhang zwischen einer als vorhanden ange- 
sehenen Versorgungseffektivität (im Sinne einer gleichmäßig hohen Versorgung für $90 \%$ der Bevölkerung) und dem solidarischen Umverteilungssystem wurde in der Diskussion aber weder ernsthaft bezweifelt noch nachhaltig bestätigt.

Man einigte sich darauf, daß ein solidarisches System nicht ohne Zwang auskommen kann und daß dies beinhalte, die Solidarität zu organisieren und die einzubeziehenden Gruppen der Größe und der Art nach zu bestimmen. Hier hat der Gesetzgeber drei Optionen:

- den Kreis der Zwangsversicherten auf alle auszudehnen und eine Volksversicherung einzurichten,

- am heutigen Versichertenkreis festzuhalten oder

- den Kreis (mit den vorstehend beschriebenen Folgen) einzuschränken.

Von den drei Optionen erscheint das Festhalten am Status quo am wahrscheinlichsten, verbunden mit Variationen der Beitragsgestaltung und Finanzierung (vgl. Arbeitsgruppe 1). Unverzichtbar erscheint eine Überprüfung des Leistungskataloges, der auf einen Kernbereich an notwendiger und ausreichender Versorgung zurückzuführen sei, ohne daß das Solidaritätsprinzip gefährdet werde. Im Gegenteil ist es nur durch eine Leistungsbeschränkung im obengenannten Sinne zu sichern.

\section{Korrektur des Leistungskataloges auf der Makroebene}

Das Solidaritätsprinzip wird nicht verletzt, wenn die Leistungen des GKV-Systems nach Art und Menge für alle Versicherten in gleicher Weise reduziert und daher nicht einzelne Gruppen von Versicherten von Leistungen ausgeschlossen werden. Die Frage ist, ob es die Möglichkeit einer „kollektiven Leistungsbeschränkung" gibt.

\subsection{Versicherungsfremde Leistungen}

Schon lange wird in der öffentlichen Diskussion der Ausschluß sog. versicherungsfremder Leistungen aus der GKV gefordert. Sie haben mit deren primärer Zielsetzung, nämlich eine Versorgung im Krankheitsfall zu sichern, nichts zu tun. Sie sind aber als Leistungen politisch gewollt, sie sind sozial gerechtfertigt und nur aus pragmatischen Gründen der GKV zur Finanzierung zuge- 
schoben worden. Dazu zählen u. a. die beitragsfreie Mitversicherung von einkommenslosen Familienangehörigen, der Mutterschutz, die Schwangerschaftsverhütung und -unterbrechung, Kuren, die Versorgung mit Sehhilfen, die sich schon deswegen nicht als Versicherungsleistung eignen, weil sie absehbar praktisch von jedermann spätestens ab dem 50 . Lebensjahr benötigt werden. Ähnlich ist ein Großteil der Zahnprothetik einzuschätzen. Eine partielle Ausgrenzung ist hier wie bei Brillen und Hörhilfen durch Zuschußregeln verwirklicht worden.

Der im Rahmen der GKV realisierte Familienlastenausgleich ist sozialpolitisch sinnvoll und sollte aufrechterhalten werden, zumal die Familien sowohl bei der Steuer als auch bei der Alterssicherung gegenüber den Kinderlosen benachteiligt sind. Die Frage ist aber, warum ein sozialpolitisch notwendiger und erwünschter Schutz nur einem Teil der Bevölkerung als Umverteilungslast aufgebürdet wird. Der Schutz der Familie ist eine allgemeine sozialpolitische Aufgabe und müßte daher über die Steuer finanziert werden.

Der defizitären Staatsfinanzen wegen besteht zwar derzeit keinerlei Aussicht, die Überwälzung dieser Staatsaufgabe auf die GKV rückgängig zu machen, doch waren sich alle Teilnehmer an der Diskussion einig, dies zu empfehlen. Offen blieb, in welcher Form die staatliche Finanzierung erfolgen sollte: ob als Zuschuß an die einzelnen Empfänger oder als Pauschale an die GKV. Aus pragmatischen Gründen sprachen sich die meisten Mitglieder der Arbeitsgruppe für einen formellen Verbleib des Familienlastenausgleichs in der GKV aus. Man war sich darüber einig, daß das Problem von seiner finanziellen Größenordnung her nicht die gleiche Bedeutung hat wie etwa die versicherungsfremden Leistungen in der gesetzlichen Rentenversicherung.

Die vergleichsweise geringe finanzielle Bedeutung bestimmte die Art der Empfehlungen zum Umgang mit den anderen versicherungsfremden Leistungen in der GKV. In erster Linie wurde es aus sozialpsychologischen Gründen als wichtig angesehen, Leistungseinschränkungen vorzunehmen: Es kann so der Illusion vorgebeugt werden, die GKV sei eine Versicherung gegen alle auch selbstverschuldete - Risiken des Lebens, die bequemerweise von einer möglichst großen Versichertengemeinschaft zu tragen seien. Welche versicherungsfremden Leistungen entweder zu reprivatisieren oder auf die Gemeinschaft der Steuerzahler zu 
übertragen sei, richte sich mehr nach der aktuellen Durchsetzbarkeit als nach einer jedem einsichtigen Sachrationalität. Mit den Leistungsreduktionen bei Brillen, beim Zahnersatz und bei Kuren sei ein richtiger Weg eingeschlagen worden. Als Beispiele besonders provozierender Inanspruchnahmen von solidarischem Versicherungsschutz wurden die Risiken überdurchschnittlich unfallträchtiger Sportarten oder von Abenteuerreisen genannt, für die von den einzelnen Versicherten privat hohe Summen aufgewandt werden, hingegen das Erkrankungsrisiko auf die Versichertengemeinschaft abgewälzt wird. Hier seien entweder Leistungsausschlüsse oder Zuschläge zu den Versicherungsbeiträgen angebracht.

\subsection{Unwirksame und schädliche Leistungen}

Ähnlich wie die zuletzt genannten Leistungen könnten auch jene behandelt werden, die zwar auf die Versorgung im Krankheitsfall ausgerichtet sind, aber einem strengen Maßstab des "Notwendigen" nicht genügen, weil entweder ihre Wirksamkeit auch beim Anlegen wenig strenger Kriterien nicht plausibel gemacht werden kann oder gar - wie etwa bei der hyperbaren Sauerstofftherapie des Tinnitus - damit Gefährdungen und Risiken verbunden sind, denen kein Nutzen gegenübersteht. Der Sachverständigenrat für die konzertierte Aktion im Gesundheitswesen hat in seinem Gutachten 1996 eine ganze Reihe von einschlägigen Leistungen aufgezählt. Unter innen ist die in der Mehrzahl der Fälle sinnlose Knochendichtemessung bei der Osteoporose ein bekanntes Beispiel. Zweifelhaft sind auch einige Bluthochdrucktherapien oder die Normalisierung eines rechnerisch zu hohen Cholesterinspiegels im Rahmen der Primärprävention.

Die Gruppe war der Meinung, daß es sich durchaus lohnt, einer Ausuferung des Leistungskataloges durch den Ausschluß von Leistungen mit nicht belegtem Nutzen Grenzen zu setzen, auch wenn man davon wegen der Größenordnung der hierauf entfallenden Vergütungen keine Sanierung des finanziellen Defizits der GKV erwarten dürfe. In diesem Zusammenhang wurde von einigen Teilnehmern Unverständnis gegenüber dem Bundesgesundheitsminister geäußert, der die Positivliste aus seinem Reformkatalog gestrichen hat. Die Schwierigkeiten bei der Erstellung einer solchen Liste wurden allerdings nicht übersehen, wobei die Einschränkung der ärztlichen Therapiefreiheit ebenso eine Rolle spielt wie der Eingriff der öffentlich-rechtlichen Institution GKV in 
den eingerichteten Gewerbebetrieb etwa eines Pharmaherstellers. Die rechtlichen Auseinandersetzungen um den Arzneimittelreport im November und Dezember 1997 haben gezeigt, welch hohe Anforderungen die Gerichte an den Nachweis der Unwirksamkeit von umstrittenen Medikamenten stellen, wenn zu befürchten steht, daß damit die Wettbewerbsstellung eines Pharmaunternehmens beeinträchtigt werden könnte.

\subsection{Einfluß der Sozialgerichtsbarkeit}

Die Haltung der Sozialgerichte bei der Interpretation dessen, was im Einzelfall als medizinisch notwendig und sinnvoll angesehen wird und damit von GK-Versicherten rechtlich beansprucht werden kann, wurde ebenfalls als eine über Jahrzehnte wirkende Ursache der Ausweitung des Leistungskataloges angesehen. Eine große Rolle kommt dabei der an einer Ausweitung neuer Leistungen und damit an einer Bestätigung ihres Fachs interessierten Gutachterschar zu. Bei dieser Kritik wurde das Dilemma nicht übersehen, das zwischen der Gewährung sozialer Gerechtigkeit im Einzelfall und der Effizienz des Gesamtsystems entstehen kann, wie sich dies am Beispiel der Zahlungspflicht von Krankenversicherungen für bestimmte homöopathische oder anthroposophische Heilverfahren bei der Behandlung schulmedizinisch "austherapierter" Patienten mit malignen Tumoren demonstrieren läßt. Die Arbeitsgruppe war sich zwar einig, daß der Gesetzgeber die damit verbundene Ausweitung der Inanspruchnahme von Leistungen durch geeignete Vorschriften eingrenzen sollte; der Weg dazu blieb jedoch undiskutiert.

\subsection{Die Rolle des Gesetzgebers}

Besonders heftig wurde darüber gestritten, welche Rolle der Staat und insbesondere der Gesetzgeber bei der Bereinigung des Leistungskataloges der GKV spielen sollte. Hier standen sich zwei Meinungen gegenüber: Die einen wollten den Gesetzgeber verpflichten offenzulegen, welche Leistungen im einzelnen aus dem Katalog der GKV herausgenommen werden sollen, um vor allem den Ärzten eine Entlastung gegenüber ihren Patienten zu verschaffen. Die anderen plädierten dafür, die Entscheidungen über das Notwendige und Nichtnotwendige den Ärzten zu überlassen und lieber deren Ermessensspielraum gesetzlich besser abzusichern und sie gegen Einsprüche gegen die Verweigerung von Leistungen zu schützen. Man einigte sich schließlich auf die 
Empfehlung, daß dann, wenn aus einem Mangel an Mitteln Leistungsausgrenzungen vorgenommen werden müssen, diese vom Gesetzgeber zu vertreten seien, um das Arzt-Patienten-Verhältnis nicht durch problematische ethische Entscheidungen zu belasten.

Bei Leistungsausgrenzungen durch den Gesetzgeber sollten aus ethischen Gründen folgende Regeln beachtet werden:

Es sollten keine Altersausgrenzungen für die Inanspruchnahme von Leistungen eingeführt werden, die es formell - entgegen oft geäußerter Meinung - auch nicht in Großbritannien gibt. Nicht das kalendarische Alter sei entscheidend für die Gewährung einer Leistung, sondern die Frage, ob ein Patient von einer medizinischen Leistung noch einen nachweisbaren medizinischen Nutzen, d. h. einen Nutzen für seine Lebensdauer und seine Lebensqualität habe. Würde im übrigen dieses Prinzip in der täglichen Praxis ernster genommen, dann könnten viele Leistungen in Diagnose und Therapie entfallen, ohne daß ethische Grundregeln verletzt würden.

Trotz einer nachhaltigen Betonung von mehr Eigenverantwortung sprach sich die Runde auch dagegen aus, das Verschuldensprinzip für den Ausschluß von Leistungen anzuwenden. Dies hat seinen Grund z. B. in den Schwierigkeiten, das Prinzip ohne Verletzung des Selbstbestimmungsrechtes durchzusetzen, einmal abgesehen von den Schwierigkeiten, im Einzelfall die Ursache einer Krankheit auf selbstverschuldetes Verhalten zurückzuführen. Unproblematisch könnten die schon erwähnten erhöhten Risiken bei gefährlichen Sportarten, etwa durch ausübungsgebundene Zuschläge zum Beitrag, abgesichert werden. Bei den zahlenmäßig im Vordergrund stehenden selbst verursachten Schädigungen durch Rauchen und Alkoholabusus haben Gesundheitsökonomen sogar nachgewiesen, daß diese Gruppe in der Gesamtbilanz weniger Leistungen in Anspruch nimmt als vergleichbare, nicht sichtbar sich selbst schädigende Kohorten. Diese Kranken sterben oft früh nach einem in der Regel kurzen Krankheitsverlauf und damit ohne große Aufwendungen. Außerdem nehmen sie überhaupt nicht oder nur kurz Renten in Anspruch. Gravierend ist darüber hinaus der grundsätzliche Einbruch in den Gedanken der Solidarität: So wie man einen Alkoholiker von der Leistung der solidarischen Versicherung ausschließt, weil er selbst an seiner Behandlungsbedürftigkeit schuldig sei, könnte man auch gegen die Eltern behinderter Kinder oder gegen 
behinderte Alte geltend machen, daß sie es in der Hand gehabt hätten, durch geeignete Maßnahmen wie Schwangerschaftsverhütung oder Nichtinanspruchnahme lebensverlängernder medizinischer Maßnahmen wie z. B. der Dialyse zu verhindern, daß die Versichertengemeinschaft für die Behandlung bezahlen müsse.

Zusammenfassend können von einer „positivistischen“ Begrenzung des Leistungskataloges zwar wichtige psychologische Signale ausgehen, aber davon kann keine durchgreifende Sanierung der GKV-Finanzen erwartet werden. Dies wird durch die bekannte, in der Diskussion am Rande erwähnte Tatsache bestätigt, daß bisher alle Versuche fehlgeschlagen sind, den Leistungskatalog unter strenger Auslegung des medizinisch Notwendigen durch objektivierende Abwägungen und Vereinbarungen ex ante und für alle Versicherten gleich wirksam einzuschränken. Zwei Gründe sind dafür maßgeblich: Der erste liegt im Egoismus der medizinischen Fachdisziplinen, der es unmöglich macht, Vereinbarungen zu treffen, die von den einzelnen Disziplinen Selbstbeschränkungen verlangen, mit denen ein Bedeutungsverlust verbunden ist. Der zweite liegt in der Erkenntnis, daß das, was beim einen Patienten bei gleicher Diagnose sinnvoll ist, bei einem anderen Patienten medizinisch überflüssig oder aber zu wenig sein kann. Es kommt eben nicht auf die begrifflich fixierte Diagnose, sondern auf die nicht standardisierbare Indikation bei einer Diagnose an.

\section{Beeinflussung der Leistungserstellung auf der Mikroebene}

Die Arbeitsgruppe hat unter Berücksichtigung der vorstehend beschriebenen Schwierigkeiten die Betonung auf Instrumente gelegt, die nicht generell einzelne Leistungen ausgrenzen, sondern eine Beschränkung bei der Leistungserstellung und der Leistungsprozesse im konkreten Fall zum Ziel haben. Drei dafür geeignete Ansatzpunkte wurden diskutiert:

- die Verschärfung der Indikationsstellung,

- vertragliche Vereinbarungen zwischen Leistungserstellern und Kostenträgern über die Leistungsprozesse und deren wirtschaftliche Erstellung,

- neue Organisationsformen der Solidarität auf der Beitragsebene. 


\subsection{Verbesserung der Indikationsstellung}

Die Verschärfung der Indikationsstellung geht von der von allen Medizinern zugegebenen Tatsache aus, daß beim Anlegen strenger Maßstäbe bei einem großen Teil der Patienten die Indikation zu Leistungen nicht gegeben ist. Von Sachkennern werden Zahlen zwischen $20 \%$ und $50 \%$ genannt. Die Gründe sind:

- der Mangel an verbindlichen Leitlinien zur Interpretation von medizinischem Wissen und medizinischen Befunden,

- der Erwartungsdruck von Patienten, daß etwas unternommen werden müsse,

- die mangelhafte Ausbildung und Übung in der "sprechenden" Medizin,

- die Einkommensinteressen der Leistungserbringer, die im gegenwärtigen System durch aktive, intervenierende medizinische Maßnahmen, nicht aber durch Beratung und schon gar nicht durch Abraten oder Nichterstellen vor allem von somatisch orientierten Interventionsleistungen befriedigt werden.

Für eine Änderung oder Verbesserung müßte eine neue Kultur der medizinischen Leistungserbringung entstehen, die das wie unscharf auch immer zu definierende, wirklich Notwendige und nicht in erster Linie das Mögliche in den Mittelpunkt stellt. Es darf nicht nach der Wirkung auf einen medizinischen (oft Surrogat-) Parameter, sondern es muß nach dem Nutzen gefragt werden, den der Patient von einer Maßnahme hat. Dies wäre keineswegs nur aus Gründen der Ressourcenschonung, sondern aus ethischen Gründen geboten: Jede medizinische Intervention birgt auch iatrogene Risiken, denen im Falle unnötiger Interventionen kein Nutzen für den Patienten gegenübersteht.

Die Kriterien für eine strengere Indikationsstellung sind bekannt:

- die Überflüssigkeit einer medizinischen Intervention diagnostischer oder therapeutischer Art,

- die Folgenlosigkeit einer diagnostischen und die Wirkungslosigkeit einer therapeutischen Maßnahme, 
- die Absehbarkeit eines Krankheitsverlaufs mit hoher SpontanHeilungswahrscheinlichkeit oder mit sicherem infausten Ausgang,

- die mangelnde Effizienz von medizinischen Maßnahmen, d. h., wenn ein sehr hoher Aufwand für vergleichsweise geringe Wirkungen und geringfügigen Nutzen zu leisten ist.

Diese Ziele können nur unter bestimmten Rahmenbedingungen erreicht werden:

- Bereits während der Ausbildung muß eine kritische Haltung gegenüber technischen Leistungen anerzogen werden.

- Es muß die Fähigkeit vermittelt werden, den Patienten vom Sinn einer somatischen "Nichtintervention“ zu überzeugen und eine Alternative anzubieten, die dem Patienten hilft, seine Beschwerden zu bewältigen.

- Die Entscheidung, ob im einen Fall etwas notwendig, im anderen Fall überflüssig ist, wird oft dadurch erschwert, daß es keine wissenschaftlich konsensfähigen Regeln gibt. Insofern kann sich die "Beliebigkeit" sogar sachlich erklären. Der Umgang mit diesem Problem muß ebenfalls Gegenstand der Aus-, Fort- und Weiterbildung werden.

- Die Rechtsprechung zur Haftung bevorzugt in der Tendenz eher das Handeln als das Unterlassen. Ein wünschenswertes Umdenken müßte von anerkannten Experten gestützt und nicht durch überzogene Gutachten verhindert werden.

- Das Honorierungssystem ist so zu reformieren, daß die Erbringung der Einzelleistung im Sinne einer somatisch orientierten, medizinisch notwendigen Intervention ebensowenig bestraft wird wie die begründete "Nichtintervention“.

Von den in der Diskussion behandelten Rahmenbedingungen sind diejenigen am schnellsten den genannten Zielen anzupassen, die es mit der Zuverlässigkeit und Verbindlichkeit medizinischer Erkenntnis zu tun haben. Dazu zählt die Entwicklung von Leitlinien, die im Zusammenhang mit der Qualitätssicherung wesentlich dazu beitragen können, das Leistungsgeschehen auf das tendenziell Notwendige zu beschränken. Dies besonders dann, wenn sie nicht bloß durch eine Addition aller Anforderungen aus 
den verschiedenen Fächern zustande kommen und wenn sie als verbindlich angesehen werden. Ärztekammern und Fachgesellschaften haben hier wesentliche Aufgaben zu lösen. Ein anderer Ansatzpunkt ist die "evidence based medicine", die sich auf das Gesicherte verständigt, d. h. auf das, dessen Wirksamkeit durch methodisch sichere Studien belegt ist, ohne deshalb zur Kochbuchmedizin zu werden. Damit dürften in Deutschland, insbesondere bei den kostenträchtigen Behandlungen, höchst erwünschte Folgen verbunden sein.

\subsection{Vertragsvereinbarungen zur Optimierung der Allokation}

Die Zurückhaltung bei der Indikation einzelner medizinischer Interventionen ist nur dann ein Ansatz zur Reduktion der Leistungen und damit der Kosten, wenn die eingesparten Ressourcen weil sie nun einmal in Form von Personal und Einrichtungen vorhanden sind - nicht an anderer Stelle zu ungerechtfertigten Leistungsvermehrungen führen. Die Verlagerung der bisher stationären Herzkatheter-Untersuchungen in den ambulanten Bereich mit der Folge eines Anstiegs der Leistungen um mehr als $200 \%$ ist ein Beispiel dafür, was gemeint ist.

Solche Entwicklungen können durch Ansätze von Managed Care unterbunden werden, besonders, wenn sich die Leistungsersteller und die Kosten- und Finanzierungsträger zusammentun, um optimale Behandlungsformen und deren Erstellung in optimalen Prozessen zu organisieren. Dieser umfassende Ansatz zeigt in den USA beachtliche Erfolge, auch wenn dabei nicht übersehen werden sollte, daß zum Teil Lasten von einem Bereich des Gesundheitswesens in einen anderen oder in den privaten Bereich hinein verschoben werden. Für die europäischen Verhältnisse dürtten die Versuche in der Schweiz als Anregung, weniger als unmittelbar übertragbares Vorbild dienen. Leicht werden die dortigen historischen Rahmenbedingungen bei ihrer Einführung unterschätzt und unter Berücksichtigung der in den USA erzielten Ergebnisse die bisherigen Erfolge überschätzt. Aber sie sind ein Beispiel dafür, daß etwas auch unter den Bedingungen europäischer Sozialsysteme verändert werden kann. In der Bundesrepublik Deutschland haben immerhin erste Versuche begonnen, mit dem Disease Management bei bestimmten Krankheiten zu einer rationaleren Medizin zu kommen. 


\subsection{Neue Organisationsformen der Solidarität}

Nach aller Erfahrung ist der Gesetzgeber in einer parlamentarischen Demokratie mit dem dort starken Einfluß der in Verbänden organisierten Interessen nicht in der Lage, den differenzierten Präferenz- und Steuerungsbedürfnissen im Gesundheitswesen im Rahmen öffentlich-rechtlicher Gestaltungsregeln gerecht zu werden. Es wurden daher zwei weitere Vorschläge zur Debatte gestellt, ohne daß diese mit einem Votum abgeschlossen werden konnten.

Der erste Vorschlag griff den Hinweis aus der Eingangsdiskussion wieder auf, wonach das Solidaritätsprinzip zwar eine Umverteilung von oben nach unten beinhalte, aber keineswegs eine solche von unten nach oben, wie sie gegenwärtig existiere und weiter vorne dargestellt wurde. Mit wachsendem individuellem Einkommen müßten daher entweder wachsende und am individuellen Gesamteinkommen orientierte Beiträge entrichtet oder aber wie das mit der jüngsten Gesetzgebung durch Erhöhung der Eigenleistungen realisiert wird - im Krankheitsfall entsprechend differenzierte Zuzahlungen fällig werden, so daß der Anteil der solidarischen Finanzierung sinkt. Dies sollte mit einem verstärkten Wettbewerb der Kassen verbunden werden, der zu einer präferenzadäquateren Leistungsdifferenzierung führt. Wenn dies politisch nicht durchsetzbar ist, müßte mindestens die Beitragsbemessungsgrenze dynamisch gehalten werden, um wenigstens den medizinischen Fortschritt ausreichend zu sichern.

Der zweite Vorschlag - in einem "Impulsreferat" vorgestellt - sieht die Gründung eines Krankenversicherungsfonds vor, in den alle Bürger einkommensabhängig gestufte Beiträge einzahlen und dafür einen für alle Versicherten gleichwertigen Leistungsgutschein erhalten, mit dem sie bei konkurrierenden Krankenversicherungen differenzierten Versicherungsschutz einkaufen können, dessen Umfang in einem Kernbereich hoheitlich überwacht werden muß. Die Gefahr der Risikoselektion ist durch Kontrahierungszwang auszuschließen.

Soweit zu beiden Vorschlägen innerhalb der beschränkten Zeit noch eine Diskussion geführt werden konnte, gehen beim ersten Vorschlag die Bedenken dahin, daß es zu einer Umwandlung in ein Volksversicherungssystem kommen könnte: Es wäre nicht einzusehen, ausgerechnet die Bezieher der höchsten Einkommen 
aus der Solidarhaftung zu entlassen. Das System könnte allerdings auch so gestaltet werden, daß mit einem einkommensabhängigen Pflichtbeitrag - wie bei der gesetzlichen Alters- und Hinterbliebenenversorgung in der Schweiz - der Solidaranspruch befriedigt wird, mit dem dann ein für alle gleicher, relativ niedriger, aber das Notwendige abdeckender Versorgungsanspruch korrespondieren würde, so daß sich jeder nach eigenen Präferenzen zusätzlich versichern kann. Es blieben aber viele Fragen offen.

Der zweite Vorschlag bezieht alle Bürger in die Solidarität ein und gewährt eine Kernversorgung mit der Möglichkeit differenzierter Zusatzversicherung. Offen bleibt aber, was mit den gesetzlichen Krankenkassen in diesem System und ihrem öffentlichrechtlichen Charakter geschehen soll: Für den „Verkauf" von Versicherungsleistungen in Konkurrenz untereinander und zu den privaten Krankenversicherern ist keine öffentlich-rechtliche Organisationsform notwendig, wenn es einen Kontrahierungszwang und eine Versorgungsaufsicht für einen Kernbereich für alle Versicherer gibt.

Die Diskussion zeigte, daß dieses Modell eine eigene und eingehendere Erörterung verdient.

\section{Zusammenfassung}

Faßt man das Ergebnis der Diskussion zusammen, dann erwiesen sich folgende Aussagen in der Arbeitsgruppe als konsensfähig und wurden als Empfehlungen an das Plenum weitergegeben:

Zur Sicherung einer ausreichenden Grundversorgung für zumindest die ärmere Bevölkerung ist eine Umverteilung von oben nach unten unverzichtbar. Das Beitragsaufkommen der ärmeren Bevölkerung reicht nicht aus, um eine effiziente Versorgung für diese Gruppe zu sichern.

Die Versuche, über eine Bereinigung des Leistungskataloges zu einer Ausgabenbeschränkung zu kommen, sind psychologisch als Signale gegen eine überbordende InanspruchnahmeMentalität wichtig, aber dem Umfang nach wenig effizient. Wegen des öffentlich-rechtlichen Gleichheitsgebotes müssen zudem die Präferenzen aller über einen Kamm geschoren werden. 
Die staatliche Steuerung des Leistungsgeschehens muß wegen dessen Gleichförmigkeit generell scheitern. Die Reformen müssen daher bei den Vertragspartnern selbst ansetzen. Diese müssen sich im einzelnen auf strengere Indikationsstellungen, auf „evidence based medicine“, auf Modelle des Managed Care und des Disease- bzw. Case Management verständigen.

Der besseren Anpassung des Leistungsgeschehens an die $\mathrm{Be}-$ dürfnisse der Patienten und an die Kompetenz der Anbieter muß eine Neuverteilung und Neudefinition der solidarischen Finanzierung entsprechen, bei der vor allem eine wirksame Umverteilung von oben nach unten geboten ist, die entweder bei der Beitragsgestaltung und/oder bei Zuzahlungen im Krankheitsfall ansetzen könnte und mit mehr Wettbewerb der Kassen verbunden werden müßte. Zuzahlungsregelungen verletzen allerdings die Solidarität zwischen Gesunden und Kranken erheblich, weil sie die Letzteren stärker belasten, ohne daß sich dies im Zeitverlauf ausgleichen muß. 


\section{Resümee: Stabilisierung der gesetzlichen Krankenversicherung mit Hilfe adaptiver Reformen}

Eberhard Wille

\section{Das Solidaritätsprinzip als gemeinsame Ausgangs- position}

Obgleich das allgemeine Tagungsthema "Reformoptionen im Gesundheitswesen" lautete, konzentrierten sich die Diskussionen um alternative Konzepte auf die GKV. Allerdings gehen von den meisten Reformvorschlägen im Bereich der GKV vielschichtige Effekte nicht nur auf die Situation der diversen Leistungserbringer, sondern auch auf die private Krankenversicherung (PKV) und die Selbstmedikation aus. Unabhängig von der philosophischen Fundierung und der sozialpolitischen Einbettung des Solidaritätsprinzips herrschte weitgehende Einigkeit über die weitere Geltung dieses Prinzips und seine Implikationen für die Finanzierung und den Leistungskatalog der GKV. Ein ähnlicher Konsens bestand hinsichtlich der Notwendigkeit, die Reformoptionen, die der Gesetzgeber mit den Strukturverträgen und Modellvorhaben eröffnete, zügig und intensiv zu nutzen. Am umstrittensten blieben die komparativen Vor- und Nachteile der zur Diskussion stehenden Finanzierungsoptionen. Dabei teilten allerdings nahezu alle Teilnehmer die Einschätzung, daß sich die Finanzierungsprobleme der GKV künftig im Zuge der wirtschaftlichen Entwicklung nicht von selbst abbauen und die Ausschöpfung des Rationalisierungspotentials nicht ausreicht, um im geltenden System Beitragssatzstabilität zu gewährleisten. Bei Übereinstimmung über die grundsätzliche Notwendigkeit von fiskalisch stabilisierenden Reformmaßnahmen divergierten vornehmlich bei den $\mathrm{Fi}$ nanzierungsoptionen die Vorstellungen über die adäquaten Alternativen.

Das Solidaritätsprinzip, an dem sich vor allem die Finanzierung der GKV orientiert, bietet in ökonomischer Hinsicht kein theoretisch fundiertes Paradigma, aus dessen Normen sich eindeutige Aussagen über die Beitragsgestaltung und die Abgrenzung des Leistungskataloges gewinnen lassen. Eine Solidargemeinschaft stellt auch eine private Krankenversicherung dar und ex post erfolgt auch hier immer eine Umverteilung von schadensfreien Mitgliedern zu solchen, die einen Schaden erlitten. Die darüber 
hinausgehende spezifische Solidarität im Rahmen der GKV besteht in einer Ex-ante-Umverteilung von:

- guten zu schlechten Gesundheitsrisiken (sog. Risikoausgleich),

- Beziehern höherer Arbeitsentgelte zu solchen mit niedrigeren Löhnen und Gehältern und zu den Arbeitslosen (Einkommensumverteilung),

- Alleinstehenden zu kinderreichen Familien (sog. Familienlastenausgleich) sowie

- jungen zu alten Versicherten (sog. Generationenausgleich).

Diese Verteilungswirkungen, die das Solidaritätsprinzip kasuistisch charakterisieren, standen bei den Teilnehmern in qualitativer Hinsicht nicht zur Disposition. Dies bedeutet eine Absage an eine Einengung der GKV auf sozial schwache Bürger im Sinne einer "Armenkasse“. Letztere setzt implizit entweder ganz oder teilweise eine Steuerfinanzierung voraus, denn die Mitglieder einer solchen Krankenkasse können mangels hinreichendem Einkommen für die von innen benötigten Gesundheitsleistungen nicht selbst im Sinne der gruppenmäßigen Äquivalenz aufkommen. Ein Festhalten an der bisherigen Ausprägung des Solidaritätsprinzips schließt neben einer steuerfinanzierten Gesundheitsversorgung auch prämienfinanzierte Systeme aus. Die GKV steht insofern ordnungspolitisch zwischen steuer- und prämienfinanzierter Gesundheitsversorgung. Dabei erfuhr das solidarische Element in der GKV durch den bundesweiten Risikostrukturausgleich (RSA) noch eine spürbare Aufwertung bzw. Intensivierung, denn er führte zu einer deutlichen Reduktion der Beitragssatzunterschiede zwischen den einzelnen Krankenkassen. Der RSA und die Beitragssatzkalkulation grenzen auf unterschiedlichen Ebenen verschiedene Solidargemeinschaften voneinander $a b$.

\section{Der Leistungskatalog im Licht des Solidarprinzips}

Die Einengung des Leistungskataloges bildet für die GKV insofern eine globale Finanzierungsoption, als die dadurch frei werdenden Mittel nun in andere, vor allem dringlichere Gesundheitsleistungen fließen können. Die Versicherten bzw. Patienten müssen in einem solchen Fall nicht zwangsläufig auf diese Gesundheitsleistungen verzichten, denn es steht innen frei, diese im Rahmen der Selbstmedikation zu erwerben oder eine entspre- 
chende private Zusatzversicherung abzuschließen. So induzierten z. B. die Einsparungen, die das Arzneimittelbudget im Jahre 1993 in der GKV verursachte, einen signifikanten Anstieg der Arzneimittelausgaben im Bereich der Selbstmedikation, und die Ausklammerung des Zahnersatzes für Jugendliche stimulierte in der PKV den Abschluß entsprechender Zusatzversicherungen. Die Versicherten gelangen ohne jede Minderung ihres verfügbaren Einkommens weiterhin in den Genuß von Gesundheitsleistungen, wenn deren Finanzierung lediglich von der GKV auf einen anderen Sozialversicherungsträger oder die öffentlichen $\mathrm{Ge}$ bietskörperschaften übergeht.

In diesem Zusammenhang steht die häufig erhobene Forderung, die krankenversicherungsfremden Leistungen der GKV aus anderen öffentlichen Budgets zu finanzieren. Die krankenversicherungsfremden Leistungen belaufen sich in enger Abgrenzung auf gut $4 \mathrm{Mrd}$. DM und schließen weiter gefaßt den gesamten sog. Familienlastenausgleich ein. Im Hinblick auf die Frage nach den Finanzierungsinstrumenten dieser Leistungen außerhalb der GKV bestehen zwischen den Arbeitsgruppen 1 und 3 enge thematische Beziehungen. So bietet sich z. B. zur Finanzierung dieser Leistungen aus den Budgets der Gebietskörperschaften neben Ausgabensenkungen auch eine Erhöhung des Mehrwertsteuersatzes an. Eine Mehrwertsteuererhöhung besitzt gegenüber vielen anderen Finanzierungsinstrumenten den Vorzug, als allgemeine Verbrauchersteuer nicht die Investitionen zu belasten und wegen des Bestimmungslandprinzips beim grenzüberschreitenden gewerblichen Handel in der Europäischen Union auch nicht die Exporte zu behindern bzw. die Importe zu fördern. Da es sich bei der familienpolitischen Förderung um eine gesamtgesellschaftliche Aufgabe handelt, deren Finanzierung nicht nur den Versicherten der GKV, sondern allen Bürgern obliegt, besitzt die Forderung nach Umfinanzierung dieser Leistungen unabhängig von den jeweiligen politischen Realisierungschancen sowohl unter Solidaritäts- als auch unter intertemporalen Äquivalenzaspekten ihre grundsätzliche Berechtigung. Dabei spielt es eine vergleichsweise untergeordnete Rolle, ob diese Leistungen dann auch organisatorisch auf eine Institution außerhalb der GKV übergehen oder diese die entsprechenden Aufwendungen lediglich aus den Budgets der Gebietskörperschaften erstattet erhält.

Abgesehen von krankenversicherungsfremden Leistungen, die an der (hier nicht vorliegenden) Morbidität anknüpfen, stellt sich bei 
einer Überprüfung des Leistungskataloges der GKV zunächst die Frage nach den Ausschlußkriterien. Eine Gesundheitsleistung gehört nicht bzw. um so weniger in den Leistungskatalog der GKV, wenn folgende Tatbestände vorliegen:

- Wirkungslosigkeit im Hinblick auf die medizinischen Outcomes,

- Ineffizienz der Gesundheitsproduktion, d. h. alternative Verfahren ermöglichen eine bessere Zielrealisierung oder verwirklichen die gleichen Outcomes mit geringeren Ressourcen,

- finanzielle Tragbarkeit bzw. geringe soziale Belastung,

- Vorhersehbarkeit des Schadensfalles,

- Vermeidbarkeit des Ereignisses sowie

- niedrige Kosteneffektivität.

Bei Vorliegen von einem der beiden ersten Kriterien handelt es sich eindeutig um eine Rationalisierung im Sinne des ökonomischen Effizienzpostulates. Letzteres zielt darauf ab, die Verschwendung knapper Ressourcen zu vermeiden und über die alternative Verwendung der eingesparten Mittel den Nutzen der Wirtschaftssubjekte in der Gesellschaft zu erhöhen. In einer Welt beschränkter Ressourcen gewinnt das Effizienzpostulat, obwohl es zunächst nur ein formales, wertfreies Prinzip darstellt, zumindest mittelbar auch eine ethische Dimension. Das Solidaritätsprinzip verlangt ebenfalls nach einer Ausschöpfung des Rationalisierungspotentials. Die Bereinigung des Leistungskataloges der GKV um wirkungslose Maßnahmen, z. B. über Positivlisten oder erweiterte Negativlisten, stößt allerdings in der Praxis häufig dadurch auf kaum überwindbare Probleme, als die meisten Leistungen nicht per se, sondern nur bei bestimmten Indikationen und/oder bestimmten Personengruppen wirkungslos bleiben. Das Effizienzproblem der deutschen Gesundheitsversorgung besteht weniger in einem zu umfangreichen Leistungskatalog als vielmehr in einem zu intensiven bzw. zuwenig selektiven Gebrauch desselben. Zudem können bei Gesundheitsleistungen die Wirksamkeit im Sinne einer Veränderung medizinischer Parameter und der therapeutische Nutzen bzw. die individuelle Wohlfahrt des Patienten deutlich voneinander abweichen.

Bei allen anderen Ausschlußkriterien (außer den beiden ersten) geht die Einengung des Leistungskataloges mit einer Rationierung einher, denn die Versicherten bzw. Patienten erhalten dann nicht den von ihnen gewünschten Umfang an - möglicherweise durchaus effizient produzierten - Gesundheitsleistungen. Eine 
Rationierung von Gesundheitsleistungen muß aber grundsätzlich weder mit dem Effizienzpostulat noch mit dem Solidaritätsprinzip in Konflikt geraten. Beide Prinzipien können aus gesamtwirtschaftlicher Perspektive einen Verzicht auf bestimmte Gesundheitsleistungen nahelegen, wenn der Nutzen, den diese Güter stiften, geringer als ihre Opportunitätskosten ausfällt. Dies bedeutet, daß die durch die betreffenden Gesundheitsleistungen gebundenen Ressourcen in einer anderen Verwendung einen höheren Nutzen erzeugen würden. Infolge der generellen Ressourcenknappheit steht jede medizinische Maßnahme sowohl mit anderen Projekten im Gesundheitswesen als auch mit Vorhaben in den übrigen wohlfahrtsrelevanten Lebensbereichen in Konkurrenz um die knappen Mittel. Eine Rationierung auch effizient produzierter Gesundheitsleistungen, die für ihre Empfänger einen therapeutischen Nutzen abwerfen, konfligiert insofern nicht zwangsläufig mit ethischen Werten bzw. dem Solidaritätsprinzip, denn die betreffende Ressourcenverlagerung, die einen Verzicht auf diese Leistungen impliziert, kann die gesellschaftliche Wohlfahrt erhöhen. Trotz der berechtigten Forderung „Rationalisierung vor Rationierung" wirft nicht jede Mengenrationierung im Gesundheitswesen ethische Probleme auf und mündet auch nicht notwendigerweise in eine "Zweiklassenmedizin".

Die Qualitätssicherung orientiert sich in instrumentaler Hinsicht ebenfalls am Effizienzpostulat, indem sie eine zügige und umfassende Diffusion und Umsetzung des vorhandenen indikationsspezifischen Wissens anstrebt. In diesem Zusammenhang können im Hinblick auf die Prozeßqualität evidenzbasierte Leitlinien bzw. Guidelines und - wo immer möglich - ergebnisorientierte Vergütungssysteme zum Einsatz kommen. Diese Guidelines mögen in manchen Fällen auch weniger Gesundheitsleistungen bzw. Mengenrationierungen nahelegen. Sie konfrontieren dann den einzelnen Arzt mit dem Compliance-Problem, dem Patienten die medizinische Berechtigung einer Unterlassung bzw. Nichtverordnung zu erklären, die häufig nicht mit dessen Erwartungshaltung konform geht. Im Konfliktfall bedürfen die Ärzte dann auch einer Unterstützung durch die Politik und eines gewissen Problemverständnisses seitens der Rechtsprechung. Andernfalls besteht die Gefahr, daß sie zu einer ausgabenintensiven Defensivmedizin Zuflucht nehmen. Insoweit Strukturverträge und Modellvorhaben solche Guidelines in Managed-Care- oder Disease-ManagementKonzepten verankern, überschneidet sich die Thematik der Arbeitsgruppen 2 und 3 (siehe unter 4 ). 


\section{Spezielle Finanzierungsoptionen}

\section{a) Generelle Ansatzpunkte}

Im Rahmen der Beitragsgestaltung in der GKV können die diversen Finanzierungsoptionen im konkreten Fall an

- der GKV-Versicherungspflicht bzw. dem Pflichtversichertenkreis,

- der Beitragsbemessungsgrenze,

- der Beitragsbemessungsgrundlage,

- der beitragsfreien Mitversicherung und/oder

- der aufkommensneutralen Änderung der Beitragssätze bzw. Beitragssatzanteile

ansetzen. Mit Ausnahme der letzten aufkommensneutralen $\mathrm{Fi}$ nanzierungsoption zielen alle anderen derzeit diskutierten Alternativen auf Mehreinnahmen für die GKV ab.

\section{b) Anhebung von Pflichtversicherungs- und Beitragsbemessungsgrenze}

Um den Pflichtversichertenkreis in der GKV auszuweiten, existieren grundsätzlich folgende Möglichkeiten:

- Anhebung der Versicherungspflichtgrenze auf das Niveau der Beitragsbemessungsgrenze der GRV, d. h. von derzeit (1998) 6.300 DM auf 8.400 DM bzw. in den neuen Bundesländern von 5.250 DM auf 7.000 DM pro Monat,

- Aufhebung der Versicherungspflichtgrenze oder

- Einbeziehung der gesamten Bevölkerung, d. h. auch aller Selbständigen und Beamten, in die GKV.

In der angegebenen Reihenfolge erweitern diese Varianten den Pflichtversichertenkreis bis zur allgemeinen Volksversicherung. Die Ausweitung des Pflichtversichertenkreises kann grundsätzlich bei

- geltender Beitragsbemessungsgrenze

- einer Anhebung der Beitragsbemessungsgrenze auf das Niveau der GRV oder 
erfolgen. Bei den möglichen Kombinationen aus GKV-Versicherungspflicht und Beitragsbemessungsgrenze erscheinen allerdings nur jene Varianten sinnvoll, in denen die Beitragsbemessungsgrenze nicht über der Versicherungspflichtgrenze liegt. Andernfalls droht bei einer Anhebung der Beitragsbemessungsgrenze eine Abwanderung des betroffenen Versichertensegments in die PKV.

Im Gegensatz zur gesetzlichen Renten- und Arbeitslosenversicherung, die unabhängig von irgendwelchen Einkommensgrenzen alle Arbeitnehmer einschließen, weist die GKV eine Pflichtversicherungs- und Beitragsbemessungsgrenze in Höhe von $75 \%$ des Niveaus der Beitragsbemessungsgrenze der GRV auf. Diese sog. Friedensgrenze, die über den Pflichtversichertenkreis auch das potentielle Marktsegment der privaten Krankenversicherung (PKV) absteckt, läßt sich nicht aus gesamtwirtschaftlichen Zielen ableiten bzw. sachlogisch begründen. Die Pflichtversicherungsgrenze erscheint im Licht des Solidaritätsprinzips zu niedrig und unter Subsidiaritätsaspekten zu hoch. So gibt es z. B. in den ebenfalls beitragsfinanzierten Systemen in Belgien und Frankreich keine Versicherungspflichtgrenze, sondern neben Sondersystemen für Beamte, Bergleute und Staatsbedienstete eine generelle gesetzliche Versicherungspflicht, so daß sich das Tätigkeitsfeld der privaten Versicherungen auf Zusatzleistungen beschränkt. In den Niederlanden dagegen liegt die Pflichtversicherungsgrenze deutlich niedriger als in Deutschland und die Arbeitnehmer besitzen im Fall einer Überschreitung dieser Grenze nicht mehr die Option, in der gesetzlichen Versicherung zu bleiben, d. h. analog zu Deutschland zwischen GKV und PKV zu wählen. Angesichts dieser normativen wie empirischen Beliebigkeit wundert es nicht, daß die geltende Versicherungspflicht- und Beitragsbemessungsgrenze von Zeit zu Zeit einen Gegenstand kontroverser Diskussionen bildet.

Von den zahlreichen Kombinationsmöglichkeiten, die zwischen einer Ausweitung des Pflichtversichertenkreises und einer Anhebung der Beitragsbemessungsgrenze bestehen, konzentrierte sich die Diskussion auf die Erhöhung von Versicherungspflichtund Beitragsbemessungsgrenze auf das Niveau der Beitragsbemessungsgrenze in der GRV. Dieser Vorschlag findet sich $u$. a. auch im zweiten Gesetzentwurf der Sozialdemokraten für die 
dritte Stufe der Gesundheitsreform. Die jeweils aufgelisteten Argumente gelten dann für weitergehende Vorhaben, wie $\mathbf{z}$. B. eine Aufhebung dieser Grenzen, tendenziell in verstärktem Maße.

In ihrer Modellrechnung für die GKV in den alten Bundesländern schätzte Frau Pfaff den fiskalischen Nettoeffekt dieser Finanzierungsoption auf 0,67 Prozentpunkte, d. h., daß eine Anhebung der Beitragsbemessungsgrenze der GKV auf das Niveau der GRV eine Senkung des Beitragssatzes um 0,67 Prozentpunkte erlauben würde. Räumt man den bisher privat Versicherten jedoch ein Wahlrecht ein, wofür verfassungsrechtliche Gründe sprechen, und macht die Hälfte der Betroffenen von diesem Bleiberecht Gebrauch, so dürfte sich die Beitragssatzsenkung auf ca. 0,4 bis 0,5 Prozentpunkte reduzieren, was einer absoluten Nettoentlastung von etwa $8 \mathrm{Mrd}$. DM entspricht.

Unabhängig von diesen intendierten fiskalischen Effekten sprechen vor allem folgende Argumente für eine solche Anhebung der Beitragsbemessungsgrenze:

- Die durch die Beitragsbemessungsgrenze erzeugte Tarifdegression verschiebt sich nach hinten, was innerhalb der Arbeitnehmer mit redistributiven bzw. positiven Verteilungseffekten einhergeht.

- Da die Gesamtbelastung zum Zeitpunkt der Reform gleich bleibt, erlaubt die Anhebung der Beitragsbemessungsgrenze eine Beitragsentlastung bei den mittleren und niedrigeren Arbeitsentgelten. Damit sinken die Arbeitskosten bei den weniger qualifizierten Arbeitskräften, die einen überdurchschnittlichen Anteil an Arbeitslosen aufweisen und beschäftigungspolitische Anreize besonders benötigen.

Diesen positiven Effekten stehen aber auch einige eher nachteilige Wirkungen gegenüber:

- Diejenigen Versicherten, die als schlechte Risiken (wieder) Zutritt zur GKV erhalten, ziehen daraus vor allem aus intertemporaler Sicht ungerechtfertigte Vorteile.

- Die höheren Beitragszahlungen wirken für die Betroffenen wie eine zusätzliche Steuerbelastung, denn diese kommen dadurch mit Ausnahme des Krankengeldes nicht in den Genuß von 
versicherungsmäßigen Mehrleistungen. Die Beiträge zur GKV verlieren auf diese Weise ihren ohnehin nur noch schwach ausgeprägten Versicherungscharakter, und der Tarif nähert sich dem einer linearen Lohnsteuer. Zudem trifft die zusätzliche Beitragsbelastung jene Versicherten, die in Verbindung mit dem geltenden Einkommenssteuertarif einen Grenzabgabensatz aufweisen, der schon derzeit deutlich über $50 \%$ liegt. Der Vorschlag von Frau Walzik, nur die Pflichtversicherungs-, aber nicht die Beitragsbemessungsgrenze anzuheben, vermeidet diesen Nachteil, was allerdings teilweise zu Lasten der anvisierten Mehreinnahmen geht.

- Die Anhebung der Beitragsbemessungsgrenze in der GKV auf das Niveau der GRV vereinheitlicht den Tarif von zwei Teilsystemen der sozialen Sicherung, die sich anders als nach ihrer Gründung zwischenzeitlich an unterschiedlichen Finanzierungsprinzipien ausrichten. Die GRV folgt ähnlich wie die Arbeitslosenversicherung tendenziell insofern immer noch dem Äquivalenzprinzip, als höhere Beitragszahlungen eines Versicherten zumindest bezogen auf seine Alterskohorte auch zu höheren Versicherungsleistungen berechtigen. Im Gegensatz dazu besitzen in der GKV alle Versicherten unabhängig von ihren Beitragszahlungen grundsätzlich denselben Anspruch auf medizinische Behandlungsleistungen.

- Eine isolierte Anhebung der Beitragsbemessungsgrenze verschärft im geltenden Beitragssystem, $d$. $h$. bei der bestehenden Bemessungsgrundlage, die Belastung der Bezieher von Arbeitsentgelten gegenüber den Beziehern anderer Einkunftsarten.

- Die PKV profitiert bei den Versicherten, die zur GKV überwechseln, zunächst von der mangelnden Portabilität der Altersrückstellungen, die ihr quasi als Umstellungsgewinne zufallen. Auf mittlere und längere Frist engt die Anhebung der Versicherungspflichtgrenze allerdings die Möglichkeiten der PKV ein, junge Versicherte zu gewinnen, und schwächt damit ihre finanzielle Grundlage.

- Privatversicherte Patienten zahlen vielfach für die gleichen Leistungen höhere Preise bzw. Gebühren als Patienten der GKV, so daß ein stärkerer Versichertenwechsel von der PKV zur GKV die Vergütung der Leistungserbringer spürbar vermindern kann. Es steht dann zu erwarten, daß die jeweiligen An- 
bieter versuchen, ihre Einbußen u. a. im Rahmen von Vergütungsverhandlungen zu kompensieren.

\section{c) Verbreiterung der Beitragsbemessungsgrundlage}

Die Verbreiterung der Beitragsbemessungsgrundlage kann im Prinzip über eine

- Erweiterung in Form einer Ergänzung des Arbeitsentgeltes durch weitere Einkunftsarten,

- Einbeziehung des Produktionsfaktors Kapital (sog. Maschinenbeitrag),

- Ausweitung auf die gesamte Wertschöpfung, d.h. auch auf Gewinne und Vermögenseinkommen, und/oder

- Ausdehnung auf Entgelte aus geringfügiger Beschäftigung

zu Mehreinnahmen in der GKV führen. Obgleich die jüngste politische Diskussion in diesem Kontext den Entgelten aus geringfügiger Beschäftigung eine besondere Beachtung schenkte, standen Pro und Contra einer Erweiterung der Beitragsbemessungsgrundlage um weitere Einkunftsarten im Mittelpunkt kontroverser Überlegungen.

Die Erweiterung der Beitragsbemessungsgrundlage könnte über die Arbeitsentgelte hinaus alle weiteren Einkünfte des Versicherten als beitragsrelevant heranziehen, $d$. h. neben den Einkünften aus nichtselbständiger Arbeit auch solche aus Land- und Forstwirtschaft, Gewerbebetrieb, selbständiger Arbeit, Kapitalvermögen, Vermietung und Verpachtung sowie sonstige Einkünfte umfassen. Eine erweiterte Bemessungsgrundlage würde dem Leistungsfähigkeitsprinzip eher entsprechen, das aber als alleiniges Beurteilungskriterium insofern zu kurz greift, als es dem Versicherungscharakter der GKV-Beiträge nicht Rechnung trägt und diese damit zu sehr in die Nähe der Einkommensbesteuerung rückt. Die Erweiterung der Bemessungsgrundlage schließt allerdings nicht aus, daß sich die Beitragsgestaltung durch die Beitragsbemessungsgrenze, den Tarif und das sog. Bruttoprinzip, das u. a. keine Verrechnung mit Verlusten aus anderen Einkunftsarten zuläßt, immer noch deutlich von der Einkommensbesteuerung unterscheidet. Bei einer erweiterten Bemessungsgrundlage würde sich die Beitragsgestaltung auch nicht weiter vom Äquivalenzprinzip 
entfernen, denn dieses differenziert - vom Krankengeld abgesehen - hier ebenfalls nicht nach Einkunftsarten.

Eine Erweiterung der Beitragsbemessungsgrundlage weist $u$. a. folgende Vorteile auf, wenn bei der konkreten Reformoption die Nicht-Arbeitsentgelte dem halben Beitragssatz unterliegen und ein gewisser Freibetrag oder eine entsprechende Freigrenze sog. Kleinsparer von der zusätzlichen Belastung freistellt:

- Die generelle Verbreiterung der Beitragsbemessungsgrundlage beseitigt die derzeitige auch verfassungsmäßig problematische Ungleichbehandlung von Pflicht- und freiwillig Versicherten.

- Es erfolgt eine stärkere Orientierung an der individuellen Leistungsfähigkeit, die in diesem Kontext zwar nicht das alleinige Kriterium, wohl aber eine Richtschnur für die Verteilungswirkungen bildet.

- Bei fehlender sozialer Schutzbedürttigkeit eines Mitglieds mit niedrigem Arbeitsentgelt stellt die Erweiterung der Beitragsbemessungsgrundlage auch einen Schritt in Richtung (zumutbarer) Risikoäquivalenz dar.

- Die Verbreiterung der Bemessungsgrundlage entlastet die Arbeitsentgelte bzw. Lohnnebenkosten und damit auch die Investitionen von Gesundheitsaufwendungen, die bei steigendem Rentneranteil immer stärker konsumtiven Charakter annehmen.

- Die erweiterte Bemessungsgrundlage schwächt die konjunkturelle Abhängigkeit der GKV-Einnahmen ab, da die NichtArbeitseinkommen in ihrer Summe vergleichsweise stetiger fließen.

- Angesichts des höheren Zinseinkommens älterer Menschen schwächt die generelle Einbeziehung dieser Einkunftsart in die Beitragsbemessung die intergenerative Umverteilung in der GKV ab. Dies erscheint vor dem Hintergrund einer erheblich gestiegenen Lebenserwartung und der sich abzeichnenden demographischen Entwicklung nicht nur berechtigt, sondern in Grenzen sogar geboten. Die gegebenen Systeme in der Sozialversicherung und in anderen Bereichen der öffentlichen Daseinsvorsorge drohen künftig die jeweils erwerbstätigen Generationen zu überfordern. 
Die Erweiterung der Beitragsbemessungsgrundlage um zusätzliche Einkunftsarten bringt aber auch Nachteile mit sich:

- Da die zusätzlichen Einkunftsarten nur unterhalb der Bemessungsgrenze Beitragsrelevanz besitzen, treten partiell regressive Verteilungseffekte auf.

- Durch die Einbeziehung weiterer Einkunftsarten gelangt ein Teil der bisher Pflichtversicherten über die Versicherungspflichtgrenze und erhält damit die Option, in die PKV abzuwandern. $\mathrm{Da}$ es sich bei den Abwandernden um gute Risiken handeln dürfte, entstehen der GKV daraus fiskalische Nachteile.

- Die Belastung der Zinseinkünfte könnte zusätzliche Anreize zur Kapitalflucht setzen mit entsprechend negativen Auswirkungen auf den inländischen Markt.

- Die Erhebungs- und Entrichtungsprobleme erscheinen vor allem bei den Zinseinkünften evident. Da der Risikostrukturausgleich Unterschiede in den Grundlohnsummen zwischen den Krankenkassen nivelliert, besitzen diese auch kaum ein Interesse daran, bei ihren Versicherten intensive Nachfragen bzw. Nachforschungen anzustellen.

- Mit der generellen Einbeziehung weiterer Einkunftsarten sinkt das relative Gewicht der Arbeitsentgelte und damit auch der Arbeitgeberbeiträge, was Konsequenzen bzw. Probleme hinsichtlich der paritätischen Besetzung der Selbstverwaltungsgremien aufwerfen könnte.

Bei einer zusammenfassenden Beurteilung von Pro und Contra dieser Reformoption gilt es zunächst zu berücksichtigen, daß die einzelnen 0. a. Aspekte nicht das gleiche Gewicht besitzen. So erscheint das im Prinzip zutreffende Argument einer drohenden Kapitalflucht in diesem Kontext häufig überschätzt, denn der halbe Beitragssatz für Zinseinkünfte und die Beitragsbemessungsgrenze limitieren hier im Gegensatz zur Einkommensbesteuerung die finanziellen Vorteile einer Kapitalflucht. Sodann existieren die Erhebungsprobleme schon heute bei den sog. Härtefällen sowie den freiwillig versicherten Rentnern, und sie entfallen auch künftig bei allen Versicherten, deren Arbeitsentgelt die Bemessungsgrenze erreicht. Obwohl angesichts der oben aufgelisteten Vorund Nachteile auch bei der Frage einer Erweiterung der Beitragsbemessungsgrundlage ein hinreichender Spielraum für persönli- 
che Werturteile verbleibt, sprechen per saldo allokative und distributive Aspekte eher für und technisch-organisatorische Gesichtspunkte eher gegen diese Reformoption.

\section{d) Einschränkung der beitragsfreien Mitversicherung}

Die beitragsfreie Mitversicherung von Familienangehörigen, die über kein eigenes versicherungspflichtiges Einkommen verfügen, ließe sich u. a.

- neben Kindern auf Ehepartner beschränken, die Kinder erziehen (schwächere Alternative: bzw. erzogen haben) oder Pflegedienste leisten,

- nur auf ein Kind einengen oder

- gänzlich aufheben.

Wie bereits oben unter 2 im Rahmen der krankenversicherungsfremden Leistungen aufgeführt, liefe eine völlige Aufhebung der beitragsfreien Mitversicherung von Familienangehörigen auf eine Suspendierung des sog. Familienlastenausgleichs innerhalb der GKV hinaus.

Von den drei angeführten Alternativen bezieht sich die Einschränkung der beitragsfreien Mitversicherung von Familienangehörigen als konkreter Reformvorschlag zumeist nur auf den nicht berufstätigen Ehepartner, der keine Kinder (mehr) erzieht und keine Pflegedienste leistet. Dabei könnte die Beitragsbelastung des nicht berufstätigen Ehepartners $u$. a. auf folgende Weise erfolgen:

- Beitragszahlung in Höhe von $50 \%$ des Beitrags des zugehörigen Mitglieds,

- Entrichtung eines (Mindest-)Beitrags entsprechend einem Arbeitsentgelt in Höhe der unteren Versicherungspflichtgrenze,

- Splitting des gemeinsamen Arbeitsentgeltes mit nachfolgender Anwendung des hälftigen Beitragssatzes auf beide Entgeltteile sowie

- Option, für eine der drei vorgenannten Varianten zu votieren.

Nach Schätzungen von Frau Pfaff würde die erste Variante bei einer gesonderten Beitragszahlung für mitversicherte Ehepartner 
ohne Kinder unter 13 bzw. unter 7 Jahren den Beitragssatz in den alten Bundesländern bei Aufkommensneutralität um 0,66 bzw. 0,88 Prozentpunkte absenken. Die Erhebung eines Mindestbeitrages erlaubt noch eine analoge Reduktion des Beitragssatzes um 0,4 Prozentpunkte. Im Unterschied zu diesen beiden Varianten führt das Splittingverfahren nur dann zu einer zusätzlichen Beitragsbelastung des jeweiligen Familienhaushaltes, wenn das Arbeitsentgelt des Mitglieds über der Beitragsbemessungsgrenze liegt. Andererseits belastet das Splittingverfahren im Vergleich zur geltenden Regelung auch zwei berufstätige Ehepartner stärker, sofern das Arbeitsentgelt des einen die Beitragsbemessungsgrenze unter- und das des Partners diese überschreitet. Ein Optionsrecht für eine der drei Varianten verursacht die geringste Beitragssatzsenkung, besitzt aber möglicherweise hinsichtlich seiner politischen Implementation Vorzüge. Die politische Akzeptanz würde wohl noch zunehmen und die fiskalische Ergiebigkeit vice versa abnehmen, wenn die beitragsfreie Mitversicherung von Familienangehörigen auch noch alle Partner einschließt, die einmal in der Vergangenheit Kinder erzogen oder Pflegedienste geleistet haben.

Unabhängig von einer Wahl zwischen diesen Varianten spricht grundsätzlich für diese Reformoption, daß das geltende Beitragssystem in dieser Hinsicht sowohl gegen das Äquivalenz- als auch gegen das Leistungsfähigkeitsprinzip verstößt. Die derzeitige Beitragsgestaltung benachteiligt offensichtlich Zweiverdienerfamilien, indem es sie bei gleicher oder sogar niedrigerer Summe der Arbeitsentgelte häufig stärker, im Grenzfall doppelt so stark belastet wie eine Familie mit einem erwerbstätigen Partner. Bei der Erhebung eines Mindestbeitrages und beim Splittingverfahren droht auch kaum eine Abwanderung von freiwillig Versicherten in die PKV, denn das Splittingverfahren führt nur dann zu einer zusätzlichen Beitragszahlung, wenn das Arbeitsentgelt des Mitglieds über der Beitragsbemessungsgrenze liegt. In diesem Lohnund Gehaltssegment dürften ältere Versicherte dominieren, für die ein Wechsel in die PKV kaum mehr in Frage kommt. Schließlich geht die Einschränkung der beitragsfreien Mitversicherung des Ehepartners nicht nur mit distributiven Normen konform, sondern vermindert über die dadurch mögliche Beitragssatzsenkung die Arbeitnehmer- sowie Arbeitgeberbeiträge und damit auch die Lohnnebenkosten. 


\section{e) Normierung, Fixierung und Auszahlung des Beitragssatzanteils des Arbeitgebers}

Beschäftigungs- und/oder wettbewerbspolitische Gründe könnten dafür sprechen, den Beitragssatzanteil des Arbeitgebers

- zu normieren,

- zu fixieren oder

- steuer- und beitragsneutral auszuzahlen.

Dabei können sich die Fixierung und die Auszahlung des Beitragssatzanteils des Arbeitgebers $u$. a. an dem (einen)

- geltenden kassenspezifischen Beitragssatz,

- durchschnittlichen Beitragssatz,

- Mindestbeitragssatz einer wählbaren Krankenkasse oder

- Beitragssatz zwischen dem durchschnittlichen und dem Mindestbeitragssatz

orientieren.

Mit Ausnahme des geltenden kassenspezifischen Beitragssatzes bieten sich diese Alternativen auch für den Fall einer Normierung des Beitragssatzanteils des Arbeitgebers an. Die Fixierung und die Auszahlung des Beitragssatzanteils des Arbeitgebers führen dazu, daß die Arbeitskosten der Unternehmen von künftigen Beitragssatzsteigerungen unberührt bleiben. Sofern sich die Fixierung und die Auszahlung an einem Beitragssatz orientieren, der unterhalb des durchschnittlichen liegt, kommt es für die Unternehmen insgesamt sogar unmittelbar zu einer Reduktion der Arbeitskosten. Dies setzt allerdings voraus, daß es den Arbeitnehmern bzw. Gewerkschaften nicht gelingt, diese Effekte im Rahmen der Tarifvereinbarungen zu kompensieren. Im Unterschied zur Fixierung und zur Auszahlung beteiligt die Normierung des Beitragssatzanteils des Arbeitgebers die Unternehmen weiterhin an Beitragssatzsteigerungen. Sie verstärkt jedoch ebenfalls über eine Spreizung der Beitragssatzanteile der Arbeitnehmer die Anreize, eine leistungsfähige Krankenkasse zu wählen, und besitzt insofern in erster Linie eine wettbewerbspolitische Funktion.

Die Fixierung und die Auszahlung des Arbeitgeber-Beitragssatzanteils koppeln die Entwicklung der Beitragssätze von den Arbeitskosten und damit auch von den privaten Investitionen ab. Für 
eine solche Trennung sprechen zunächst arbeitsmarktpolitische Überlegungen, denn die derzeitige Arbeitslosigkeit wurzelt primär in einer zu geringen Nachfrage nach Arbeitskräften. Der künftig stark ansteigende Anteil von Aufwendungen für Rentner an den gesamten Gesundheitsausgaben legt es ebenfalls nahe, die Investitionen nicht mit konsumtiven Ausgaben zu belasten. Ferner überantworten Fixierung und Auszahlung - in geringerem Umfang auch die Normierung - des Beitragssatzanteils des Arbeitgebers die Gestaltung der GKV ausschließlich den Arbeitnehmer und stärken damit deren Autonomie. Schließlich geht es im Rahmen der GKV um die Gesundheit der Versicherten, und die Mitwirkung der Arbeitgeber an der gemeinsamen Selbstverwaltung läßt sich aus dieser Perspektive als ein paternalistisches Relikt aus feudalen Zeiten interpretieren.

Die Kritiker einer Fixierung oder Auszahlung des ArbeitgeberBeitragssatzanteils sehen in der Aufgabe der paritätischen Finanzierung der Beitragssätze ein wesentliches Element des Sozialstaates in Gefahr. Zudem befürchten sie, daß der Abbau der paritätischen Finanzierung dann von der GKV auch auf die anderen Teilsysteme der sozialen Sicherung übergreift. Ferner erzeugt nach ihrer Meinung das Zusammenwirken von Arbeitgebern und Gewerkschaften im Rahmen der gemeinsamen Selbstverwaltung in politischer Hinsicht ein stabilisierendes Klima für die Tarifpolitik. Unbeschadet der rein politischen Aspekte gilt es hier in ökonomischer Hinsicht zu berücksichtigen, daß die Arbeitgeber ihren Beitragssatzanteil zwar finanzieren, aber keineswegs aus den $\mathrm{Ge}-$ winnen tragen, sondern wie andere Arbeitskosten über den Preis der entsprechenden Güter und Dienste auf die Konsumenten überwälzen. Unter bestimmten Bedingungen kann auch eine Rückwälzung auf die Arbeitnehmer in Form einer Lohnsenkung erfolgen. Hinsichtlich der Beitragsinzidenz stellt die paritätische Finanzierung weitgehend eine sozialpolitische Illusion dar. Pointiert formuliert trägt auch ein Beamter, wenn er Güter und Dienste am Markt erwirbt, über den Preis einen Teil der GKV-Beiträge.

\section{Modellvorhaben und Strukturverträge als Instrumente einer Wettbewerbsorientierung in der GKV}

Mit der Freigabe der Krankenkassenwahl für ca. $80 \%$ der GKVVersicherten stellte das GSG zwar die Weichen für eine Intensivierung des Wettbewerbs, wettbewerbliche Beziehungen zwischen den gesetzlichen Krankenkassen und auch zwischen die- 
sen und den privaten Krankenversicherungen existierten aber schon vorher. Dieser Wettbewerb vor dem GSG fand jedoch ohne eine konzeptionelle Rahmenordnung statt, und es fehlte insofern auch eine faire Ausgangsbasis für die konkurrierenden Krankenkassen. Die Beitragssätze, die sich zum 01.01.1994 in den alten Bundesländern zwischen $6,0 \%$ und $16,8 \%$ des Grundlohns bewegten, spiegelten nicht Unterschiede in der Leistungsfähigkeit der jeweiligen Krankenkassen wider, sondern wurzelten primär in einer institutionell verankerten Risikoselektion. Die Konzipierung einer wettbewerblichen Rahmenordnung für die GKV zielt $u$. a. darauf $a b$, diesen eingeschränkten Wettbewerbsprozeß, der willkürlich und asymmetrisch verlief, um notwendige Elemente zu erweitern und auf diese Weise in zielorientierte Bahnen zu lenken.

In konkreter Hinsicht strebt eine Wettbewerbsorientierung auf der Grundlage des Solidaritätsprinzips vor allem folgende Funktionen an:

- Schaffung von materieller Wahlfreiheit für jeden Versicherten,

- stärkere Orientierung der Gesundheitsversorgung an den Präferenzen der Versicherten und den Wünschen der Patienten,

- Erhöhung der Qualität der Gesundheitsversorgung,

- Verbesserung der Effizienz der Leistungserbringung,

- Setzen von Anreizen zu innovativen Suchprozessen, d. h. zu Produkt- und Prozeßinnovationen sowie neuen Versorgungsformen, und

- schnellere Anpassung an veränderte Rahmenbedingungen.

Zunächst bildet die Wahlfreiheit der Versicherten nicht nur ein Mittel zur Steigerung von Effizienz und Effektivität der Gesundheitsversorgung, sondern im Spannungsfeld von Autonomie und Abhängigkeit auch einen „Wert an sich“. Sodann sollten sich nach demokratischem und auch marktwirtschaftlichem Verständnis die Maßnahmen von Politikern und Unternehmen an den Wünschen der Bürger bzw. an den Präferenzen der Konsumenten orientieren. Dies bedeutet, daß in normativer Hinsicht die Präferenzen des Versicherten bzw. Patienten im Mittelpunkt der Gesundheitsversorgung stehen und nicht die Eigeninteressen von Leistungs- 
erbringern oder Krankenkassen. Der Versicherte bzw. Patient kann als Prinzipal an Souveränität gewinnen, wenn er über die Option der Kassenwahl verfügt und sich nicht in der Rolle des Bittstellers einer Einheitskasse oder einem monolithischen Kassenblock gegenübersieht. Eine Wahlfreiheit bei Gesundheitsleistungen und/oder Beitragstarifen vermag zwar auch eine Einheitskasse zu bieten, aber nur wenn die Versicherten die Option des Kassenwechsels besitzen, sehen sich die Krankenkassen gezwungen, ihr Versorgungsangebot stärker an den Wünschen und Bedürfnissen von Versicherten und Patienten auszurichten. Wahlmöglichkeiten erübrigen dann auch paternalistische Überlegungen über den Willen von Versicherten und Patienten.

Eine Wahlmöglichkeit für die Nachfrager bietet zwar auch ein privates Krankenversicherungssystem, die systemhafte Neuerung einer wettbewerblichen Orientierung der GKV besteht jedoch darin, daß der Wettbewerb hier bei Beachtung des Solidaritätsprinzips abläuft. Letzteres stellt an Ausgestaltung und Implementierung des Wettbewerbsprinzips vor allem folgende Anforderungen:

(1) Es gilt für alle Kassen unabhängig vom jeweiligen Krankheitsrisiko des Nachfragers bzw. potentiellen Versicherten grundsätzlich Kontrahierungszwang und Diskriminierungsverbot.

(2) Alle bisherigen Umverteilungselemente, die das Solidaritätsprinzip in der GKV prägen bzw. charakterisieren, bleiben erhalten (siehe oben unter 2).

(3) Unabhängig von den verschiedenen Versicherten- und Risikostrukturen, die beim Startschuß zum Wettbewerb vorherrschen, muß jede Kasse eine faire Wettbewerbschance erhalten.

(4) Das System darf keine Anreize setzen, durch Risikoselektion Wettbewerbsvorteile zu erlangen.

Die Integration des Wettbewerbsprinzips in ein System solidarischer Krankenversicherung zielt somit darauf ab, über erweiterte Wahlmöglichkeiten der Versicherten bei Krankenkassen und Leistungserbringern stärkere Anreize zur Erhöhung von Effizienz und Effektivität der Gesundheitsversorgung zu setzen, d. h. einen entsprechenden Wettbewerbsdruck zu erzeugen. Insofern bildet die Zahl der Kassenwechsler weder ein Funktionsziel der Wettbewerbsorientierung noch zwangsläufig einen validen Indikator 
der Wettbewerbsintensität. Für die Realisierung der wettbewerblichen Funktion reicht es völlig aus, wenn die Krankenkassen mit dem $\mathrm{Ab}$ - und Zuwandern von Versicherten rechnen müssen und sich deshalb um eine effiziente und effektive Gesundheitsversorgung bemühen. Da der durch das GSG induzierte Kassenwettbewerb auf der Grundlage aller bisherigen Elemente des Solidarprinzips abläuft, vermeidet er schon vom konzeptionellen Ansatz her jene Ausgrenzungen und negativen Verteilungswirkungen, die z. B. wettbewerblichen Managed-Care-Konzepten auf dem gänzlich anders gearteten amerikanischen Krankenversicherungsmarkt anhaften. Die Kombination von Solidarität und Wettbewerb strebt nach einem dritten bzw. Mittelweg zwischen völliger Marktöffnung mit durchgehend risikoäquivalenten Prämien und einem weitgehend verstaatlichten Krankenversicherungssystem.

Das Wahlrecht der Versicherten kann nur dann eine funktionsgerechte Intensivierung des Wettbewerbs zwischen den Krankenkassen auslösen, wenn zumindest partiell eine Flexibilisierung des Vertragsrechtes hinzutritt. Sofern die Krankenkassen einheitlich und gemeinsam mit den Leistungserbringern in vertragliche Beziehungen treten sowie Umfang, Art und Qualität der Versorgung einheitlich festlegen, besitzt der Versicherte nur ein formales, aber kein materielles Wahlrecht. Da der RSA die Beitragssätze der einzelnen Krankenkassen unter diesen uniformen Bedingungen weitgehend annähert, existiert für den Versicherten zwischen einer solchen Kassenlandschaft und einer Einheitskasse kein nennenswerter Unterschied mehr. Der Wettbewerb der Krankenkassen um Versicherte zieht im Sinne einer effizienten und effektiven Gesundheitsversorgung als logische Konsequenz wettbewerbliche Strukturen im Bereich zwischen Krankenkassen und Leistungserbringern nach sich.

Die Flexibilisierung des Vertragsrechts reicht alleine noch nicht aus, um eine effizientere und effektivere Gesundheitsversorgung zu verwirklichen. Die Krankenkassen benötigen auch auf der Leistungsseite Wettbewerbsparameter, um sich gegenüber den Versicherten und Patienten in einer spezifischen Weise zu profilieren. Begrenzte Wahlmöglichkeiten können sowohl hinsichtlich der Versorgungsform (z. B. Hausarztmodelle oder kombinierte Budgets als Alternative) als auch beim Leistungskatalog (z. B. bei Kuren, beim Zahnersatz, bei alternativen Heilmethoden und bei Segmenten von nichtverschreibungspflichtigen Arzneimitteln) dazu beitragen, das Leistungsangebot besser auf die Präferenzen 
der Versicherten abzustimmen. Sofern die Krankenkassen über Wettbewerbsparameter auf der Leistungsseite verfügen, besteht auch für die Leistungserbringer ein Anreiz, nach Problemlösungen zu suchen, die sich an den speziellen Wünschen der Krankenkassen orientieren.

Die Modellvorhaben und Strukturverträge nach $\S \S 63 \mathrm{ff}$. und 73 a SGB $V$ bilden im wesentlichen die gesetzlichen Grundlagen für die speziellen Gestaltungsspielräume der Krankenkassen. Unter den gegebenen gesundheitspolitischen Rahmenbedingungen hilft es wenig, im Hinblick auf die dritte Stufe der Gesundheitsreform einem erheblich weiteren Spektrum an Wettbewerbsparametern nachzutrauern, vielmehr gilt es, die gegebenen Möglichkeiten optimal zu nutzen. Den Modellvorhaben und Strukturverträgen fällt somit die Aufgabe zu, die Gestaltungsspielräume auszuloten, die der Gesetzgeber für neue innovative Versorgungsformen eröffnete. Bleiben diese unausgeschöpft, stagniert nicht nur die Wettbewerbsorientierung in der GKV, es droht darüber hinaus in der deutschen Gesundheitsversorgung ein struktureller Stillstand auf absehbare Zeit. Im Rahmen von Modellvorhaben und Strukturverträgen könnte der Auf- und Ausbau von Managed-CareKonzepten darüber hinaus die Notwendigkeit weiterer Flexibilisierungen im deutschen Gesundheitswesen transparent machen und möglicherweise entsprechende Prozesse stimulieren bzw. nach sich ziehen.

Die stärkere Wettbewerbsorientierung und in ihrem Kontext Modellvorhaben und Strukturverträge dienen nicht zuletzt dazu, dezentrale innovative Suchprozesse nach effizienteren und effektiveren Versorgungsstrukturen auszulösen. Führen solche Neuerungen nicht zu dem gewünschten Erfolg, beschränken sich ihre Auswirkungen im Gegensatz zur Regelversorgung auf einen abgegrenzten Kreis von Leistungserbringern und Patienten, die freiwillig an diesem Versorgungsmodell teilnehmen. Im Erfolgsfall dagegen können innovative Managed-Care-Konzepte im Zuge der Nachahmung bzw. Diffusion in die Regelversorgung übergehen und so über den Kreis der unmittelbar bzw. zunächst Beteiligten hinaus quasi positive externe Effekte für die gesamte Bevölkerung auslösen. 


\section{Literatur}

Arnold, Michael (1995), Solidarität 2000. Die medizinische Versorgung und ihre Finanzierung nach der Jahrtausendwende, 2. Aufl., Stuttgart.

Arnold, Michael, Lauterbach, Karl W. und Preuß, Klaus-Jürgen, Hrsg. (1997), Managed Care. Ursachen, Prinzipien, Formen und Effekte, Stuttgart, New York.

Behrens, Johann et al., Hrsg. (1996), Gesundheitssystementwicklung in den USA und Deutschland. Wettbewerb und Markt als Ordnungselemente im Gesundheitswesen auf dem Prüfstand des Systemvergleichs, Baden-Baden.

Berié, Hermann und Fink, Ulf (1997), Wo liegen die finanziellen Probleme der gesetzlichen Krankenversicherung? Institut für Wirtschaft und Soziales, Berlin, August 1997.

Breyer, Friedrich (1997), „Beitragsfreie Mitversicherung“ und „Familienlastenausgleich" in der GKV: ein populärer Irrtum, in: Konjunkturpolitik, 43. Jg., Heft 3, S. 213-223.

Busch, Susanne, Pfaff, Anita B. und Rindsfüßer, Christian (1996), Die Finanzierung der gesetzlichen Krankenversicherung. Möglichkeiten zur Umgestaltung und Ergebnisse ausgewählter Modellrechnungen, Düsseldorf.

Deutsche Bundesbank (1997), Die fiskalische Belastung zukünftiger Generationen - eine Analyse mit Hilfe des General Accounting, in: Monatsberichte der Deutschen Bundesbank, 49. Jg., Nr. 11, S. 17-30.

Enquete-Kommission „Strukturreform der gesetzlichen Krankenversicherung“ (1990), Endbericht, in: Bundestagsdrucksache 11/6380 vom 12.02.1990, S. 152-164.

Erdmann, Yvonne (1995), Managed Care. Veränderungen im Gesundheitswesen der USA in den letzten 30 Jahren, Baden-Baden.

Münnich, Frank E. (1998), Veraltetes Strukturprinzip. Die lohnbezogene GKV-Finanzierung wird den neuen Herausforderungen nicht gerecht, in: Forum für Gesellschaftspolitik, Bonn, Januar 1998, S. 14-18

Sachverständigenrat für die Konzertierte Aktion im Gesundheitswesen (1994), Sachstandsbericht 1994. Gesundheitsversorgung und Krankenversicherung 2000, Baden-Baden.

Sachverständigenrat für die Konzertierte Aktion im Gesundheitswesen (1996), Sondergutachten 1996. Gesundheitswesen in Deutschland, Kostenfaktor und Zukunftsbranche, Bd. I: Demographie, Morbidität, Wirtschaftlichkeitsreserven und Beschäftigung, Baden-Baden.

Sachverständigenrat für die Konzertierte Aktion im Gesundheitswesen (1997), Sondergutachten 1997. Gesundheitswesen in Deutschland, Kostenfaktor und Zukunftsbranche, Bd. II: Fortschritt und Wachstumsmärkte, Finanzierung und Vergütung, Baden-Baden.

Toepffer, Johannes (1997), Krankenversicherung im Spannungsfeld von Markt und Staat. Das Beispiel USA und seine Implikationen für Funktion und Gestaltung eines marktwirtschaftlich orientierten Krankenversicherungssystems, Bayreuth. 
Albring, Dr. med. Manfred

Arnold, Prof. Dr. med. Dr. hc.

Michael

Bausch, Dr. med. Jürgen

Brech, Prof. Dr. med. Wolfgang

Bürger, Dr. oec. Babette

Cassel, Prof. Dr. rer. pol. Dieter

Dierks, Dr. med. Dr. jur Christian

Ehlers, Dr. med. Dr. jur. Alexander P. F.

Firnkorn, Hans-Jürgen

Gitter, Prof. Dr. jur. Wolfgang

Glaeske, Dr. rer. nat. Gerd

Granitza, Dr. jur. Axel

Hoberg, Dr. rer. pol. Rolf
Leiter Medizin/Gesundheitswesen

Schering Aktiengesellschaft Geschäftsbereich Deutschland, Berlin

Wissenschaftspolitische Initiativen des Stifterverbandes e. V., Tübingen

Vorsitzender der Kassenärztlichen Vereinigung Hessen, Frankfurt

Vorsitzender der Kassenärztlichen Vereinigung Südwürttemberg, Reutlingen

Referentin des Vorsitzenden des gesundheitsökonomischen Ausschusses der FDP Dr. Thomae (MdB), Bonn

Lehrstuhl für Wirtschaftspolitik an der Universität Duisburg

Dierks \& Bohle, Rechtsanwälte, Berlin

Rechtsanwaltssocietät Ehlers, Ehlers \& Partner, München

Leiter Referat und Gesundheitspflege der Robert-Bosch-Stiftung $\mathrm{GmbH}$, Stuttgart

Lehrstuhl für Zivilrecht II, RW, an der Universität Bayreuth

Leiter Abteilung Medizinisch-Wissenschaftliche Grundsatzfragen, BARMER Ersatzkasse Hauptverwaltung, Wuppertal

Bereichsleiter Konzernstäbe, Schering Aktiengesellschaft, Berlin

Mitglied des Vorstandes der AOK Baden-Württemberg, Stuttgart 
Klemm, Dr. med. Helmut

Klusen, Dr. oec. Norbert

Knieps, Franz

Krimmel, Dr. med. Lothar

Laschet, Helmut

Lohmann, Wolfgang

Münnich, Prof. Dr. rer. pol. Frank

Pfaff, Prof. Dr. rer. pol. Anita

Reischl, Wilfried

Richter-Reichhelm, Dr. med. Manfred

Schirmer, Herwig F.

Schmeinck, Wolfgang

Schulte, Gerhard stellvertretender Vorsitzender der Kassenärztlichen Vereinigung Bayern, Bezirksstelle Oberbayern, München; Vorsitzender der Vertreterversammlung der Kassenärztlichen Bundesvereinigung, Köln

Vorstandsvorsitzender der Techniker Krankenkasse, Hamburg

Leiter der Abteilung Verbandspolitische Planung, AOK Bundesverband, Bonn

stellvertretender Hauptgeschäftsführer der Kassenärztlichen Bundesvereinigung, Köln

stellvertretender Chefredakteur der Ärztezeitung, Neu-Isenburg

Mitglied des Deutschen Bundestages, Bonn

Königswinter

Institut für Volkswirtschaftslehre an der Universität Augsburg und Internationales Institut für Sozialökonomie, Leitershof-Stadtbergen

Referent der Arbeitsgruppe Gesundheit der CDU/CSU, Bonn

Vorsitzender der Kassenärztlichen Vereinigung Berlin,

Staatssekretär im Ministerium für Arbeit, Soziales, Gesundheit und Frauen, Potsdam

Vorsitzender des Vorstandes des Bundesverbandes der Betriebskrankenkassen, Essen

Vorstandsvorsitzender des Landesverbandes der Betriebskrankenkassen in Bayern, München 
Schwoerer, Dr. med. Peter

Smigielski, Dr. rer. oec. Edwin

Stillfried, Dr. rer. pol. Dominik Graf von

Thomae, Dr. rer. pol. Dieter

Uleer, Dr. jur. Christoph

Walzik, Eva

Weller, Michael

Wille, Prof. Dr. rer. pol. Eberhard

Zipperer, Dr. jur. Manfred
Beratender Arzt des Medizinischen Dienstes Baden-Württemberg, Lahr

Geschäftsführer der Abteilung Politik beim Verband der Forschenden Arzneimittelhersteller e. V., Bonn

Abteilung Medizinisch-Wissenschaftliche Grundsatzfragen bei der BARMER Ersatzkasse, Wuppertal

Mitglied des Deutschen Bundestages und Vorsitzender des gesundheitsökonomischen Ausschusses der FDP, Bonn

Verbandsdirektor des Verbandes der privaten Krankenversicherung e. V., Köln

stellvertretende Leiterin der Abteilung Verbandspolitik - Marktsicherung - Öffentlichkeitsarbeit, Verband der Angestellten-Krankenkassen e. V., Siegburg

Referent der Arbeitsgruppe Gesundheit der SPD, Bonn

Lehrstuhl für Volkswirtschaftslehre an der Universität Mannheim

Ministerialdirektor, Abteilungsleiter der Abteilung II Gesundheitsversorgung, Krankenversicherung im Bundesministerium für Gesundheit, Bonn 


\section{STAATLICHE ALLOKATIONSPOLITIK IM MARKTWIRTSCHAFTLICHEN SYSTEM}

Band

Band

Band

Band

Band

Band

Band

Band

Band

1 Horst Siebert (Hrsg.): Umweltallokation im Raum. 1982.

2 Horst Siebert (Hrsg.): Global Environmental Resources. The Ozone Problem. 1982.

3 Hans-Joachim Schulz: Steuerwirkungen in einem dynamischen Unternehmensmodell. Ein Beitrag zur Dynamisierung der Steuerüberwälzungsanalyse. 1981.

4 Eberhard Wille (Hrsg.): Beiträge zur gesamtwirtschaftlichen Allokation. Allokationsprobleme im intermediären Bereich zwischen öffentlichem und privatem Wirtschaftssektor. 1983.

5 Heinz König (Hrsg.): Ausbildung und Arbeitsmarkt. 1983.

6 Horst Siebert (Hrsg.): Reaktionen auf Energiepreissteigerungen. 1982.

7 Eberhard Wille (Hrsg.): Konzeptionelle Probleme öffentlicher Planung. 1983.

8 Ingeborg Kiesewetter-Wrana: Exporterlösinstabilität. Kritische Analyse eines entwicklungspolitischen Problems. 1982.

9 Ferdinand Dudenhöfer: Mehrheitswahl-Entscheidungen über Umweltnutzungen. Eine Untersuchung von Gleichgewichtszuständen in einem mikroökonomischen Markt- und Abstimmungsmodell. 1983.

Band 10 Horst Siebert (Hrsg.): Intertemporale Allokation. 1984.

Band 11 Helmut Meder: Die intertemporale Allokation erschöpfbarer Naturressourcen bei fehlenden Zukunftsmärkten und institutionalisierten Marktsubstituten. 1984.

Band 12 Ulrich Ring: Öffentliche Planungsziele und staatliche Budgets. Zur Erfüllung öffentlicher Aufgaben durch nicht-staatliche Entscheidungseinheiten. 1985.

Band 13 Ehrentraud Graw: Informationseffizienz von Terminkontraktmärkten für Währungen. Eine empirische Untersuchung. 1984.

Band 14 Rüdiger Pethig (Ed.): Public Goods and Public Allocation Policy. 1985.

Band 15 Eberhard Wille (Hrsg.): Öffentliche Planung auf Landesebene. Eine Analyse von Planungskonzepten in Deutschland, Österreich und der Schweiz. 1986.

Band 16 Helga Gebauer: Regionale Umweltnutzungen in der Zeit. Eine intertemporale Zwei-Regionen-Analyse. 1985.

Band 17 Christine Pfitzer: Integrierte Entwicklungsplanung als Allokationsinstrument auf Landesebene. Eine Analyse der öffentlichen Planung der Länder Hessen, Bayern und Niedersachsen. 1985.

Band 18 Heinz König (Hrsg.): Kontrolltheoretische Ansätze in makroökonometrischen Modellen. 1985.

Band 19 Theo Kempf: Theorie und Empirie betrieblicher Ausbildungsplatzangebote. 1985.

Band 20 Eberhard Wille (Hrsg.): Konkrete Probleme öffentlicher Planung. Grundlegende Aspekte der Zielbildung, Effizienz und Kontrolle. 1986.

Band 21 Eberhard Wille (Hrsg.): Informations- und Planungsprobleme in öffentlichen Aufgabenbereichen. Aspekte der Zielbildung und Outputmessung unter besonderer Berücksichtigung des Gesundheitswesens. 1986.

Band 22 Bernd Gutting: Der Einfluß der Besteuerung auf die Entwicklung der Wohnungs- und Baulandmärkte. Eine intertemporale Analyse der bundesdeutschen Steuergesetze. 1986.

Band 23 Heiner Kuhl: Umweltressourcen als Gegenstand internationaler Verhandlungen. Eine theoretische Transaktionskostenanalyse. 1987. 
Band 24 Hubert Hombach: Besteuerung, Inflation und Kapitalallokation. Intersektorale und internationale Aspekte. 1987.

Band 25 Peter Müller: Intertemporale Wirkungen der Staatsverschuldung. 1987.

Band 26 Stefan Kronenberger: Die Investitionen im Rahmen der Staatsausgaben. 1988.

Band 27 Armin-Detlef Rieß: Optimale Auslandsverschuldung bei potentiellen Schuldendienstproblemen. 1988.

Band 28 Volker Ulrich: Preis- und Mengeneffekte im Gesundheitswesen. Eine Ausgabenanalyse von GKV-Behandlungsarten. 1988.

Band 29 Hans-Michael Geiger: Informational Efficiency in Speculative Markets. A Theoretical Investigation. Edited by Ehrentraud Graw. 1989.

Band 30 Karl Sputek: Zielgerichtete Ressourcenallokation. Ein Modellentwurf zur Effektivitätsanalyse praktischer Budgetplanung am Beispiel von Berlin (West). 1989.

\section{ALLOKATION IM MARKTWIRTSCHAFTLICHEN SYSTEM}

Band 31 Wolfgang Krader: Neuere Entwicklungen linearer latenter Kovarianzstrukturmodelle mit quantitativen und qualitativen Indikatorvariablen. Theorie und Anwendung auf ein mikroempirisches Modell des Preis-, Produktions- und Lageranpassungsverhaltens von deutschen und französischen Unternehmen des verarbeitenden Gewerbes. 1991.

Band 32 Manfred Erbsland: Die öffentlichen Personalausgaben. Eine empirische Analyse für die Bundesrepublik Deutschland. 1991.

Band 33 Walter Ried: Information und Nutzen der medizinischen Diagnostik. 1992.

Band 34 Anselm U. Römer: Was ist den Bürgern die Verminderung eines Risikos wert? Eine Anwendung des kontingenten Bewertungsansatzes auf das Giftmüllrisiko. 1993.

Band 35 Eberhard Wille, Angelika Mehnert, Jan Philipp Rohweder: Zum gesellschaftlichen Nutzen pharmazeutischer Innovationen. 1994.

Band 36 Peter Schmidt: Die Wahl des Rentenalters. Theoretische und empirische Analyse des Rentenzugangsverhaltens in West- und Ostdeutschland. 1995.

Band 37 Michael Ohmer: Die Grundlagen der Einkommensteuer. Gerechtigkeit und Effizienz. 1997.

Band 38 Evamaria Wagner: Risikomanagement rohstoffexportierender Entwicklungsländer. 1997.

Band 39 Matthias Meier: Das Sparverhalten der privaten Haushalte und der demographische Wandel: Makroökonomische Auswirkungen. Eine Simulation verschiedener Reformen der Rentenversicherung. 1997.

Band 40 Manfred Albring / Eberhard Wille (Hrsg.): Innovationen in der Arzneimitteltherapie. Definition, medizinische Umsetzung und Finanzierung. Bad Orber Gespräche über kontroverse Themen im Gesundheitswesen 25.-27.10.1996. 1997.

Band 41 Eberhard Wille / Manfred Albring (Hrsg.): Reformoptionen im Gesundheitswesen. Bad Orber Gespräche über kontroverse Themen im Gesundheitswesen 7.-8.11.1997. 1998. 